\title{
Nederlanders and buitenlanders
}

\author{
Citation for published version (APA):
}

van de Weerd, L. P. (2020). Nederlanders and buitenlanders: A sociolinguistic-ethnographic study of ethnic categorization among secondary school pupils. [Doctoral Thesis, Maastricht University, Université Libre de Bruxelles]. LOT. https://doi.org/10.26481/dis.20201118lp

\section{Document status and date:}

Published: 01/01/2020

DOI:

10.26481/dis.20201118lp

Document Version:

Publisher's PDF, also known as Version of record

\section{Please check the document version of this publication:}

- A submitted manuscript is the version of the article upon submission and before peer-review. There can be important differences between the submitted version and the official published version of record.

People interested in the research are advised to contact the author for the final version of the publication, or visit the DOI to the publisher's website.

- The final author version and the galley proof are versions of the publication after peer review.

- The final published version features the final layout of the paper including the volume, issue and page numbers.

Link to publication

\footnotetext{
General rights rights.

- You may freely distribute the URL identifying the publication in the public portal. please follow below link for the End User Agreement:

www.umlib.nl/taverne-license

Take down policy

If you believe that this document breaches copyright please contact us at:

repository@maastrichtuniversity.nl

providing details and we will investigate your claim.
}

Copyright and moral rights for the publications made accessible in the public portal are retained by the authors and/or other copyright owners and it is a condition of accessing publications that users recognise and abide by the legal requirements associated with these

- Users may download and print one copy of any publication from the public portal for the purpose of private study or research.

- You may not further distribute the material or use it for any profit-making activity or commercial gain

If the publication is distributed under the terms of Article $25 \mathrm{fa}$ of the Dutch Copyright Act, indicated by the "Taverne" license above, 


\title{
Nederlanders and buitenlanders:
}

\author{
A sociolinguistic-ethnographic study of ethnic \\ categorization among secondary school pupils
}


Published by

LOT

Kloveniersburgwal 48

phone: +31205252461

1012 CX Amsterdam

The Netherlands

e-mail: lot@uva.nl

http://www.lotschool.nl

Cover illustration: Kelly Breemer

ISBN: 978-94-6093-365-3

NUR: 616

Copyright (C) 2020: Pomme van de Weerd. All rights reserved. 


\section{Nederlanders and buitenlanders:}

A sociolinguistic-ethnographic study of ethnic categorization among secondary school pupils

\section{DISSERTATION}

to obtain the degree of Doctor at the Maastricht University, and the degree of Docteur en Langues, Lettres et Traductologie at the Université Libre de Bruxelles, on the authority of the Rector Magnificus of Maastricht University and the Rector of the Universite libre de Bruxelles,

Prof. dr. Rianne M. Letschert and Prof. dr. Annemie Schaus in accordance with the decision of the Board of Deans, to be defended in public on Wednesday 18 November 2020, at 16:00 hours

by

Lisa Pomme van de Weerd 


\title{
Supervisors
}

Prof. dr. L. Cornips (Maastricht University and KNAW)

Prof. dr. J. Jaspers (Université Libre de Bruxelles)

\section{Co-supervisor}

Prof. dr. E. Wesseling (Maastricht University)

\author{
Assessment committee \\ Prof. dr. R. van de Vall (Chair) (Maastricht University) \\ Dr. P. De Brabanter (Université Libre de Bruxelles) \\ Prof. dr. M. Bucholtz (University of California, Santa Barbara) \\ Dr. L. Calabrese (Université Libre de Bruxelles) \\ Dr. L. Malai Madsen (University of Copenhagen) \\ Prof. dr. V. Mazzucato (Maastricht University)
}




\section{Contents}

Acknowledgements ....................................................... 7

1 Introduction............................................................. 9

1.1 Theoretical and methodological foundations............................... 13

1.2 Roadmap of the dissertation ......................................................... 33

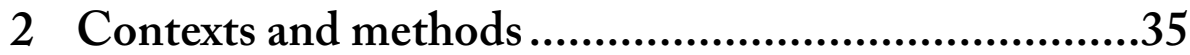

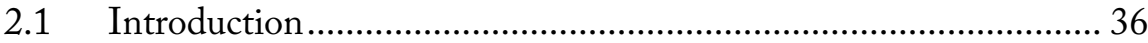

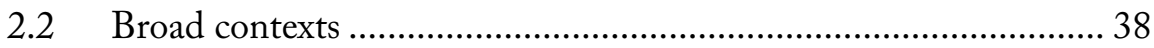

$2.3 \quad$ Fieldwork context and methods................................................. 49

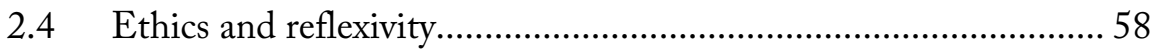

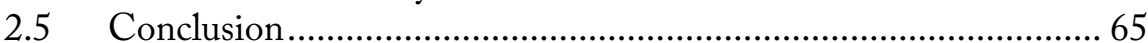

3 Negotiating the characteristics of Turken, Marokkanen, buitenlanders, and Nederlanders .........67

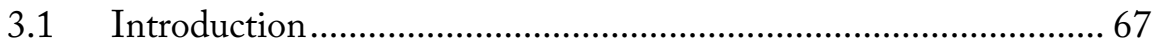

3.2 The meaning potential of ethnic categories .................................. 69

3.3 Marokkanen, Turken, buitenlanders, and Nederlanders and debates about locally associated characteristics ........................................... 70

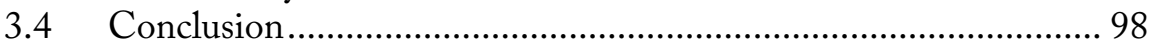

4 Interactional functions of ethnic labels .....................101

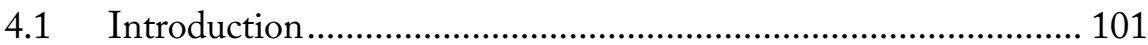

4.2 Categories and action in interaction ......................................... 103

4.3 Ethnic labels and power positions in interaction ........................ 104

4.4 Ethnic labels and jocular mockery ............................................. 113

4.5 Mockery by a non-member of the mocked category................... 121

4.6 Conclusion............................................................................... 132

5 Categorization in interactions involving teachers .....135

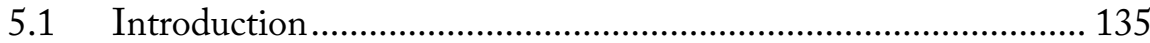

5.2 Teacher and pupil categorizations in previous literature............. 136

5.3 Teachers' problematizations of categories.................................. 137 


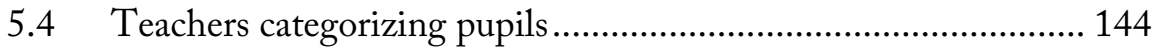

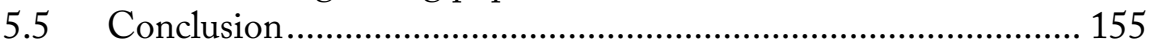

6 The use of linguistic resources in relation to categorization practices .........................................157

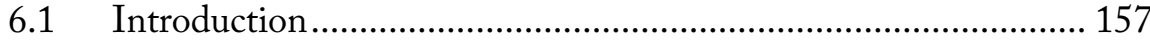

6.2 Context: Languages and their positions in the Netherlands....... 160

6.3 Language use and ideologies in class 3/4b .................................. 164

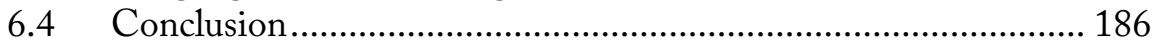

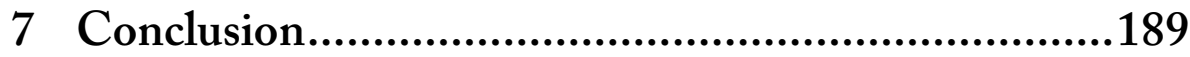

7.1 Summary of main findings...................................................... 190

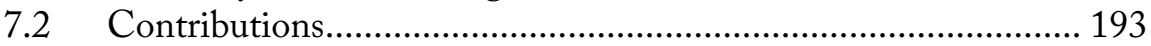

7.3 Representativity and suggestions for further research ................ 198

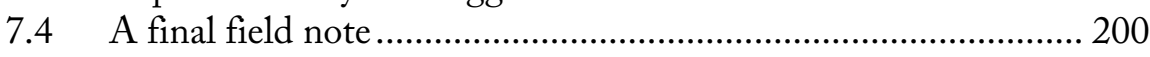

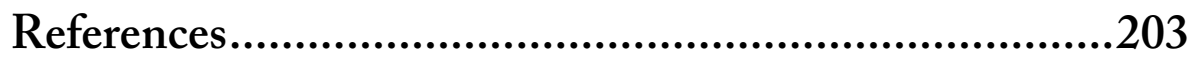

Nederlandse samenvatting........................................225

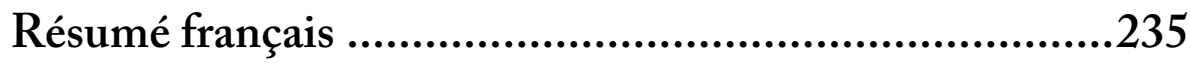

Valorization addendum ............................................245

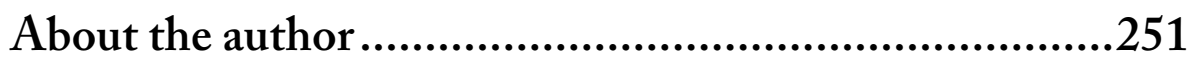




\section{Acknowledgements}

Bringing this $\mathrm{PhD}$ to a successful end would not have been possible by myself. Here, I would like to take the opportunity to say a word of thanks to the people that have been there for me along the way.

First of all, my supervisors. Leonie, I have felt supported by you at all times and in many different ways during these four years. You have attention for the practical and academic aspects of doing a $\mathrm{PhD}$, but your keen eye for the emotional and psychological aspects stands out even more. This make you a wonderful supervisor, and if I ever become a supervisor too, I hope to take after you. Jürgen, you have the gift of reading a draft and looking right into my head, to then formulate the idea I was trying to describe in much more accurate words. On top of that, and at least as often, your critical (though forever constructive) feedback have brought my analyses to a next level. I am very happy and thankful that we turned this into a joint doctorate and that you became my second promotor. Lies, with your office next to mine, I always felt welcome to come by with any questions. Thank you for providing me with the views of someone outside of my discipline and reminding me to speak to a broader audience.

Of course, I am indebted to my research participants, whose names I cannot use here. Aan de leerlingen en docenten van wat ik in dit proefschrift 'klas 3/4b' van 'South High School' heb genoemd: bedankt dat ik jullie mocht volgen door de school, dat ik jullie mocht opnemen, en onwetende vragen mocht stellen, en dat alles negen maanden lang. Ik heb me door jullie geaccepteerd en opgenomen gevoeld, en heb veel geleerd door tijd met jullie te besteden. We hebben veel gelachen samen, en nog steeds vertel ik graag anekdotes waarin jullie de ster zijn. Ik hoop dat jullie dezelfde warme herinneringen bewaren als ik, en dat we elkaar ooit weer mogen ontmoeten. Bedankt dat ik met, en van, jullie mocht leren.

Doing a $\mathrm{PhD}$ can be a lonely experience, but thanks to the ever-growing Languageculture team (Lotte, Jolien, Lysbeth, Nantke, Daan, Ilias, Marie, Sara) I have felt embedded in a social structure. Our meetings have always been intellectually stimulating, but also socially engaging and fun, making the hard work that much more enjoyable. I feel proud to be part of this great and unique team of researchers. Thanks to my office roommates Nantke, Daan, en Veerle, and at the very end, Iskander, for creating the perfect balance between an open atmosphere and chats, to shared concentration and hard work. Thanks to the members of the Transnational Migration Group for 


\section{Nederlanders and buitenlanders}

providing feedback on papers and presentations and setting up and engaging in valuable discussions.

During my PhD I spent a few months on a research visit at University of California, Santa Barbara. I would like to thank Mary Bucholtz for receiving me, discussing my work with me, and putting me in contact with some of the amazing people working alongside her. Thank you also to Kevin Whitehead for inviting me to participate in his course in Conversation Analysis and providing me with valuable feedback on some parts of this dissertation, and to the folks in the graduate sessions for the data sessions.

Thank you to my paranymphs! Daan, we started this journey at about the same time, thanks for supporting me across the finish line. Vicky, how lucky that you turned out to be in Maastricht at the same time as me, thank you for the dinners, drinks, and laughs.

Of course, the non-academic support that I have been lucky to have has been at least as valuable. Pap, mam, Roos, en Lein, bedankt voor jullie oneindige steun, die soms vanzelfsprekend voelt maar dat niet is, en voor het vieren mijn successen, zelfs als ik die soms zelf niet als zodanig wist te herkennen. Felix, dat ik het eind van mijn $\mathrm{PhD}$ zo goed ben doorgekomen is voor een groot deel aan jou te danken. Bedankt voor je vertrouwen en liefde. Kelly, wat een geluk dat je, naast een geweldige vriendin, ook een talentvolle illustrator en designer bent - bedankt voor het vormgeven van de prachtige kaft van dit proefschrift. Veel dank aan mijn vrienden en vriendinnen, die, door tijd met mij te besteden, me motiveerden om elke keer met nieuwe moed verder werken aan dit project. Ook dankzij jullie ligt dit boek hier. Ik noem jullie hier niet allen bij naam, maar weet dat ik dankbaar ben voor jullie vriendschap. Thank you to my friends: by spending time together, putting things into perspective, and enabling me to think about other things, you have helped me write this book. I am grateful for your friendship. 


\section{Introduction}

Math class, 3 April 2017

Amine: $\quad$ Eén minuut

$\begin{array}{ll}\text { One more minute } \\ \text { Teacher: } & \text { Amine, ik zeg toch nee, en dan ga je het toch doen }\end{array}$

Amine, I just said 'no,' and still you go on and do it

Mohammed: Nee is nee!

No means no!

Amira: $\quad$ Bij een tatta nee is nee, niet bij een Marokkaan

For a Dutch person no means no, not for a Moroccan

Care and Well-being class, 12 December 2017

Hatice: $\quad$ Ik vind Nederlandse kinderen zo raar hè

I find Dutch children so weird

Pomme: Wie vind je raar?

Who do you find weird?

Hatice: $\quad$ Nederlandse kinderen, zulke rare mensen

Dutch children, such weird people

Pomme: Haha, wat dan?

Haha, what about them?

Dounia: Nederlanders zijn allemaal raar, niet alleen de kinderen

Dutch people are all weird, not just the children

Hatice: Ja zijn allemaal raar, wollah

Yes they're all weird, wollah

Societal studies, 12 January 2017

Meryem: Het is zo heet hier

It is so hot here

Teacher: Waarom doe je je trui niet gewoon uit? Why don't you just take off your sweater?

(Meryem attempts to pull her sweater over her head but gets stuck with her headphones)

Amira: $\quad$ Eerst je oordopjes uit! Echt weer zo'n Turkse actie!

First remove your headphones! What a Turkish thing to do! 


\section{Nederlanders and buitenlanders}

Focusing on exchanges such as these, this dissertation studies patterns of social and linguistic stratification as enacted and constructed in interaction among secondary school pupils. It builds on ethnographic fieldwork at South High School (a pseudonym) in Venlo, the Netherlands, to show how, in ordinary conversations and through off-hand references, pupils such as Amira and Hatice categorized what they perceived as different kinds of people - as in the first two vignettes, 'Dutch' and 'Moroccan' people, or in the third vignette, 'Turkish' people. It will furthermore analyze how pupils negotiated local social hierarchies of these categories by associating them with certain characteristics, for instance as Hatice does by associating 'weirdness' with Nederlanders ('Dutch people'), and Amira by associating being 'Turkish' with clumsiness. By closely analyzing interactions on the local level such as those in the opening vignettes, the aim of the dissertation is to analyze how pupils negotiated local as well as wider societal social hierarchies through ethnic categorization and the use of different linguistic resources.

As illustrated in the opening interactions, the pupils in this study categorized themselves and others in what can be called ethnic terms, referring to themselves and others as buitenlanders 'foreigners,' Marokkanen 'Moroccans,' Turken 'Turks,' or Nederlanders 'Dutch people. ${ }^{1}$ In the Dutch context, such categorizations often invite public and political debates surrounding immigrant integration (i.e. 'Are people who refer to themselves as 'Moroccan' well-integrated into 'Dutch culture' and customs?') and the meaning of citizenship (i.e. 'What does it mean that some people do not consider themselves 'Dutch'?').

There is a pervasive notion that immigrants and their descendants can only orient to one 'culture' at the same time - 'the Moroccan culture,' 'the Turkish culture,' or 'the Dutch culture' (Slootman 2016) - or that they are caught in-between cultures (Ketner, Buitelaar, and Bosma 2004). The use of category labels for self-description that refer to one country or 'culture' is regularly understood to reveal such sociocultural orientations. If, for example. someone refers to themselves as Turk, it is often thought that this person (i) has a strong orientation to 'the Turkish culture' and (ii) therefore cannot feel 'at home' in, or 'belonging to' the Netherlands.

This perception is pervasive in popular discourse, but it is also reflected in some academic research that aims to measure people's feelings of 'belonging' to (or their 'sense of citizenship,' 'integration,' or 'identification with') the Netherlands and operationalizes this question by asking people to

\footnotetext{
${ }^{1}$ I translate the Dutch noun Nederlander/s as "Dutch" or as "Dutch person/people" instead of the more common, but gendered English translation "Dutchman/ Dutchmen."
} 
categorize themselves. This way, Dutch people with a Moroccan migration background have been said to 'feel more Moroccan than Dutch' (Azghari, Hooghiemstra, and van de Vijver 2015). Similarly, a majority of TurkishDutch participants of a study by Verkuyten and Yildiz $(2007,1459)$ responded to the question whether they 'feel Dutch' or 'identify with the Dutch' and were concluded to have 'low or neutral Dutch identification [and] neutral or high disidentification' (Verkuyten and Yildiz 2007, 1459). A study in Belgium, with its similar history of migration, stated that 84 percent of the 'Turkish minority group' and 78 percent of the 'Moroccan minority group' in Brussels chose the 'Turkish or Moroccan national identity' over Belgium or Brussels as their central identity category (Phalet and Swyngedouw 2002).

When the use of such categories for self-reference is interpreted as 'identification' with another country (Slootman and Duyvendak 2015), this is often seen as a problem, as a lack of identification with the country of residence is seen to impede immigrants' (and their descendants') integration (Snel, Engbersen, and Leerkes 2006). Being heard to indicate affiliation with another country can lead to exclusion and marginalization, especially in a sociopolitical climate in which the 'native culture' is increasingly framed as being under threat and where feeling belonging, and being 'loyal,' to the nation has become central to being perceived as a rightful or 'deserving' citizen (Spotti 2011). Duyvendak $(2011,92)$ has extensively described this process in the Netherlands, which he calls the 'culturalization of citizenship': "a process in which emotions, feelings, norms and values, symbols and traditions (including religion) come to play a pivotal role in defining what can be expected of a Dutch citizen." Because those feelings are difficult to perceive, Duyvendak suggests, "certain actions become their symbolic stand-ins" $(2011,93)$. In the academic and popular discourse just reviewed, use of the category label Nederlander for self-reference appears to be understood as such a symbolic stand-in.

However, as the vignettes at the start of this chapter suggest, category labels do not mean the same for everyone and in every context. For instance, in the first vignette, Amira differentiated between the categories 'Dutch' and 'Moroccan' on the basis of (dis)obedience to rules, and in the third vignette, Hatice associated being 'Dutch' with being 'weird.' Those examples illustrate that self- or other-categorizations are not always necessarily or straightforwardly related to 'degree of integration,' 'loyalty to the nation,' or 'feelings of (un)belonging.' To appreciate the complex meaning potential of ethnic categories, and to understand what people might gain from selfcategorizing in interaction, it is necessary to focus on interactional data in a context well known to the researcher, in other words, to take a sociolinguisticethnographic approach that takes into account local contingencies as well as 


\section{Nederlanders and buitenlanders}

relations to larger scale processes and discourses. In this dissertation, I build on the close analysis of talk-in-interaction to explore ethnic category labels as signs that, aside from conventional, societal associations, can have locally contingent meanings and indexicalities (Silverstein 2003). By analyzing category label use among the pupils of what henceforth will be called class $3 / 4 \mathrm{~b}$, I will show that the meanings of categories are negotiated - and often contested - among actors in local settings as part of their daily interactions, and that they can vary from one individual to the next, and from one moment to the next.

In addition, I will show that references to categories, aside from their role in the construction and negotiation of (fluid, context-specific, ethnic and other) identities, can also have functions in the organization of talk-ininteraction. Much of the research cited above appears to interpret selfcategorization with an ethnic label as a straightforward indication of an individual's sense of self, or 'identity.' This overlooks the key insight in conversation analysis and different strands of linguistics that turns at talk are (also) social actions (e.g. Schegloff 1996). For instance, making a joke about 'Turks' (as Amira does in the third opening vignette) could function to lighten the mood of an interaction, or positioning oneself as buitenlander may enable a speaker to shift positions of expertise vis-à-vis an authoritative figure. In highlighting those (and other) functions of ethnic category mentions, I hope to provide a nuanced account of what categories mean to, and do for, the people who use them, thereby countering what has been referred to as a "fetishization of ethnicity" (Rampton 2005, 7), that is, the tendency to frame individuals as 'exotic' and 'other' and to read 'ethnic identity' in everyday conversations.

The following research question has guided the research and data analysis:

What do ethnic categories and linguistic resources mean, and do, for the pupils and teachers of South High School?

This question will be answered by means of a number of sub-questions, which will be addressed separately in the data analysis chapters.

1. How did pupils categorize themselves and others, and how did they negotiate the meanings of those categories? (Chapter 3)

2. What were the interactional functions of references to ethnic category labels? (Chapter 4)

3. What role did ethnic categorizations play in interactions involving teachers? (Chapter 5) 
4. What was the relation between categorization practices and the use of different linguistic resources? (Chapter 6)

By means of those research questions, this dissertation will show, i) that widespread national/ethnic categories have various local meanings, ii) that categorizations and the use of linguistic resources contribute to the emergence, maintenance, and negotiation, of local social hierarchies, iii) that categorizations are in addition tied to interactional necessities, and iv) that pupils and teachers not only engaged in negotiations of local social hierarchies, but that they also engaged with wider-spread definitions of categories and linguistic resources, and with the wider societal hierarchies that these definitions support.

An outline of the contents of the dissertation will follow at the end of this introductory chapter. First, I will introduce the theoretical and methodological underpinnings of the dissertation. The specific methods by which I gathered the data, and more information about the participants as well as the context of research, are discussed in Chapter 2.

\subsection{Theoretical and methodological foundations}

In each chapter of data analysis (Chapters 3 to 6), I will introduce the concepts and literature that are relevant to the questions asked in that chapter. This enables the reader to contextualize the findings put forward in that chapter. What follows here is a more general overview of disciplinary traditions and theoretical frameworks that guide the dissertation as a whole.

In this dissertation, I analyze language practices in order to understand social practices, which places the project in the disciplines of (socio)linguistics and anthropology. I therefore start, in 1.1.1, with a short outline of theories at the intersection of linguistics and anthropology. The popular (yet contested) concept of identity, discussed in section 1.1.2, often features in linguistic anthropological work, and is relevant for the dissertation's analyses of social and linguistic stratification through categorization of people and languages. The 'identities' or social categories that the pupils of South High School constructed and negotiated were based on pupils' migration background, which is why section 1.1.3 focuses on the issue of ethnicity. That section summarizes how ethnicity has been approached in the past, which approaches are currently dominant, and it introduces the rationale for the theoretical and methodological approach that I build on, that is, Conversation Analysis (CA) and Membership Categorization Analysis (MCA). Section 1.1.4 then contains an overview of the history and principles of CA and MCA, which is an empirical enterprise, a method of sorts, but is also very closely intertwined 


\section{Nederlanders and buitenlanders}

with theory of social interaction (Peräkylä 2007, 154). The dissertation builds on a combination of CA/MCA and ethnography, which have many things in common, but are also essentially different in some respects. Before introducing those convergences and divergences in 1.1.6, I first expand on the methodological foundations of ethnography in 1.1.5. In a final summary, I recapitulate how all these sub-sections build on each other and together form the theoretical and methodological foundations of the dissertation.

\subsubsection{Intersections of linguistics and anthropology}

In this section, I provide a short overview of linguistic anthropology and sociolinguistics - the fields that are most prominently associated with the study of language and social practices - and the contexts from which these fields of study originate, to then elaborate more on their theoretical and philosophical underpinnings and concerns that tend to be central to them.

Linguistic anthropology as a formal discipline emerged as one of the four branches of anthropology in the late $19^{\text {th }}$ century (the others being archaeological, biological or physical, and sociocultural anthropology) (Duranti 2011). Franz Boas argued that access to language was necessary in order to understand another culture, and not only for practical reasons (i.e. in order to understand informants), but also theoretically, because of the close relations between language and culture (Duranti 1997). The study of language and its relations to culture from within the discipline of linguistics started much later, in the 1950s and 1960s, when some scholars shifted the object of study from 'language' (as a system, or a grammar) to 'speaking' (as an activity). Until then, the dominant paradigm in linguistics had been to study abstract mentalistic grammars that defined the essence of language and humans' capacity for it (Mesthrie 2011). Sociolinguists, conversely, set out to study actual instances of language as used in interaction instead of relying on fabricated or abstract idealized types of language (Coupland 2007).

From that time, particular strands of linguistic anthropology and sociolinguistics have increasingly converged. There are a number of ways in which they have done so, and under different names, including sociolinguistics and linguistic anthropology, but also ethnography of communication, interactional sociolinguistics, and sociocultural linguistics. Although these efforts differ in the degree and kind of attention given to language on the one hand, and social life on the other, a sharp distinction between these fields and related ones that are concerned with "language as a sociocultural phenomenon" (Bucholtz and Hall 2008, 401) is unsustainable given their substantial common ground. I will therefore describe some foundational assumptions that have been central to the study of language, culture, and 
society, and that are relevant to this dissertation. These have not necessarily been developed under the 'disciplinary banner' (Bucholtz and Hall 2008, 403) of linguistic anthropology, but I have nevertheless opted to use that term for consistency and clarity.

Linguistic anthropology involves looking at language not merely as a sign system, where words stand in a one-to-one relation to meanings, and are used primarily to communicate, but instead approaching language as social action. Language is taken as a lens onto social practices, as it is considered one of the prime ways people have of relating to one another. It is seen as an "indispensable medium for the transmission and reproduction of culture and society" (Duranti 2003, 333). This implies that, often, the aim is not to learn about language as much as to learn about social life. Bucholtz and Hall (2008, 405) have phrased the central question as follows: "how does the empirical study of language illuminate social and cultural processes?" Linguistic anthropology has thereby developed as an inherently interdisciplinary field that draws not only from linguistics and anthropology, but also from sociology, education, psychology, cognition, and other fields.

Linguistic anthropology's focus on the linguistic production of culture means that, in many ways, it is the study of speech, but more so, the study of speakers and how they (re)produce identities through language. The term identity itself was not often used until relatively recently, but since the concept has moved from the background into the limelight, now being studied as a topic in its own right, much work within and outside linguistic anthropology has revolved around it. The study of identity is prominent in many disciplines, but it is especially important in linguistic anthropology because, as Bucholtz and Hall $(2004,369)$ argue, "among the many symbolic resources available for the cultural production of identity, language is the most flexible and pervasive." Because this dissertation studies the sociocultural production of the idea that there are different 'kinds of people' (i.e. 'Turks,' 'Moroccans,' 'Dutch people') - or different 'identities' - it is necessary to take a closer look at the concept of identity.

\subsubsection{Language and identity}

As mentioned, although the term 'identity' has only been introduced relatively recently - and has, since then, taken a prominent position in social sciences at large - the study of kinds of speakers has always been central to sociolinguistics and linguistic anthropology. Within sociolinguistics, the variationist approach was one of the first major efforts to study language use as it relates to social identity. The first study of this kind was conducted by William Labov, who researched linguistic variation on Martha's Vineyard in 1963, and later in New 


\section{Nederlanders and buitenlanders}

York City, using a predominantly quantitative approach. Labov established that there was a "regular pattern of socioeconomic stratification of linguistic form" (Eckert 2012, 88), in other words, he found that different ways of speaking could be correlated with speakers' socioeconomic positions and ethnic backgrounds. Furthermore, individual speakers' variation mirrored variation across socioeconomic groups: in more formal situations, for instance, speakers would tend to use variants associated with higher socio-economic groups (Jaspers and Van Hoof 2019). Studies with this approach build on the use of predetermined social categories that are of interest to the researcher (e.g. gender, age, socioeconomic class) and brief contact with speakers who are taken as representative of those categories. A later branch of variation studies replaced the survey approach with an ethnographic approach in order to gain a better understanding of the local dynamics involved in linguistic variation (Eckert 2012). Instead of projecting the macrosociological categories that researchers are interested in onto the speakers, they search for the local categories that speakers use (e.g. 'jocks' and 'burn-outs' in Eckert (1989)) which in turn are still sometimes used to shed light on the macrosociological categories. Thereby, these studies draw relations between local social dynamics from which such categories arise and the use of different linguistic forms.

An often-heard critique on variation studies of these kinds is that they suppose a causal relationship between social identity and linguistic behavior: because of a speaker's membership in a category, they display certain linguistic features (Benwell and Stokoe 2006). Such critiques led to a major shift in theorization of the relations between language and identity, which Eckert (2012) identifies as the third wave of variation study. ${ }^{2}$ This shift came forth from critique on variation studies as assuming causal relationships between social identity and language, but also from increasing critiques on the concept of identity itself. In particular, the concept has been charged with essentialism, that is, it has been said (re)produce assumptions of fundamental similarity between members of social groups and intrinsic differences from members of other groups (Bucholtz and Hall 2004). To avoid such generalizations and essentialism, many contemporary studies of identity have stressed their 'constructionist' approach to identity. This involves seeing it as "a relational and sociocultural phenomenon that emerges and circulates in local discourse

\footnotetext{
${ }^{2}$ It is important to note that these different 'waves' are not clear-cut, differentiated schools of thought where the differences are absolute, and where one 'wave' replaced the last. Studies that fit Eckert's (2012) descriptions of variation studies of the first wave and second wave of variation are still being conducted and add valuable insights to our understanding of language and social life.
} 
contexts of interaction rather than as a stable structure located primarily in the individual psyche or in fixed social categories" (Bucholtz and Hall 2005, 58586).

According to this approach, identities are fluid rather than fixed, multiple, relationally constructed, intersubjectively negotiated, and contextually mutable. The attention is on the ways in which speakers employ stylistic practices to assert an 'identity' or a place in the social landscape, and linguistic variation is seen as social action rather than merely (linguistic) behavior. Attention is thus more on speakers, on how they strategically employ language and other semiotic resources to take stances and index identities, and on what they achieve by employing certain kinds of language and linguistic forms. This approach thus looks at language as well as identity as strategic, flexible, and agentive. 'Identity' does not reside inside a person, as some core of being. A constructionist approach examines how people themselves understand and construct an identity, and how they use it rhetorically to accomplish social action (Benwell and Stokoe 2006). As Benwell and Stokoe summarize it: "Who we are to each other, then, is accomplished, disputed, ascribed, resisted, managed and negotiated in discourse" (Benwell and Stokoe 2006, 4).

But this approach to identity has also been criticized. Brubaker and Cooper $(2000,1)$, for instance, argue that constructionist approaches to identity as fluid, multiple and constructed leave the term without "analytical purchase." They argue that the widespread use of 'identity' - for example in politics and in everyday talk - should not mean that it must be used to analyze such discourse: "the contemporary salience of 'identity' as a category of practice does not require its use as a category of analysis" (Brubaker and Cooper 2000, 5). Doing so, they argue, may (unintentionally) reproduce or reinforce the idea of identity as a reality and thereby reify a notion that it seeks to complicate. An alternative concept such as 'identification,' they argue, "invites us to specify the agents that do the identifying" and "it does not presuppose that such identifying (even by powerful agents, such as the state) will necessarily result in [...] internal sameness [...]" (Brubaker and Cooper 2000, 14).

In this dissertation, I follow Brubaker and Cooper (2000) by focusing on the processes by which boundaries between people are made, unmade, shifted, and negotiated, and thus tend to write about how informants 'identified' themselves. By this, I aim to emphasize that it is not me, as a researcher, who decided to categorize people in those terms, and that I do not see those categories as existent within the world, but that it was they who employed certain terms to describe themselves. The informants of this study - the pupils of class 3/4b of South High School - often used what might be understood as ethnic categories, or categories of descent (Turk, Marokkaan, buitenlander, 
Nederlander) to identify themselves and one another. In the current literature, ethnicity is often understood as a kind of identity. In the next section, I will therefore zoom in on approaches to ethnicity and ethnic identification.

\subsubsection{Beyond constructivism in approaches to ethnicity}

Approaches to ethnicity have undergone developments similar to approaches to (other kinds of) identity as expounded in the previous section. Once, ethnicity was treated as a taken-for-granted and unquestioned category, and the study of it was characterized by a search for essences and definitions of certain 'ethnic groups.' This approach has been called essentialism and is premised on the idea that ethnicity is acquired through birth, represents a given characteristic of the social world, and that members of the same ethnic group are somehow 'essentially' similar to one another and different from members of other ethnic groups (Wimmer 2013). This position has largely been replaced with a more social and processual view of ethnicity, generally referred to as constructivism (or constructionism). This shift is widely attributed to Fredrik Barth, a Norwegian anthropologist, who first introduced the metaphor of boundaries in studies of ethnicity in 1969.

Barth argued for a focus on "the ethnic boundary that defines the group, not the cultural stuff that it encloses" (Barth 1969, 15). He posited that the 'cultural stuff that, in previous work on ethnicity, had been analyzed as more or less objective differences, was actually the result of social processes by which the boundaries of identification between ethnic 'groups' were made, remade, and shifted (Jenkins 2008). So, instead of trying to pin down the supposed content that characterized "discrete groups of people, i.e. ethnic units" (Barth 1969, 9), Barth advocated for a focus on processes by which boundaries between supposed groups were negotiated, maintained, or shifted. Barth's influence in the anthropological study of ethnicity has been characterized as 'particularly germinal' (Lamont and Molnar 2002, 174; see also Jenkins 2008). After him, increasingly, scholarly approaches to ethnicity came to emphasize the processual and dynamic aspects of ethnicity and race (Brubaker 2009). By the 1990s, constructivism had become mainstream in scholarship on ethnicity. Although contrary positions are still expressed, references to ethnicity as 'constructed,' 'contested,' and 'contingent' are now commonplace and "testify to the hegemony of constructivism" (Wimmer 2013, 2). According to Rogers Brubaker $(2009,28)$, "we are all constructivists now." Essentialism, in the meantime, has become loaded with negative connotations (Phillips 2010), and is often associated with racism (Verkuyten 2003).

Constructivism points to the social basis of knowledge claims (Wooffit 2005). It became the dominant paradigm not only in research on ethnicity, 
but in studies of 'identity' more broadly, as mentioned previously. In this work, ethnicity is seen as one particular kind of identity, conceptualized as "the emergent product rather than the pre-existing source of linguistic and other semiotic practices and therefore as fundamentally a social and cultural phenomenon" (Bucholtz and Hall 2005, 588). It is thus not something that lives within an individual, but it emerges in interaction with other people, in processes of differentiation and identification that are specific to contexts, times, spaces and interactants. Ethnicity, like identity, is understood as fluid, multiple, constructed, negotiated, in flux, and contingent on time and space.

There are some risks in this hegemonic constructivist approach to ethnicity, however. Especially Rogers Brubaker (2004, 2009) has been influential in criticizing the constructivist paradigm, signaling a number of problems. He argues that, although constructivism has been an essential and indispensable development in social scientific thought about ethnicity, it has by now become a cliché:

"[B]y virtue of its very success, the constructivist idiom has grown 'weary, stale, flat and unprofitable.' Once an insurgent undertaking, a bracing challenge to entrenched ways of seeing, constructivism has become the epitome of academic respectability, even orthodoxy. It is not that the notion of social construction is wrong; it is rather that it is today too obviously right, too familiar, too readily taken for granted, to generate the friction, force, and freshness needed to push arguments further and generate new insights." (Brubaker 2004, 3)

Not only has the idiom of constructivism become too familiar to generate new insights, but also, at the same time that everyone claims to be a constructivist, much analysis within work on ethnicity reproduces 'groupist' assumptions. 'Groupism' refers to "the tendency to treat ethnic groups, nations and races as substantial entities to which interests and agency can be attributed [and to] represent the social and cultural world as a multichrome mosaic of monochrome ethnic, racial or cultural blocs" (Brubaker 2002, 164). There is an inherent tension between claiming a constructivist stance and still referring to - for instance - "the Catholics in Ireland" or "the Moroccans in the Netherlands" in a way that reifies the idea that they are bounded units with homogeneous members. Constructivism is thus often claimed, but not put into practice. Another problem is that sometimes, constructivism as an epistemological stance is confounded with statements about empirical reality (Wimmer 2013). An adherence to the theoretical or analytical stance that ethnicity is a form of social action and a dynamic process that stems from interactions between people, can fall short of explaining how, and why, it 


\section{Nederlanders and buitenlanders}

nevertheless might be so that participants see it as something innate and unchangeable.

In sum, some key problems with the study of ethnicity are: i) persistent 'groupism' that proposes that the world consists of 'ethnic groups' that are bounded (Brubaker 2004), despite ii) a by now 'clichéd' constructivism that has allowed researchers to routinely claim a focus on dynamic social processes that make up ethnicity, but all the while using groupist language in analyses (Brubaker 2004), both leading to iii) the question of how to explain the persistent idea of ethnicity in our participants' lives without taking it as a category of analysis (Brubaker 2002).

There have been several ways in which researchers have attempted to overcome these problems. Brubaker (2009) identifies two broad strands of work that, in his eyes, have been able to move 'beyond groupism.' The first strand is dynamic and processual approaches that have emphasized how ideas about ethnic 'groups' have changed over time and across contexts, and as such stress the process of ethnic-group-making rather than a state of ethnic-groupbeing (see Brubaker 2009 for an overview). Wimmer (2013), for instance, takes a comparative approach in an attempt to avoid "the Scylla of hyperconstructivism as much as the Charybdis of essentialism" (Wimmer 2013, 3). Comparing the salience of ethnicity in contexts varying from neighborhoods in Switzerland to friendship networks on US college campuses, he aims to find out how it comes to be that the idea of ethnicity matters more in some societies, situations, and periods than it does in others. Another cluster of work overcomes groupist tendencies by focusing on ethnicity (and race and nationhood ${ }^{3}$ ) as a perspective on the world instead of a thing in the world. These approaches are part of what Brubaker (2009) has referred to as a 'cognitive turn' in studies of ethnicity (see also Brubaker, Loveman, and Stamatov 2004). Although much ethnographic and interactionist work explicitly distances itself from cognitivism in favor of discursive approaches (e.g. Antaki and Widdicombe 1998; Edwards 1991), Brubaker et al. (2004) argue that this is based on a limited view of cognitive research as focusing only on a computational, individualistic model of the mind. The cognitive turn, in Brubaker's terms, is "concerned not only with ways of seeing and thinking determined by universal features of our cognitive architecture, but with culturally specific ways in which persons, institutions,

\footnotetext{
${ }^{3}$ Brubaker argues for analyzing ethnicity, race and nationalism together as "a single integrated family of forms" (Brubaker 2009, 22), between which it does not make much sense to differentiate. Even though they might not be (seen as) the exact same thing, there is sufficient overlap in their treatment to warrant seeing them together (see also Wimmer 2013).
} 
organizations, and discourses make sense of experience and interpret the social world" (Brubaker 2009, 32). A cognitivist approach, to Brubaker et al. (2004, 53), would help researchers to be more constructivist and less groupist by "encourag[ing] us to ask how, when, and why people interpret social experience in racial, ethnic, or national terms."

The analyses in this dissertation are inspired by Brubaker's critiques in the sense that I aim to overcome both groupism and clichéd constructivism. I avoid groupism by steering clear of making statements about the 'cultural stuff (Barth 1969) that supposedly defines 'Moroccans,' 'Turks,' or 'Dutch people,' or about what purportedly differentiates them from one another. The scare quotes around these terms are, in fact, intended to prevent them from being understood as my categories of analysis, while they are my participants' categories of practice. Building on Brubaker, these categories are "a key part of what $[\mathrm{I}]$ want to explain, not what $[\mathrm{I}]$ want to explain things with; it belongs to [my] empirical data, not to [my] analytical toolkit" (Brubaker 2002, 165, emphasis in original). At the same time, I avoid clichéd constructivism, in that the constructedness of ethnicity is not used as a concept in, or presented as the outcome of, my research. The pupils of class $3 / 4 \mathrm{~b}$ of South High School treated ethnic categories as something real, also when they disagreed, or were inconsistent, about their supposed implications. In that sense, the idea that ethnicity is constructed is not a satisfying explanation of what I observed. Nevertheless, constructivism has informed my approach to and analysis of the data: I do not think of ethnic categories as an 'objective reality,' but instead believe that they are the outcome of social processes and boundary making. In this dissertation, I ask how the idea of ethnicity is made and unmade in everyday interaction between individuals - which evidently is premised on the notion that it does not exist as an objective reality. Constructivism, in this research, is thus present as a theoretical underpinning and an outlook on social life, and as suggested by Woolard (2008, 434), "not as an afterthought but as a foundation."

This focus on the processes by which ethnicity is made and unmade, and boundaries are shifted, maintained, and negotiated, calls for a particular methodology. In the dissertation, I build on a combination of ethnomethodological approaches (Conversation Analysis and Membership Categorization Analysis) and ethnography. In section 1.1.6, I describe what this combination of CA and MCA on the one hand, and ethnography on the other, entails. Before that, however, it is first necessary to describe both approaches in some more detail separately (CA and MCA in 1.1.4 and ethnography in 1.1.5).

One final comment is pertinent here. Although I refer to the categories used by the pupils in this study as 'ethnic,' they themselves did not use the 


\section{Nederlanders and buitenlanders}

term 'ethnicity' to characterize their categories. If anything, they spoke about afkomst 'descent.' If I am claiming to focus on participants' orientations and explanations rather than my own, what, then, justifies my characterization of those categories as ethnic categories? Firstly, I could have chosen 'national' or 'ethno-national' as a descriptor as well, but I agree with several authors that ethnicity, race, and nationhood can be fruitfully construed as "a single integrated family of forms of cultural understanding, social organization, and political contestation" (Brubaker 2009, 21; see also Wimmer 2013, 7). There is not much sense in trying to draw strict boundaries between ethnicity, race, and nationhood, when they are taken as a perspective on, rather than a thing in, the world. The question of interest is thus not whether the category 'Moroccan' or 'Dutch' is an ethnic or a national category, or what defines 'Moroccanness' or 'Dutchness,' but rather bow these are called into being as things in the world, and when participants come to use these (and not other) categories to do social things such as explaining behavior, challenging power positions in interaction, or making fun of one another, and thereby reproduce an idea that there is such a thing as 'Moroccanness' or 'Dutchness.' The decision is thus first and foremost pragmatic: I use the term 'ethnic categories' reluctantly, not to impose my interpretation of their categories, but rather to clarify the connection to, and facilitate conversation with, other research that refers to those categories as such (although they have also been termed 'national identities' (Koole and Hanson 2002)).

\subsubsection{Conversation Analysis and Membership Categorization Analysis}

Conversation analysis (CA) and membership categorization analysis (MCA) emerged in the late 1960s from a collaboration between Harvey Sacks, Emanuel Schegloff and Gail Jefferson, who were influenced by the early ethnomethodology of Harold Garfinkel and his notion that "social life is a continuous display of people's local understandings of what is going on" (Antaki and Widdicombe 1998, 1). Rather than interpreting participants' behavior, ethnomethodology is interested in how participants themselves display their interpretations of behavior. Social order is taken to be an achievement, which is produced by members of a society who are, in a way, analysts of social situations themselves (Hester and Eglin 1997). They use whatever cues they can -i.e. visual cues, previous experience, or language - to make sense of other people and situations. Ethnomethodology aims to find and analyze those cues: it "treat[s] the obvious as a phenomenon" (Zimmerman and Pollner 1970, 80).

Conversation analysis developed from this outlook on social life and takes as its point of departure that "people accomplish such local 
understanding by elegantly exploiting the features of ordinary talk" (Antaki and Widdicombe 1998,1). Although the focus of conversation analysis is thus on language, CA was not developed in linguistics or communication but in sociology, which was the discipline of Sacks, Schegloff and Jefferson. For them, "language was of sociological significance because it serves as a vehicle for social action and because it can be studied in its particulars" (Sidnell and Stivers 2013, 3). Verbal interaction could be recorded and transcribed, and hence studied in close detail time and again to find patterns and compare similar cases (Francis and Hester 2004).

The foundations for CA were laid, most importantly, by Harvey Sacks in a series of lectures that he gave between 1964 and 1972 at the University of California, published as Lectures on Conversation (1995). The focus in his work was on "how Members, in particular contexts [...], methodically construct their talk so as to produce a possible instance of an action or activity of some sort, and to provide for the possible occurrence next of various sorts of actions by others" (Schegloff 1995, xxvi). Conversation analysts shy away from trying to explain why an action is done in the way it is, or what the speaker is thinking or feeling. It might be tempting to attribute the presence of hedges, pauses, or false starts to the speaker's insecurity, for example, but CA is explicitly "anti-mentalist and anti-cognitivist" (Antaki and Widdicombe 1998, 1). Rather, questions revolve around bow interaction is done, and what a turn at talk achieves in a particular interaction. In Sidnell's $(2013,77)$ words: "Conversation Analysis is meant to be a kind of exploration, the goal of which is the discovery of previously unknown regularities of human interaction." To do this, analysts build collections of phenomena in order to distill the structural from the specific.

In my research, the aim was not to find "previously unknown regularities of human social interaction" (Sidnell 2013, 77). Rather, my analyses are inspired by CA's close attention to how interaction unfolds and how sequences are organized, and I thus build on previous findings in CA as a means to analyze interactions. The other approach that I employ is Membership Categorization Analysis (MCA), which also finds its roots in Sacks' Lectures on Conversation (1995). Since Sacks, however, MCA and CA have developed mostly separately from each other (Hester and Eglin 1997). There is a large amount of work on CA, but MCA has not been developed as extensively (Stokoe 2012). Whereas Conversation Analysis focuses on the systematics of conversation, the organization of talk, and the structure of turn-taking, MCA focuses on how people 'do' person descriptions, and how they recognize them in interaction. Membership categories are "classifications or social types that may be used to describe persons" (Hester and Eglin 1997, 3). In the second opening vignette, Hatice described some children playing in the hallway as 


\section{Nederlanders and buitenlanders}

'Dutch children,' and in the third vignette, Amira refers to Meryem as 'Turkish.' Each of those categories comes with certain associated characteristics ('category-tied predicates' or 'category-incumbent features') and activities ('category-bound activities'), which, to a large extent, are shared between people in a same culture or society ('members'). This makes categories 'inference rich,' that is, "[t]hey are the store house and the filing system for the common-sense knowledge that ordinary people-that means ALL people in their capacity as ordinary people-have about what people are like, how they behave, etc." (Schegloff 2007, 469). Therefore, Hatice and Amira's comments makes sense to their classmates because they share (or, at least, are familiar with) the associations they make between categories and associated characteristics.

Since any person can be categorized 'correctly' in a variety of ways, the correctness of a category is not enough to explain its relevance in a particular utterance. The mere fact that Meryem has a Turkish migration background does not explain its occurrence in this particular context. So, how do people go about selecting one or the other category (Sacks 1995)? Since categories are inference-rich, i.e. they carry associations, the selection of a category evokes those associations (or vice versa: speaking about some associations can evoke a category). The selection of a particular category 'does' something: by evoking certain associations, expectations, or 'knowledge,' it can do interactional work. For instance, by categorizing Meryem's conduct as 'typically Turkish,' Amira could be said to achieve the action of 'teasing.' Categorization can be accomplished without explicitly mentioning the category label, but instead by merely mentioning associated activities or characteristics. Throughout this dissertation, I use the terms 'label(s)' and 'labelling' to refer to a specific type of categorization: that is, when people are explicitly referred to with a category label (such as Marokkaan or Turk). The terms 'category' and 'categorization' refer to the idea of the category and the placement of someone in such a category, whether this is done with or without the explicit use of a label.

MCA and CA developed largely separate from each other, however, and the study of interactional work is mostly perceived as the domain of CA, while categorization is seen as the domain of MCA. Studies that combine categorization and sequential matters have been scarce (Hansen 2005; Stokoe 2012). Stokoe $(2012,279)$ has therefore proposed that if MCA is to survive and "if it is to be respected [...] then a clear approach to the identification of robust, systematic categorial practices is now due." She offers the following guiding principles:

"1. Collect data across different sorts of domestic and institutional settings; collect both interactional and textual materials depending on 
the focus of the study. Data collection may be purposive (e.g. gathering together instances of particular categories in use because of an a priori interest in that category) or unmotivated (e.g. noticing a category's use and pursuing it within and across multiple discourse sites).

2. Build collections of explicit mentions of categories (e.g. man, human, boy-racer, anarchist, teacher, Australian, pianist, prostitute, lesbian, etc.); membership categorization devices (e.g. 'occupation', 'parties to a crime', 'stage of life', 'sex', 'family', etc.); and categoryresonant descriptions (e.g. the descriptions 'she's eighty-nine years old' and 'don't be so testosterony' do not mention categories explicitly but are attributes that 'convey the sense . . . of being deployed as categories'; Schegloff, 2007a: 480).

3. Locate the sequential position of each categorial instance with the ongoing interaction, or within the text.

4. Analyse the design and action orientation of the turn or text in which the category, device or resonant description appears.

5. Look for evidence that, and of how, recipients orient to the category, device or resonant description; for the interactional consequences of a category's use; for co-occurring component features of categorial formulations; and for the way speakers within and between turns build and resist categorizations."

(Stokoe 2012, 280)

These were, broadly, the steps that I have followed to come to many of the analyses in this dissertation, albeit with the difference that I did not collect data across different settings but focused on one setting (with its own different sub-settings), namely class 3/4b of South High School. This is due to the particular combination of ethnomethodological approaches and ethnography in this dissertation (further explicated in Section 1.1.6), and particularly the long-term commitment of conducting ethnographic fieldwork, which inhibited me from including more settings in the analysis.

In my case, the first two steps revolve around defining which categories were relevant in that setting (in the context of this research I have focused on the categories 'Dutch,' 'foreigner,' 'Moroccan,' 'Turk'), gaining an understanding of how they relate to one another (e.g. pupils of class $3 / 4 \mathrm{~b}$ treated the categories 'Moroccan' and 'Turk' as sub-categories of the category 'foreigner'), and analyzing which associations those categories activate for participants (e.g. the category 'Dutch' was often bound to the characteristic of 'following rules,' as illustrated by the first opening vignette of this chapter). Chapters 3 and 5 of the dissertation contain analyses that have come out of 
these first steps. Steps three to five have been applied to arrive at the analyses in Chapter 4 about the interactional functions of category references. In that chapter, I show (for example) how explicitly assuming the category 'foreigner' could enable pupils to assume a (temporary) interactional position of expertise vis-à-vis a person higher up in the local social power structure (e.g. a teacher, or researcher) who was categorized as 'Dutch.' Chapter 6, on the symbolic organization of linguistic resources, does not build on CA and MCA as explicitly as the other chapters, but is nevertheless informed by the same methodological and epistemological underpinnings.

Throughout the dissertation, I have left the discussed categories untranslated (Nederlander, buitenlander, Turk, Marokkaan), or within scare quotes when they appear in their English translation ('Dutch,' 'foreigner,' 'Turk,' 'Moroccan') to emphasize that these categories are defined and given meaning by local actors, and that those meanings may change over time and in different contexts. In that, I follow Wagner $(2017,7)$, who emphasizes: “ I am never making claims about what it means to be Moroccan; I am only making claims about how becoming 'Moroccan' happened in this moment." The scare quotes, then, are meant as a continuous reminder to the reader that the categories themselves, and the characteristics attached to them, were relevant and 'true' to these specific individuals at this specific moment, but will be different in another context, for different individuals, and in another moment. Hopefully, it will also enable the reader to temporarily let go of prior connotations and assumptions surrounding the categories, instead to focus on the understandings displayed by the participants of this study.

In this dissertation, I combine CA and MCA with ethnographic approaches. CA and ethnography show a number of similarities, for instance, they both focus on the regularities of everyday life, emphasize the role of language in the organization of it (Sidnell 2010) and prefer an 'emic,' insider's perspective. In the early days of CA, Sacks was inspired by the ethnographies of anthropologists such as Evans-Pritchard and the way they analyzed what seemed, to the outsider, irrational and strange behavior, but when interpreted and put in context, turned out to be "perfectly rational and logically sound" (Sidnell 2010, 10). Like ethnographic methods, CA and MCA pay close attention to what people do in their day-to-day lives and aim to find order where there was assumed to be chaos. CA was thus influenced, in its beginnings, by anthropology, but the influence also went in the opposite direction. Although conversation had always been of interest to the study of cultural practices and social organization, the rising popularity of Conversation Analysis in the 1970s established conversation as "a proper subject for study" (Duranti 1997, 246) for anthropologists. Many of the terms commonly used in CA (e.g. turn taking, preference, repair, etc. (Duranti 
1997)) have been introduced in other fields, including (linguistic) anthropology. But there are also some fundamental differences between CA and ethnography. Before addressing those differences, and tensions arising from them, in section 1.1.6, I will first describe the main ideas behind ethnography.

\subsubsection{Ethnography}

Taking social life to be the outcome of interactions between people, as intersubjectively constructed rather than something 'out there,' and seeing ethnic (and other) categories as existing in the world as the result of intersubjective processes of boundary maintenance, shifting, and negotiation (as posited in section 1.1.3), begs the question of how those processes take place, and implies the use of a specific methodology to analyze that. In this dissertation, I build on Conversation Analysis and Membership Categorization Analysis (as explained in the previous section) as well as ethnography. In the current section, I focus on the epistemological and methodological principles of ethnography, while in Chapter 2, I describe the specific methods of data collection that I employed to gather the data analyzed throughout the dissertation.

Ethnography aims to study social life as it unfolds in the practices of people in their day-to-day activities, while analyzing the interplay between people's everyday lives and wider structures (O'Reilly 2012). It emphasizes the importance of engaging in direct contact with people for a prolonged period of time in order to gain an insider's perspective, or what Malinowski called the 'native's point of view' (Malinowski 1922 in O'Reilly 2012, 14). Ethnography is not one single method but rather a family of methods that involve spending a relatively long time with research participants and "richly writing up the encounter, respecting, recording, representing at least partly in its own terms, the irreducibility of human experience" (Willis and Trondman 2000, 5). This family of methods may include (among other things) conducting interviews, 'hanging out,' studying documents, taking photos and videos, recording data, engaging in informal chats, and, importantly in this study, participant observation.

Participant observation involves, on the one hand, participating: getting involved in the activities of the informants, joining in, immersing oneself and reaching a level of familiarity with the setting and, potentially, mastery of participants' tasks. Whereas participating is meant to enable the researcher to gain, at least partly, an insider's perspective, observation invokes the more analytic, 'scientific' aspect of the endeavor - keeping analytic distance, making the familiar strange, being critical - and ultimately implies sustaining a 


\section{Nederlanders and buitenlanders}

different relation with the setting than that which the informants themselves have. This inherent tension between participation and observation can be a complicated balancing act, but it also is what gives participant observation its strength: "participating enables the strange to become familiar, observing enables the familiar to become strange" (O'Reilly 2012, 106). These methods enable the researcher to observe and analyze people's (social, cultural, linguistic) practices rather than (merely) relying on what they report about those practices, since actual behavior and reported behavior can diverge in many ways and for many reasons.

Ethnography is typically iterative-inductive, which means that the processes of data collection, analysis and writing take place in a circular fashion, and that they are inextricably linked rather than separate and successive phases of research (O'Reilly 2012). Ethnographic research does not start out with hypotheses to be tested. Usually, the ethnographer has some guiding topics of interest (or 'sensitizing concepts') but allows for freedom to change focus when prompted by the field.

Traditionally, ethnography is associated with anthropology, but today ethnographic methods are used in a range of different disciplines. For the purpose of this dissertation, the ways in which ethnographic methods have been used in linguistics is of interest. In particular, I build on linguistic ethnography. As summarized by Rampton et al. $(2004,2)$ :

[L]inguistic ethnography generally holds that to a considerable degree, language and the social world are mutually shaping, and that close analysis of situated language use can provide both fundamental and distinctive insights into the mechanisms and dynamics of social and cultural production in everyday activity.

Linguistic ethnography pays attention to how language constitutes communicative action, how it is used by people in their daily lives, and how language use is embedded in, and informs us about, wider social structures and ideologies (Creese 2008; Creese and Copland 2017). Paying attention to social as well as linguistic processes, linguistic ethnography aims to answer the questions "What is it about the way we use language that has an impact on social processes? What is it about social processes that influences linguistic ones?” (Heller 1984 in Copland and Creese 2015, 13). In this dissertation, as mentioned earlier, I focus on linguistic and interactional processes and take them as a window onto social life and wider social structures. The next section addresses in more detail the merits of using a combination of CA and MCA on the one hand, and ethnography on the other. 


\subsubsection{Tensions and convergences between CA, MCA and ethnography}

Conversation Analysis has been criticized widely - not only by anthropologists - for a number of reasons. For one, $\mathrm{CA}$ is fundamentally different from other social sciences in its emphasis on 'unmotivated observation': in conversation analysis, the idea is to not start with an interest in pre-defined topics (e.g. 'power,' 'inequality,' 'ethnicity'), but instead to approach data 'openly' to see where it takes you (Sidnell 2008). As Stokoe and Smithson (2001, 238) observe, however, this claim to 'unmotivated looking' has been challenged: "conversation analysts, simply by selecting fragments of talk to analyse, are "motivated" (see also Billig 1999). Other critiques have focused on CA's highly intricate, but nevertheless "rudimentary notion of what constitutes speech" (Duranti 1997, 266) that does not take into account prosody or phonology ${ }^{4}$; its lack of interest in what speakers themselves might have to say about their own behavior, and, particularly in its earlier years, an almost exclusive focus on American English conversation (Moerman 1988).

Most importantly, however, the debate between CA and other disciplines, including sociolinguistics and linguistic anthropology, has focused on the issue of context. In CA, context is conceived to be "actively generated on a turn by turn basis by speakers, rather than as a pre-existing 'frame' within which interaction takes place according to independent rules" (Lepper 2000, 53). In other words, the only 'context' that a conversation analyst is expected to take into account is that which the participants make relevant or orient to in their interaction, and which has a demonstrable effect on how the interaction unfolds (i.e. which has 'procedural consequentiality') (Schegloff 1992). This was fiercely rejected by other social scientists, including Erving Goffman, ${ }^{5}$ who referred to this principle as "the sins of noncontextuality' that is, 'the assumption that bits of conversation can be analyzed in their own right in some independence of what was occurring at the time and place" (Goffman in Duranti 1997, 266). Bourdieu accused ethnomethodologists and conversation analysts of "operat[ing] with the 'occasionalist illusion' that the

${ }^{4}$ Peräkylä $(2007,163)$ notes, however: "Research on the ways in which these features of talk [the rhythm, the amplitude, the pitch and the voice quality of speech] are coordinated with and contribute to the basic conversational organization started in the 1990s (see Couper-Kuhlen and Selting, 1996). Researchers are asking, for example, how prosodic features contribute to turn-taking or the constitution of some basic conversational activities such as openings (Schegloff, 1998) or news deliveries (Freese and Maynard, 1998).”

${ }^{5}$ Goffman was Sacks' teacher at Berkeley. According to Lepper (2000), Sacks' proposition of context is what led to a final break between Sacks and Goffman. 
essence of interaction is entirely contained within it" (Maynard 2003, 69). The conversation analytic approach to context appears to be entirely opposed to anthropological studies that favor the method of ethnography, which is premised on the idea that social practice is situated and does not exist separately from context. For conversation analysts, however, if social structure or other kinds of 'context' are real or important to the participants, it will be reflected in the interaction and will be 'procedurally consequential' (Maynard 2003).

Despite these essential differences, there have been a number of important researchers that have combined ethnography and conversation analysis, the first of which was Moerman (1988), the 'ethnographer-turnedconversation-analyst' (Antaki and Widdicombe 1998, 2) who proposed a 'culturally contexted conversation analysis.' In his work Talking Culture: Ethnography and Conversation Analysis, Moerman studied Thai conversation and compared its sequential organization to conversation analytic findings based on American English. He used CA to study the construction of inequality between Thai peasants and officials, showing how "the materials of all conversation analysis are inextricably cultured" (Moerman 1988, 4) and how "[a]11 meaning is in relation to a context" (Moerman 1988, 7). Moerman thus allowed for - even stressed the significance of - 'context,' whereas conversation analysts had been emphasizing that the only context to be taken into account should be in the talk itself. Combining CA with ethnography, Moerman built on ethnographic knowledge to interpret what was going on in certain interactions, while using conversation analysis to show that that knowledge was put to work in that moment. In his own words: "Whenever I point to 'class' or 'dialect' or 'gender,' I take on the tasks of reporting as an ethnographer on how those things work in this society, and of showing as a conversation analyst that and how they were invoked and used in that very moment of talk" (Moerman 1988, 7).

Moerman's culturally contexted conversation analysis never developed into a full-fledged method of study, but there have been a number of other anthropologists who have combined ethnography and conversation analysis in different ways (Clemente 2013), such as Gumperz (1982), Goodwin (e.g. 1990), Maynard (e.g. 2003), and Sidnell (e.g. 2008), and others who have built on CA though have made the connection less explicit, such as Rampton (2005). All these studies build on CA, but the questions they ask are different from 'pure' CA work. They tend to analyze social phenomena through the study of interaction, rather than limiting themselves to a focus on the workings of interaction itself. In order to do so, ethnographic knowledge is necessary. In Duranti's $(1997,270)$ words: "One cannot speculate about what is known by the participants or the consequences of what is said without having lived in 
a community and having gained an understanding of local norms for sharing information and making claims about what is important and valuable." Ethnographic fieldwork strengthens conversation analytic work in that it enables the researcher to know which questions to ask; to differentiate between 'common' and 'uncommon' interactions between participants; and to acknowledge that all talk is embedded in historical, social, and cultural contexts (Moerman 1988). The analysis of talk-in-interaction together with ethnographic accounts, in Goodwin's words, "permit us to examine how particular practices shape more enduring social relations and are constrained by structures of power that are brought into being in the midst of conversation" (Goodwin 2006, 15). Conversation analysis, in turn, strengthens ethnography in that it provides a way to demonstrate that talk and social organization do not function as separate, independent phenomena or mechanisms but that, rather, talk itself is a form of social organization (Goodwin 2006; Moerman 1988). CA furthermore pushes the analyst to give preference to participants' own concerns and interpretations (which is comparable to the 'emic' perspective pursued by ethnographers), rather than seeing their behavior through the lens of a concept or theory preferred by researchers themselves (Clemente 2013; Goodwin 2006). It offers a systematic and thorough method to show that the topics addressed are not merely the researcher's concern, but also that of the participants.

Membership Categorization Analysis (MCA) generally functions more easily in conjunction with ethnographic approaches than Conversation Analysis does. A crucial principle in MCA is that members can "allude to the category membership" (Schegloff 2007, 470) without actually naming it, but by merely mentioning certain category-bound activities or characteristics. As Evaldsson (2005) points out, to be aware that a participant is alluding to a category without naming it, the analyst necessarily draws on extrainteractional information, especially if the participants' category associations are rather locally specific. With a less restricting notion of culture and context, users of MCA "are entirely happy to work with the margin of cultural familiarity that Sacks allowed himself in a good deal of his early writing, and, in analysing identity, to call upon what they know is conventionally associated with membership of various categories" (Antaki and Widdicombe 1998, 10; see also Stokoe and Smithson 2001). In that sense, MCA is less encumbered with the CA-versus-ethnography problem of contextual knowledge (Evaldsson 2005; Stokoe and Smithson 2001).

Membership categories come with a wide array of associations which may vary for different people. Categories that are familiar to the researcher may have very specific, and diverging, associations to informants. The associations that the pupils of class $3 / 4 \mathrm{~b}$ had with the category Nederlander did 


\section{Nederlanders and buitenlanders}

not always or fully correspond to the associations that I was familiar with, or that are made in many mainstream media, for instance. I would not have been able to recognize allusions to the membership category Nederlander 'Dutch person' by mentions of a characteristic such as 'following the rules,' for instance, if I had not spent an extended period of time with class 3/4b of South High School and learned that they regularly made this association. For membership categorization analysis as it is done in this dissertation, ethnography has thus been indispensable. Long-term participant observation enabled me to become aware of the differences between pupils' and my own categorical associations and pointed me to the questions I ask in this dissertation.

Another benefit of combining CA and MCA with ethnography is - to adapt Rampton et al.'s (2004) appraisal of the advantages of combining linguistics and ethnography - that conversation analysis (CA) and membership categorization analysis (MCA) help 'tie ethnography down' and ethnography helps 'open up' CA and MCA. That is, the use of CA and MCA encourages the ethnographer to focus on clearly defined and delimitable processes (e.g. social categorization in interaction). With its emphasis on recordings and detailed transcripts, data collected and analyzed with CA and MCA can be re-examined and contested by other researchers more easily than field notes of one-time occurrences (Rampton et al. 2004). Ethnography, on the other hand, 'opens CA and MCA up,' in that it enables the conversation analyst to acknowledge and explore the situatedness of any interaction, the impossibility of formulating definite rules about social life, and the inevitable limitedness of focusing on a bounded process such as social categorization in interaction, and it invites necessary skepticism as to the process of making any claim to 'knowledge' or 'reality.'

\subsubsection{Summary of theoretical framework}

In sum, this dissertation situates itself in the discipline of linguistic anthropology, which involves approaching language as social action. Its focus on language practices is intended not to learn about the particulars of a linguistic system, but to illuminate the role that language plays in, and how it influences, social life on a more general level. Much previous work in linguistic anthropology has built on the concept of 'identity' to discuss individuals' sense of self as constructed through language, or it has made links between language use and participants' ascribed 'identities.' Instead of the concept of identity, I will write about 'identification,' or rather, 'categorization,' in order to emphasize that identities are only the products of processes of boundary production, shifting, and negotiation, and do not exist as entities out in the 
world. I focus on those processes, and how they lead to the outcome that people believe its products (being 'identities' or 'categories') are real and consequential.

A large part of this dissertation discusses this for social categories that are often interpreted as 'ethnic categories' or 'ethnic identity,' such as 'Moroccan,' 'Turkish,' and 'Dutch.' Similar to 'identity,' I do not approach 'ethnicity' as an objective reality, but instead focus on the processes by which it is constructed as existent. This theoretical approach calls for a particular methodology: in this case a combination of Conversation Analysis and Membership Categorization Analysis - allowing me to focus on clearly defined and delimitable processes (i.e. social categorization in interaction) and to give preference to participants' own concerns and interpretations - and ethnography - to acknowledge and explore the social, cultural, and historical situatedness of all interactions.

\subsection{Roadmap of the dissertation}

This dissertation will answer the research question 'What do ethnic categories mean, and do, for the pupils and teachers of South High School?' The answer to that question will be addressed in Chapters 3 to 6, which contain the empirical analyses. First, Chapter 3 introduces the category labels that pupils often used, and reviews some of the associations that pupils often made between categories and characteristics and how they negotiated these in interaction. In Chapter 4, I build on tools from CA and MCA to emphasize that category labels did not always or only function to engage in identity work, but that they also had interactional functions among the pupils of class 3/4b. Chapter 5 then takes into account the teachers' roles in categorization practices, by asking how the understandings of categories displayed by teachers compared to, and interacted with, those of pupils. The final empirical chapter, Chapter 6, focuses on the use of languages other than Dutch among the pupils. It describes which languages played a role in daily life at school, and in particular, pupils' and teachers' displayed attitudes toward, and reactions to, different languages. The final chapter contains a short summary of each chapter, highlights the contributions of this study, and suggests avenues for further research.

First, however, it is important to describe the (historical, social, and cultural) contexts in which this research is embedded as well as the fieldwork that I have conducted. This is the topic of Chapter 2. 



\section{Contexts and methods}

\section{Field notes, 25 January 2017}

By the end of Care and Well-being class I join Naomi and her two friends from the other class, Sanne and Eva, who don't really know me yet. Sanne asks me what I do exactly, and I explain that I am spending time with Naomi's class for research. Naomi adds: "It's something about our language." She recalls my first day in class, about 1.5 weeks ago, "the boys were so annoying!" (She must be referring to Justin, who, while I was introducing myself, had claimed to be 25 years old and to have three kids). Sanne, Eva, Naomi and I chat a bit. At one point Eva grabs her phone, then looks at me, and quickly hides it again. "I wasn't on my phone!" I smile and tell her to not worry, I don't care if she is, 'cause I'm not a teacher. "She's not on the enemy side,"Naomi adds with a cheeky grin. At the end of class they ask me if I will be there again on Friday. "We'll chat more then!"

\section{Field notes, 10 April 2017}

Math class. It's early and the pupils are not into it today. It's chaotic, everyone is talking, looking at their phones, no one is paying any attention. The pupils tell the teacher that they don't have their math books with them because they were told they'd have a resuscitation workshop this morning instead of regular classes. About 15 minutes into the class, the teacher pulls up a digital version of the book on the whiteboard and tells the pupils to get their books out. "WE DON'T HAVE THEM!" they repeat, defiantly. The teacher leaves the classroom. Immediately, Ali gets up and rushes through the classroom, collecting all the math books of the people who-in fact-did have them. Some pupils throw their books at Paul. Ali and Paul then throw those books in the walk-in storage closet at the back of the classroom. It's chaos! At that point, Ryan sees me. "Look at her laughing! I forgot there was a teacher here!" I tell him I'm not a teacher, and that yes, this is quite funny. He laughs in surprise and repeats to Ali: "Look at that woman laughing!"

\section{Field notes, 27 February 2018}

Care and Well-being. Ms. Smit is ill, so Ms. Willems has to teach the class alone today. The pupils are settling into their working stations and at desks in small groups, and I am hanging around. Standing at a short distance, I overhear Amine, 
Ben, and Omer - who are sharing a desk - talking. Ben and Omer are nagging Amine - apparently, he was supposed to send them a photo of some assignment, and he didn't. Omer keeps telling Amine to follow him to the locker room, which is about five meters away from where they are sitting. Amine keeps saying that if he does, Omer - who must be about twice Amine's size - will hit him, to which Omer keeps saying, "No, just to talk." They don't notice me eavesdropping, and Ms. Willems is nowhere near. I feel uneasy, and it seems to me that Amine isn't comfortable either. Eventually, Amine gets up and follows Omer into the locker room. In a split second, I decide I have to intervene - what if Omer beats up Amine? So, I bang on the door of the locker room and urge the boys to come out. Omer opens the door, angry, "I am not doing anything, just talking." - "Then do it outside, here in the classroom." Omer is angry; I am too. At the same time, I feel insecure - I am acting like an authority now, and I have tried so hard to show that that's not my role in the school. Omer goes back inside, and again, I tell him to come outside NOW. Leila is watching me, apparently impressed. "Damn, girl!" Eventually, I manage to get them out and we all resume our business.

\subsection{Introduction}

The vignettes at the start of this chapter describe a few moments that I remember particularly well when looking back at my time at South High School. They illustrate, to some extent, my experience of conducting ethnographic fieldwork in this setting: the process of establishing rapport (as in the first vignette), of convincing the pupils I was not a teacher (and sometimes failing - as in the second vignette), and of being confronted with dilemmas as a result of that particular position (the third vignette). In this chapter, I describe the conditions of that fieldwork, and elucidate how it contributed to answering the research questions introduced in Chapter 1.

In the previous chapter, I posited that the meanings of category labels cannot be taken for granted, but that they are highly context-dependent, and that they are negotiated and contested among individuals in a particular context. I argued that, in order to gain a better understanding of the meanings and functions of category labels, it is necessary to pay close attention to the communicative practices of the users of those category labels for an extended period of time, in other words, to adopt a sociolinguistic-ethnographic approach. That is the approach that I have taken in this study, and as a result, the data that I analyze in the following chapters are highly specific to these speakers and contexts. It is thus crucial to have a sense of who those speakers were, and what the contexts were in which their interactions took place. The purpose of this chapter is to provide that background. I will describe in some detail how I selected the fieldwork site (South High School) and the 
participants (the pupils of 3/4b), and why I conducted my fieldwork there to answer the questions that I set out to investigate. I furthermore elaborate on what the research site looked like, and who the individuals were that I met there, that is, the pupils and teachers of class $3 / 4 \mathrm{~b}$. This information provides the necessary backdrop for the reader to contextualize the data and analyses in the empirical chapters to follow.

Before that, however, it is necessary to obtain an understanding of the wider contexts in which this research took place, as no research site stands in isolation from its wider surroundings. Section 2.2 addresses those broader contexts in which I carried out the research and takes the form of three subsections: first, I describe the particularities of the Dutch educational system, the research participants' position in that system, and more generally, I elaborate on my reasons for selecting a secondary school as a research site (\$2.2.1). Thereafter, I describe the fieldwork location in more detail, that is, the city of Venlo and the province in which it is located, Limburg (\$2.2.3). Finally, since the migration backgrounds of many research participants played a prominent role in the interactional practices analyzed in this dissertation, I sketch some context of that migration in the Netherlands and its surrounding discourses $(\$ 2.2 .2)$.

In Section 2.3, I go into the specifics of my fieldwork with the pupils of class 3/4b of South High School in 2017 and 2018. First, I describe how I selected the research site, to then describe the school and the participants in some more detail. This section furthermore contains a description of my daily activities while conducting fieldwork in this school.

The final section of this chapter, Section 2.4, contains reflections regarding ethics and reflexivity. Conducting ethnographic fieldwork entails being in close contact with participants - who, in this case, were minors - for an extended period of time, and reporting on (a part of) their lives. It therefore comes with responsibilities. The third vignette at the start of the chapter describes an example of a moment in which I felt forced to reconsider what had been my main priority during fieldwork - namely to establish and preserve rapport and relationships of trust with participants - and keep in mind the responsibilities that came with my position as an adult immersed in a world of adolescents. At that moment, being a mere participant-observer and siding with the pupils was neither feasible nor ethical, since it was more important to ensure that no harm was done. In Section 2.4, I first describe such ethical considerations that I adhered to while doing ethnographic fieldwork. The second part of that section deals with reflexivity. Because ethnographic fieldwork is such a personal and subjective endeavor, specific to the time, place, and people involved (including the researcher herself), an intrinsic part of doing ethnography is being open about how the research process might 


\section{Nederlanders and buitenlanders}

have influenced results, and reflecting on one's own role in the production of ethnographic and academic findings. The fact that the section on ethics and reflexivity is at the end of the chapter is not meant to suggest that it is of lesser importance. To the contrary, reflexivity is a vital and intrinsic part of conducting ethnographic fieldwork. The section can only be fully contextualized and understood, however, after having gotten an impression of the context and methods of fieldwork.

\subsection{Broad contexts}

\subsubsection{The Dutch educational system}

In the Netherlands, children generally enter the education system at age four with primary school. At the end of primary school, around the age of eleven or twelve, they are referred to different tracks of secondary education according to their supposed academic skills. There are seven different tracks: gymnasium and atheneum (referred to together as vwo - voortgezet wetenschappelijk onderwijs or 'academic secondary education') which prepare pupils for continued education in university; havo (hoger algemeen voortgezet onderwijs or 'higher general secondary education') which prepares pupils for so-called 'universities of applied sciences'; and four sub-tracks fall under vmbo (voorbereidend middelbaar beroepsonderwijs or 'pre-vocational secondary education'). The latter tracks focus on what are often referred to as 'practical skills' more so than on theoretical or academic training (van den Bulk 2011). The four vmbo sub-tracks furthermore differ from each other in terms of time spent on 'theoretical' education versus 'practical' training.

The pupils of class $3 / 4 \mathrm{~b}$ of this study were enrolled in the tracks vmbo basis and kader, which are often referred to as the 'lowest' tracks within the hierarchy. These tracks are highly stigmatized as the 'drain' of the educational system, where pupils end up who are unruly, undisciplined and nonacademically talented (van Daalen 2010). This negative image of vmbo exists in society generally (van den Bulk 2011), as well as among the pupils of class $3 / 4 \mathrm{~b}$, who showed keen awareness of the low prestige of vmbo. They had internalized their assigned position at the lowest end of the educational hierarchy, regularly making comments such as "Don't forget we are basis, we're the lowest!" (Vergeet niet, wij zijn basis, wij zijn het laagste!), when they felt that their teachers demanded too much of them (field notes, 16 February 2017).

It has been well documented that early tracking, that is, the sorting of children into different groups based on an assessment of their academic ability, increases educational inequalities and may reduce mean performance (Hanushek and Wößmann 2006). It contributes to the reproduction of 
structural inequalities - often with strong ethnic and socioeconomic background components (Mehan et al. 1994; Rubin 2006). A recent report in the Netherlands has shown that pupils with migration backgrounds and pupils born to parents who completed vocational schooling themselves are overrepresented in vocational tracks in the Netherlands. Country-wide, 27\% of pupils enrolled in vmbo have a so-called 'non-Western migration background,' compared to $10 \%$ in pre-university education tracks (Inspectie van het Onderwijs 2018). In the class where I conducted this research, seventeen out of thirty-seven pupils, that is 46 percent, had such a migration background - whereas in the municipality of Venlo as a whole only 19.4 percent of children between the ages of 10 and 18 have such a migration background (CBS Statline 2018). Pupils of class 3/4b recognized the overrepresentation of pupils with migration backgrounds in the least prestigious tracks of the school. In the words of one pupil: "Dutch people all got higher recommendations [from primary school teachers] than us foreigners. The foreigners at this school are all in basis and kader" (Field notes, 26 October 2017). Teachers also mentioned that there were less pupils with migration backgrounds in South High School's vwo and bavo tracks.

The early tracking that children undergo in the Netherlands has strong reproductive effects on children of underprivileged backgrounds and with migration backgrounds. Many different factors have been pointed out as major causes of the inequality in, and reproduction of inequality through, the Dutch educational system. The ethnic aspect of this segregation is often attributed to the educational attainments of the parents - a correlation that is also found in children without (perceived or known) migration backgrounds (Huijnk and Andriessen 2016). It has also been argued that primary school teachers tend to have lower academic expectations of children with Turkish or Moroccan migration backgrounds than those without (perceived) migration backgrounds (van den Bergh et al. 2010; see Weiner 2016 for a more extensive overview of literature on inequality in the Dutch educational system), and the same may be true for children who have Limburgish as their first language (Kroon and Vallen 2004). Recent studies in the Netherlands have furthermore pointed to the role of personal relations between teachers and pupils, which appear to be less favorable for pupils with Moroccan migration backgrounds in particular (Thijs, Westhof, and Koomen 2012); language use, where speaking a local dialect and/or immigrant minority language such as Moroccan Arabic or Turkish can lead to lower expectations from teachers (Cornips 2012; Nortier 2009); or the composition of a child's school class, where teachers tend give higher recommendations to individual children when they are in highachieving classes and classes with "relatively few children from low-SES families" (Timmermans, Kuyper, and van der Werf 2015, 474). Earlier 


\section{Nederlanders and buitenlanders}

international work has pointed to the importance of children's, and their families', perceptions of school, arguing that if they do not expect that educational success will translate into, for instance, better job opportunities, they are less likely to work hard at school (D'Amato 1987; see also Van Houtte and Stevens 2010).

Class 3/4b of South High School in Venlo provided a fruitful place to study practices of linguistic and social categorization for several reasons. First, the age of the participants - between 14 and 17 (as further explored in Section 2.3.2) - may have made them inclined to engage in social categorization. Adolescence (as a sociocultural construct, see e.g. Androutsopoulos and Georgakopoulou 2003; Lesko 2012) is a period often characterized as one in which peer group affiliation is of prime importance. Adolescents can be expected to negotiate their own ways of relating to each other, form groups, and (re)create social hierarchies in ways that depend on historical, geographical, cultural and social contexts (Brown, Eicher, and Petrie 1986; Eckert 1989). There is extensive documentation of different kinds of group affiliation among young people (e.g. "jocks" and "burnouts" described by Eckert 1989; "hotrodders" described by Sacks 1995; or "lads" and "ear'oles" in Willis 1981; for more recent studies, see e.g. Androutsopoulos and Georgakopoulou 2003). As Bucholtz and Skapoulli $(2009,4)$ note, youth in the present day "have access to identities that are both global, with respect to transnational, nonterritorial youth culture, and local, by virtue of the particular meanings which the insertion of such forms takes on in local youth-centered linguistic and cultural practices." They might be particularly inclined to adopt and embrace cultural flows because of youth culture's quest for the original and innovative, and the use of internet and mobile phones may have created additional spaces for those flows to circulate more quickly. Youth spend extended periods of time together in schools and categorization is thus likely to occur there. Penelope Eckert has furthermore pointed out that schools foster the development of youth styles and subcultures, since they isolate adolescents in age-segregated institutions and "[exclude them] from meaningful roles in the community and the economy" (Eckert 1989, 15).

In sum, the school class that I researched is an interesting case of a context where youth negotiated local and global scales of reference in their social and cultural practices from a multiply marginalized position, namely as pupils of stigmatized educational tracks, as inhabitants of a peripheralized city (as explored in sub-section 2.2.3), and in some cases as speakers of minoritized languages and/or as descendants of immigrants. 


\subsubsection{Immigration discourses in the Netherlands}

About half of the pupils of the class with which I carried out the research for dissertation had a migration background. Most of these pupils were born to people who had migrated with their parents to the Netherlands from Morocco or Turkey and were thus part of what is often called the second generation. That migration background was central to the categorization practices of the pupils of class $3 / 4 \mathrm{~b}$. In this section, I will give a short overview of the context in which much of this migration occurred, and describe some of the characteristics of, and discourse surrounding, people with Moroccan and Turkish migration background in the Netherlands.

In the 1960s and 1970s, during a period of post-war economic growth, there was great demand for semi- and unskilled labor in the Netherlands. This caused Dutch companies to start recruiting labor in - among other countries - Morocco and Turkey. In the period between 1964 and 1966 a total of 65,000 work permits were granted. In 1975, there were 119,227 so-called 'guest workers' with valid work permits in the Netherlands (van Twist 1977 in Bouras 2012, 43). In Limburg, many of the Moroccan and Turkish migrants came to work in the mines in South and Central Limburg, where the workforce already consisted of a great variety of nationalities (Cranssen 2003). Between 1963 and 1965, between 3000 and 4000 Moroccan men came to work in the Limburgian mines (Bouras 2012). In the second half of the 1960s, when the Limburgian mines started to close, many of the foreign workers found jobs elsewhere in or outside Limburg. The mines still needed a workforce, however, and more men were recruited for the final years of minework (Cranssen 2003). After the mines closed, some of these workers returned to their home countries, but the majority were placed in other companies inand outside Limburg. It is likely that this was the context in which the first Moroccan and Turkish migrants arrived in Venlo, where there were no mines, but several factories that employed foreign workers.

In the 70s, when the need for labor decreased, a recruitment stop was put in place to stop immigration from Morocco and Turkey, and several policies aimed at stimulating migrants to return. Many migrants had already settled, however, and instead of returning, they had their families join them in the Netherlands (Bouras 2013). This caused an increase in research and policy measures to stimulate migrants' participation in Dutch society. When the expectation that labor migrants would return to their home countries still existed, migration policies had aimed at social and economic integration of immigrants in the Netherlands 'while retaining their identity' (e.g. through Arabic or Turkish language classes and subsidies for cultural organizations and festivities) because this was thought to help them with their return. When that 


\section{Nederlanders and buitenlanders}

expectation was proven untenable, policies continued to stimulate ties to the home country - no longer with the idea of return-migration, but because it was seen as the solution to social disadvantage (Bouras 2013; Duyvendak 2011). Other policies focused on migrants' labor market participation, political activity, and educational attainment.

In the late 1980s, critique on the Dutch policy on ethnic minorities increased. A 1989 report of the Scientific Council for Government Policy (WWR) argued that too much attention had been given to multiculturalism and subsidizing cultural organizations, and that as a result not enough progress had been made in terms of people's economic and social integration in the Netherlands (Bouras 2013). This caused intense political debates and a shift of policy aims away from stimulating 'groupness' toward policies aimed at improving individuals' positions in Dutch society. This debate was brought from the political into the public sphere in 2000 by the publication of a series of articles by Paul Scheffer in the newspaper NRC about the supposed failure of multicultural policies in the Netherlands (Rijkschroeff et al. 2005). It was further intensified after two shocking events - the murder of politician Pim Fortuyn in 2002, who had just entered national politics with anti-immigration and anti-Islam standpoints, and the murder of filmmaker Theo van Gogh in 2004 by an Islamic fundamentalist for making an Islam-critical film. Especially Fortuyn had been successful at tapping into feelings of resentment among Dutch people about the growing numbers of immigrants - a discourse that until then had not found a place in political debates due to the country's self-image as tolerant and open (Geschiere 2009).

From this time, social problems increasingly came to be seen as resulting from immigrants' and their descendants' cultural and emotional ties to the country of descent (the same ties that had been stimulated by the government in past decades), and their 'refusal' to be culturally integrated (Bouras 2013; Geschiere 2009). Integration was no longer seen as only a matter of economic, political or educational participation in Dutch society, but also as an emotional matter - the idea that people should 'feel at home' in the Netherlands became dominant. Duyvendak (2011) has referred to this process as the culturalization of citizenship: "a process in which emotions, feelings, norms and values, symbols and traditions (including religion) come to play a pivotal role in defining what can be expected of a Dutch citizen" (Duyvendak 2011, 92). This discourse is dominant not only in reference to recent immigrants, but also to people who have been born and raised in the Netherlands, and even to those whose parents were also born and raised in the Netherlands.

At present, about 24 percent of the Dutch population has a migration background. This includes people who were born abroad and migrated to the Netherlands ('first generation'); people born in the Netherlands of whom at 
least one parent was born abroad ('second generation'); people referred to as having a 'Western,' as well as a 'non-Western' migration background ${ }^{6}$ (Centraal Bureau voor de Statistiek 2019b). About twenty percent of those individuals - almost five percent of the total population of the Netherlands have a migration background in Morocco or Turkey and are, in many cases, (descendants of) the people who came to the Netherlands as labor migrants. Although the majority of people with Moroccan and Turkish migration backgrounds were born in the Netherlands, much popular, political, and media discourse in the Netherlands continues to represent these groups as national 'Others' (e.g. Roggeband \& van der Haar, 2018). They are urged to assimilate to Dutch society and feel 'belonging,' but at the same time, are constructed as Others in much political discourse and media reporting, which accuse them of anti-social attitudes, religious fundamentalism and lack of integration (Buitelaar and Stock 2010). The positioning of these people at the margins of what is imagined as normative or normal 'Dutch' is reflected in language: They are often described in media, political and public discourse as 'Moroccan,' 'Moroccan Dutch,' or - indeed - as having a 'non-Western migration background' (Duyvendak 2011; Verkuyten and Thijs 2010).

Until recently, a common term to refer to people with migration backgrounds was allochtoon. When this term was introduced in 1971 by sociologist Verwey-Jonker in a government report about different groups that had settled in the Netherlands, ${ }^{7}$ it was supposed to refer to all people with migration backgrounds, but increasingly, it came to be used in reference to people with 'non-Western' migration backgrounds, in particular those who

${ }^{6}$ The division between 'Western' and 'non-Western' is used by the Dutch Central Bureau of Statistics (CBS) to differentiate between people from, on the one hand, Europe (but not Turkey), North-America, Oceania, Japan, and Indonesia (i.e. 'Western'), and on the other hand, those from Turkey, Africa, Latin America, Asia, Surinam, the Dutch Antilles, and Aruba (i.e. 'non-Western'), and is based on the supposed cultural similarity of a region or country to the Netherlands. The difference between 'Western' and 'non-Western' thus has a clear ideological basis since it is not centered on geography but on perceptions of cultural traits. That logic leads to the striking categorization of, for instance, Japan as 'Western' and Aruba as 'non-Western' - although, ironically, Aruba forms part of the Kingdom of the Netherlands as an independent country (van der Haar and Yanow 2011).

${ }^{7}$ The report originally spoke about 'Migrants in the Netherlands,' but this was changed to 'Allochthones in the Netherlands' at the request of the government because it did not want to be perceived as an immigrant society (van der Haar and Yanow 2011). 


\section{Nederlanders and buitenlanders}

were positioned as problematic, which in many cases were people (particularly young men) with Moroccan and Turkish backgrounds (Roggeband and van der Haar 2018). Around the turn of the century, the first critiques were voiced about the term allochtoon, its stigmatizing effects, and the way it continued to categorize and exclude people who were born and raised in the Netherlands. In 2016, it was finally abolished from official usage and replaced by 'person with a migration background.' The division 'Western' and 'non-Western' is still widely used, ${ }^{8}$ resulting in peculiar constructions such as a newspaper headline in 2018 that stated that "non-Western vocational students have a hard time getting internships."

Several studies have found that many youth with migration backgrounds declare that they are Dutch ('Nederlands zijn'), but because they are constantly approached as 'Moroccans' or 'Turks' they cannot readily express to 'feel' Dutch (je Nederlands voelen) or to 'be a Dutch person' (Nederlander zijn) (Omlo 2011; Slootman and Duyvendak 2015; van der Welle 2011). The term Nederlander ('Dutch person') thus seems to have become associated exclusively with people who are perceived as having no migration background..$^{10}$ In some of the larger Dutch cities, such as Amsterdam and Rotterdam, it has been found that people with migration background self-categorize in terms of the city ('Amsterdammer') rather than the country (Slootman and Duyvendak 2015; Omlo 2011; van der Welle 2011; Özpamuk 2018). In the words of Slootman and Duyvendak $(2015,160)$ : "For many in the second generation, the local city identity has become a 'mediating' identity, which expresses belonging and can be combined more easily with other dimensions of their identity, such as being Turkish or Moroccan, than an identity as Dutch." Such research has, so far, primarily been conducted in Amsterdam and Rotterdam, however, which are strongly associated with ethnic, cultural, and social

\footnotetext{
${ }^{8}$ In 2016, a report by the Scientific Council for Government Policy (WWR) stated that the binary opposition 'Western' and 'non-Western' no longer "fits within our highly diverse society" (Bovens et al. 2016, 9, my translation). In a recent interview, the spokesperson of Central Bureau of Statistics stated that the bureau is also reconsidering this division (Papaikonomou 2019).

${ }^{9}$ Article published online on 28 August 2018, accessed 6 December 2019: https://www.nrc.nl/nieuws/2018/08/28/onderzoek-niet-westerse-mboer-komtmoeilijker-aan-stage-a1614435

${ }^{10}$ See, for instance, the short opinion piece 'Nederlander, een besmeurd begrip' (Dutch, a tainted term) by Lotfi El Hamidi in NRC (El Hamidi 2018) and pieces by Nizar Mourabit (a pseudonym of Nelle Boer) (Mourabit 2014) and Butter (2019).
} 
diversity. The question arises of how such processes play out in a place that is commonly less associated with diversities, such as Venlo in Limburg, where this research was conducted.

\subsubsection{Social, cultural and linguistic characteristics of Limburg and Venlo}

The data I draw on in this dissertation were collected in a school in Venlo, the Netherlands (see Figure 1 and 2 for maps). Venlo is located in the province of Limburg. ${ }^{11}$ Because this province is an important frame of reference for practices of identification and social categorization for people in Limburg as well as in Venlo, I will first describe the social, cultural and linguistic characteristics of the province in some detail. Afterwards, I address some of Venlo's particularities.

Limburg is often associated with a strong sense of local identity and particular sociocultural and linguistic practices, from the perspective of people in other parts of the Netherlands as well as that of people in Limburg themselves. This imagination of Limburg as distinct from the rest of the Netherlands finds its roots, among other factors, in a long history of shifting political boundaries and a relatively late integration of the province into the Netherlands in the $19^{\text {th }}$ century (for a detailed description of this history, see e.g. Cornips and Knotter 2016). In the present day, the idea of a specific 'Limburgian' way of being is reproduced in daily discourse, regional media and popular culture (Cornips, de Rooij, and Stengs 2012). According to respondents to a survey commissioned by the newspaper De Limburger in 2017, the 'Limburgian identity' is characterized by, among other things, the regional language (according to 96 percent), carnival (96 percent), vlaai (a typical Limburgian pie, 96 percent), and the landscape (88 percent).

From the perspective of people in other parts of the Netherlands, Limburg is perceived as a periphery, and some stereotypes of Limburgians are that they are "warm and spontaneous" (Cornips and de Rooij 2018a, 3), but also less modern than people in other parts of the country. When speaking Dutch, people from Limburg are perceived to have an 'accent,' marked by a sing-song intonation and the so-called 'soft $\mathrm{g}$ ' $[\mathrm{\gamma}]$ in onset position as opposed to the 'hard g' $[\mathrm{X}]$ which is common in the Central and Northern parts of the Netherlands (Cornips, de Rooij, and Stengs 2012; Thissen 2018). It is not only this way of speaking Dutch that makes people from Limburg recognizable linguistically - more so, it is the regional language (or dialects, that is, Limburgish) spoken in this province. Before going into a description

\footnotetext{
${ }^{11}$ There are two provinces called Limburg: Belgian Limburg and Dutch Limburg. Throughout this dissertation, I use 'Limburg' to refer to Dutch Limburg.
} 
46 Nederlanders and buitenlanders

of the sociolinguistic context of Limburg, however, I will describe some of the particularities of Venlo.

Figure 1: Map of the Netherlands with the province of Limburg marked.

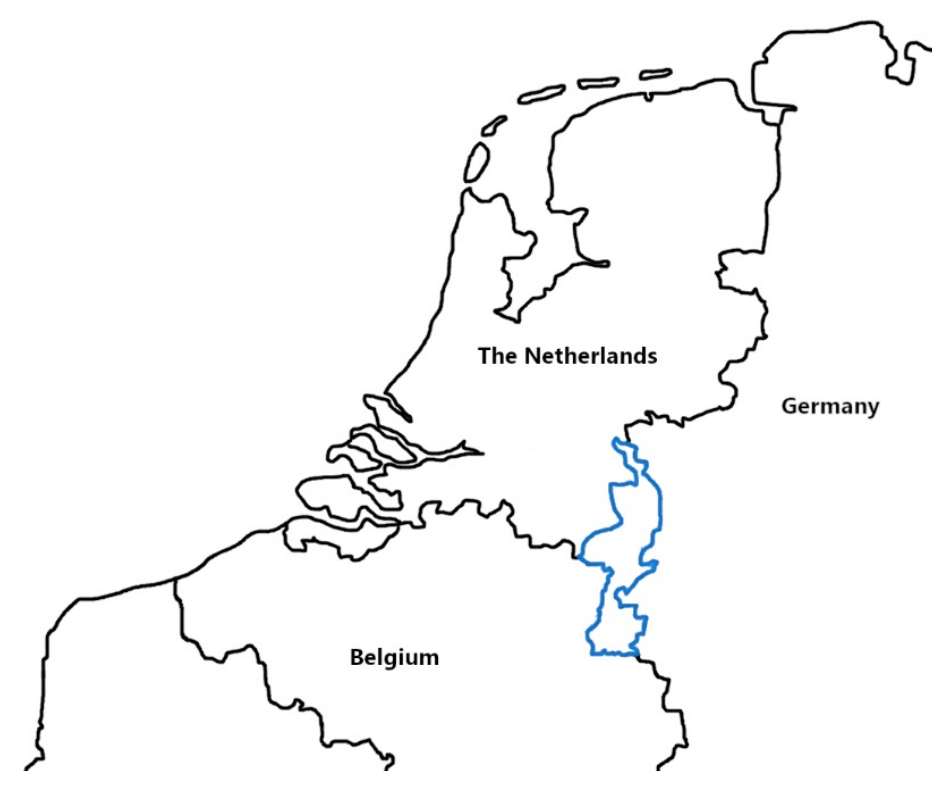

Figure 2: Map of Limburg with select major cities and Venlo marked with a circle.

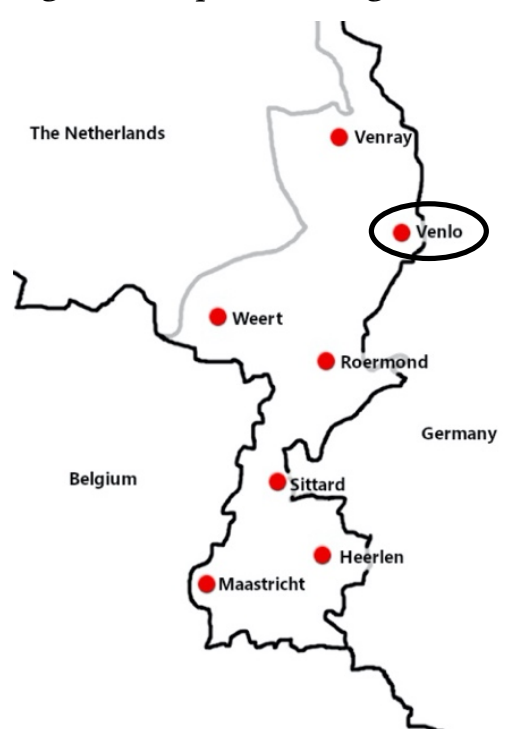


Venlo (see Figure 2) is the second largest municipality of Limburg (after Maastricht, the capital of the province) with a little over $101,000^{12}$ inhabitants when this research was conducted in 2017-2018. The city is located in the Northern part of Limburg, near the border with Germany, as illustrated by the map in Figure 2. While there is a strong imagination of Limburg as distinct from the rest of the Netherlands, there is also an idea that North and South Limburg differ from each other in terms of language, mentality, and attitude (Cornips and Knotter 2016). The long shape of the province (about $140 \mathrm{~km}$ ) contributes to this perceived structural dichotomy. Like the rest of Limburg, Venlo is far removed - physically as well as in the imagination from the socio-economic and political center of the Netherlands, the Randstad. At the same time, it is far removed from the provincial capital Maastricht, which is the socio-economic and political heart of the province. Venlo is nevertheless an important city within its region for trade, transport, and industry, its commercial sector draws many visitors, for instance from nearby Germany, there are a number of institutes of higher education, and it hosts some cultural institutions such as the provincial museum 'Limburgs Museum.' Politically, the city leans toward right-wing conservatism and populism somewhat more than the average for the country: in the national elections of 2017, 20.2 percent of voters in Venlo - as opposed to 13 percent nationally - voted for PVV, a party known for its anti-immigration and antiIslam stances (the leader of which is, in fact, from Venlo).

As mentioned previously, language is central to the construction of a Limburgian identity. Aside from being perceived as having an 'accent' when speaking standard Dutch, people in Limburg are also known for speaking dialects, roofed by the regional language Limburgish. About 75 percent of the inhabitants of Limburg, or 900,000 people, perceive themselves as speakers of Limburgish, and although there is a decline in the number of speakers, this decline is not as stark as that of other dialects in the Netherlands (Driessen 2009). The term Limburgish refers to a wide range of different dialects spoken throughout Limburg, and is the term used in the European Charter for Regional and Minority Languages (ECRML), which recognized it as a regional language in 1997 (Camps 2017). I follow Cornips et al. (2016) in using the following definition of dialect, which applies specifically to Limburg and emphasizes the power differences that a differentiation between 'dialect' and 'standard Dutch' implies:

${ }^{12}$ This number includes the inhabitants of the city of Venlo as well as smaller villages around it, such as Tegelen and Blerick, which are counted within the municipality of Venlo. Children from those villages usually attend secondary schools in the city of Venlo. 
A dialect is the non-official means of communication for a community or region; it has limited currency and is passed on without educational institutions, in the informal privacy of the home situation. It is often oral and rarely written, its usage is often limited to homely matters of family and community life.

(Leerssen 2006 in Cornips et al. 2016, 191)

Speakers of Limburgish often refer to their own variety by the location with which it is associated: The dialect spoken in Venlo is called Venloos; that in the neighboring town of Tegelen Tegels, et cetera. As illustrated by those names, Limburgian dialects are highly enregistered as linked to places, and speakers have a strong dialect awareness, that is, they link the different varieties to the places in which they are perceived to originate (Cornips et al. 2016). Another common way used by speakers to refer to Limburgish is plat. ${ }^{13}$

Much recent sociolinguistic research has focused on the significance of Limburgish for the construction of Limburgian identity (e.g. Camps 2018; Cornips, de Rooij, and Stengs 2012; Cornips et al. 2016; Makkinga-Clijsen 2017; Thissen 2018). Limburgian dialects enjoy high informal prestige, as people of all socioeconomic backgrounds and levels of education speak them (Kroon and Vallen 2004). It is important to note that Limburgian dialects may differ from standard Dutch on all linguistic levels (de Schutter and Hermans 2013). There are also large differences between the dialect groups within Limburg, especially in terms of phonology and vocabulary. Dialect as it is spoken in Venlo (i.e. Venloos) is considered a transitional dialect, since it displays some elements from dialects as they are spoken in more Southern parts of the province, as well as elements from more Northern parts (Keulen et al. 2007).

The imagination and common description of Venlo (and Limburg) as characterized by expressions of 'the local' emphasized so far in this section is not the full story, however. Themes such as those explored in this dissertation - related to social, linguistic, cultural, or ethnic diversity - are often researched in large metropolitan centers such as (in the Netherlands) Amsterdam or Rotterdam, but such diversity also exists in peripheralized areas such as Limburg. For instance, 28.4 percent of Venlo's inhabitants have a migration background compared to 23.6 percent in the Netherlands as a whole (Centraal Bureau voor de Statistiek 2019a). With 12.1 percent, Venlo is Limburg's

\footnotetext{
13 'Plat' stems from 'Platdeutsch.' In Limburg it is perceived to be a neutral and matterof-fact way to refer to the local dialects.
} 
municipality with second most inhabitants with a 'non-Western migration background. ${ }^{14}$ The flows of people, ideas, technologies, and goods associated with globalization (Appadurai 1996) can be expected to not only have altered daily life in large urban centers, but also in places such as Venlo (Wang et al. 2014). Some evidence of this in relation to language in Limburg has been presented in previous sociolinguistic research by, for instance, Cornips (2020a) and Thissen (2018). Cornips (2020a) observes that although many people with migration background in Limburg acquire local dialects, many are not comfortable using dialect because their authenticity and legitimacy as dialectspeakers is often contested. Thissen (2018) describes how, when people who are not perceived to look 'typically Limburgian' (e.g. because of their skin color) use dialect in conversation, they are often answered in standard Dutch. These findings demonstrate that language practices are an important means of social in- and exclusion in Limburg, and that ideologies of dialect purity as rooted in historical knowledge and intergenerational transmission can lead to a perception of people - in particular people with migration background and/or who are not white - as non-legitimate speakers of dialect.

\subsection{Fieldwork context and methods}

The data I draw on in this paper were collected during nine months of ethnographic fieldwork in South High School (a pseudonym), in Venlo, the Netherlands. Ethnography is "a family of methods involving direct and sustained social contact with agents, and of richly writing up the encounter, respecting, recording, representing at least partly in its own terms, the irreducibility of human experience" (Willis and Trondman 2000, 5). Ethnography is characterized by its bottom-up approach, where theory and questions emerge from data rather than vice versa. It is, in other words, iterative-inductive: it does not begin with a hypothesis to be tested, but rather with a topic of interest, a context, or 'sensitizing concepts' (Blumer 1969). In my case, I arrived at South High School with an interest in what I broadly defined as practices of identification, or constructions of the self and others,

${ }^{14}$ The logic behind the categorical distinctions made by Centraal Bureau voor de Statistiek between people with 'Western' and 'non-Western' migration backgrounds is not based on geography (e.g. the continent of Asia is counted as 'non-Western,' but Japan and Indonesia are counted as 'Western'), but rather on the perceived cultural and socioeconomic position of that country. If the culture of a person's country of descent is considered to resemble 'the Dutch culture,' they are counted as 'Western.' Hence, 'Western' functions as the unmarked category, and non-Western as the marked, and often problematized, category (van der Haar and Yanow 2011). 
the role of language(s) in such processes, and the ways in which those unfold in a peripheralized city like Venlo. When I started my fieldwork at South High School, I noticed the frequency with which pupils of class $3 / 4 \mathrm{~b}$ used the category labels buitenlander, Turk, Marokkaan and Nederlander in their daily communicative practices, after which I decided to focus on social and ethnic categorization, specifically. Data collection, analysis, and writing were thus "not discrete phases but inextricably linked" (O'Reilly 2012, 30). The precise topic and research questions of this dissertation came forth from the context in which the research was conducted, and empirical data led to a theoretical interest rather than vice versa.

In the following, I first elaborate on how I came to do research at South High School, and with class 3/4b, specifically. The section thereafter (2.3.2) provides more details about the pupils that participated in the study. In section 2.3.3, I discuss my daily activities during fieldwork, that is, I explain how I collected the data on which this dissertation is based.

\subsubsection{Selection of research site}

At the start of my $\mathrm{PhD}$ project, I approached several secondary schools in Venlo, explaining that I was looking for a school to conduct research about practices of identification and the use of different languages among adolescents. South High School was the first to reply positively. I got in touch through email with the Dean of the vocational tracks (vmbo), who invited me to come to the school for a meeting a few days later. That day, he gave me a tour around the school building, and we spoke about my interest in diversity in terms of cultures and languages. He was strikingly open and positive about the ideas I had for my research, and when I explained I wanted to observe just one class, he suggested that if I was interested in cultures and languages, $3 \mathrm{~b}$ would be a good group. In his words, it was "a very diverse class, with nice pupils who are not too rowdy so the teachers will probably also be okay with you joining them" (Field notes, 20 December 2016). After our conversation, the Dean discussed my proposal with the school's board and director and within about a week, everything had been arranged. Two weeks after my meeting with the Dean, and two months after starting my PhD, I began fieldwork at South High School.

South High School is part of an overarching organization that runs three secondary schools in Venlo. These schools have between 1450 and 2500 pupils and cater to pupils from Venlo and surroundings. They offer the entire spectrum of educational tracks, from pre-university tracks (vwo) to vocational schooling (vmbo). The symbolically potent division between 'vocational' and 'academic' education (which was described previously in section 2.2.1) is 
visible in the architecture of South High School: pupils of pre-university education (vwo) and higher general secondary education (havo) use one side of the school, and the other half of the school is where the pupils of the four vocational tracks (vmbo) have their classes. The pupils in those different tracks hardly meet each other at school: the only space they share, the canteen, is used by them at different times. Pupils as well as teachers perceived havo/vwo and vmbo tracks, and the pupils in them, as essentially different. They had a view of havo/vwo pupils as more organized, less rowdy, and easier to teach than vmbo pupils. Different tracks were, in sum, not simply seen as 'different' but were hierarchically organized. Corresponding to a wider national discourse (van Daalen 2010), vwo was deemed 'higher' than vmbo, and within vmbo there were 'higher' and 'lower' levels too.

\subsubsection{Class $3 / 4 b$}

During my meeting with the Dean of the vmbo tracks of South High School, he had recommended that I would conduct my fieldwork with the pupils of what at that time was called class $3 \mathrm{~b}$. I followed his recommendation and started fieldwork on 9 January 2017, a few months into the pupils' third school year. I stayed until the end of that school year at the end of June. I then returned at the end of September 2017, in the next school year, when most pupils had transitioned into class '4b,' and stayed until mid-March 2018, just before their final exams. Some pupils that I got to know in class $3 \mathrm{~b}$ had not transitioned to class $4 \mathrm{~b}$, and some other pupils had not passed their final exams the previous year and had to re-take year four, joining class $4 \mathrm{~b}$. The composition of the group had thus changed, but only minimally. Throughout this dissertation, 'class $3 / 4 b$ ' refers to the thirty-seven pupils that I got to know during their third and fourth years of secondary school.

Class 3/4b was a vmbo basis class. During vocational classes, which represented a large part of their schedule, they were furthermore joined by pupils from vmbo kader. These tracks are the sub-tracks that are considered the 'lowest' of all vocational education tracks, where most time is spent on vocational classes and least on 'academic' subjects (see section 2.2.1 on page 38 for more details about the Dutch educational system and the place of class $3 / 4 \mathrm{~b}$ in it).

At the beginning of fieldwork, the youngest pupil of class $3 / 4 \mathrm{~b}$ was fourteen years old, most pupils were fifteen, and some were about to turn sixteen. The next year, most were sixteen and the oldest pupils were seventeen years old. There were 18 male pupils, and 19 female pupils. This was the first year that these pupils were put together as a group in this composition, and not all pupils knew each other. As a result, the group consisted of some smaller 


\section{Nederlanders and buitenlanders}

cliques of friends that did know each other from having been in the same class in the previous years, and in some cases, from having attended the same primary school. Such cliques sometimes changed throughout the year, as friendships were forged, and others dissolved. The atmosphere in the class, as I experienced it, was generally rather peaceful. There were the occasional fights between pupils, some confrontations between pupils and teachers, but the pupils largely had an air of acquiescence about them. Most of them treated school as a necessary hurdle to pass. They did not show particular interest in class materials, and most just seemed to want to get good enough grades to pass on to the next year and then graduate. They said they found school, and many of their classmates (outside their own cliques) 'boring,' and were much more excited about their phones and social media, boyfriends and girlfriends, joking and having fun with each other, gossiping, deciding how to spend their spare time, or getting and keeping part-time jobs. Some of these interests will show from the interactions throughout the dissertation.

Most pupils had been born in Venlo or one of the small villages nearby. Twenty out of thirty-seven pupils had no (known or self-reported) migration background; eight pupils had a migration background in Morocco; five in Turkey; and four in Bosnia, Afghanistan, Gabon, and the Dutch Antilles, respectively. With the exception of two pupils who had been born outside the Netherlands, most pupils with migration backgrounds were born to parents who had also been born in the Netherlands or who had moved to the Netherlands as young children. The majority of these facts about the pupils (migration background, age, hometown) I found out through informal conversation, either when it came up between myself and the pupils, or by hearing pupils mentioning it to each other. I did not use a survey or questionnaire to gather these details, as I wanted to get to know them in a way that would be similar to how they got to know each other and to focus on the information that they brought up in regular interactional contexts. This means that I do not know everything about all pupils. For instance, I do not always know for how long their family had lived in Venlo or Limburg; whether their parents or grandparents migrated to the Netherlands; or which languages pupils spoke at home. Such facts about their migration backgrounds did not seem to affect the categorization practices that I analyze in this dissertation, however.

Because the pupils were in the two most practically-oriented vocational education sub-tracks (vmbo basis and vmbo kader), they spent about half their school time in vocational classes according to the specialization they had chosen. Some of class 3/4b's pupils were in the specialization 'Mobility and Transport' (covering mechanics of cars and other vehicles), and the others were in 'Care and Well-being' (covering service-industry related skills, such as 
cooking, hairdressing, first aid, taking care of children or elderly, etc.). The distribution of pupils over those specializations was highly gendered: there were only male pupils in Mobility and Transport, and all the female pupils plus a few male pupils had chosen Care and Well-being. For some, this choice reflected their personal interests or future ambitions - for instance, Nouria wanted to study to be a dentist's assistant - and for others, it seemed like the easiest specialization to choose. It also reflects pupils' rather traditional gendered expectations, as one pupil said: "If you're a girl, you should just be in Care and Well-being" (Field notes, 16 June 2017).

At the start of my fieldwork, I alternated between joining Mobility and Transport classes and Care and Well-being classes during vocational class hours. However, I soon realized that by attending classes in both specializations, I did not establish a full routine in either of the classes, and I decided to 'be there' fully with one group even if at the expense of getting to know the pupils in the other group equally well. I chose to follow the Care and Well-being group more closely, since my presence, as a female researcher in the predominantly male context of Mobility and Transport, seemed to make the Mobility and Transport pupils and some of the teachers uncomfortable, and thereby made me uneasy too. In Care and Well-being, on the other hand, it was easy to become part of the daily routine. This decision meant that I got to know the pupils in Mobility and Transport much less than I did those in Care and Well-being, but it also meant that I got to know those eighteen pupils ( 14 female and 4 male) rather well. It furthermore means that I have more data from interactions between and with female pupils than between male pupils. Although gender is not the main focus of the analyses in this dissertation, it has thus certainly influenced some aspects of my data collection, and potentially some of the results that I present as well. When it comes to analyzing negotiations of local as well as wider societal social hierarchies through categorization, as I do in this dissertation, gender categories are undoubtedly highly relevant and intersect with other categorizations to determine the social positioning of these pupils. It is not merely membership in (for instance) the category 'Moroccan' that is relevant to how society perceives the pupils in this dissertation, and how they perceive themselves, but it is also always potentially some other categories, including that of gender. Furthermore, feminist strands of conversation analysis have argued that gender can be shown to be sequential and relevant in talk-ininteraction even if gender categories are not explicitly mentioned (Speer and Stokoe 2011). The pupils in this dissertation can be seen to orient to gender categories, for instance, in Example 3.6 (on page 82) where a number of pupils discuss whether or not it is allowed in Islam to get one's ears pierced and point to the example of "the wives of the prophet" within this discussion, or in 


\section{Nederlanders and buitenlanders}

Example 4.1, page 105, where a pupil and I discuss whether or not his father has authority over him. Nevertheless, empirically, negotiations of gender categories were less salient in the interactional practices that I observed among the pupils of class 3/4b than the categories 'Moroccan,' 'Turk,' 'foreigner' and 'Dutch.' Analyses of ethnic categorization are therefore at the forefront in this dissertation. This is not to suggest that gender (or other) categories were irrelevant, but that they are beyond the scope of this dissertation.

\subsubsection{Daily activities during fieldwork}

During the fieldwork, I gathered most data by using the method of participant observation, which in my case meant spending prolonged periods of time with my informants. This served to become familiar with the context and gain insight into the participants' behavior by means of observation rather than by asking them about it. In this section, I describe these methods in more detail.

During my time at South High School, I participated in regular school days: I arrived at school around 8:00 in the morning, started classes with class $3 / 4 \mathrm{~b}$ at $8: 15$, and stayed throughout the day to accompany them to their classes. During the first two months of fieldwork, my data collection consisted of taking extensive fieldnotes of everything I observed during and between classes. First, I wrote those notes by hand. Whenever I wrote something down this way, however, pupils would try to find out which of their behavior had caused me to take notes - often hypothesizing that it was because someone had sworn, shouted, or misbehaved in some way. When I switched to notetaking on my tablet, this changed. Pupils used tablets or computers in many classes too and my use of one did not stand out. Furthermore, using a tablet disabled them from distinguishing between me writing field notes or doing other things such as reading the newspaper, and they seemed to be less curious as to what I could be writing about. An additional advantage was that it took me significantly less time to take notes this way and expand on them later.

After two months of detailed notetaking, I started carrying an audiorecorder with a lapel microphone attached to my clothing. This served to be able to re-play interactions later, and then closely analyze them by using conversation analysis and membership categorization analysis. Furthermore, not having to write down what was being said enabled me to pay close attention to visual information such as body position, gaze, et cetera, which complemented the audio recording. I only started recording two months into the fieldwork in order to give the pupils time to get used to me first, before potentially scaring them off or making them uncomfortable by recording immediately. As I explain in more detail in the ethics section (Section 2.4), I informed the pupils, their parents, and teachers about the recorder and gave 
them the opportunity to withdraw from the study (which the parents of one pupil did). I used a small recorder that fit into my pocket, which enabled me to take it with me wherever I went. I recorded almost all classes, amounting to a total of about 140 hours of recordings. I complemented those recordings with field notes, in which I wrote down what I observed and highlighted important events during the day with a time stamp in order to return to the recorded version of the event later. In the fieldnotes, I also wrote down my own moods and feelings, as they have the potential to color interpretations of events during the day.

About half of the schedule of the pupils was taken up by vocational classes, that is Care and Well-being classes in the case of the sub-selection of eighteen pupils that I followed most intensively. About two-third of the time that I spent at South High School (202 class hours out of 333 class hours in total - a class hour was 50 minutes) was in such vocational classes. Care and Well-being classes were much less rigidly structured than regular classes; they were very interactive and there was plenty of opportunity for pupils to interact with each other, and for me to interact with them rather informally. During Care and Well-being, the pupils learned skills related to hairdressing, cooking, and caring for people with disabilities, elderly people, and children. This happened through a combination of book assignments and practical assignments with worksheets. Every couple of weeks, pupils rotated between different 'workstations' in groups of two to six pupils: some would be working in their books at the desks, and others would practice assignments, such as hairdressing or first-aid. The classroom was very large so as to accommodate the wide range of assignments: apart from regular desks, it included four kitchen units, a hospital-type bed, a crib, hairdressing chairs, washing machines, a reception desk, and more. During these classes, I usually joined different work stations for a while, sometimes helping pupils with assignments or being a 'dummy': I have had my hair done by pupils, I was bandaged, manicured, I pretended to choke for them to practice the Heimlich maneuver, I have played an injured person on the street, I have been henna tattooed, and so on. During these classes, I often engaged in informal conversation with pupils, but mostly I observed and listened, while taking notes and making audio recordings.

The format of pupils' regular classes (e.g. math, biology, Dutch, English, etc.) was more traditional. Pupils sat at desks, which were arranged in three rows of paired desks, and the teacher's desk was in front. Every class took place in a different classroom, so between classes all pupils (and most teachers) had to change rooms. When possible, I would sit somewhere at the back of the classroom between the pupils to be in a better position to observe, but sometimes other pupils had already taken those seats and I sat in the middle 


\section{Nederlanders and buitenlanders}

or front. Most pupils tended to prefer to sit at a pair of desks by themselves, and like them, I thus often sat alone at two desks. During these classes, I mostly observed and took field notes while also recording. Sometimes, teachers would ask me to keep an eye on the class while they went out, for example to make copies. At first, this made me uncomfortable as it put me in the position of an authority vis-à-vis the pupils, but I quickly noticed that I could refrain from intervening. I had told teachers about my aim to show pupils I would not behave like a teacher, and I interpreted teachers' requests as one of keeping an eye out that pupils would not harm each other, rather than to check whether or not they copied assignments from each other. Although it can be difficult to manage relations with pupils while also establishing rapport with teachers (e.g. Russell 2005), the teachers at South High School generally seemed to appreciate, or at least accept, my presence in the classroom. They sometimes jokingly admonished me for chatting with the pupils and "keeping them from their work," but as I deduced from our informal and friendly interactions between classes and during breaks, they did not really mind my presence.

I spent breaks between classes in the teachers' lounge. Here, I did not make audio-recordings but took field notes afterwards. I had not intended this to be a context where I would gather data, and I had not asked for permission to record there, but being there served as a way to get to know, and build rapport with, teachers. It helped them to get to know me, too, and it gave me important insights into the attitudes of teachers toward their job and the ways in which they talked about their pupils, and therefore form part of my analyses particularly in Chapter 5 of the dissertation.

I cannot say that pupils or teachers ever saw me as 'one of them' - there were too many differences between us, including but not limited to, for example, our age and daily activities (I go into more detail about my positionality in Section 2.4) - but my presence gradually became ordinary. I became a fly on the wall, so to say. During the initial period of fieldwork, my main aim was to establish rapport with the pupils, as well as with the teachers. I found it important that the pupils understood that, although I was an adult in a secondary school, I did not have the role of a teacher. Especially in situations where a pupil broke some school rule, and looked at me expecting to be reprimanded, I emphasized my lack of authority. With teachers, I used the break time to socialize. As the fieldwork progressed, I no longer went to school every day but started coming three or four days a week, and spent the other days expanding on field notes, coding, transcribing, reading, and doing some initial data analysis which, in keeping with the iterative-inductive approach, informed the development of the focus of my fieldwork. 
One method that is often subsumed under ethnography, which I only used a few times during this fieldwork, is interviewing. I conducted two informal interviews of about twenty minutes (during breaks between classes) with teachers, for whom I had some specific questions. One of those interviews was with Ms. Jansen, the head-teacher of the class who knew many of the pupils rather well, to ask her for her opinion on some of my observations of the class. The other short interview was with Mounira, an intern, about her experience and thoughts about the pupils. I also conducted a makeshift focus group with five pupils during a class, when the teacher (Ms. Jansen) allowed me to take them apart for some questions I had. This took the form of an informal conversation of about twenty minutes. More details about that informal focus group follow in Chapters 3 and 4, where I analyze some interactions that occurred during it. Most of the time, however, I did not organize separate moments of interviewing. Although interviewing has many advantages, and it may be an indispensable method for certain research contexts, it is also "highly unusual, as communicative events go" (Briggs 1986, 2). Interviews tend to create an artificial communicative space, a separate speech event, different from the communicative norms and practices of regular life. I found that the more informal context of the classroom, during and inbetween school activities, was more suitable to find answers to the types of questions I pursued and with these participants. I did so by listening in on, and sometimes participating in, pupils' informal conversations about topics of their own interest, while sometimes asking for clarification or more elaboration. Especially in vocational classes there were enough occasions to talk with pupils and ask questions in an informal, relaxed sphere where conversation could flow naturally. This furthermore enabled me to be led by their topics of interest, and as such get a sense of what they discussed with each other (such as relationships, social media, gossip, summer holidays, food, spare time, fashion, etc.), rather than pursuing what I wanted to know as might happen in a more formal interview. I was interested in how they interacted, and in what would come up in their conversations, and by avoiding leading conversations myself, I aimed to keep the focus on their interactional practices.

In order to deal with the large amounts of data I amassed in this way, I spent afternoons after coming home from South High School fleshing out field notes, uploading the notes as well as the recordings into the data analysis software QSR NVivo, and transcribing the recordings of the moments that I had marked as interesting in my field notes. On days that I did not go to school, I coded the notes and transcripts according to topics of interest. These topics were very broad at the start, as I had come to school with general interests rather than highly specific questions, as is common in ethnographic 
fieldwork. From the moment I became interested in categorization in ethnic terms, I started paying extra attention to interactions in which pupils or teachers used the terms Marokkaan, Turk, buitenlander and Nederlander, Hollander or tatta. This resulted in a collection of 265 recorded and transcribed interactions in which pupils (and sometimes teachers) made references to ethnic category labels, amounting to almost two references for every hour of recording. Analyses of that collection form the basis of this dissertation. Unless signaled otherwise, the interactions that I analyze in this dissertation were selected because they were typical and representative examples of common interactions in this context.

The particular selection of the school context to gather data means, of course, that there are some important parts of the lives of 3/4b's pupils that I have not been able to take into account in my analyses - most prominently, their lives outside school and their online lives. By excluding those, I do not mean to imply they are not relevant. Practical limitations were the main reason for focusing on school life (and not home or leisure time), and face-to-face (and not online) encounters. I gathered an enormous amount of data by 'just' being at school with the pupils for an extended period of time and could not have analyzed much more data by myself in the limited time of a $\mathrm{PhD}$ trajectory. My arguments throughout this dissertation are thus only based on that limited portion of pupils' daily lives in the school that I was able to observe.

\subsection{Ethics and reflexivity}

\subsubsection{Ethics}

As a researcher using ethnographic methods, my main guiding principles in terms of ethics have been those of the American Anthropological Association (AAA). The Principles of Professional Responsibility (PPR, that is, the ethics statement) of the AAA states:

"As a social enterprise, research and practice always involve otherscolleagues, students, research participants, employers, clients, funders (whether institutional, community-based or individual) as well as non-human primates and other animals, among others (all usually referred to as 'research participants' in this document). Anthropologists must be sensitive to the power differentials, constraints, interests and expectations characteristic of all relationships. In a field of such complex rights, responsibilities, and involvements, it is inevitable that misunderstandings, conflicts, and the need to make difficult choices will arise. Anthropologists are 
responsible for grappling with such difficulties and struggling to resolve them in ways compatible with the principles stated here."

(American Anthropological Association 2012)

The principles laid down by the AAA are: do no harm, be open and honest about your work, obtain informed consent and necessary permissions, weigh competing ethical obligations to collaborators and affected parties, make your results accessible, protect and preserve your records, and maintain respectful and ethical professional relationships. In this section, I elaborate on the ways in which I adhered to those principles. To ensure that my research design was up to standard, I furthermore voluntarily submitted it to the Ethical Review Committee of Maastricht University and received their approval. ${ }^{15}$

The principle to do no harm was always at the core of the decisions surrounding my fieldwork. Generally, this meant that I did my best to protect the pupils and teachers from any (physical or non-physical) harm that may occur as a consequence of my research. This includes treating their information with confidentiality, storing the field notes and recordings on a safe, university-approved and -protected server, where only I have access to them, using pseudonyms for all teachers and pupils, as well as the class and the school. I have omitted or changed some details about the participants in order to safeguard their anonymity. As I described at the start of this chapter, the principle of 'do no harm' also meant, in my case, intervening when I thought physical harm was potentially about to happen - which, to my relief, was necessary only once, in the moment described in the third opening vignette at the start of this chapter.

While being at school, I was always open and honest about my work. On the first day of fieldwork, I explained to the pupils that I was a researcher from Maastricht University, and that I was going to spend a long time with them to do research on language and culture. This was, admittedly, a vague description of my topic, but at this early stage I did not have very defined ideas about what I would focus on. This succinct explanation seemed more than enough to the pupils, who seemed to have no clear grasp of (or much interest in) the exact research that I was there to do. All teachers at the vmbo-section of the school were informed of my presence by the Dean, who had enabled my access to the school. Every time I met a new teacher of class 3/4b, I introduced myself, explained my reason for being there, and asked for permission to record in class.

Despite my openness, pupils remained uncertain of my role (as illustrated at the start of this chapter, in the first two vignettes). It is often the case with

${ }^{15}$ Case code ERCIC_001_04_01_2017. 
ethnographic research that informants make sense of the ethnographer in terms of the roles that they already know, and in schools, adults are usually interns, teachers, or some other kind of authority. For a long time, the pupils of class $3 / 4 \mathrm{~b}$ thought $\mathrm{I}$ had one of those roles and it took them a while to trust that I was not going to discipline them or tell the teachers if they broke any school rule. At the start, they also thought that my 'research about language' was about the 'quality' of their language, or their swearing. Whenever one of them cursed, another pupil would immediately tell me: "Write that down, miss!" (e.g. Field notes, 27 January 2017). Some other pupils thought my research was about the people they referred to as buitenlanders 'foreigners' (Field notes, 12 December 2017). Each time that something like this came up, I explained that my research was about language and culture, but that culture included everyone, not just 'foreigners.' Understandably, the pupils found 'language and culture' a very vague topic. They were not familiar with the concept of a $\mathrm{PhD}$, and having a job where you just sit in class doing what to them seemed nothing was puzzling to them. As time passed, however, they did not seem to care very much about what exactly I was doing there, accepted my presence, and engaged with me rather freely.

Few teachers were familiar with the concept of a $\mathrm{PhD}$, and like pupils, initially they often saw me as a student or intern. Many thought (or maybe hoped) that I would focus on how they could help their pupils 'improve' their language, or how they could stop pupils from swearing. At the start, some teachers seemed to orient to me as an authority or expert on pedagogical matters. They apologized to me for a 'chaotic' class (Field notes, 25 January 2017); or asked me how I thought their class had gone (Field notes, 17 February 2017). I was always quick to explain that I had no expertise on pedagogy or teaching, and that those things were not part of my research. My rectifications sometimes seemed to leave them struggling to understand the added value of research on 'language, identity, and culture.' As time progressed, teachers became used to my presence to such an extent that it happened a few times that they would hand me a test as if I were just another pupil. Furthermore, I had started to have more focus in my research and it became easier to explain what I was working on in more concrete terms.

I obtained informed consent from the participants in several ways. I explained my research to the pupils on the first day at South High School, and gave them the opportunity to ask questions and to approach me if they wanted to be excluded from the research, and told them that they could do this at any point during the fieldwork. I explained that I was going to make audiorecordings, which only I was going to have access to, and that they would remain anonymous in any publications. Since they were minors, I furthermore gave them a copy of a letter for their parents or caretakers, in which I explained 
the research and gave them the opportunity to exclude their child from the study. This letter was also made available through the digital platform through which the school communicated with parents and caretakers. The parents of one pupil withdrew their child from the study. This pupil is thus excluded from any of the analyses presented in this dissertation.

The results of my research - that is, all publications - will be made accessible to the school, teachers, and to the extent that it is possible, the pupils themselves. The raw data (field notes and audio recordings, and some photos) are stored on a safe, university-approved and -protected server where only I can access them in order to ensure the participants' anonymity.

Although it is not explicitly mentioned in the latest (2012) version of the Principles of Professional Responsibility of the American Anthropological Association, another important ethical matter in ethnographic fieldwork is reciprocity (Robben and Sluka 2012; see Berreman 2012 for a critique of the changes in the PPR). The 2009 version of the Principles states: "[Anthropologists] should recognize their debt to the societies in which they work and their obligation to reciprocate with people studied in appropriate ways" (American Anthropological Association 2009). After consulting with the school board, it was decided that I would come back to school after the fieldwork to give a presentation about my findings to interested teachers. I did so in December 2018, during a weekly teacher's meeting. As for the pupils, they have (jokingly) demanded cash for their participation. I hope that they will consider my attempt at their fair representation throughout this dissertation, my gratitude, and the chocolates that I brought them on my final day, an acceptable alternative.

\subsubsection{Reflexivity}

Ideally, reflexivity does not reside in a sub-section of a subsection of a methods chapter. It should be part of an ethnographer's entire practice from the moment that fieldwork commences until (and after) it is written down. Reflexivity, i.e. "the conscious self-examination of the ethnographer's interpretive presuppositions" (Robben 2012, 514) should be part of data collection, analysis, and transmission at all stages. I have intended to be reflexive throughout my fieldwork, and in the writing of this dissertation I have aimed to acknowledge my own role in "shaping the object/subject studied" (Borneman and Hammoudi 2009, 2). I was often part of the interactions that I analyze, and those interactions would have happened differently, or in some cases would not have happened at all, had I not been there. Each analyzed interaction throughout the dissertation therefore includes a short description of my role in it, even if it was only as a bystander 
or eavesdropper. In this section, I will highlight some other important aspects of how my positionality and presuppositions may have influenced data collection and analysis, and how I dealt with that during fieldwork, analysis, and writing.

The first and foremost issue about how my positionality shaped my findings is the simple fact that I was an adult researcher trying to learn about adolescent pupils. I always tried to mitigate any power differences, for example by sitting with the pupils in class, chatting and joking with them in an informal style, and 'not noticing' when they broke school rules (or sometimes participating in breaking minor rules, for example by looking at a pupil's phone with them). I tried to develop relationships with the pupils on an informal basis, the way I might with acquaintances. They sometimes tested me, for example by teaching me a cuss word in Arabic and telling me it had another (innocent) meaning - to which I reciprocated with the kind of banter they also engaged in (i.e. by jokingly using those words to refer to them). Despite these efforts, I had an undeniable position of authority in comparison to the pupils. I did not do schoolwork or receive grades, did not depend on receiving a diploma, and teachers approached me more as a (different kind of) colleague than as another pupil. When I engaged in informal conversation with pupils, teachers would not discipline me (or only jokingly), but they did discipline pupils when they chatted among each other. This hierarchical ordering was also reflected in how pupils addressed me: although I had asked them to call me by my first name, many addressed me as juf, ('teacher' or 'miss'), and often used the formal second-person singular pronoun $u$ instead of the more informal $j i j$ or $j e$ (you). At the same time, several pupils mentioned that they saw me as 'another pupil, but different' or as 'a kind of friend' (Field notes, 13 March 2018).

Aside from my age and position as a researcher, my gender also influenced my data collection. As mentioned earlier, I preferred participating in Care and Well-being classes, where the majority of pupils were female, over the all-male environment of Mobility and Transport. I felt that my gender made my participation in Care and Well-being easier, and that it facilitated the establishment of rapport with the (mostly female) pupils in this specialization (and that it made it more difficult to establish rapport with the male pupils in Mobility and Transport). Aside from being female, I am university-educated, middle-class, white, not religious, from the center-east of the Netherlands, and categorizable as Nederlander according to the pupils' categorization logic - i.e. without known or recent migration background. I found those last two points to be especially significant since a large part of my analyses concerns the pupils' practice of categorization on the basis of having (or not having) a migration background. At the start of my fieldwork, and a 
couple of times later on, pupils asked me 'what descent' I had (“Wat voor afkomst heeft $u$ ?", Field notes 17 February 2017). This implies that this was relevant information to them. My reply that my descent is Dutch meant that I was a categorical 'Other' to some pupils, and that they oriented to me as an outsider in certain matters related to language, culture or religion. I was also an outsider to many of the pupils that were also categorized as 'Dutch,' however. I am not from Limburg, and I am therefore seen as 'Hollander' by many people in Limburg. This is hearable in the way I speak Dutch, which may have influenced how pupils as well as teachers saw me, since a centralDutch accent in Dutch like mine has been found to be regarded as more prestigious than a Limburgian accent (Grondelaers, van Hout, and Steegs 2010). Aside from being different from some pupils in terms of where we grew up or our migration backgrounds, I found that I was an outsider in many more ways: for instance, I have a background in pre-university and university education whereas the pupils followed the vocational track, and, as a researcher, I had a peculiar role in the school that no one else had. It is difficult to surmise the exact consequences of all these aspects of my positionality, but it is likely that they influenced the way pupils (and teachers) saw me. In a way, the convergence of all those axes of 'outsider-ness' seemed to diminish the importance of each single one of them. As a non-member of so many categories they associated with, I could get away with being an incompetent participant in this particular context. It enabled me to take on the role of "professional stranger" (Agar 1996), that is, an intentionally incompetent, but curious stranger, who asked them about many different aspects of their lives.

At the same time, my interest in the topic of categorization did create a number of dilemmas, some of them in terms of practices in the field, and others during the phase of 'writing up.' Firstly, in terms of practices in the field, I wanted to know more about the categories that pupils used - most prominently Marokkaan, Turk, buitenlander and Nederlander - but I felt that using those terms myself could have a strong othering effect, especially as an outsider to their communicative practices and as - in their eyes - a Nederlander. I did not want to give the impression that I saw pupils with migration backgrounds as somehow essentially different from pupils without migration backgrounds, thereby reproducing a categorization system that is the topic of so much sociopolitical debate and, more importantly, that lies at the root of so much stigmatization and inequality. The (admittedly imperfect) way I tried to tackle this was by listening closely when pupils themselves would bring up the categories in conversation, and by trying to avoid using these terms myself. There were moments where I did want to ask about the categories, however, and in those interactions my discomfort is evident from 


\section{Nederlanders and buitenlanders}

my hesitations, avoidance of terms, and false starts. Take, for instance, the following excerpts:

1 P Waarom mag je die mensen niet dan?

Why don't you like those people then?

(26 October 2017. Full interaction analyzed in Chapter 3)

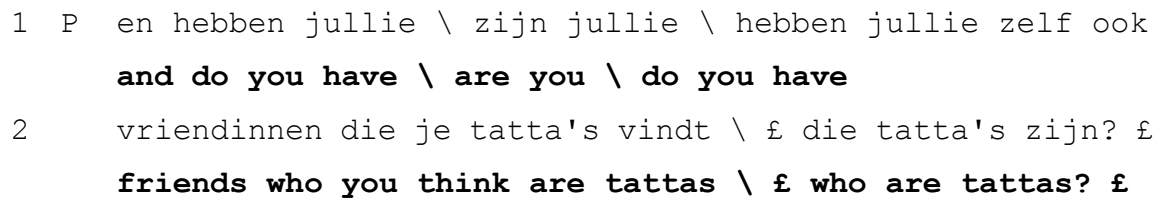

(16 June 2017. Full interaction analyzed in Chapter 4)

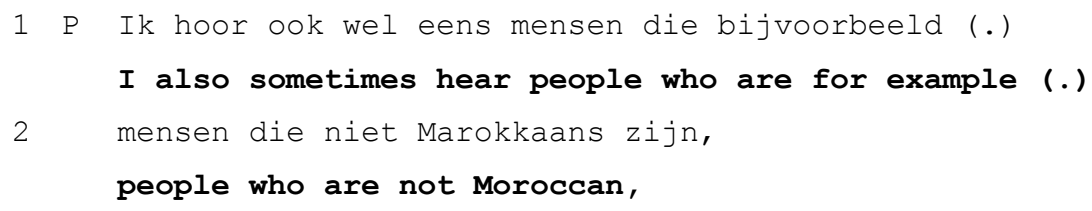

(16 June 2017. This excerpt is not part of an analysis in this dissertation)

In the first example, I avoid a category label that a pupil had just used ('Hollanders') and instead ask about 'those people.' The second example contains false starts and self-repair. I first say 'friends who you think are tatta's,' then realized that to them, this was not a matter of opinion, and changed to 'who are tatta's' in smile voice that suggests discomfort. The third example shows that I was even more uncomfortable using a term such as Marokkaan, and again I use the construction "people/friends who are" instead of using their term. In all situations, I felt caught between the idea of asking so-called 'native-language questions' promoted by (some) ethnographers, i.e. using the terms and phrases commonly used by the informants, to 'minimize misunderstandings and display cultural understanding' (e.g. Spradley 1979) on the one hand, and on the other hand, wanting to avoid giving the impression that I subscribed to that categorization system, and thereby reproducing it. At the same time, avoidance of this topic and of ethnic category labels in general seem not to be a solution either (e.g. Pollock 2004; Seeberg 2003).

On a more substantial level, by focusing my analyses on descent and ethnic boundaries, I run the risk of contributing to the reification of those categories and thus being complicit in the "fetishization of ethnicity" (Rampton 2005, 7) that I try to overcome. As Seeberg (2003, 29) writes: 
"Every time I single out ethnic identity as my object of study, through the descriptive use of ethnic labels, I also contribute to the idea that ethnic identity is important." ${ }^{16}$ I have also asked myself the questions that Seeberg $(2003,30)$ asks:

How do I make visible differences that are relevant to people in their daily lives as well as to my analysis, without trespassing, without presupposing the validity of some categories at the expense of others, and without contributing to racist ideas?

Considering my own positionality as someone without a (known) migration background, analyses of social categorization in ethnic terms might be seen as being, or contributing to, exoticism or orientalism (Said 1978; see also C. King 1977). If I had had a migration background myself, I might not have been surprised by categorizations with the terms buitenlander, Marokkaan, Turk, and Nederlander to the same extent. My selection of phenomenon to study thus most certainly stems from my own positionality. I hope that in my analyses of that phenomenon, however, the attention that I give to describing and analyzing practices by which boundaries were constructed and negotiated, and by avoiding descriptions of the 'cultural stuff that is supposedly enclosed by those boundaries (Barth 1969), I am able to show that the pupils who mostly engaged in categorization (i.e. those with migration backgrounds) were not different, strange or 'exotic' for doing so, but that they engaged in social practices that adolescents have been described to engage in in many different contexts and times (e.g. Androutsopoulos and Georgakopoulou 2003; Bucholtz 2011; Eckert 1989). In other words, I hope that a close reading of this dissertation might 'de-exoticize' these pupils' practices of categorization by showing that the social processes underlying them are very similar to those found in other (adolescent or other) communities across contexts and time periods, and that one reason why these pupils did this with ethnic terms specifically can be found in present day debates and discourses in Dutch society.

\subsection{Conclusion}

In this chapter, I described how I went about answering the dissertation's research question: What do ethnic categories and linguistic resources mean, and do, for the pupils and teachers of South High School? I first sketched an

${ }^{16}$ For reflections on a similar dilemma with regard to gender categories, see Dietze et al. (2019). 
overview of the larger contexts in which the study took place, reviewing, in particular, the Dutch educational context, immigration discourses in the Netherlands, and the characteristics of the city of Venlo, and the province Limburg, where this study was conducted. Some background knowledge of those contexts is necessary to situate the findings of the chapters to follow. Having explained the theoretical underpinnings of ethnography in Chapter 1, in the current chapter I described how I implemented those principles in the context of my fieldwork with class 3/4b of South High School. I spent a total of nine months with one class, in their third and fourth years of secondary school, observing and participating in the pupils daily lives at school. In keeping with the ethnomethodological and conversation analytic principles explicated in Chapter 1, I paid close attention to the interactional practices of the participants, and sometimes participated in these interactions, trying not to be of major influence on the topics of their conversations. In the final section of this chapter I discussed the ethical considerations that guided the process of conducting ethnographic fieldwork and writing the analyses, and I reflected on how my own presence may have influenced the context, participants' behavior, and how my own background may have influenced the analyses presented in the chapters to follow. 


\section{Negotiating the characteristics of Turken, Marokkanen, buitenlanders, and Nederlanders"}

Field notes, 20 December 2016

On a chilly Tuesday morning in December 2016, I visited South High School for the first time. I parked my car in the driveway, announced my presence at the reception and waited for the Dean to pick me up. Mr. Hendriks and I had had surprisingly brief contact through email: I wrote to him introducing myself and my research ideas, and in his reply, he proposed a day and time for me to come by the school. One week after that email, I found myselffollowing him through the mazelike hallways of the school building, having flashbacks to my own high school times. We sat down in his office, and while he asked me about my interest in 'cultures' and 'languages,' Mr. Hendriks closely monitored the pupils outside in the hall through the tall windows on each side of the door.

I expected to have to convince him to allow me to do fieldwork at his school, but the Dean quickly agreed with it all. What's more: he had already thought of a class for me to do my research with. "Class $3 b$ is a nice group, quite diverse, and they are not so disorderly, so the teachers will probably also be fine with having you join them in class." He then sat down behind his computer to illustrate what he meant by 'diverse.' Scrolling through the list of names of the pupils in class 3b, Mr. Hendriks told me something about each of them. "Dounia is Moroccan, she works hard but she sometimes has difficulties keeping up. Yildiz is Turkish, she is smart, but she should work harder. Dennis is one of the most intelligent ones even though he has severe dyslexia. Oh, and here we have Jay, he's from the Caribbean."

\subsection{Introduction}

The above vignette illustrates the first time my attention was drawn to the tendency to categorize people in terms of ethnicity or descent at South High School. When I started my fieldwork with class $3 / 4 \mathrm{~b}$ about three weeks after the first meeting with Mr. Hendriks, described in the vignette, I found out that it was not surprising that he had been able to enumerate every pupils'

"Parts of this chapter will appear in van de Weerd, Pomme. Forthcoming. "Ethnic labeling among pupils with migration backgrounds: 'Turks', 'Moroccans', and 'foreigners' in the Netherlands." Dutch Journal of Applied Linguistics. 
national origins. Descent turned out to be a recurrent topic in the pupils' daily discourse: they referred to themselves and others as Marokkaan 'Moroccan,' Turk 'Turk,' buitenlander 'foreigner,' and Nederlander, Hollander or tatta 'Dutch person' many times a day. Sometimes they did so in conversations where it sounded relevant to me as a newcomer to their interactional customs, for example, when they chatted about the combination of cultural traditions they had observed during a wedding between a man with Turkish background and a woman with Moroccan background. At other times I struggled to understand the relevance of someone's (perceived) descent. Why would you greet a friend by saying "Hi Turku"? I also wondered why they referred to themselves as buitenlanders 'foreigners' if they had been born and raised in the Netherlands. These initial questions turned into a sustained attention to how pupils used ethnic labels throughout the nine months I spent at South High School. In this chapter, I explore how pupils in class $3 / 4 \mathrm{~b}$ constructed, and negotiated the meanings of, the categories Marokkaan, Turk, buitenlander and Nederlander. Although all pupils seemed to agree on the existence of categories with these labels, the implications of membership in those categories were constantly negotiated and debated.

The analyses in this chapter confirm previous research that has found that ethnic category labels can index dimensions of social differentiation other than ethnicity or affiliation with a country or nation (e.g. Chun 2011; Nørreby 2019; Ortner 1998) and explores how this is done in the particular context of class 3/4b. This serves to build a base understanding of how pupils established and gave meaning to categories, which will enable the reader to better contextualize the contents of the following chapters.

The remainder of this chapter is organized as follows. In section 3.2, I provide a short overview of literature that explores the complex meaning potential of ethnic category labels. Section 3.3 contains the data analysis. Pupils of class 3/4b often related the categories Turk, Marokkaan, buitenlander and Nederlander to characteristics such as physical appearance, religion, or clothing styles. In those discussions, pupils negotiated the relation between those characteristics and category membership: the category-incumbent features that pupils constructed as central to category membership differed from one to the next moment, and also shifted between pupils. In the subsections of 3.3, I analyze negotiations of category membership in relation to (un)belonging, religion, physical appearance, language and clothing styles, and behavior. 
Negotiating the characteristics of Turken, Marokkanen, buitenlanders, and Nederlanders

\subsection{The meaning potential of ethnic categories}

In many different contexts, it has been observed that ethnic category terminology is part of multifaceted practices of identification and has a complex meaning potential. In a study in a California junior high school, for example, Lee (2009) found that pupils labelled each other 'Mexican' if they were of Latin-American descent, even when their migration background was known to be in a country other than Mexico. As Lee $(2009,40)$ observes, "[r]ather than using predetermined demographic categories, youth adopt their own categories to label ethnicity." Nørreby (2016) also shows that predetermined categories and 'old binaries' such as majority/minority do not account for the experience of young people in contemporary urban environments. He describes the case of a young man born and raised in Copenhagen with a migration background in Palestine, who sometimes referred to himself as 'Dane' and at other times as 'Palestinian,' "constantly recontextualiz[ing] the different ethnic categories he employ[ed]" (Nørreby 2016, 217-18). Among a group of friends observed by Shenk (2007, 199), "Spanish linguistic proficiency, place of birth, and purity of bloodline" functioned as criteria to determine 'Mexicanness.' Several authors have noted that issues of authenticity and authentication play an important role in categorizing self and others: Young men of Surinamese descent in Rotterdam, for example, constantly redefined categories such as 'Surinamese' and 'Dutch' and thereby positioned some individuals as authentic and others as inauthentic (Cornips and de Rooij 2013). Møller (2017) pays attention to the 'polycentric norms' that organized rights and affordances to use terms related to ethnicity in a Copenhagen school class, where "ethnicity [was] not strictly organized in fixed positions, but instead [became] a dynamic resource in practices of languaging as well as processes of identification” (Møller 2017, 142). Racial or ethnic labels can also seep into other domains of social differentiation, and can be linked to e.g. dress or music styles. Bucholtz (2011), for example, describes how race as a social boundary was so salient that it influenced how other boundaries were drawn. In the high school she studied, "stylistic categories were racially defined to such an extent that teenagers who adopted a style that did not conform to their own racial assignment ran the risk of being derided as 'wannabes' or accused of 'acting black' or 'acting white" (Bucholtz 2011, 43).

While this and much other work has concentrated on how ethnic and racial labels are implicated in youthful (meta-)discourses of ethnic or racial identity, others have drawn attention to how people may use such labels to negotiate and construct other kinds of identities. Chun $(2011,404)$ notes that 
ethnic or racial terms can be used to provide ideological commentary on racial authenticity, but also "on gender and class performance.' Also Nørreby (2019, 138) describes how ethnic labels can be "locally reconfigured to do non-ethnic work" such as, in his case, negotiating local social status hierarchies.

In sum, ethnic or racial labels have a complex meaning potential that can differ widely from one to the next context: they can be a part of complex ethnic or racial identity work, but they can also be adopted to negotiate other social boundaries, such as class, gender, or cultural styles. In the next sections, I explore the wide array of associations that ethnic labels carried in class $3 / 4 \mathrm{~b}$.

\subsection{Marokkanen, Turken, buitenlanders, and Nederlanders and debates about locally associated characteristics}

Figure 3 contains an overview of the categories and sub-categories that the pupils of class $3 / 4 \mathrm{~b}$ used most often in my presence.

Figure 3: Categories and their segmentation observed in class 3/4b

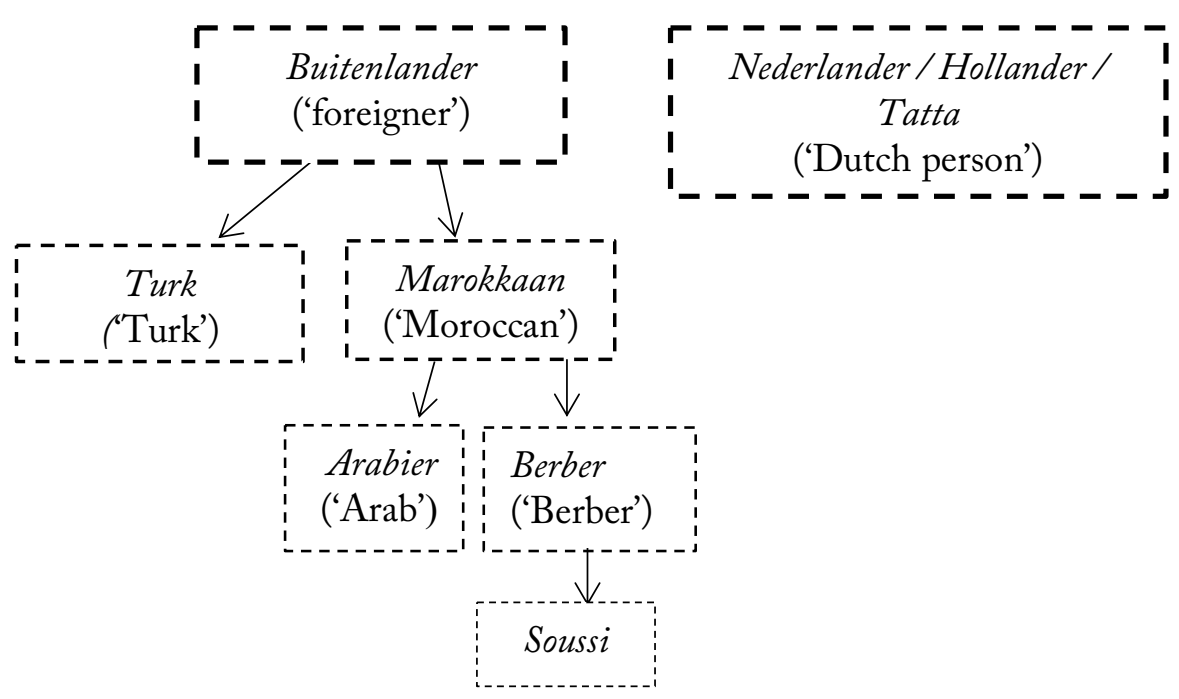

This figure may seem to suggest that those categories were like boxes where it was always clear who fit inside and who did not. Although pupils all seemed to agree that these categories existed, however, this chapter will show that they also constantly negotiated and debated who fit in which category, what it meant to be a member of that category, and how to deal with people or behaviors that did not conform to expectations about categories. Figure 3 
serves as a clarification of the labels that pupils of $3 / 4 \mathrm{~b}$ used, and the way they were usually organized, but not as a schematic overview that always functioned in a straightforward manner.

The pupils usually treated the labels depicted next to each other in Figure 3 as mutually exclusive: being buitenlander meant that you were not Nederlander, and being a Turk was treated as categorically distinct from being a Marokkaan. In certain occasions, the pupils sub-segmented the category Marokkaan into Arabieren 'Arabs' and Berbers 'Berbers' (as reflected in the figure by the positioning of the boxes underneath each other) (cf. Baumann and Gingrich 2004). The category Soussi (a Berber people of Southern Morocco) was mentioned only a handful of times, for example between Amira and Dounia who both categorized themselves as Berber, but Dounia was Soussi and Amira was not. The use of these different labels in different contexts did not form a contradiction to the pupils but merely represented various levels of specificity. Selection of one over the other depended on the interactional context and interlocutors. On the one hand, pupils all used this same set of terms to refer to themselves and other people, and they seemed to agree that there were such membership categories which were self-evident and straightforward and could 'objectively' be identified in the outside world. On the other hand, the interactions in this chapter show that pupils constantly negotiated the meaning of category membership, debated which characteristics were essential and which were marginal or variable, and sometimes showed that they might as well be seen to belong to a different category. While they were categorizing, they were thus also 'particularizing' (Billig 1996): they treated people as members of categories in some instances, and as exceptions, or simply as individuals, in many others. That said, I will now sketch an overview of some characteristics that pupils often mentioned when negotiating the meanings of categories.

Pupils used the label buitenlander 'foreigner' to refer to all people perceived as having origins in countries other than the Netherlands - also when these people themselves had been born in the Netherlands (which was true for all except two pupils in class $3 / 4 b$ ). It functioned as an umbrella term. The pupils' seemingly 'neutral' use of this term is striking, as buitenlander is often seen as a term that stigmatizes and discriminates. In the late $80 \mathrm{~s}$ and early 90s, the term buitenlander was replaced in Dutch academic, political and popular discourse, by the word allochtoon (see Chapter 1), which, in turn, was replaced by 'Dutch person with a migration background' in 2016 (Schravesande 2016). In class 3/4b, I only witnessed one pupil use the term allochtoon a few times. 'Person with a migration background' was not used by the pupils. After a societal studies class in which the teacher had mentioned 
that the term 'foreigner' was perceived as stigmatizing, I asked some pupils about their opinion on this, to which one of them succinctly stated: "That's what we are, isn't it?" (Field notes, 16 June 2017).

The most frequently used sub-categories of buitenlander in this context were Turken and Marokkanen. Pupils used these labels to designate people with a migration background in Turkey and Morocco, respectively. This second level of segmentation in Figure 3 only consists of these two categories, not because these were the only kinds of buitenlander 'foreigner' imaginable in this context, but because they were the only sub-levels about which I commonly heard the pupils of class $3 / 4 \mathrm{~b}$ speak. Not all pupils categorized as buitenlander could be categorized as Turk or Marokkaan, but I did not observe any of the four pupils of class 3/4b with migration backgrounds in countries other than Turkey or Morocco employ labels for themselves in lower levels of segmentation than buitenlander. For example, I never heard Ahmed, whose parents came from Afghanistan, label himself as Afghaan 'Afghan.' For the same reason, Figure 3 lacks sub-categories under the category Turk (for example, I did not hear pupils sub-segment themselves or others as Kurds).

The labels buitenlander, Turk and Marokkaan were mostly used by pupils who categorized themselves as member of one of those categories, that is, pupils with migration backgrounds. These pupils did not refer to themselves as Nederlander 'Dutch person,' but they did sometimes say they were Nederlands (adjectival 'Dutch'). They used the label Nederlander for people without (known or recent) migration backgrounds, and Hollander and tatta functioned as its synonyms. This particular use of Hollander is striking since in the province of Limburg - in which the school is located - Hollander is commonly used to refer to a Dutch person from outside Limburg. In class $3 / 4 b$, however, Hollander was used in the way it is used outside Limburg, namely to refer to people imagined to be somehow prototypically Dutch. This included people who could be labelled Limburger. Tatta, the other synonym for Nederlander or Hollander that pupils used, comes from ptata or tata ('potato' in the Surinamese language Sranan Tongo) and refers to a stereotypical image of a (white, potato-eating) Dutch person (Cornips and de Rooij 2013). There is thus a connotation of colonial relations, but none of the pupils in this study had migratory relations with Surinam and they did not show awareness of the origins of this term. Pupils' use of the terms tatta and Hollander shows that categorization was not a purely local practice, but that it was influenced by categorization practices on a larger scale in the Netherlands. It points to the circulation of discourse surrounding those categories, for instance on the internet (for example, there is a popular "meme" genre called 'tatta's be like,' which revolves around humorous images of prototypical "Dutch" habits, see Muller 2014). 
Negotiating the characteristics of Turken, Marokkanen, buitenlanders, and Nederlanders 73

Pupils who were referred to as Nederlander did not often categorize themselves or others. Being Nederlander seemed unmarked to them. Moreover, this category lacked prestige: Pupils with migration backgrounds often referred to it with rather negative characteristics such as being stingy or boring. Potentially due to those negative associations, pupils categorized as Nederlander rarely used this label for themselves. They also did not use the labels 'Turk,' 'Moroccan' or 'foreigner' often, especially not in the presence of pupils who categorized themselves as such. Labelling others 'Turk,' 'Moroccan' or 'foreigner' while not being categorized as such oneself could result in social sanctioning due to associations with stereotyping or discrimination.

The pupils of class $3 / 4 \mathrm{~b}$ spent much time discussing and debating the characteristics that defined category membership. The following sub-sections give an overview of topics in relation to which pupils often mentioned categories. In the first of these (3.3.1), I discuss the topic of national unbelonging. Pupils did not often talk about issues of 'belonging' or 'feeling at home' in the Netherlands, however. I have nevertheless included it because of the frequency with which the use of these labels for self-categorization are understood to be related to issues of belonging in popular as well as academic discourse. Analysis of some interactions with and between these pupils serve to complicate that often presupposed relation. The other sub-sections (3.3.2 to 3.3.5) illustrate the characteristics that the pupils themselves discussed in relation to categories. Each section shows how the categories Nederlander, buitenlander, Turk and Marokkaan were part of "presumed common-sense knowledge of social structures' which members [...] oriented to in the conduct of their everyday affairs" (Hester and Eglin 1997, 3), but also how the 'knowledge' associated with categories was often contested and negotiated.

\subsubsection{Categorization and debates about national (un)belonging}

As mentioned in the introduction to this chapter, use of labels such as Turk, Marokkaan and buitenlander for self-reference by people with migration backgrounds is often interpreted as an expression of feelings of belonging to the countries those labels refer to, or a lack of belonging to, or integration in, the Netherlands. My conversations with pupils who referred to themselves with those labels suggest, however, that those interpretations overlook important nuances and may not always be accurate. For example, when I spoke to pupils who categorized themselves as Marokkaan or Turk about Morocco or Turkey, they would be quick to signal that they enjoyed holidays there, but that they would not want to live there, as it was 'too different' (e.g. field notes, 16 June 2017). Aligning with labels thus did not stand in the way of 


\section{Nederlanders and buitenlanders}

disaligning with the countries to which they refer. This is also illustrated by Example 3.1, which shows how two pupils, who regularly categorized themselves as Marokkaan and Turk, respectively, constructed a different category for themselves than for the people in those countries, who they referred to as 'strange.' The interaction occurred when I sat down during class with Dounia (who often categorized herself as Marokkaan) and Yildiz (who often categorized herself as Turk), who were working on an assignment together. The teacher was on the other side of the classroom, and the two (who were good friends) engaged in informal conversation. They had just been discussing the city in Morocco where Dounia's family is from when I asked Dounia the question in line 1.

Example 3.1. 15 May 2017. Participants: Dounia (D), Yildiz (Y), and the researcher (Pomme, $\mathrm{P}$ in the transcript).

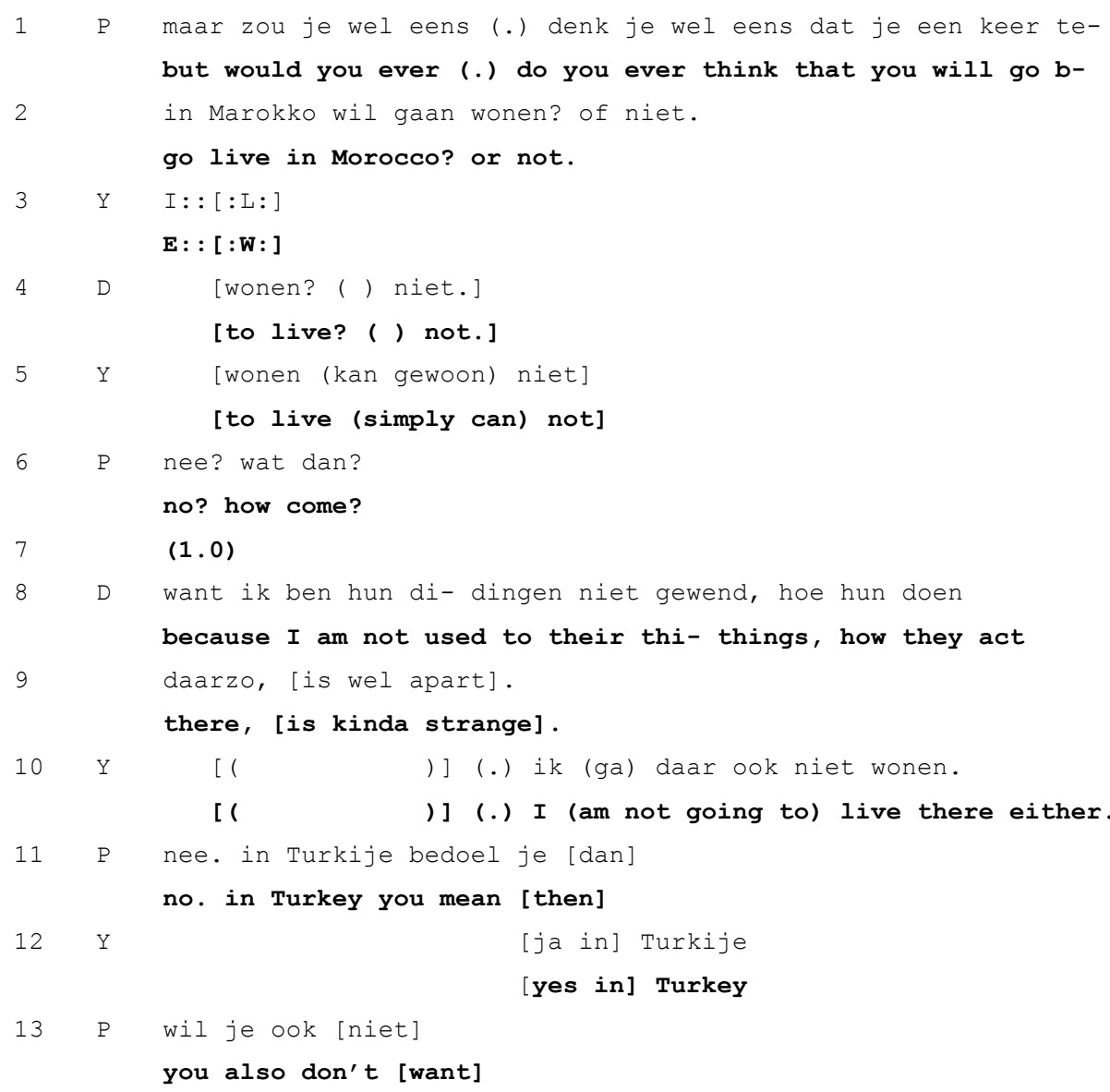


Negotiating the characteristics of Turken, Marokkanen, buitenlanders, and Nederlanders 75

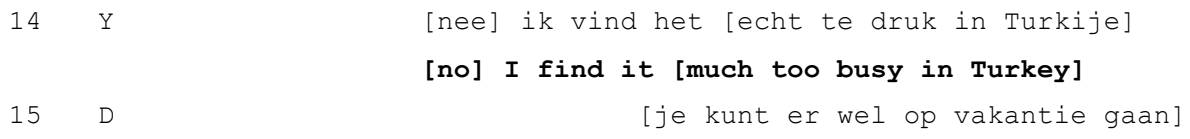

When I ask Dounia whether she would consider living in Morocco in lines 12, Yildiz is the first to react. Her exclamation in line 3 ('Ew!') signals shock, surprise, potentially even disgust. Her reaction does not seem to address Morocco specifically, though, which could have been interpretable by Dounia as an insult. Dounia almost immediately aligns with Yildiz. Although their exact words in lines 4-5 are hard to understand because they are speaking simultaneously, both girls make clear that they would not consider living 'there.' 'Living' is heard in opposition to going for holidays, as made explicit by Dounia in line 15. 'There' constructs an opposition with 'here in the Netherlands.' In lines 8-9, when she says 'how they act there,' Dounia implicitly constructs a 'we here' and frames 'Moroccans' in Morocco as categorically different. She constructs two kinds of Marokkaan: a kind of "diasporicallyMoroccan" (Wagner 2017) like herself and a 'locally-Moroccan' (who she says are 'kinda strange,' line 9) (cf. Wagner 2018).

In line 10, Yildiz says she does not want to 'live there either.' Notably, however, Yildiz labelled herself 'Turk,' and we had been talking about Morocco. Dounia's use of the rather general words they and there, in lines 89, seem to have made the link to Morocco, specifically, irrelevant to Yildiz. She unproblematically reproduces the term there and uses it to refer Turkey. There has become a concept that means 'not here in the Netherlands' whether that is Morocco or Turkey - and they can both relate to it in similar ways. Clearly, identification with the label does not have to entail a great sense of connection to the country.

Conversations like this, in which Morocco or Turkey were constructed as the place of the Other, occurred regularly: on one occasion, I overheard a number of pupils laughing about their shared experience of wearing ripped jeans - which were fashionable during that time in the Netherlands - during their summer holidays in Morocco and Turkey. Together, they laughed about having been looked at with pity as people had apparently suggested they had been unable to afford better jeans. "Bro, my jeans are more expensive than your entire outfit!" one of the girls replied in hypothetical hindsight (field notes, 16 June 2017). 


\section{Nederlanders and buitenlanders}

The connection between labelling oneself Marokkaan, Turk, or buitenlander on the one hand, and having feelings of belonging to Morocco or Turkey, or (un)belonging to the Netherlands on the other, was thus debatable in the case of many pupils. Pupils with migration backgrounds regularly talked about the Netherlands as their own country and Morocco or Turkey as their parents' country. At the same time, the term Nederlander was not part of their discourses of self. Among these pupils, Nederlander was associated with many category-incumbent features that they did not apply to themselves. They often linked this category to not-so-desirable traits such as being boring, stingy, or following parents' rules. At the same time, they were aware that Nederlander was also an administrative or 'official' category, and that, in that sense, it could apply to them. In the early stages of my fieldwork, Amine declared to me that he was Marokkaan, and when I asked whether he was also Dutch (using the adjectival form 'Nederlands'), he replied, "Well yes, I speak Dutch, don't I?" (Field notes, 21 March 2017). In the field note in Example 3.2, Amine also negotiates what it means to be a Nederlander (in this case using the words tatta and Hollander), this time in conversation with Meryem and me.

\section{Example 3.2. Field notes, 29 March 2017.}

I am sitting with Meryem and Amine, who are working on a book assignment in relation to their Care and Well-being cooking classes. The assignment mentions something about 'andijviestamppot' (a dish of mashed potatoes with endive), and Amine comments that it's a typical dish for tattas. "Do you know what that is?" he asks me, in reference to the word 'tatta.' First, I say I do, but then I decide I want to hear his explanation and ask what it means. "Hollanders," he replies. "But aren't you a Hollander?" I ask him. "No!" he says with conviction. Then Meryem joins into the conversation: "But you have a Dutch passport, right." She smiles while she says this, her question comes across as a sort of challenge. Amine: "Yes." I say to Meryem: "You do too, right Meryem?" - "Yes." Then, she turns to Amine and, while laughing, does a fist bump with him: "We are tattas!"

Amine and Meryem usually referred to themselves as, respectively, Marokkaan and Turk. As mentioned earlier, pupils who categorized themselves as Marokkaan would not normally use the category Nederlander (or Hollander or tatta) for themselves (although, as the previously mentioned example of Amine illustrates, they would sometimes use the adjectival Nederlands to describe themselves). Calling themselves tatta's was the opposite of what they did in their daily labelling practices, and Meryem's laughter when she said that she and Amine were tattas sounded jocular and ironic (cf. Ford and Fox 2011; Haugh 2010). But even though it is ironic, Meryem's reaction shows that they acknowledge they can be categorized as tatta's according to certain definitions, 
Negotiating the characteristics of Turken, Marokkanen, buitenlanders, and Nederlanders 77

for example if it is taken as an administrative category connected to nationality or possession of a passport. The fact that Meryem questions Amine about his rejection of the label Hollander ("But you have a Dutch passport") suggests that she recognizes those inherent contradictions of their categorization practices. Tatta is not what they would usually call themselves, but when understood as an administrative category they could also be members of that category. The interaction can thus also be interpreted as showing Meryem's awareness that the characteristics that define category membership may be different or negotiable for others - for example for me, since I have just asked them about it.

In sum, there was no automatic link between the use of the labels Turk or Marokkaan for self-reference and strong personal affiliation with Turkey or Morocco, nor did this categorization appear to preclude feelings of belonging to the Netherlands. Even if Nederlander was not part of pupils' regular discourse of self, they also displayed awareness that according to other definitions, in other contexts, or in the eyes of other people, they were categorizable as Nederlander. In the next sections, I analyze some topics in relation to which pupils themselves often brought up categories, most prominently religion (3.3.2), physical appearance (3.3.3), style (3.3.4), and behavior (3.3.5).

\subsubsection{Negotiating the relation between categories and religion}

One topic of conversation that often led pupils to make categories relevant was religion. Many pupils in class 3/4b connected the labels Turk and Marokkaan with being Muslim. In Example 3.3 below, those categories are used as practical synonyms. During this interaction, I was sitting behind computer desks with Hatice and some of her friends, including Amira, Yildiz and Dounia. They were doing assignments, but mostly chatting. Hatice was talking about a party that she wanted to organize for her sixteenth birthday: she described who she wanted to invite ("Everyone, even my enemies"), how she was going to decorate the garden ("Pink and silver!") and what kind of birthday cake she wanted to serve ("Gucci themed"). While listening, I thought of stories I had heard from some other pupils about birthday parties, and specifically the consumption of alcohol at those parties, and I posed the question in line 1. 
Example 3.3. 2 June 2017. Care and Well-being. Participants: Hatice $(\mathrm{H})$, the researcher (Pomme, $\mathrm{P}$ in the transcript).

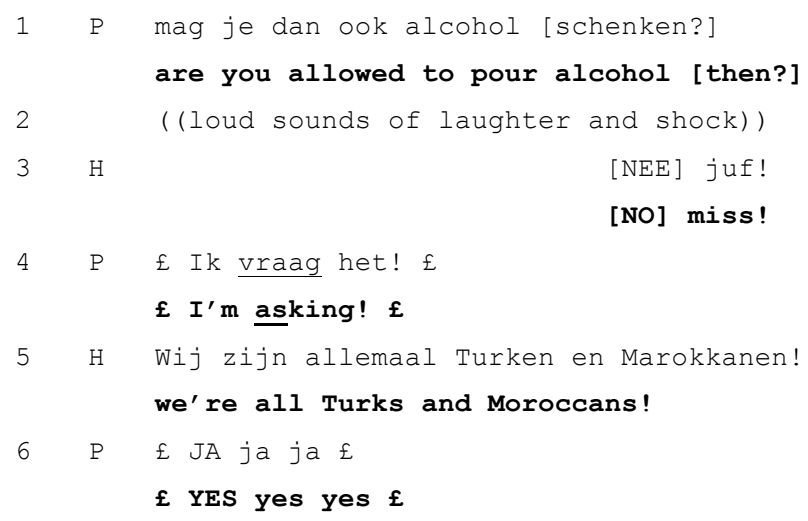

The first reactions to my question about alcohol consumption are sounds of shock and laughter, suggesting that the pupils were surprised by this question (Billig 2005). Nevertheless, Hatice's next turn is immediate and without hedges, pauses, or any other signs of her disconfirmation being dispreferred (cf. Enfield et al. 2019), suggesting that her answer is more than obvious. This is furthermore indicated by the lack of an immediate account, i.e. an explanation of why she would not pour alcohol (Pomerantz and Heritage 2013). In line 4, I can be heard as justifying myself as having 'just asked' (i.e. not having presumed anything), emphasizing the supposed innocence of my question (although I had known it could be provocative) and trying to remedy the apparent transgression of having posed this question.

In line 5, Hatice offers the account that was not immediately forthcoming in line 3. She invokes categorical knowledge of 'Turks' and 'Moroccans' to explain their abstinence from alcohol. The mention of 'Turks and Moroccans' is likely designed as an invocation of religion as a reason for not drinking alcohol, but interestingly, Hatice did not choose the category 'Muslims.' Seemingly, she expects the categories Turks' and 'Moroccans' to do the work of accounting for their abstinence more effectively than any individual or other categorical explanation (e.g. 'we do not want to drink alcohol,' 'we are minors,' or 'we are Muslims'). This suggests that the relation between these categories and not drinking alcohol was self-evident to Hatice, but since turns are always designed for a specific recipient (Sacks, Schegloff, and Jefferson 1974), it also suggests that she expects her interlocutor (me) to understand it that way. Presumably, Hatice oriented to me as member of the contrastive category Nederlanders, who must be aware of a 'common 
knowledge' that links the categories 'Turks and Moroccans' with abstaining from alcohol. In line 6, I quickly accept the explanation, signaling that I have understood and thereby backing down from my initial and somewhat strategic (see Spradley 1979) display of ignorance.

The interaction in the next example (Example 3.4) also shows how pupils sometimes used ethnic and religious categories as near-synonyms. This interaction occurred during math class, when the teacher (Mr. de Jong) joined three pupils (Meryem, Yildiz, and Amira) and engaged in informal conversation with them. I was sitting nearby but did not participate in the conversation. The interaction took place during Ramadan, and many of the pupils were fasting, including Meryem, Yildiz, and Amira. Conversations about food occurred frequently during this period, and in this case, Mr. de Jong joined one of those conversations.

Example 3.4. 19 June 2017. Maths class. Participants: Meryem (M), Amira (A), Mr. de Jong (J), Yildiz (Y)

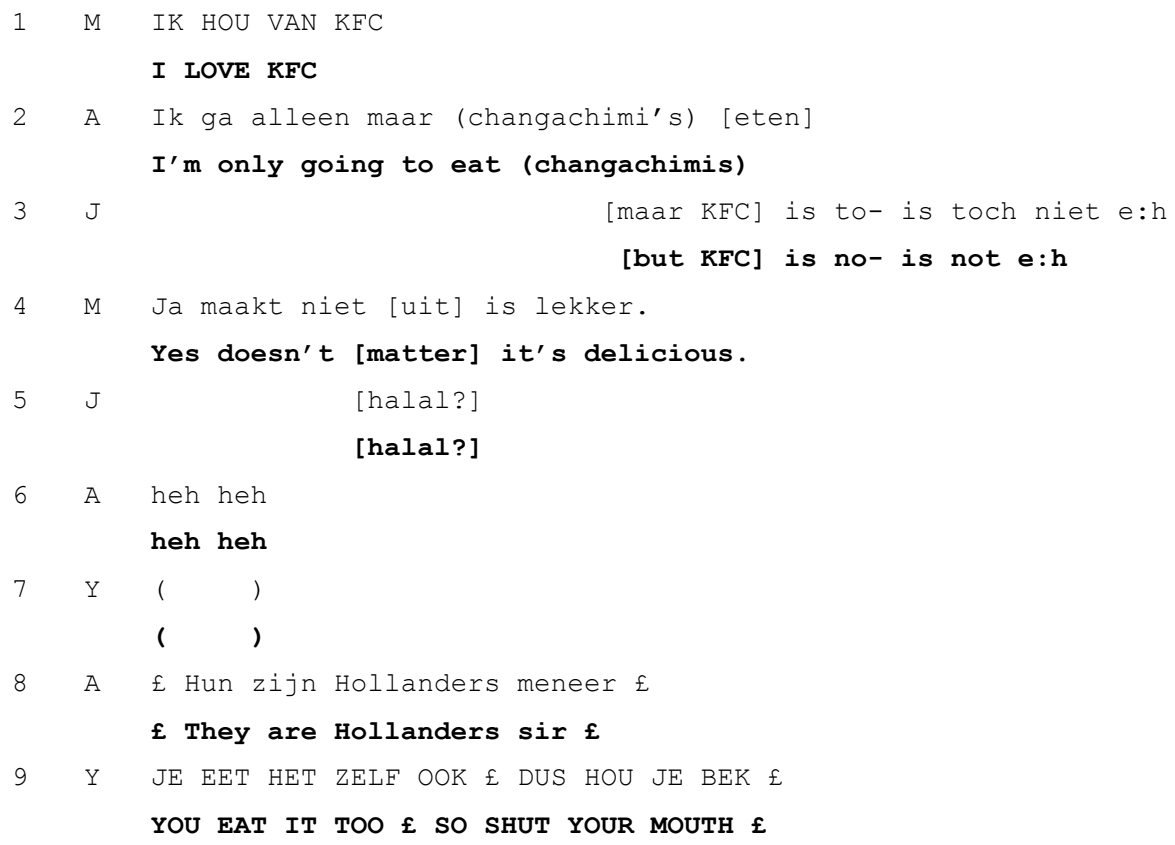

This interaction occurred while Meryem, Yildiz and Amira were discussing their favorite foods. In line 1, Meryem reacts to someone else's mention of KFC (Kentucky Fried Chicken), to which Amira appears to mention her 
favorite meal at this fast-food chain in line 2. Mr. de Jong then starts a question, which is indicated by the modal particle toch in line 3, which is similar to the English tag question 'right?' but can also occur in midsentence position (Englert 2010). Even before he says the word 'halal,' however, Meryem already reacts. This can be interpreted as 'recognitional onset' (Jefferson 1984, 12): Meryem anticipates on what Mr. de Jong is going to say and already starts her response. Indeed, in line 5, Mr. de Jong finishes his question with the word 'halal' (meaning allowed according to Islam). The question does not display elements of a question-as-challenge (cf. Koshik 2003): rather, it sounded like he was genuinely interested. This shows his orientation to these pupils as people for whom it is relevant whether food is halal, thereby making relevant the membership category of Muslim, even if it remains implicit. Meryem's reaction in line 4 endorses Mr. de Jong's orientation to them as people who might care about food being halal, but offers an account of why 'it does not matter': 'it is delicious.'

Amira laughs in line 6, and then says, with smile voice, that Meryem and Yildiz "are Hollanders" (line 8). This turn sounds as a tease directed at Meryem and Yildiz. Her categorization of Meryem and Yildiz as Hollanders does the work of suggesting that Hollanders do not care about halal food, and because Meryem and Yildiz say they do not care, they can be categorized as members of that category. The tease targets Meryem and Yildiz in two ways: it constructs the teacher's question as having 'called them out' for breaking rules that they oriented to as important in other moments, and it constructs the girls as members of an unprestigious category. Yildiz's reaction to Amira's mockery suggests that she has recognized the turn as a tease (hence the smile voice at the end of her turn) but that she also wants to set the record straight (cf. Drew 1987). By saying that Amira engages in the same supposedly transgressive behavior, Yildiz implies that Amira is not in the position to judge.

Although the interaction in Example 3.4 illustrates that pupils commonly oriented to Hollanders or Nederlanders as not being Muslim, the next two examples show how pupils more explicitly debated and challenged presupposed relations between ethno-national categories and religiosity. In Example 3.5, Omer, who frequently categorized himself as 'Turk,' was assumed to also be a member of the category 'Muslim.' He actively contested this, however.

Example 3.5. Field notes, 12 December 2017.

Care and Well-being class. Today, the pupils have to make lasagna but there is a problem. Normally, the school orders the meat used for the cooking classes from a halal butcher, but it seems that the food supplies order has gone wrong and there is only 
Negotiating the characteristics of Turken, Marokkanen, buitenlanders, and Nederlanders 81

'regular,' non-halal minced meat left in the freezer. The pupils are upset: they keep asking why they weren't simply told to bring minced meat from home. Ms. Smit is also annoyed with this mistake. Her solution for now is to tell the pupils that if they want, they are allowed to change the recipe and prepare their lasagnas without meat. She then assigns Sanne with the task of dividing the available meat among the pupils who do not need it to be halal. I see Sanne going around the kitchen counters, giving meat to the pupils categorized as Nederlanders while skipping the others. When Omer asks her for a portion of the minced meat, Sanne emphasizes that it's not halal. Omer responds that he "doesn't care" in a demonstrative tone and proceeds to prepare his lasagna with the meat.

During my fieldwork, Omer categorized himself as a Turk 'Turk' and often compared 'Turks' to 'Moroccans' and 'Dutch people' or explained the differences between categories to me. He thus subscribed to the same categorization scheme as the other pupils. This scene illustrates, however, that even if pupils seemed to uphold a strict, delineated categorization system, there were many differences in their understandings of what it meant to be a member of a category. While some pupils treated being Muslim or eating halal food as an inherent aspect of being a 'Turk' or 'Moroccan,' others, like Omer, challenged that relation. A great deal of complexity and variation thus hides behind the seemingly orderly scheme of categories depicted in Figure 3 (page 70).

It sometimes happened that people displayed characteristics that were usually constructed as indicative of other categories. This happens in Example 3.6, in which a pupil (Cindy) who was likely categorizable as Nederlander - a category that, as illustrated earlier, pupils did not associate with being Muslim - displayed knowledge of Islam in conversation with pupils who categorized themselves as Marokkaan or Turk. During my fieldwork, I did not hear Cindy categorize herself in ethno-national or religious terms, nor did I hear anyone else categorize her. Considering that most pupils who refrained from selfcategorization were, according to the local categorization logic, Nederlanders, there is reason to believe that Cindy was also categorizable as such. The interaction occurred during Care and Well-being class, and the pupils were sitting at a long table, eating the meals they had prepared as part of the class. I was sitting with them at the end of the table but did not participate in the interaction. The transcript starts when Leila - who categorized herself as Marokkaan - comments that she would like to get her ears pierced. Amira and Khadija also categorized themselves as Marokkaan, and Hatice and Yildiz categorized themselves as Turk. 


\section{Nederlanders and buitenlanders}

Example 3.6. 4 October 2017. Care and Well-being. Participants: Leila (L), Amira (A), Hatice (H), Yildiz (Y), Cindy (C), Khadija (K).

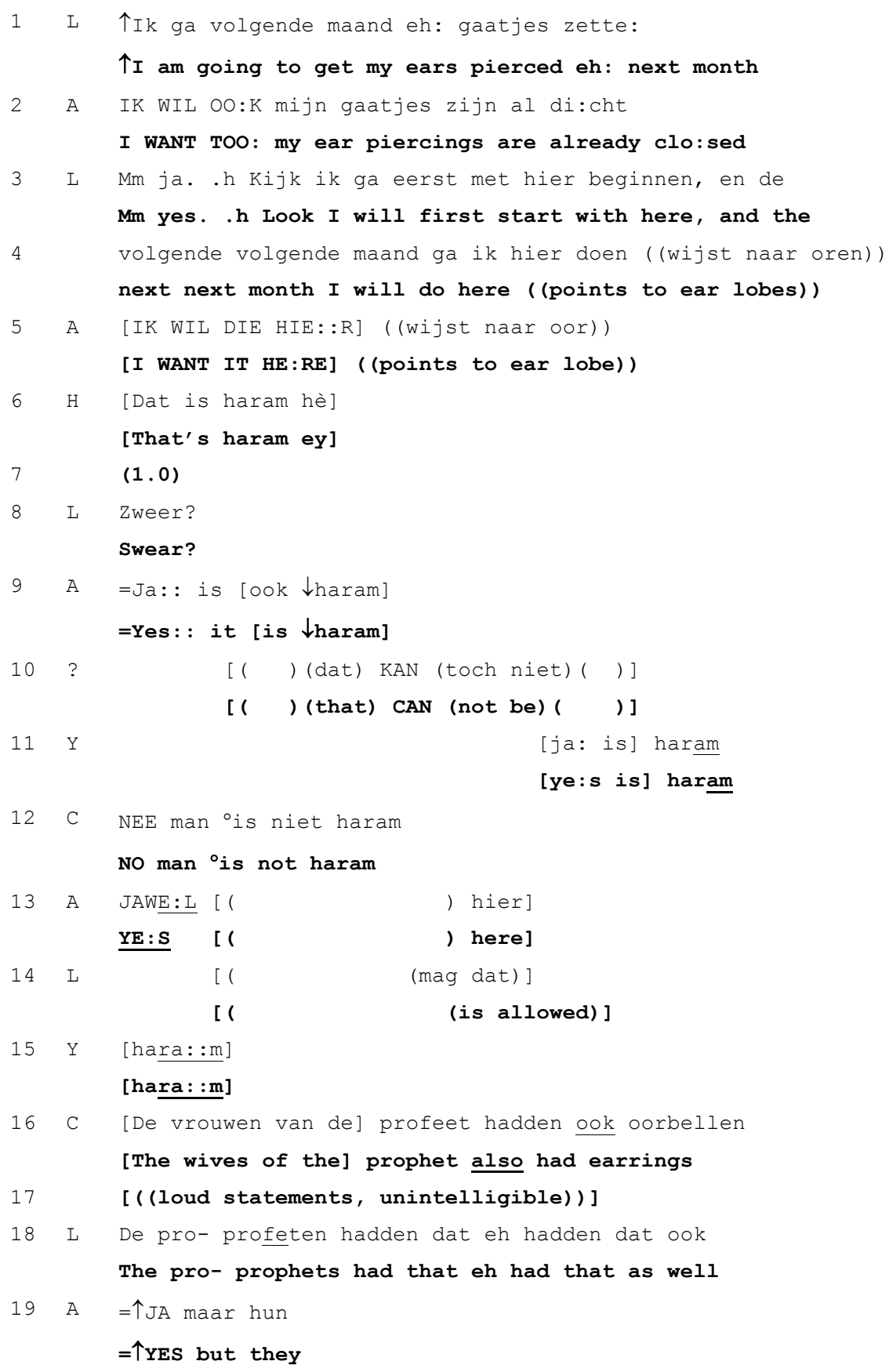


Negotiating the characteristics of Turken, Marokkanen, buitenlanders, and Nederlanders 83

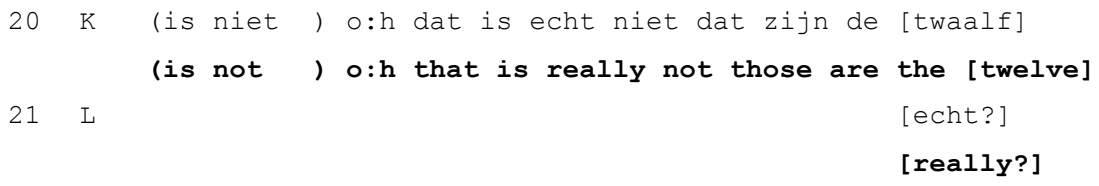

At the start of the interaction, Leila announces that she wants to get various piercings in her ear. Immediately in line 2, Amira aligns with her, saying that she also wants new ear piercings. They discuss where exactly they want those piercings, until in line 6, Hatice comments that it is haram.' As illustrated in Example 3.4 and Example 3.5, pupils who categorized themselves as Muslim often spoke about whether certain things (foods, behavior, etc.) were 'haram' (i.e. not allowed in Islam) or 'halal' (i.e. allowed). Therefore, even though this interaction features no labels, the membership category 'Muslim' is made relevant when the conversation turns whether piercings are 'haram' from line 6.

The tag question 'hè?' (line 6) has been observed to function mostly to solicit agreement (Englert 2010), but here, it seems to give Hatice's claim the status of a reminder, that is, it suggests that it is knowledge that Leila may, but does not necessarily, share. It furthermore shows Hatice's orientation to Leila and Amira as people for whom this information is relevant. The pause of 0.8 seconds before Leila responds with "swear [that it's true]?" indicates trouble in this turn (Stokoe \& Edwards 2009), probably caused by surprise or disbelief. In line 9, Amira confirms Hatice's claim, showing that this is not new information to her, but also implying that this is less important to her, in this moment, than the wish to get piercings. At the same time, two other pupils are still debating whether Hatice's claim is true or not (lines 10-11).

Then Cindy, who was sitting at an adjacent table, produces her first turn in the interaction (line 12), denying Hatice's claim without a suggestion of hesitation. Amira and another pupil respond to Cindy at the same time in lines 13 and 14. Although they disagree with her, they respond to the content of what she has said and thereby legitimize her participation in the conversation. This is striking, since pupils usually did not associate the category Nederlander with epistemic rights to, or access to knowledge about, the rules of Islam (Raymond and Heritage 2006). In line 15, Cindy produces her second turn and states that the wives of the prophet also had piercings (and, implicitly, that therefore it cannot be 'haram'). She thus justifies her knowledge as coming from an unquestionable authority, namely the behavior of the Prophet and his wives. This more elaborated display of expertise does not receive immediate reaction from any of the other girls, until Leila, in line 
18, repeats what Cindy said. By reproducing Cindy's comment, Leila shows acceptance and ratification of Cindy's comment (cf. Tannen 1987).

The interaction in Example 3.6 shows a striking negotiation of epistemic status, that is, we see "members' methods for managing rights to identitybound knowledge in self-other relations" (Raymond and Heritage 2006, 678). Even though pupils categorized as Nederlander were not generally associated with knowledge (or concerns) about 'halal' or 'haram' (see Example 3.4), in this case Cindy claims, and is granted, epistemic rights. The example also shows how pupils grappled with different, sometimes contradicting, categoryrelated expectations. Whereas on the one hand, the categories Turk and Marokkaan implied an expectation of being preoccupied with what is 'halal' or 'haram,' on the other hand, pupils often associated these categories with stances that favored rebellion and a relaxed attitude toward rules (as will be explored in Section 3.3.5). This interaction appears to illustrate some tensions stemming from such contradicting category-incumbent features. Leila, for example, seems to orient more to the aspect of doing being a Marokkaan that shows investment in whether something is 'haram'; whereas on this particular occasion, Amira appears to orient more to the 'rebel' aspect of doing being a member of that same category.

\subsubsection{Categorization and relations to physical appearance}

Perceptions of category membership in class $3 / 4 \mathrm{~b}$ hinged on many intersecting factors that are not easily taken apart. Religion, physical appearance, behavior, and many other things affected whether, or to what extent, people were seen as a typical member of a category. For instance, as seen in 3.3.2, (some) pupils perceived membership of the categories Marokkaan and Turk to be related to religion. As the following scene illustrates, they also perceived category membership to be related to physical appearance.

One time during my fieldwork, Hatice (who labelled herself Turk) and Jennifer (who later in the same conversation was labelled zigeuner or 'Gypsy' by Hatice ${ }^{17}$ ), were talking about the Catholic tradition of communion.

\footnotetext{
${ }^{17}$ The category zigeuner or 'Gypsy' was not part of pupils' daily discourse as I witnessed it during my fieldwork; this conversation was the only time I heard this category being mentioned. I therefore am not sure what it meant to pupils, and whom they categorized as such. A teacher told me that two or three of the pupils in class $3 / 4 \mathrm{~b}$ were van het kamp ('from the 'camp"), which is a common way to refer to Roma, Sinti, and 'travellers' in the Netherlands (e.g. Dirks 2018). The term zigeuner is sometimes used to refer to the same group but is often experienced as a stigmatizing term.
} 
Negotiating the characteristics of Turken, Marokkanen, buitenlanders, and Nederlanders 85

Jennifer had asked Hatice whether 'they' - I assume she was referring to the category of 'Muslims' or 'Turks' - had something like communion. When Hatice asked what a communion was, Jennifer looked at me for help to explain. I told her that I did not know how to explain because I am not Catholic, to which Hatice stated: "But you also don't look like a Nederlander" (field notes, 23 October 2017). She thus interpreted my not being Catholic as an exception to what was still expected of the category Nederlanders. ${ }^{18}$ This, in turn, she seemed to regard as compatible with the way in which my physical appearance, to her, formed an exception to what Nederlanders typically look like. In that sense, categories were often "protected against induction" (Sacks 1995, I, II:336): if individual members lacked a characteristic that was considered to be central to their category, that member would be seen as an exception to what remained unrevised 'knowledge.'

Just as there were people who, like me, were said to 'not look like a Nederlander,' often because of their dark hair and/or skin color, the opposite also occurred. Amine, for example, categorized himself as a Marokkaan but his physical appearance was different from what he (and others, as apparent from the extract) construct as typical for that category. On two occasions, Amine asked me whether I would have guessed he was Marokkaan, had I not known him. The first occasion happened right before the interaction transcribed in Example 3.7 below, the other about half a year later, in January 2018. The first time he asked me this, I was surprised by his question and told him that I was not sure. The conversation continued as transcribed below.

Example 3.7. 16 June 2017. Care and well-being class, Amine (A), Stefan (S, did not participate in this part of the interaction), and the researcher (Pomme, $P$ in the transcript).

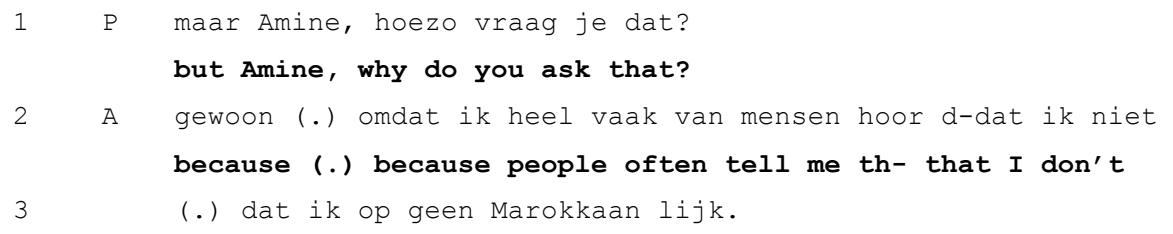

${ }^{18}$ Hatice and Jennifer's orientation to Catholicism as the 'standard' or expected religion of the people that pupils categorized as Nederlanders shows an orientation to the religious denomination that is historically and culturally dominant in Limburg rather than the Netherlands as a whole (CBS, 2016). The province of Limburg is predominantly Catholic, in contrast to more Central and Northern regions of the Netherlands, which are traditionally Protestant. 


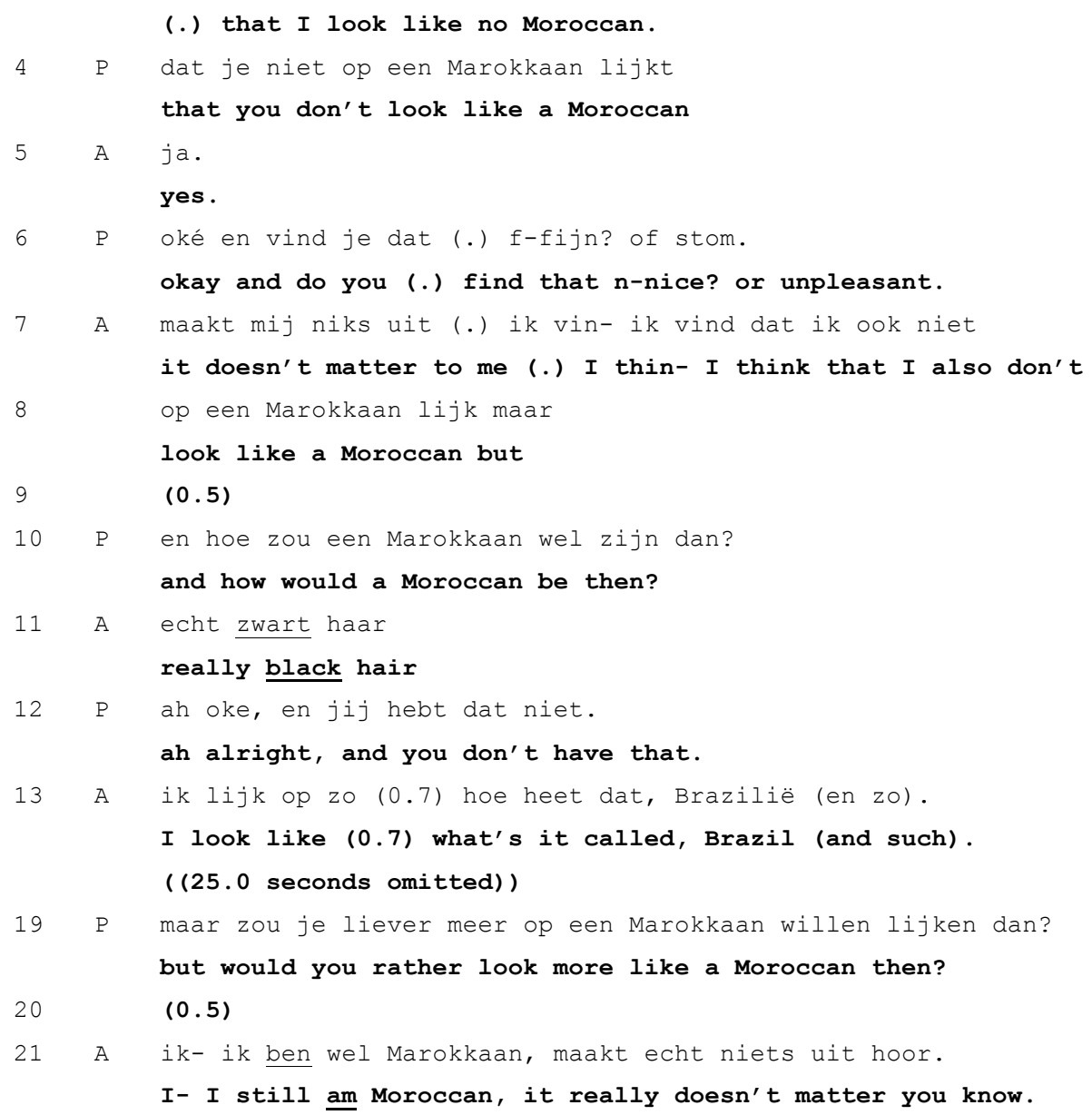

In this extract, Amine refers to a discourse on the physical appearance associated with the category Marokkaan, to which he feels he forms an exception (as others do too, apparently). I try to elicit his opinion on this in line 6. Amine provides a dispreferred answer: he does not elect any of the two (rather limiting) options that I provide to him (fijn 'nice' or stom 'unpleasant/bad'), but states that 'it doesn't matter' to him (line 7). It appears like Amine's question was aimed at finding out how essential physical appearance is to others to being perceived as a member of the category Marokkaan, but as I have turned it around by trying to find out how important it is to him, he states it does not matter. In the rest of the conversation Amine keeps giving short answers without elaborating much (lines 11 and 13), and there are some noticeable silences (lines 9 and 20). This suggests that Amine is uncomfortable with my persistent questions. When, in line 19, I again insist 
Negotiating the characteristics of Turken, Marokkanen, buitenlanders, and Nederlanders 87

on eliciting some kind of emotive evaluation, Amine again provides a dispreferred response: he does not say 'yes' or 'no' to my closed question whether he would 'rather look more like a Moroccan.' Instead, he emphasizes that he is Marokkaan anyway, so that it really does not matter (line 21). He thereby authenticates his own category membership by debating the assumption that the essence of category membership is physical appearance, i.e. that one has to look like a Marokkaan in order to be a Marokkaan. This statement, as a kind of assessment, could be interpreted as a way to end this conversation in which I did not at all answer his initial question. The conversation continued as transcribed in Example 3.8, when Amine's classmate Stefan adds a comment to the interaction, giving a new impetus to the topic.

Example 3.8. Continuation of conversation transcribed in Example 3.7. Stefan (S) has now joined the conversation.

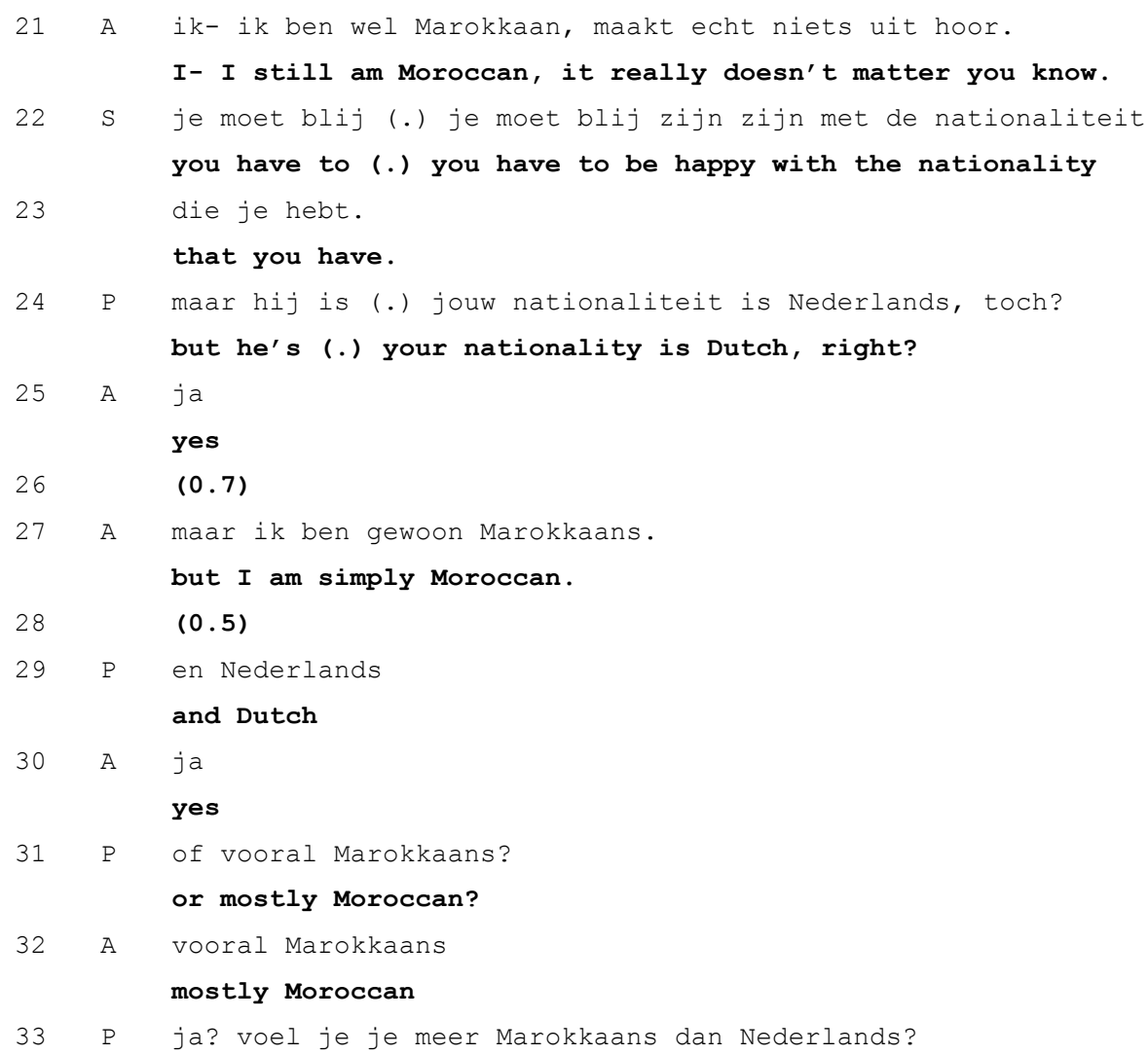




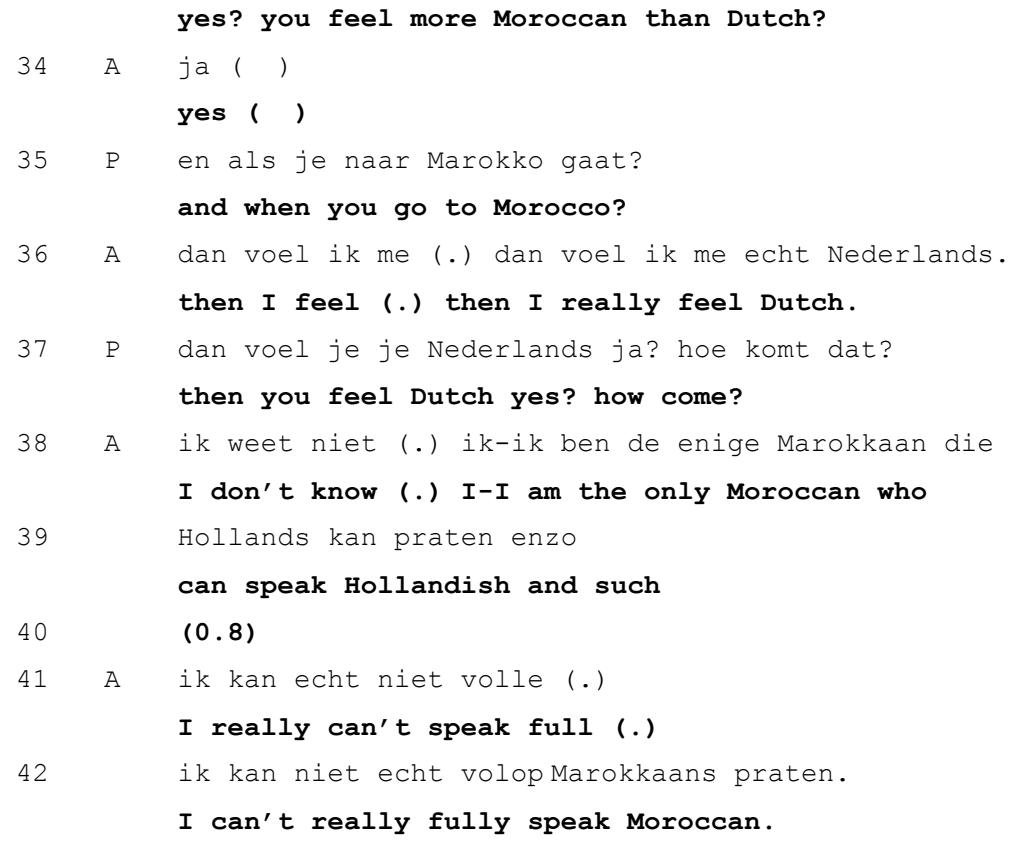

By stating that 'you have to be happy with the nationality that you have' (lines 22-23), Stefan sparks a further departure from the topic of physical appearance introduced by Amine at the start of Example 3.7. I had not heard the pupils talk about nationality as a determinant of category membership before and in my confusion seek to confirm with Amine that his nationality is Dutch in line 24. First, Amine gives a short preferred answer (line 25), but after a pause adds that he is 'simply Moroccan' (line 27). This invalidates - at least for Amine in this moment - nationality as determinant of the membership category 'Moroccan,' or indeed, the category 'Dutch.'

In the final lines of the extract (38-42), Amine engages with my question (of line 37) about his 'feelings' of being 'Moroccan' or 'Dutch' in Morocco. He speaks about language proficiency to contextualize his feelings of being Nederlands when in Morocco. Notably, he does not use the category label Nederlander (noun) but the adjective Nederlands when he refers to himself: $\mathrm{He}$ is not a member of the category Nederlander, but the adjective Nederlands is a description that can apply to him. Amine's self-categorization in line 38 remains defined by the category Marokkaan but is now complemented with a quality (speaking Hollands 'Hollandish' ${ }^{19}$ ). When he is outside the regular

19 Rather uncommonly, Amine refers to the Dutch language as Hollands ('Hollandish') instead of Nederlands ('Dutch'). This had not happened before and did 
context within which this categorization occurs (i.e. the Netherlands), there is a shift in orientation from the opposition Marokkaan-Nederlander, in which he identifies with the former, to categorically different kinds of Marokkaan. There is one kind in the Netherlands (like himself) and another in Morocco. The other kind of Marokkaan can be understood to live in Morocco and speak 'Moroccan,' unlike Amine (line 41-42). Amine's lack of proficiency in 'Moroccan' (it is unclear whether he refers to Moroccan Arabic or Berber) makes him feel like an outsider in Morocco, but he still uses the label Marokkaan to refer to himself.

In Example 3.7 and 3.8, we have seen a negotiation of perceived essences of category membership: is it defined by one's physical appearance, nationality, 'feeling,' or by language proficiency? At first, Amine seems preoccupied with the issue of his perceived deviance from expectations regarding physical appearance, and whether that makes other people (i.e. me) recognize him as a Marokkaan. When I push for his own evaluation on this issue, he constructs it as unimportant. Regardless of perceptions, he 'simply is Moroccan.' When in the context of Morocco, he constructs language proficiency as more central to category membership: being there makes him 'feel' more Dutch because he speaks Dutch rather than 'Moroccan.'

\subsubsection{Language- and clothing styles as indicative of categories}

Pupils of class $3 / 4 \mathrm{~b}$ also sometimes spoke about associations between wearing certain clothing, accessories, or ways of speaking, and being a member of the category buitenlander. In the interaction transcribed in Example 3.9, I asked some pupils about language practices of 'people who are not Moroccan' (my words in line 3; Amira and Meryem use 'they' in the subsequent lines). This sparks a discussion of some styles that they associated with categories.

Example 3.9. 16 June 2017. Group conversation with Farida (F), Yildiz (Y), Meryem (M) (who categorized themselves as 'Turks'), Amira (A), Dounia (D) (who categorized themselves as 'Moroccans'), and the researcher (Pomme, $\mathrm{P}$ in the transcript).

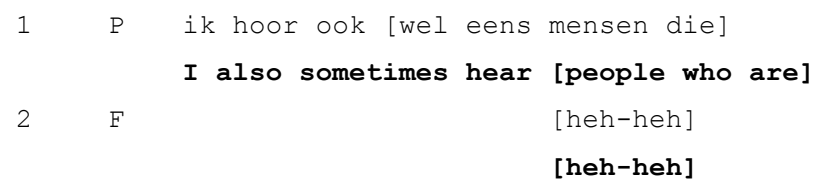

not happen after, and it is unclear to me why Amine might have done so in this context. 


\section{Nederlanders and buitenlanders}

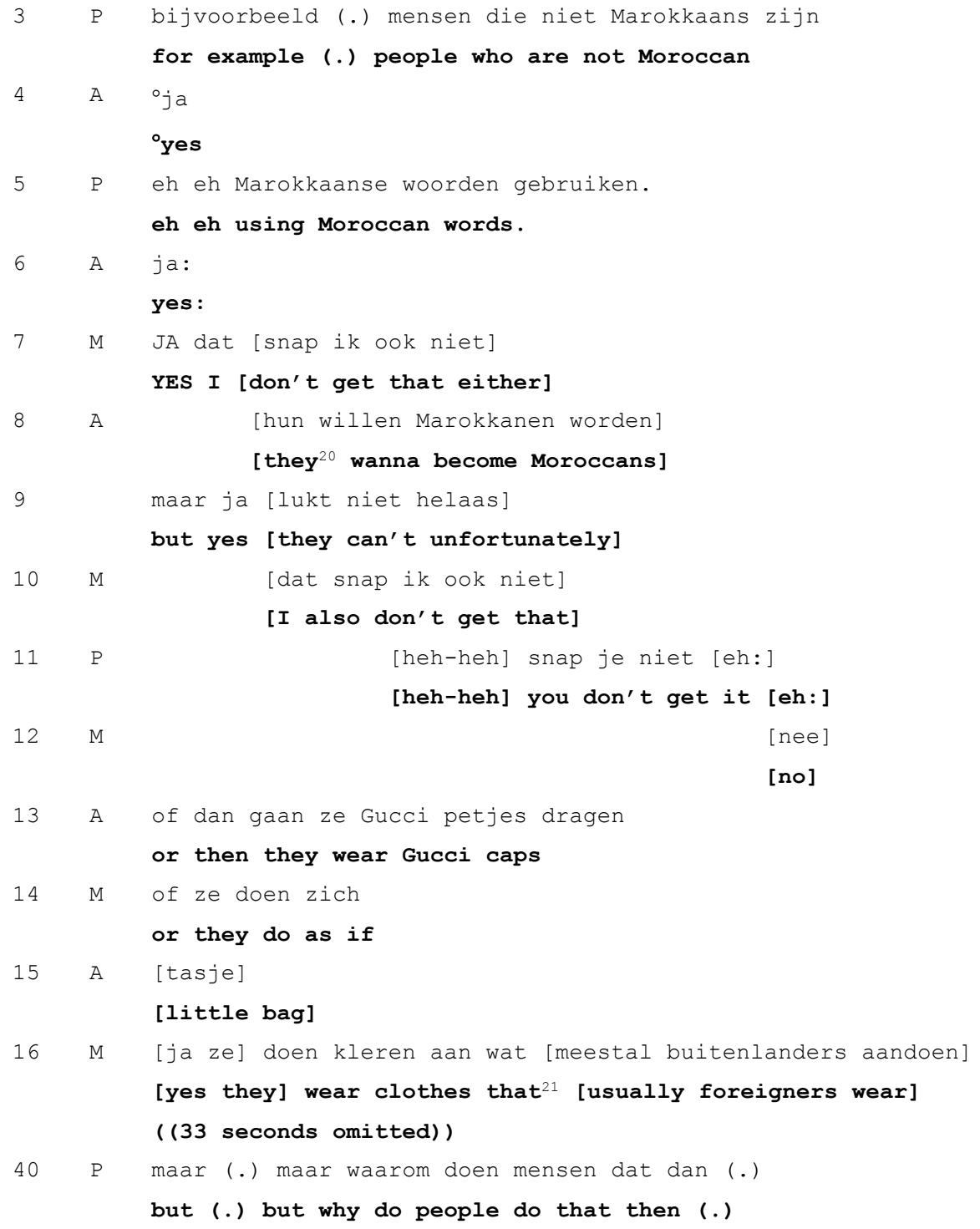

${ }^{20}$ In Dutch, Amira's use of hun in the subject position (as Meryem's in line 44) is regarded to be sub-standard Dutch. I translate it into a standard English phrase using 'they' because its use is widespread among people with different socioeconomic backgrounds, ages, ethnicities, regional varieties, etc., in the Netherlands.

${ }^{21}$ The use of 'wat' in this phrase is regarded to be non-standard Dutch. I have translated it into standard English for the reason described in note 20. 
Negotiating the characteristics of Turken, Marokkanen, buitenlanders, and Nederlanders 91

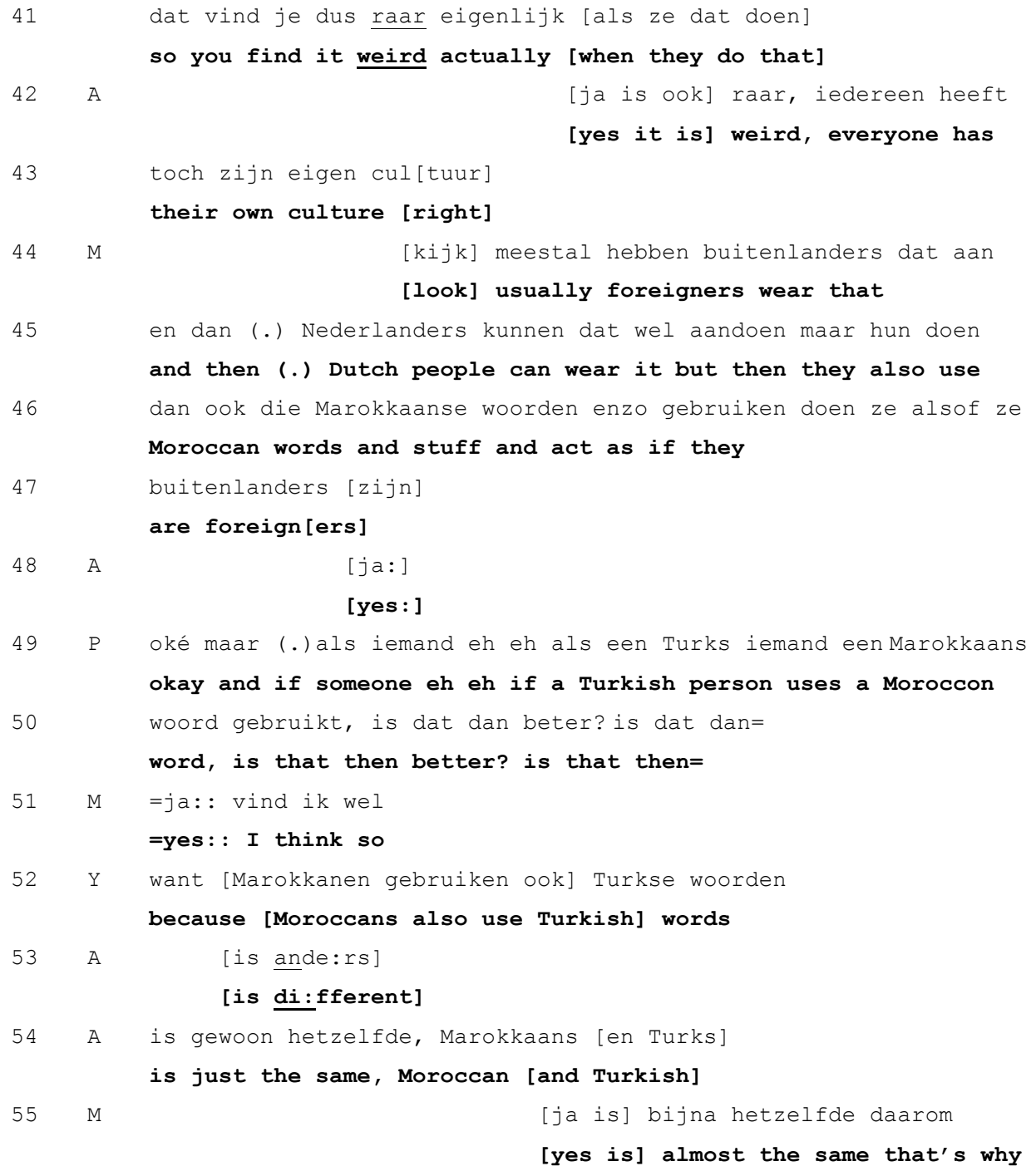

My statement in lines 1-3-5 about hearing people 'who are not Moroccan' using 'Moroccan words' launches Amira and Meryem into a conversation not about language, but about 'people who are not Moroccan' versus 'Moroccans' (line 8). When 'people who are not Moroccan' want to act like 'Moroccans,' they take on what Amira and Meryem have constructed as 'their' style. According to Amira, who categorized herself as 'Moroccan,' people use 'Moroccan' words to 'try to become Moroccans.' They thus construct language as a factor that defines category membership. The kind of language they speak 
about is not the same as the one Amine spoke about in Example 3.8, however. Amine mentioned not being able to 'fully speak Moroccan,' which led him to feel 'Dutch' in Morocco. These pupils in Example 3.9 refer to the practice of inserting 'Moroccan words' into utterances that are otherwise regarded as Dutch as indicative of the membership category buitenlander 'foreigner.'

Meryem comments that 'she does not get it either' (line 7), implying that I asked about it because I also do not 'get it.' During my fieldwork, however, I observed that Meryem categorized herself as a 'Turk' and often used Moroccan words. Strictly speaking, she thus engaged in the practice about which I asked. Meryem does not seem to feel personally implicated, however, but constructs the behavior as something that 'others' do. In fact, in line 16, she changes the category we are discussing from 'Moroccans' to 'foreigners' and thereby includes herself in the imitated category. This is not marked nor taken up as a change in topic: she presents the style indexed by speech and accessories as categorically congruent when performed by any kind of 'buitenlander' (thus including herself). The language practice of 'using Moroccan words' is thus constructed as a central characteristic not only of the category Marokkaan, but of the category buitenlander more generally. This equation of Marokkanen and buitenlanders in terms of language and dress style continues in the rest of the conversation. In line 42, Amira stresses that it is 'weird' when people use language and display signs that she perceives as incongruent with their category, because 'everyone has their own culture.' She constructs the category Marokkaan as a 'culture' that one 'has,' which is shared (or shareable) between Turken and Marokkanen and desirable but unreachable to others.

The pupils thus assigned 'ethnic' meanings to language, clothing, and accessories, but this 'ethnic' reading is rather generalized: the main opposition built in the interaction is one between buitenlanders (including and equating Turken and Marokkanen) and Nederlanders. This might have to do with recipient design (Sacks, Schegloff, and Jefferson 1974): during the fieldwork, they had categorized me as a Nederlander and may have assumed that I was oblivious to differences between different 'kinds' of buitenlanders. Another possibility is that, for these pupils, the categories Turk and Marokkaan were sufficiently similar with regard to certain locally indexed category incumbent characteristics and activities (for example dressing or speaking in a certain way), that they could use the labels interchangeably in discussions of those particular characteristics. Either way, their equation of Turken and Marokkanen shows that the pupils shaped these categories in a locally specific way and as embedded in the Netherlands, where they could be characterized, among other things, by language and clothing. 
Negotiating the characteristics of Turken, Marokkanen, buitenlanders, and Nederlanders 93

A few minutes later in the same conversation, I asked the girls why they thought people display such 'incongruent' behavior. This brings up the topic of status differences between the different categories in class 3/4b and South High School more explicitly.

\section{Example 3.10. Continuation of Example 3.9.}

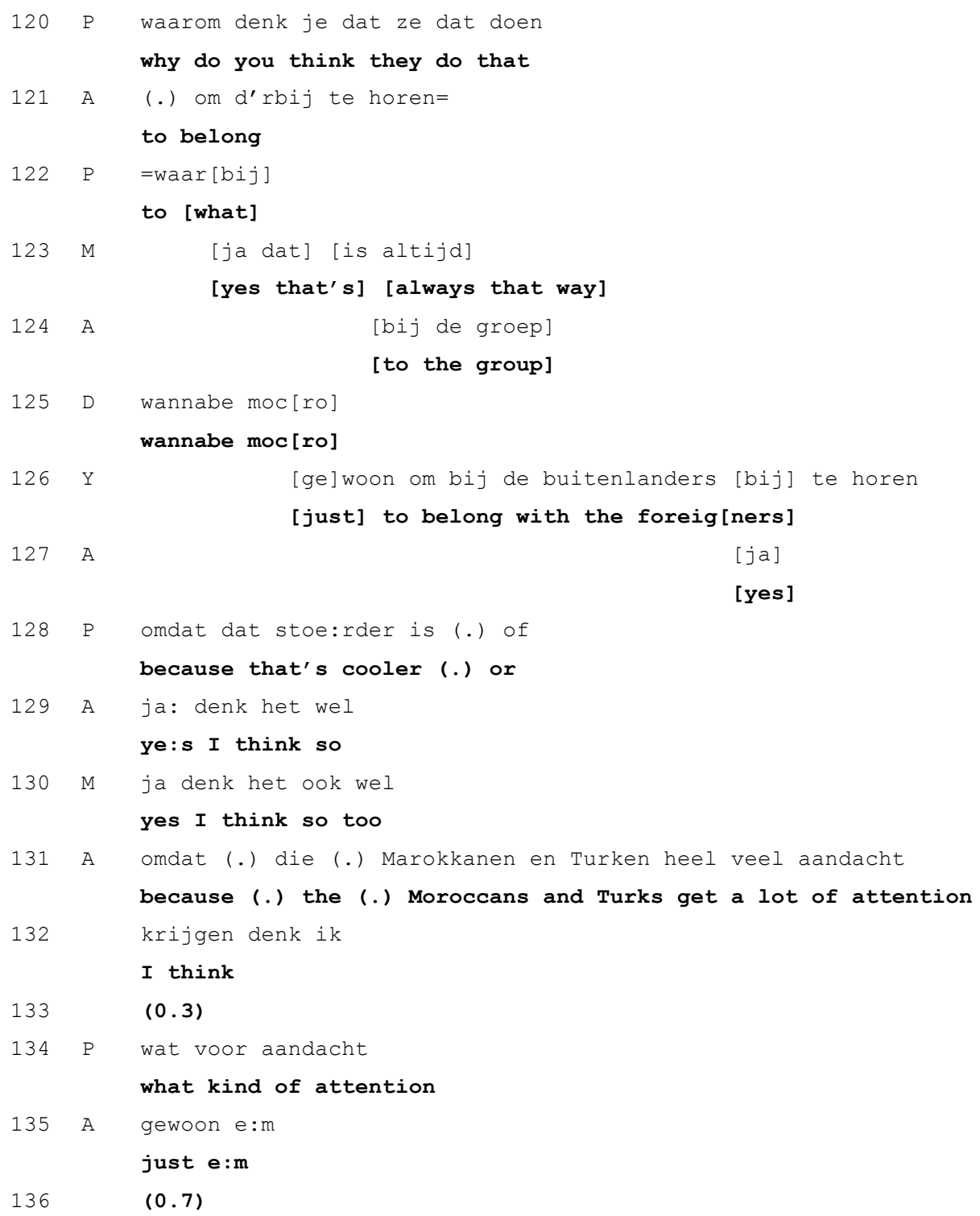

$136 \quad(0.7)$ 


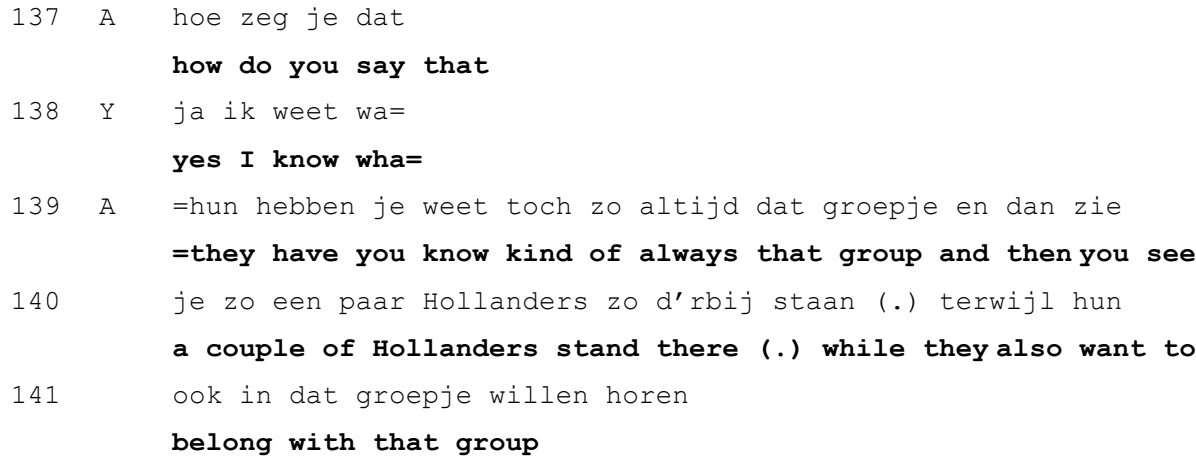

In this extract, the categories buitenlander and Marokkaan are again used interchangeably. Yildiz speaks about 'foreigners' (line 126) and Dounia uses the term 'wannabe mocro' in line 125 ('mocro' functions as a synonym for Marokkaan in this context ${ }^{22}$ ). Together, Amira, Yildiz and Meryem construct the display of characteristics that they associate with being a Marokkaan or buitenlander, when performed by a Nederlander, as an effort to appear stoerder 'cooler' - which is the adjective that I offer in line 128. In the rest of the interaction, they further elaborate on the image of the Nederlander (or Hollander, line 140) who uses certain clothing and associates with others labelled Marokkaan, as a 'wannabe,' thereby positioning the categories buitenlander, Turk and Marokkaan as eligible for imitation in order to raise one's status.

In class $3 / 4 \mathrm{~b}$, readings of ethnicity thus invoked local social hierarchies and negotiations of (in)authenticity. I further explore this in the next section, which describes some character traits and behaviors that pupils often associated with categories.

\subsubsection{Categorization and debates about behavior}

In South High School, the categories buitenlander, Marokkaan and Turk carried prestige. Pupils made associations between those categories and being 'cool,' tough (see Example 3.10), challenging authority, but also, for example, having a good sense of humor (see Example 4.3, later in Chapter 4). This local prestige meant that their associated characteristics were liable to what was

${ }^{22}$ The word 'mocro' for Marokkaan 'Moroccan' used to be associated primarily with youth varieties, and was popularized around 2004 through popular music. Today, however, it has been said to have 'lost its innocence' due to its increased use in media in the context of the so-called 'mocro maffia,' a branch of organized crime known for its violence, led primarily by criminals of Moroccan, but also Dutch and Antillian, descent (Derbali 2018). 
Negotiating the characteristics of Turken, Marokkanen, buitenlanders, and Nederlanders 95

regarded to be imitation (cf. Schegloff 2007), as illustrated previously in Examples 3.9 and 3.10. Pupils mostly linked the category Nederlander, by contrast, to undesirable traits, as can be seen in the examples in this section (Example 3.11 and 3.12). Example 3.11 occurred during an informal focus group conversation during a class in which the teacher allowed me to talk to a few pupils separately in an empty classroom.

Example 3.11. 16 June 2017. Participants: Amira (A), Dounia (D), Meryem $(\mathrm{M})$, Yildiz (Y), and the researcher (Pomme, $\mathrm{P}$ in the transcript).

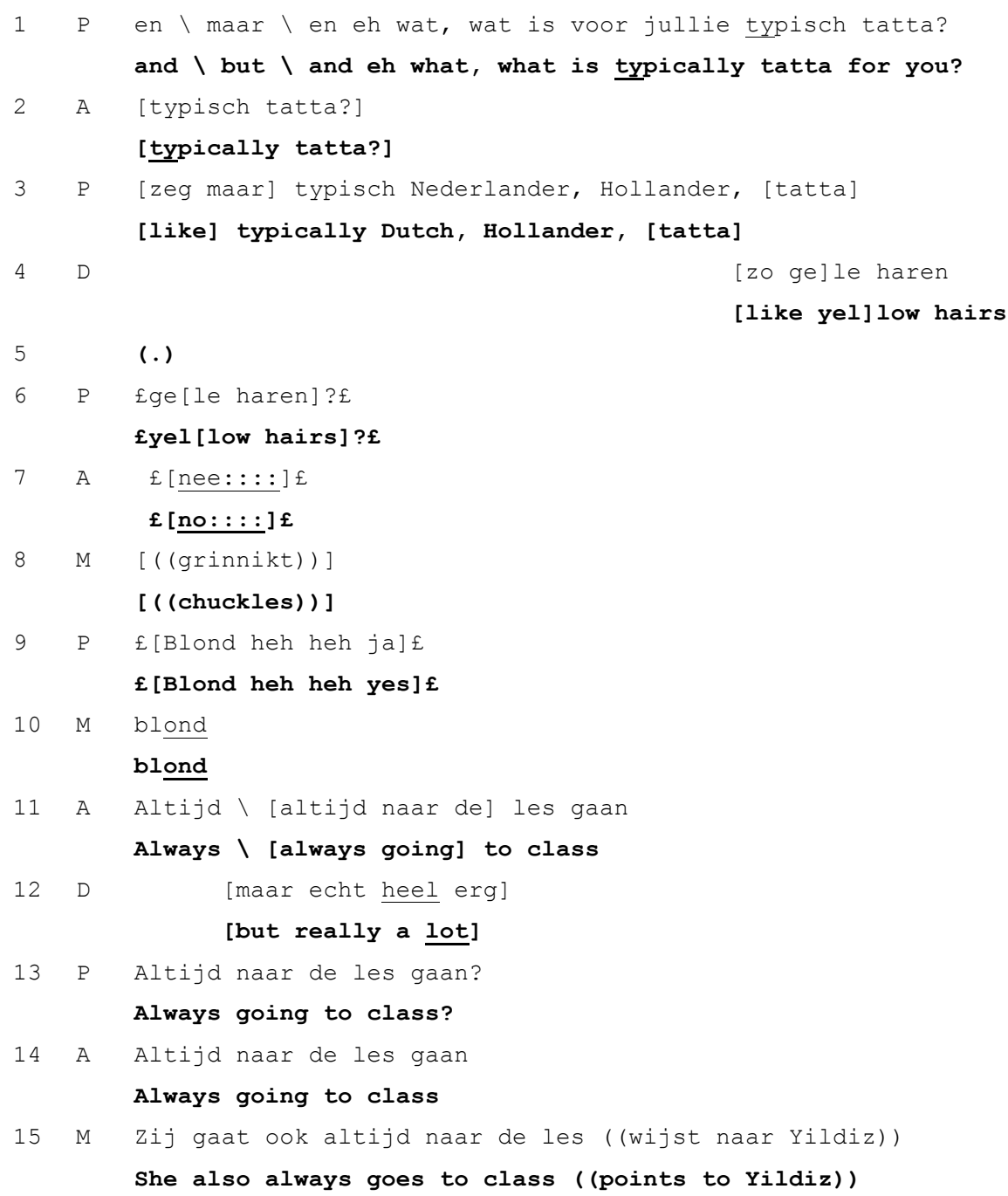




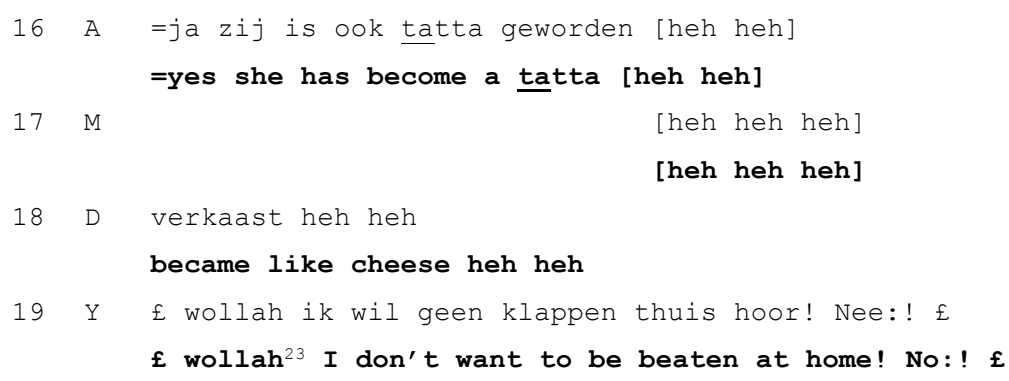

In this example, I ask some pupils what they think is 'typically tatta.' When I do not get an immediate response, I elaborate by using the other terms that were common among them for the same category (Nederlander, Hollander). Dounia responds with a physical characteristic ("yellow hairs," line 4), which is rejected by Amira (line 7), and makes Meryem laugh (line 8). Meryem and I 'correct' the word 'yellow' by replacing it with 'blond.' Amira also disagrees, but this seems to address Dounia's framing of this physical characteristic as essential to the category tatta, as she gives an alternative answer: always going to class. This is in line with how pupils often characterized members of the category Nederlander, namely as people who always follow the rules. Meryem immediately challenges this theory, however, by saying that Yildiz - who categorized herself as Turk - also always goes to class. In lines 16-18, Amira and Dounia solve this incongruence by laughingly pointing out that Yildiz 'has become a tatta.' This tease is very similar to that in Example 3.4 (page 79), and Yildiz reacts to it in a similar way, namely by setting the record straight (Drew 1987), this time by means of an account ('I don't want to be beaten at home,' i.e. it is because of her parents' wish, rather than her own), while her smile voice also displays recognition of the jocular intent.

Another common discourse among pupils was that tatta's are stingy. In Example 3.12, Omer elaborates on this. Just before the transcribed interaction, Omer said he disliked Hollanders, to which I pointed out that Ben, his friend who was sitting next to him at that moment, was also a Hollander. "He is the exception," said Omer. I then asked why he disliked Hollanders ('those people' in line 1).

${ }^{23}$ 'Wollah' means 'I swear (to God).' This expression was used often among the pupils of class $3 / 4 \mathrm{~b}$. Its use is also widespread especially among youth in other parts of the Netherlands. 
Negotiating the characteristics of Turken, Marokkanen, buitenlanders, and Nederlanders 97

Example 3.12. 26 October 2017. Care and Well-being. Participants: Omer $(\mathrm{O})$, Ben (B), the researcher (Pomme, $\mathrm{P}$ in the transcript).

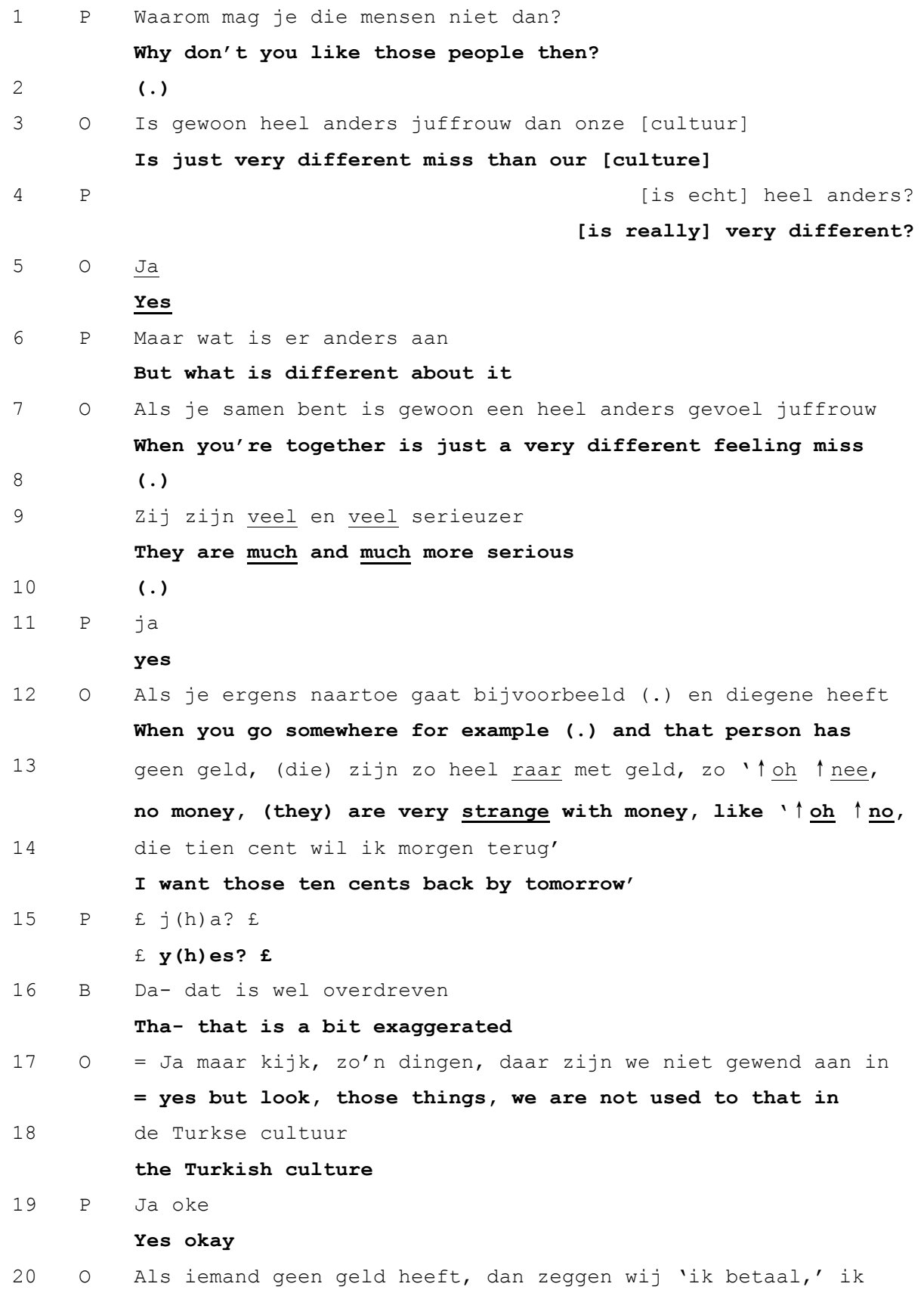




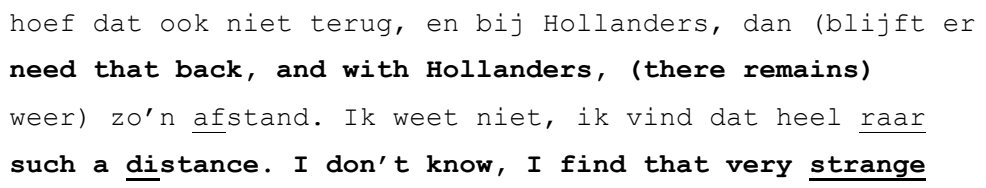

Here, Omer explicitly contrasts Dutch stinginess with Turkish generosity, thereby constructing and reproducing those as category-incumbent characteristics. When Ben, in line 16, remarks that that is 'a bit exaggerated,' Omer does not engage much with this comment, but instead further elaborates on the image of Dutch stinginess in contrast with 'the Turkish culture.' This discourse was recurrent in class $3 / 4 \mathrm{~b}$. Ahmed, for instance, also drew on the image of Dutch stinginess during a class discussion about an upcoming school excursion (which he did not want to attend, but others did), for example: "That's a real tatta right there: 'My parents have paid so I will go." Amira, Meryem and Dounia, on another occasion, added another category-incumbent feature when I asked whether they had friends who were Nederlanders. They replied: 'No, they are so boring. They don't have the same sense of humor' (for an analysis of that conversation, see Example 4.3 in Chapter 4).

Although those negative images existed, I saw plenty of examples of people who categorized themselves as buitenlander who were friends with others who were categorized as Nederlander. Even Omer, who seemed to enjoy mentioning how much he disliked Nederlanders, always sat next to Ben in class, who was categorized as a Nederlander. While pupils thus categorized one another and attached certain (positive or negative) features to those categories, they also 'particularized' and made room for exceptions.

\subsection{Conclusion}

In times of identity politics and heated debates about what defines national identity in a globalizing world, labelling practices of people born in the Netherlands, who refer to themselves as Marokkaan 'Moroccan' or Turk 'Turkish' and not Nederlander 'Dutch,' can be experienced as a threat to national integrity (Dagevos and Huijnk 2014). Such use of labels is routinely connected to, for example, experiencing a sense of unbelonging to the Netherlands, which is portrayed as a problem when people are expected (even required) to demonstrate feelings of emotional attachment to the country in which they live (Duyvendak 2011). This chapter has aimed to show that the categories Marokkaan, Turk, Nederlander and buitenlander have a complex 
meaning potential, acquire their meaning in their specific contexts of use, and even in such a local context are the topic of constant negotiation.

I suggest that even though the labels Marokkaan, Turk, and buitenlander are sometimes understood to indicate a kind of transnational belonging (e.g. Azghari, Hooghiemstra, and van de Vijver 2015; Verkuyten and Yildiz 2007), or seem to imply an essentialist understanding of themselves as 'non-Dutch,' to the pupils they stood for kinds of people within the Netherlands and within their local social context. The idea of descent functioned in conjunction with other features such as religion, physical appearance, style, or behavior, to construct membership categories with specific incumbent features and activities. All those features were hotly debated, however, in terms of how essential or central they were to category membership. There were recurring exceptions to the 'knowledge' associated with categories, and the prevalent categorization scheme did not prevent the occurrence of friendships across locally drawn categories. Daily social and linguistic practices were also less strictly divided than those categories seemed to suggest.

At school, the categories buitenlander, Marokkaan and Turk carried prestige. The positive connotations of buitenlander, Marokkaan and Turk present a striking contrast with the image of those same social categories in Dutch society generally, in which people with migration backgrounds are often presented as problematic. Especially the image of the 'Moroccan' in much national media is that of a 'folk devil' (Bouabid 2016): it is often associated with nuisance, criminality, or women's oppression. It is not coincidental that these pupils, specifically, did this work with ethnic labels. Having a migration background and, furthermore, being enrolled in the undervalued vocational tracks, they grappled with membership in two categories that are stigmatized in the Netherlands. Even if it is difficult to surmise their exact awareness of this, they were constantly reminded of the low expectations that were placed upon them. The fact that pupils with migration backgrounds formed a majority in many of the vocational track classes in South High School enabled them to change the indexicalities of those categories from a wider societal 'negative' to a local 'positive' in a sort of re-appropriation of stigmatizing labels, thereby (temporarily) alleviating the stigmatizing load of at least one of the categories by which the outside world defined them. At school, they could enjoy the power and prestige that they were not often accorded outside it. Pupils' labelling practices thus had locally occasioned meanings, but also ultimately reflect wider-spread practices of categorization and marginalization of people with migration backgrounds in the Netherlands. 
The next chapter (Chapter 4) further explores the associations and negotiations surrounding category constructions described in this chapter. Whereas the current chapter focused on the meanings or content of categories, that is, on the pupils' debates and negotiations surrounding the characteristics of categories, Chapter 4 will present a view on the functions of category mentions in interaction, that is, on how pupils mobilized those categories to achieve moves in interaction (e.g. joking, negotiating positions of power, (dis)aligning, etc.). 


\section{Interactional functions of ethnic labels}

Field notes, 28 June 2017

"STUPID MOCRO!" I look up from the kitchen counter of the Care and Well-being classroom to see what's going on. It's the end of the schoolyear and that means that the whole week, the pupils (and I) are cleaning this huge space: the four kitchen counters, all the pots and pans, the laundry closet, the hairdressing area, and all the other sections. "STUPID TURK!" I now see that Yildiz and Amira are the ones shouting, but they are also laughing, and I shift my attention back to the grease stains on the counter. I think back to when, at the start of my fieldwork, these kinds of exchanges surprised me. Meanwhile, the girls continue. "GO BACK TO YOUR OWN COUNTRY!" - "Go back to Israel!" Now my interest is sparked. Did I hear that right: Israel? "Go back to Marrakech!", Amira, who refers to herself as 'Marokkaan' tells Yildiz, who categorizes herself as 'Turk.' "Go back to Istanbul!', Yildiz replies. The girls laugh and continue mopping.

\subsection{Introduction}

A few days after the joking exchange between Amira and Yildiz described in the fieldnote above, I found out its background: in 2014, a young man who was part of a "pro-Wilders ${ }^{24}$ manifestation, proclaimed in a TV interview that "all Moroccans should go back to Istanbul, where they came from." The video was widely shared on social media. This man's statement was the perfect inspiration for a banter session among the pupils of class 3/4b: it was xenophobic, it targeted people like themselves, it was comical because of its ignorance, and it included a well-known disparaging phrase ('go back to where you came from'). At the start of my fieldwork, it surprised and sometimes shocked me when pupils would engage in such banter, especially when it sounded xenophobic or racist to me. But getting used to the goings-on in class $3 / 4 \mathrm{~b}$ meant getting used to those exchanges. They happened regularly, and

\footnotetext{
" Parts of this chapter have previously appeared in: van de Weerd, Pomme. 2019. "Those foreigners ruin everything here': Interactional functions of ethnic labelling among pupils in the Netherlands." Journal of Sociolinguistics 23 (3): 244-262.

${ }^{24}$ Geert Wilders is the leader of the (rather controversial) party Partij voor de Vrijheid, mostly known for their anti-immigration stances.
} 
they appeared to have an important social function: the pupils bonded through laughter. At the same time, the particular kind of humor about xenophobic remarks - of which we will see more examples throughout this chapter - can have more serious functions. The pupils who made fun of xenophobia or racism were the pupils who had migration backgrounds themselves and were thus targeted by such discourses. They were very aware of the stigma that came with being (seen as) a buitenlander, Turk, or Marokkaan. Humor and laughter, shared with friends who were in the same position, seemed to be a way for them to deal with discourses that targeted them, and might function to mitigate their hurtful effects. Ethnic label references in banter exchanges were thus not only funny, but they were central in enabling pupils to engage in particular communicative practices with important social functions.

As seen in the previous chapter (Chapter 3), pupils of class $3 / 4 \mathrm{~b}$ frequently referred to the categories Marokkaan, Turk, buitenlander, and Nederlander. In that chapter, I argued that these categories had local social meanings, and I set out to explore those meanings and the constant negotiations surrounding them. But the question of why these categories were so commonplace in the discourse of the pupils remains. One reason that may spring to mind is their age: adolescence (as a sociocultural construct, see e.g. Lesko 2012) is a period often characterized as one in which peer group affiliation becomes of prime importance. The construction of locally specific social categories during adolescence has been documented extensively in different cultural and historical contexts (e.g. "jocks" and "burnouts" described by Eckert 1989; "hotrodders" described by Sacks 1995; or "lads" and "ear'oles" in Willis 1981). The ways in which pupils who referred to themselves as Marokkaan or Turk talked about Nederlanders as behaving and looking a certain way often sounded remarkably similar to how the 'lads' described the 'ear'oles' in Willis' (1981) study, and how 'burnouts' described the 'jocks' in the study by Eckert (1989). In other words: processes of adequation and distinction, and negotiations of hierarchy, seem to occur in many adolescent peer groups, but they occur in different ways depending on the historical, geographical, cultural and social context (Brown, Eicher, and Petrie 1986; Eckert 1989).

In this chapter I will explore another potential reason, namely that references to the categories Marokkaan, Turk, buitenlander, and Nederlander were "[useful] in getting things done" (Whitehead 2012, 1261): they could be mobilized to achieve interactional moves such as (dis)aligning, joking, or negotiating power relations. The goal of this chapter is to contribute to a deeper understanding of ethnic labelling practices by highlighting such functions in interaction. 
The chapter is organized as follows. In the section hereafter (4.2), I will expand on the theoretical framework that informs my analyses. This includes a short overview of previous work on categorization and talk-in-interaction. The sections thereafter $(4.3,4.4$, and 4.5$)$ contain the analyses: I demonstrate how interactants altered power positions in the interaction through references to labels (Section 4.3); I discuss how pupils engaged in jocular mockery with ethnic labels (Section 4.4); and examine the social, categorical and interactional conventions and restrictions surrounding this kind of humor (Section 4.5). I conclude by arguing that pupils' labelling practices had locally occasioned meanings and functions, but that they ultimately reflect widerspread systems of categorization and marginalization of people with migration backgrounds in the Netherlands.

\subsection{Categories and action in interaction}

Much anthropological and linguistic scholarship examines the kinds of 'identities' that are constructed and negotiated through category talk. I have discussed some of this literature in Chapter 3. The current chapter takes a different approach by investigating what categorization and labels achieve in interaction, that is, the interactional functions of category references. Such an approach helps understand why people might be compelled to use labels to categorize themselves or each other in particular interactions.

When mentioning membership categories to engage in interactional work, people draw on presumed shared knowledge about those categories and put that knowledge to work. Stokoe \& Edwards $(2009,104)$, for example, discuss how a woman involved in a neighborhood dispute categorized herself as a 'single mother' - which, in their data, was tied to 'being tired' and 'not having resources to cope' - enabling her to give force to her complaint. A categorization thus served the broader function of complaining. The data of Stokoe \& Edwards (2009) also include many examples of category-based denials, in which people denied allegations by arguing that they were not "the kind of person" to do what they were accused of. In those cases, categorizations helped to deny culpability.

Edwards (1998) analyzes how a husband and wife in a counseling session switched strategically between the categories 'girl,' 'woman,' 'another woman' and 'married women' to conjure up different associations, which helped them attend to "local, rhetorically potent business in their talk" (Edwards 1998, 30). This included drawing upon cultural discourses surrounding (in)fidelity. Baker $(1997,78)$ shows how teachers in a school staff meeting categorized students in terms of their good or bad behavior in order to "persuade, to question, and to justify." In the context of phone calls to the doctor, kinship 
terms such as "daughter" and marital unit terminology such as "husband" or "wife" were found to enable people to signal the "non-accountability' and overall logic of their calling on another person's behalf (Kitzinger 2005).

For a long time since Sacks' early work on racial membership categories, there has not been much scholarship that "expressly considers how ethnicity is utilized by participants as a resource in [...] attending to the myriad exigencies of specific interactional moments" (Hansen 2005, 65; see also Whitehead and Lerner 2009). More recent work has shown, though, that references to ethnic and racial categories can indeed be mobilized to do interactional work similar to that being done, as in Kitzinger's (2005) example, by kinship categories or marital unit terminology. In Norwegian classrooms, for example, ethnic labels were "topicalized in situations of building and demonstrating alliances, of conflict and competition, and to escalate opposition when disputes had already surfaced" (Aukrust and Rydland 2009, 1538). Jurva and Jasinskaja-Lahti (2015) demonstrate how participants evoked ideas about being Finnish to account for a planned return migration to Finland. Whitehead (2012) shows how a speaker's self-categorization as 'white,' while praising Archbishop Desmond Tutu, "[draws] on commonsense knowledge about the categories of people (i.e. black South Africans) who would ordinarily, and historically, be understood as having been the primary beneficiaries of Tutu's work" (Whitehead 2012, 1252). Categorizing himself as 'white,' the speaker could be seen to add weight to his praise.

In all the examples just mentioned, the actions achieved by referring to categories are achieved by virtue of their shared web of associations. In Chapter 3, I have explored the range of associations that the categories Turk, Marokkaan, buitenlander and Nederlander carried in the context of class 3/4b, so that in this chapter, it may be clearer how those associations enabled pupils to achieve the interactional work that those category mentions achieved. The following sections present an analysis of the interactional work that ethnic labels were mobilized to do in class 3/4b. Analysis of a collection of 265 interactions in which pupils (and sometimes teachers) made references to ethnic labels resulted in the identification of common interactional contexts in which pupils of class $3 / 4 \mathrm{~b}$ labelled themselves or others. In the next sections, I describe some typical examples of interactional moves which were selected for their representativeness rather than for being unique in the context of this collection.

\subsection{Ethnic labels and power positions in interaction}

One of the actions pupils often achieved by referring to labels was to negotiate relative positions of power and/or expertise within interactions. This could 
mean taking on a position of expertise vis-à-vis someone who was categorized as member of a different ethnic category.

Example 4.1 is a clear instance of this. It occurred when I joined Omer and his friend Ben during a Care and Well-being class. Ben did not participate in the transcribed interaction. Before the start of the transcribed interaction, Omer said that he was tired because he came home very late the night before. I asked him what his parents thought of that, to which Omer replied that his father had nothing to say about him (i.e. did not have control over him). Then the following interaction took place.

Example 4.1. 26 October 2017, Care and Well-being. Participants: Omer $(\mathrm{O})$, researcher (Pomme, $\mathrm{P}$ in the transcript).

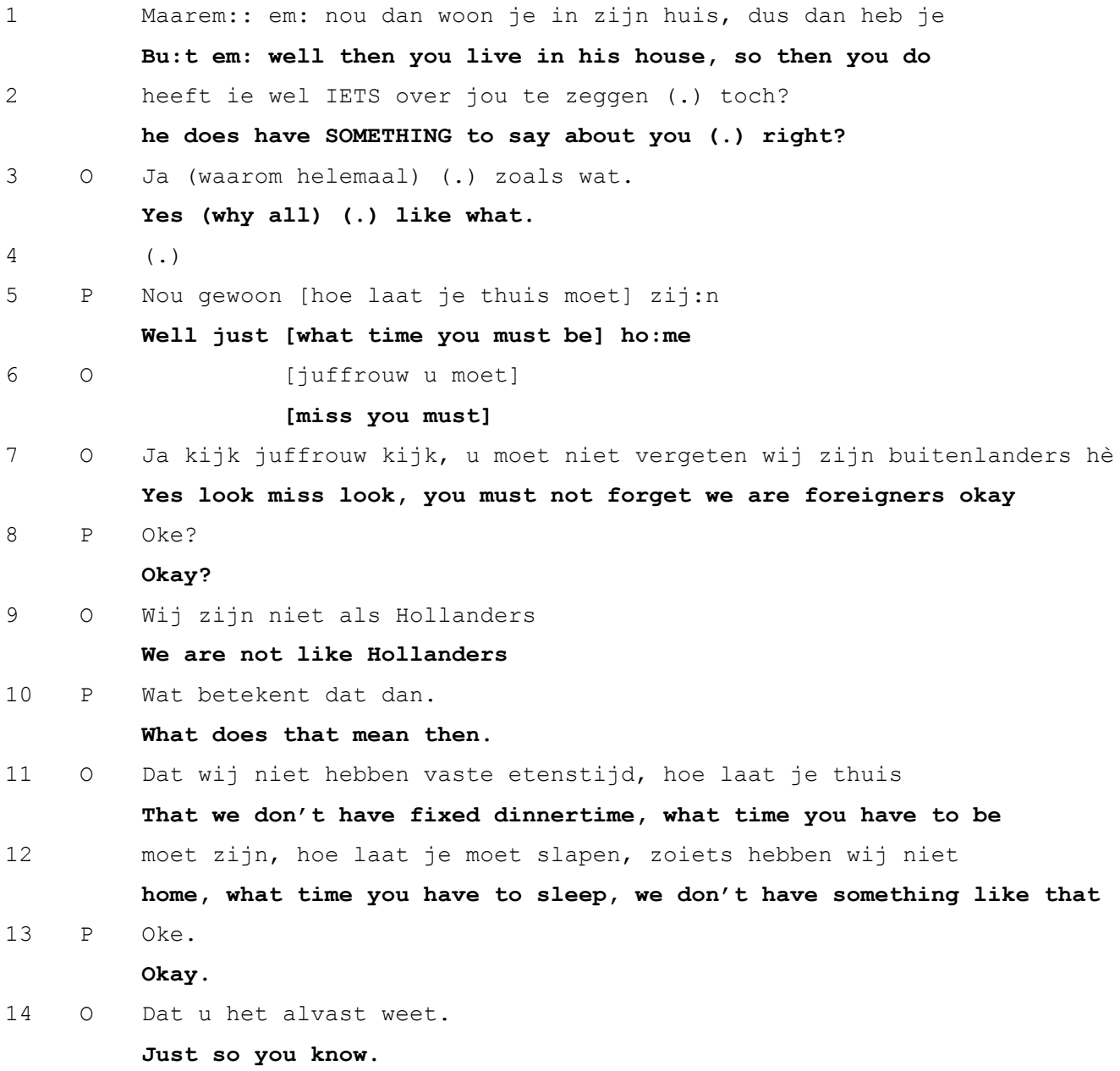


106 Nederlanders and buitenlanders

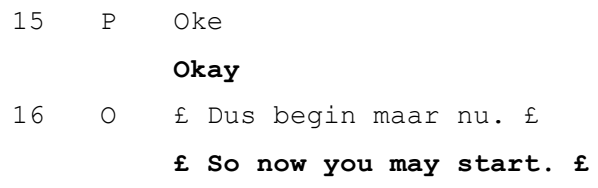

What is at play in this interaction is power, in various forms. Omer and I had different positions in the school context: although during my fieldwork I tried to mitigate hierarchy differences, I was the 'adult researcher' and he was an 'adolescent pupil.' Power relations between interactants do not only reside in institutional positions, however: they emerge in the interplay between institutional positions and speakers' "locally constructed, discursive identities" (Thornborrow 2002, 1). Just before the transcript starts, I had posed Omer a question, which marks an interactionally powerful position because it limits what can be done in the next turn. Furthermore, the question was about Omer's parents ('What do your parents think of that?'), making relevant his younger age, in which parents' opinion is deemed relevant. Omer's answer ('My father has nothing to say about me') challenges the validity of my question. In line 1 of the transcript, I cast doubt on this statement: if he lives with his father, he must have some control over Omer. This shows my orientation toward a relation between the membership categories parent and child and associated category-bound activities such as that the parent tells the child how to behave.

My turn in line 1 suggests that I doubt what Omer said, or that I am implicitly criticizing him for breaking the obligations that - to my mind result from living in a parent's house. Omer first produces a preferred response ('yes'), but then seems to change his mind and asks me 'like what.' This is produced with a dropping intonation, making it sound like a challenge (Koshik 2003). It also reverses the roles of question-asker and -answerer (Thornborrow 2002). After a short gap in line 4, which indicates some trouble probably resulting from surprise, I accept the discursive role reversal and start producing an answer to Omer's question. The somewhat extended duration of my production of the word ' $z i j n$ ' in line 5 indicates that I am starting a list of category-bound activities for the category 'parent,' i.e., 'things a parent might tell their child to do.' The first item on that list is essentially the issue Omer and I have been discussing: a parent can exert influence over when their child must be home. Omer produces a short instance of overlapping talk in line 6 which might be interpreted as 'recognitional onset' (Jefferson 1984, 12): Omer recognizes what I am trying to say and responds to it. He starts this turn before I have given my other examples, suggesting that it is not the exact content of my answer to which he objects. Rather, he seems to challenge the suggestion that I know what his situation is like because I have some 
knowledge of parent-child relationships in general. This is supported by the fact that Omer's turn in line 6 does not come at a transition-relevance place (a recognizable place where transition to a next speaker can occur, see Sacks, Schegloff, and Jefferson 1974): I had produced only one example and had not yet started to mark an end to my turn. As a result of the overlap, I end my turn.

In line 7, Omer says that I 'must not forget [they] are foreigners.' Hereby, he implicitly qualifies my previous questions and comments as irrelevant. Omer seems to say that his behavior is not a sign of a somehow 'imperfect' parent-child relationship; rather, it is explainable through their membership of the category buitenlanders 'foreigners.' $\mathrm{He}$ frames his behavior as incomprehensible to someone without his ethno-cultural background: i.e. me, the researcher. At this specific moment, in this conversation with me, he prioritizes an ethno-cultural category (buitenlander 'foreigner') over a biographic one ('son') in the characterization of the relationship between his father and himself (cf. Jaspers 2011a). Omer's introduction of this category enables him to take on the position of expert in this conversation because of his self-claimed membership of this category, casting me as an unknowing outsider. His lack of elaboration suggests that he considers this sufficiently informative for me to understand. I react in surprise in line 8, eliciting an elaboration. In line 9, Omer offers a very brief answer, again suggesting that his explicit contrasting of the categories 'foreigners' and 'Hollanders' should be enough information for me to understand (which it is not, since in line 10 I make a more specific request for an explanation of 'what that means').

In lines 11-12, Omer displays his recently acquired discursive position of expertise, listing a number of category-bound activities for 'Hollanders,' thereby constructing a contrast with 'foreigners.' Although in class $3 / 4 \mathrm{~b}$ the terms Hollander and Nederlander usually functioned as synonyms, Hollander generally has the connotation of a somehow prototypically and stereotypically 'Dutch' person, while Nederlander could be seen as more neutral. Across my data, Omer showed a predilection for using the word Hollander rather than Nederlander in many different contexts, but in this extract his choice of Hollander particularly coheres with his summing up of characteristics that in his eyes are typical for 'Dutch' people in the subsequent lines 11-12.

Lines 14 and 16 further demonstrate the shifted positions in this conversation: 'just so you know' in line 14 marks the fact that Omer (previously 'the pupil,' now 'the expert') has informed me (previously 'the adult researcher' now 'the ethno-cultural outsider') of what can be considered 'normal behavior' to whom. In line 16 , he cheekily - with a smile voice - grants me the floor. By telling me 'now you may start,' Omer retroactively changes the genre of our conversation to a formal interview-like interaction, led by him rather than 
me, although it had been a regular, informal chat as we often had. Note that throughout the interaction, Omer keeps addressing me with the formal Dutch personal pronoun $u$ (instead of informal jeljij) and the title juffrouw ('miss' or 'teacher') and is thereby, at least in form, being polite.

Throughout Example 4.1, it is visible how Omer accomplishes a shift of discursive positions with respect to his interlocutor (me, the researcher). At the start of our exchange, I was in the discursively dominant position of question-asker and adult researcher (Thornborrow 2002). It might have seemed as if I was calling into question what Omer was telling me, or that I was assessing his behavior as disobedient or disrespectful to his father and therefore objectionable. Omer changes the discursive positions of our interaction, however: he becomes the one asking questions and allocating turns. Omer's elaboration of what it means to be a buitenlander (lines 12-13) could be regarded as 'identity work' rather than as a deliberate effort to shift positions in the interaction. The idea of 'foreigners' being less restricted by their parents was not part of class 3/4b's regular discourse surrounding these categories, however, and I had not heard Omer say it before, nor did it come up later. That does not mean that what Omer claims is true or false - that is not relevant in this analysis - but it is notable that this alleged characteristic comes up when it supports his interactional move of averting critique, assuming a position of expertise, and gaining the interactional upper hand. The mention of a category, in that sense, activated the perception that those categories existed, and that they related to different customs and behaviors (as seen in Chapter 3). Being positioned as a member of a different category (as Omer did to me in the example) then can be understood to imply that it is impossible to understand one another's behavior.

We have now seen one example of an interactional move in which power positions in an interaction were negotiated by a pupil's categorization. The next interaction provides another example of such a negotiation through reference to an ethnic label that sometimes happened in class $3 / 4 \mathrm{~b}$. When conflicts occurred between teachers and pupils, and a pupil felt treated unfairly, they would sometimes accuse the teacher of treating them differently because of their membership in a certain ethnic category, that is, they would accuse the teacher of being racist. This happens in Example 4.2, which occurred at the start of a biology class. While moving from one to the next classroom between classes, Amine and Mohammed had been calling each other names and insulting each other. This started out as a playful interaction, but at the moment of the transcribed interaction, they are in the classroom and it seems to have turned into an actual (verbal) conflict. Other pupils who feature in the transcript are Stefan, Amira and Yildiz. I was sitting in the back of the classroom and did not participate in the interaction. The transcript 
starts when the pupils have sat down at their desks, the teacher wants to start the class and (again) calls the pupils to order.

Example 4.2. 10 April 2017. Biology class. Participants: Ms. Bos (B), Amine (A), Stefan (S), Amira (Ar), Mohammed (M), Yildiz (Y).

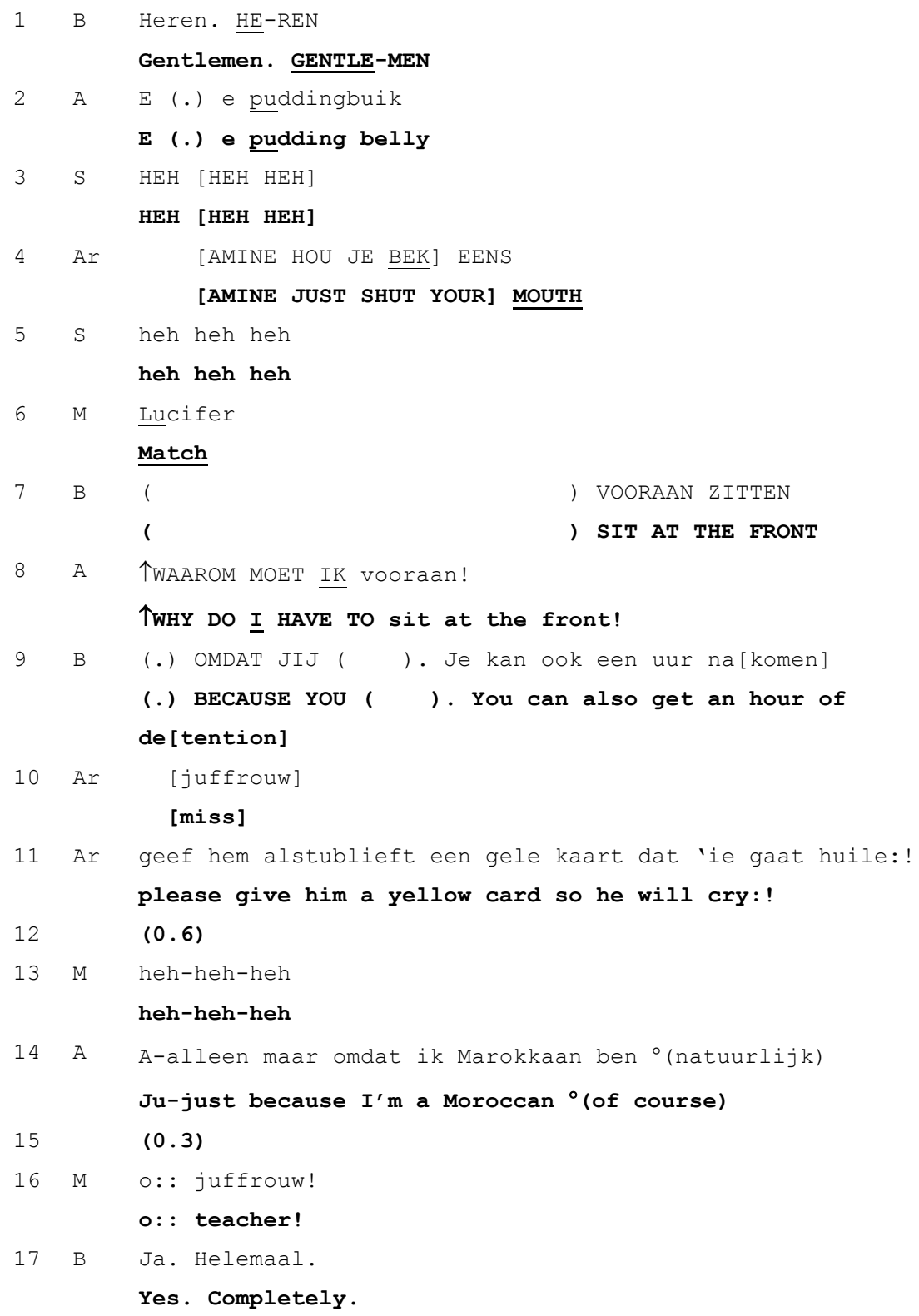




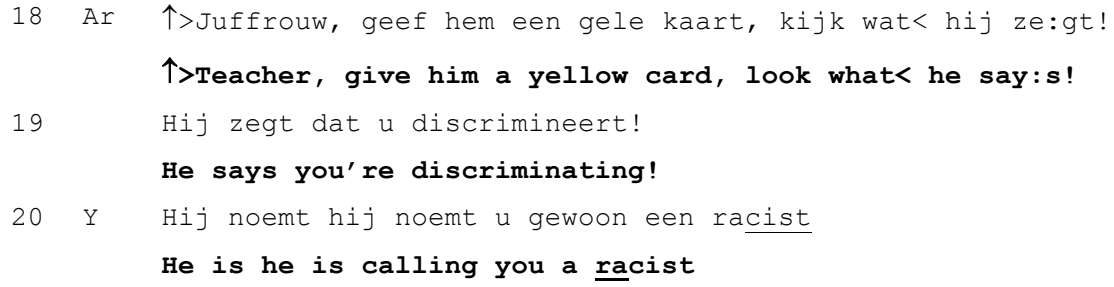

During this interaction, we can see several oppositions develop. There is an argument between Mohammed and Amine that already has been developing prior to this interaction. Furthermore, during the transcribed interaction, there is an opposition between those who are trying to put an end to this argument and those whose contributions seem aimed at making it escalate. In line 1, Ms. Bos calls out a sub-selection of the pupils with the reference term 'gentlemen.' This address term is designed to secure attention and cooperation from the pupils that are currently not giving it (Francis and Hester 2004), presumably Amine and Mohammed, who have been arguing, and potentially Stefan, who is laughing loudly. It shows Ms. Bos' intention to stop the conflict and start her class.

In an apparent effort to get the last turn in the conflict with Mohammed, however, Amine adds another insult in line 2. Amira's turn in line 4, in which she tells Amine to 'shut his mouth,' suggests that, like Ms. Bos, she wants the argument to stop. By singling out Amine, though, she can be understood to signal an understanding of Amine as the main party at fault in the conflict. Throughout the interaction between Amine and Mohammed preceding and during the transcribed interaction, Stefan has seemed most interested in making the conflict escalate. He has been shouting "oooh" when insults are made on either side, and adds loud, exaggerated laughter (as in lines 3 and 5). This seems to be designed to magnify the gravity of the comments that both parties make, and potentially to provoke retaliation by the insulted party.

Whereas Amira told Amine to shut up after his insult to Mohammed in line 2, she does not do the same after Mohammed makes a return insult to Amine in line 6. It thus seems that Mohammed has gained bystanders' support. Then, the teacher selects Amine to sit at the front row. When Amine loudly demands the reason for this in line 8, it sounds like a challenge (Koshik 2003) and shows an orientation to this punishment as unfair. Ms. Bos, in line 9 , responds, but the first part of this answer was inaudible on the recording. Amira then turns against Amine more explicitly, telling Ms. Bos to "give him a yellow card so he will cry" (lines 10-11), where 'him' and 'he' refer to Amine. A yellow card involved sending a misbehaving pupil to report to the department head, and often resulted in further disciplinary measures. The 
interaction until line 13 has put Amine in a somewhat subordinated position: the teachers as well as the pupils seem to be pitted against him. At least, he certainly seems to orient to such a position himself.

Then, in a sulky tone of voice, Amine says: "just because I'm Moroccan, of course" (line 14), dismissing the reason Ms. Bos gave in the previous turn and claiming that his category, rather than his behavior, is the reason for the 'unfair' punishment. With this, Amine conjures up a third opposition: this time, between himself and the teacher as members of different ethnic categories. Essentially, he accuses Ms. Bos of racism, as Amira and Yildiz make explicit in lines 19-20. He thereby positions himself as a victim and Ms. Bos as a perpetrator. Racism accusations are, as Augoustinos and Every (2010, 251) argue, "invariably met with not only strong denials, but also moral outrage and are often treated as more extreme than racism itself." They are likely to cause shock, insult, and anger. In line 16, the first reaction by Mohammed seems to search for such a reaction from the teacher: he makes an assessment, namely a sound of shock, followed by the address term 'teacher,' thereby selecting her as the next speaker (Sacks, Schegloff, and Jefferson 1974) in a sort of 'call to action.' Ms. Bos, however, does not react in a way that suggests shock or outrage. Her response - an upgraded agreement ("yes, completely") instead of the expectable denial (Augoustinos and Every 2010) may seem surprising. Delivered in a tone that sounds ironic, however, it does not take seriously the allegation and suggests that she is not even surprised, let alone shocked. Ironically admitting racism is thus another way in which an accused party can deny or dismiss a racism accusation. This may be available in this particular situation because the teacher has other readily available accounts for her selection of Amine for punishment.

In contrast to Ms. Bos, the other pupils do react strongly, but not in the way that Amine might have hoped. On other occasions where I witnessed pupils accusing teachers of racism, pupils did not orient to such allegations as a transgression. Often, the pupils who were members of the allegedly discriminated-against category would align with the accusing pupil against the teacher who allegedly discriminated. In this case, however, lines 16-19 show that the other pupils continue to position Amine as the party at fault in the conflict. Amira (lines 18-19) reacts to Amine's accusation in anger, orienting to it as an unforgivable transgression (Augoustinos and Every 2010; Van Dijk 1993) and again calling Ms. Bos to action to punish Amine. Amira's reaction here is striking, since a locally expectable reaction could have been for other pupils to disengage, or to align with Amine in the accusation. On the other hand, this reaction is consistent with the orientation she displayed to Amine as the party at fault in the preceding turns. In line 20, Yildiz magnifies the accusation against Amine. The statement "He is calling you a racist" suggests 


\section{Nederlanders and buitenlanders}

that Amine has not only accused Ms. Bos of engaging in a reproachable action ("discriminating," as Amira said in line 19), but that he has accused her of being a reproachable kind of person ("a racist," line 20). It seems to have the same aim as Amira's previous turn, and Stefan's earlier turns, namely, to magnify the gravity of Amine's comments and push the teacher toward disciplining him. Ms. Bos, however, does not give the girls or Amine much attention. Amine goes to sit at the front row as directed, and after about another 40 seconds of accusations between various pupils, the pupils finally open their books and Ms. Bos can start the class.

As Reyes $(2011,471)$ notes, "Crying 'racist' is not a discrete act but a discursive chain that connects across multiple domains." It occurs within a context imbued with racial and ethnic ideologies, in which marginalization of people with migration backgrounds does indeed occur. As noted previously, pupils labelled Nederlander did not often categorize other pupils as Marokkaan, Turk, or buitenlander because this could be associated with stereotyping or discrimination. This association between 'being (perceived as) a Marokkaan' and being the victim of discrimination was activated by Amine in Example 4.2.

With this analysis I do not want to deny the possibility that these pupils might actually be subjected to bias and unfair treatment from their teachers (see van den Bergh et al. 2010; Weiner 2016). It is instead to observe that, in class $3 / 4 \mathrm{~b}$, saying that a disciplinary measure was "only because I am (ethnic category)" was an action that often led to certain outcomes, and which pupils could thus recruit when they aimed for those outcomes. During my fieldwork, I witnessed several other accusations of racism against teachers. They sometimes upset the accused teacher or had the effect of getting other pupils on the accuser's side of a conflict. One time, I witnessed a pupil use it in the reversed sense when he accused the teacher of discrimination when he was told to leave the classroom: "It is only because I'm a Hollander!" (field notes, 12 January 2017). He did this in a jocular way, showing awareness of the categorical context in which such accusations did and did not make sense, and when they could be used strategically. Racism accusations could also function to gather backing from peers, and thus to strengthen the social and discursive position of the accusing pupil, converting a conflict from an individual pupil against a teacher, into one between all pupils present categorized as buitenlander against the teacher. Example 4.2 also shows, however, that racism accusations in $3 / 4 \mathrm{~b}$ were not always either met with anger or aligned with. Rather, how the teacher and other pupils would react and with whom they would side depended on the exchange preceding the accusation.

In sum, the two extracts in this section have illustrated how pupils made references to ethnic categories in processes of negotiating positions of power 
in interactions with other, locally more authoritative, actors who did not share membership in their category. In Example 4.1, Omer gained the interactional upper hand over me, the researcher, by referring to the category buitenlander 'foreigner.' He thereby implicitly categorized me as Nederlander, that is, an unknowing outsider who could not judge him, thereby achieving an 'expert' position. In Example 4.2, Amine is caught on the losing side of a conflict and makes an attempt at overturning this situation by categorizing himself as Marokkaan in an accusation of racism to the teacher. Such accusations often led other pupils to back this one pupil in their conflict with a the teacher, although in this specific interaction it did not have that effect. In the next section, I analyze another kind of interactional context in which pupils often referred to ethnic labels, that is, jocular mockery.

\subsection{Ethnic labels and jocular mockery}

People who engage with each other in a shared practice over an extended period of time, like the pupils of class $3 / 4 \mathrm{~b}$, often develop specific humorous routines. This repertoire of humorous references can be called a "joking culture,' defined as the "continued use of jocular themes by group members" (Fine and Soucey 2005, 2). Making fun of - and with - ethnic categories was an intrinsic part of the joking culture of the pupils of class $3 / 4 \mathrm{~b}$, particularly those with migration backgrounds.

Humor has been described to be an important feature of classrooms in different times and places. In his seminal classroom ethnography, Willis (1981) described the importance of the laff as the "privileged instrument of the informal" (Willis 1981,29) among a group of UK working class pupils in the 70s. The ability to make someone laugh defined who could be part of the 'in-crowd'; laughter was a temporary cure for boredom and a way to deal with adversity. Almost three decades and hundreds of kilometers away, pupils of Moroccan descent in an Antwerp secondary school engaged in a practice they called 'doing ridiculous': they play-acted building on stereotypes of the 'immigrant' and the 'local Antwerpian' to "shape and negotiate their participation at school and challenge stereotyping identity categories and elbow-room limiting situations" (Jaspers 2005a, 279). Humorous disruption can also function to set oneself apart in the group-oriented classroom setting, for example by asserting an individualized 'class clown' identity (Norrick and Klein 2008).

The kind of humor that I focus on in this chapter, i.e. jocular mockery, typically starts with a first-turn tease, for example an insult or mock threat, which is to be recognized and treated by the recipient as untrue but humorous and is intended to be appreciated by both the initiator and the recipient. When 


\section{Nederlanders and buitenlanders}

this one-turn tease develops into a longer exchange of teases and retorts, this is dubbed jocular mockery or banter (Dynel 2009). Although it is important to acknowledge the divisive potential of this (and any) kind of humor (Haugh 2010; Huuki, Manninen, and Sunnari 2010), in an extensive review of literature on teasing, Keltner and colleagues $(2001,232)$ posit that although teasing involves a degree of aggression, almost all literature reviewed recognized its function as "prosocial behavior." An example of such prosocial behavior is the establishment and reinforcement of friendship bonds (e.g. Plester and Sayers 2007). In jocular mockery, "participants are orienting to fostering solidarity, rapport or affiliation [...] within a marked non-serious or joking frame" (Haugh 2010, 2107). Much research on teasing observes this practice to be popular especially among boys (e.g. Deppermann and Schmidt 2000; Evaldsson 2005; Günthner 2011; Huuki, Manninen, and Sunnari 2010; Jonsson 2018; Møller 2017; Robles 2019). In class 3/4b, jocular mockery was a popular interactional style also among girls.

In Example 4.3, Amira, Yildiz, Dounia and Meryem explicitly construct jocular mockery as a category-bound activity, identifying it as one of the main differences between their locally drawn category buitenlanders, who did engage in this activity, as opposed to tatta's (i.e. Nederlanders), who did not. This interaction occurred during a group discussion with Amira (who labelled herself Marokkaan), Yildiz (who labelled herself Turk), Dounia (who labelled herself Marokkaan), Meryem (who labelled herself Turk), and the researcher (who was labelled as Nederlander by the pupils). The pupils have been discussing what they perceive to be typical characteristics of tatta's, when I pose the question in line 1 of the transcript.

Example 4.3. 16 June 2017. Group discussion. Participants: Amira (A), Yildiz (Y), Dounia (D), Meryem (M), and the researcher (Pomme, $P$ in the transcript).

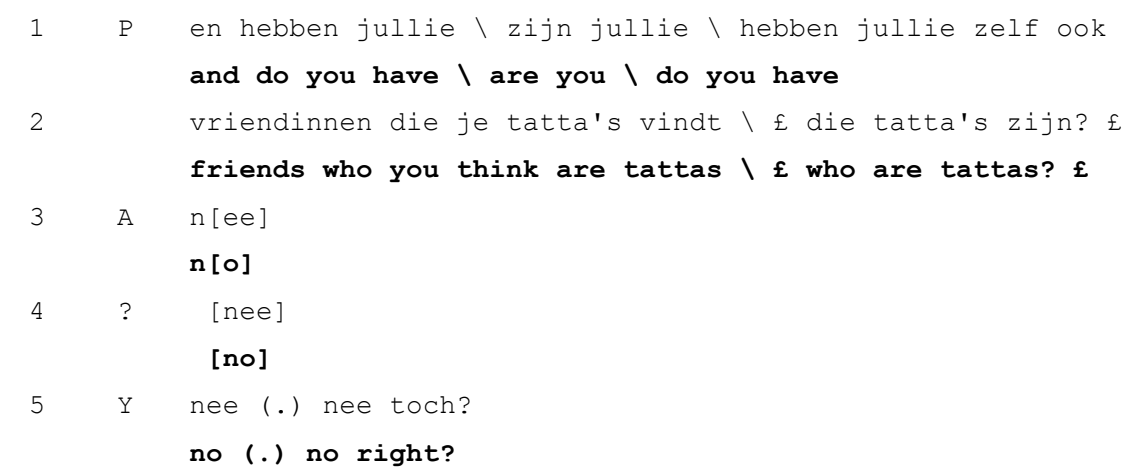




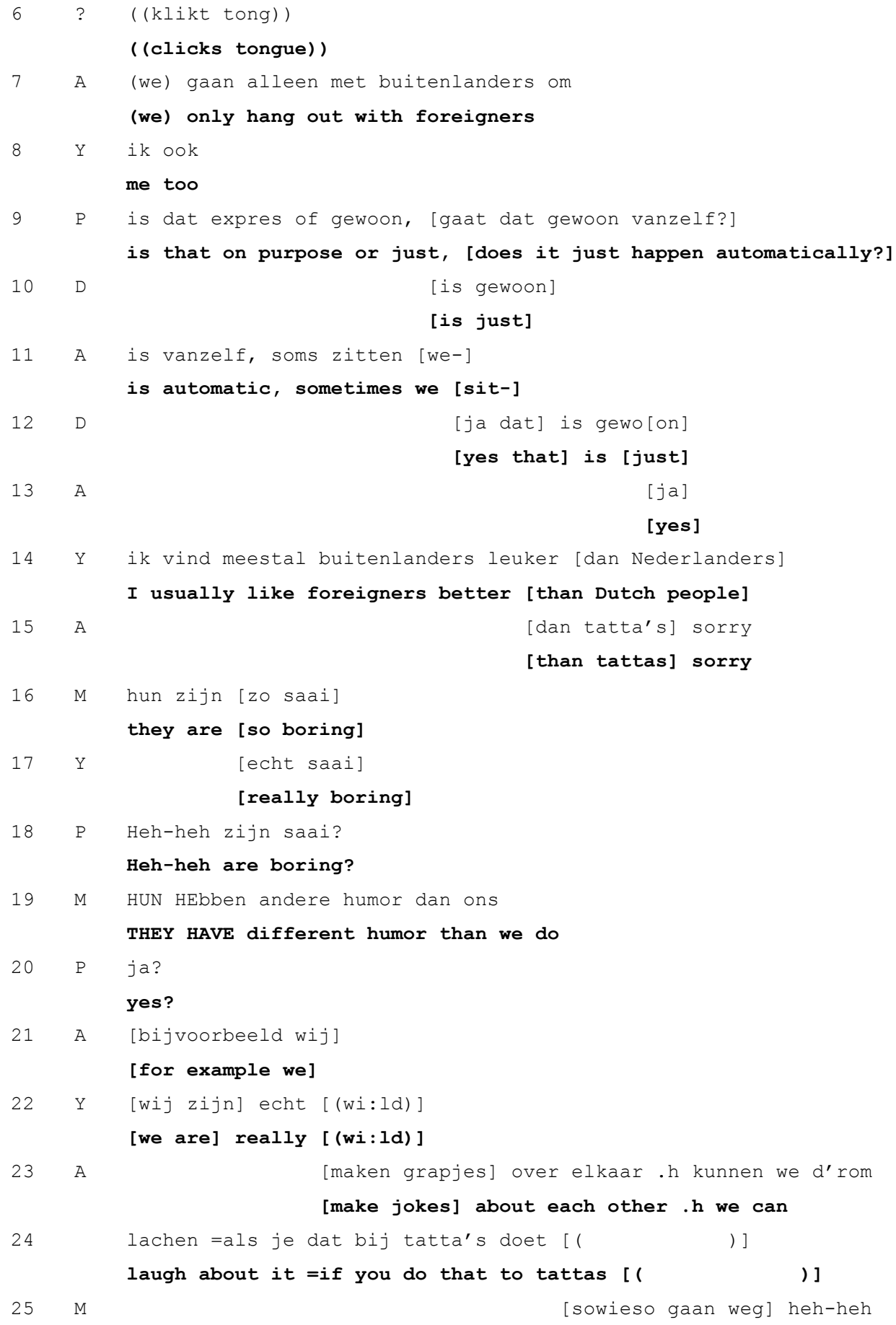




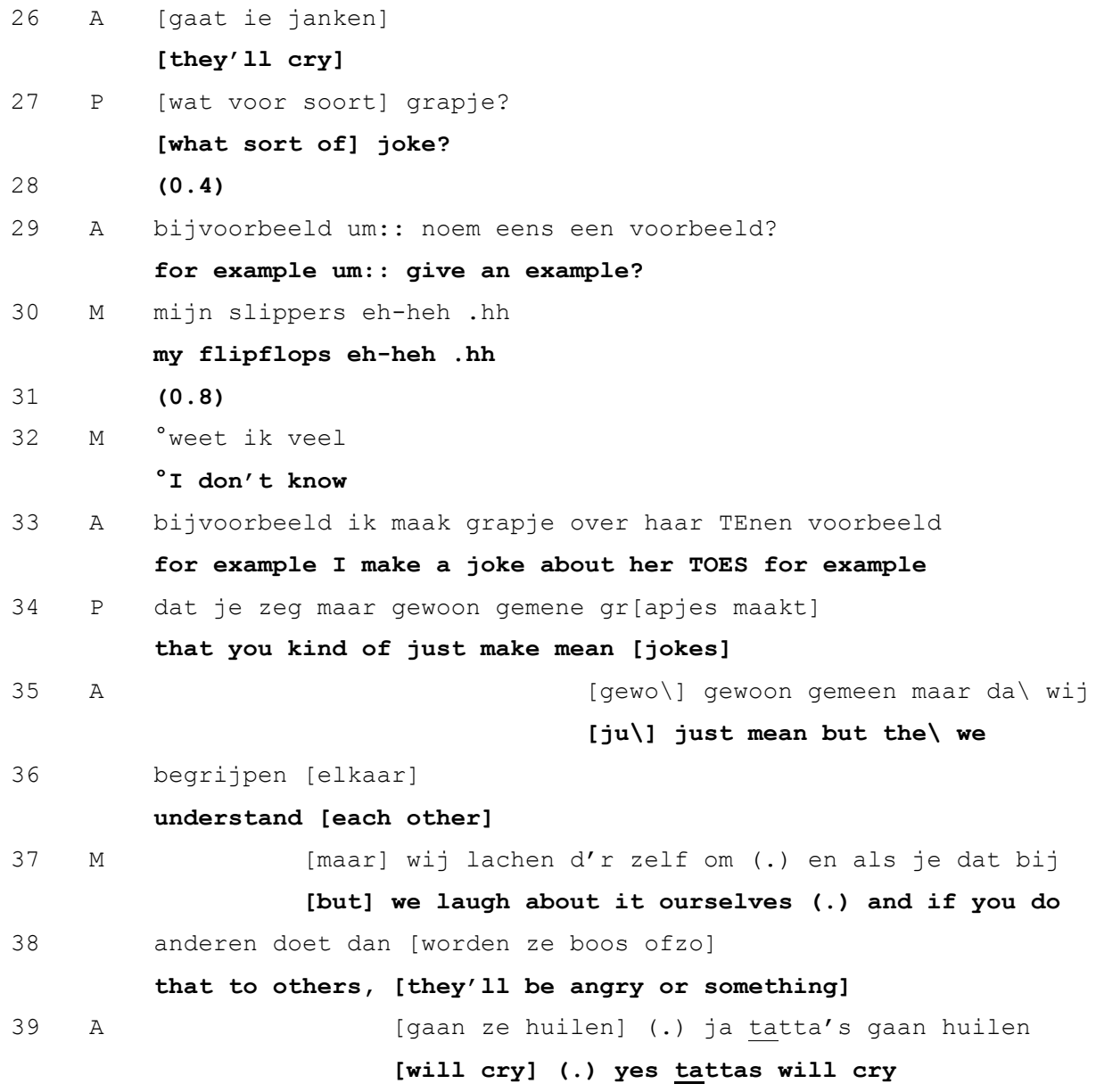

In lines 1-2 I ask the girls whether they have 'friends who are tatta's' (a synonym of Nederlander). This was the term that the girls and I had already been using in the conversation preceding the transcribed interaction (transcribed in Example 3.11 in Chapter 3). They had used tatta to categorize other people in opposition to themselves as Marokkaan, Turk or buitenlander, and I follow this categorization in the interaction. In line 7, Amira makes relevant the 'opposite' category to tatta, which was implicit in my question, that is, the category buitenlanders 'foreigners.' In line 9, I probe them to tell me why they only hang out with 'foreigners.' The girls account for only having 'foreign' friends by referring to category predicates that, in their eyes, are tied to being a buitenlander or a Nederlander from line 10: buitenlanders are more likeable, whereas Nederlanders are boring. Yildiz's switch from the term tatta, 
which is informal and potentially mildly derisive, to the more 'neutral' Nederlander may show her orientation to me, a researcher who might prefer more formal terms and whom they had furthermore categorized as Nederlander. This may also explain Amira's 'sorry' in line 15, although this sounded more defensive and humorous than apologetic. My laughter and repetition of the words "are boring" with questioning intonation, in line 18, indicate that I have not taken offense, but that I want to know more.

Then, from line 19, the girls explain the difference between buitenlanders and tatta's as mainly a matter of sense of humor. Dounia, Amira and Meryem build this argument together: they add overlapping turns, building on what the previous person said. Amira explains that they make jokes about each other (lines 23-24), but that if you do that to tatta's, they will not appreciate it. In line 34, I call this kind of humor 'mean jokes,' but in other words, they seem to be referring to the practice of jocular mockery. In lines 37-39, the girls allude to the function of this kind of humor: they understand each other, and it makes them laugh. In my observations of these girls, jocular mockery indeed seemed to be an important way in which they reinforced their friendship bonds.

The example that they give - laughing about Meryem's toes in her flipflops - has little to do with ethnic categories. Many of the jocular mocking interactions that I observed did not draw my attention because they were especially insulting, however, or because they were done only by pupils who categorized themselves as buitenlander. Rather, they caught my interest because so many of them involved humor about ethnic categories, and because so many of all the ethnic category references that I observed and coded occurred in jocular mocking interactions (to be precise, 83 out of 265 , that is nearly one-third). Jocular mockery and ethnic labels seemed intimately connected. The exchange between Amira and Yildiz in the opening vignette of this chapter is one example of this. The primary function of it did not appear to be mere categorization of the self or someone else as an incumbent of an ethnic category, but rather to engage in this locally established practice, their 'joking culture' that, among other things, served to emphasize friendship bonds (Fine and Soucey 2005).

The specific type of humor that deals with ethnic categories, in class $3 / 4 \mathrm{~b}$, seemed to do more than just emphasizing who was 'in' and who was 'out,' though. Jokes have a potential to have fun and break tedious school routines, but also to undermine racist voices (Jonsson 2018). As Woolard (2018) argues, humor can be a way to resist marginalization, particularly for minoritized speakers. It can allow them to "acknowledge, reproduce, and challenge ethnolinguistic stratification" (Da Silva 2015, 206); it can be ambiguous and reflexive; it can challenge as well as reinscribe dominant 
discourses (Halonen and Pietikäinen 2017). Winkler Reid (2015) describes practices of humor around ethnicity and race in a UK classroom, arguing that pupils used it to downplay hurtful racist discourses that exist in society. For those pupils, laughing about their own and other people's categories served the function of "creating alternative relations that cut across notions of sameness and difference" (Winkler Reid 2015, 33; see also Rampton 2005). While humor about race and ethnicity did not blur boundaries in that classroom, it made them into a 'laughable' (Ford and Fox 2011) and thereby enabled pupils to deal with potentially divisive differences. As Leveen (1996) notes, laughing together about what in actuality might be experienced as offensive and painful can be a way to mitigate the effects of exclusionary practices and stigmatization. In class $3 / 4 b$, jocular mockery with ethnic labels did not blur boundaries between categories nor made them a 'laughable': these categories were very real to the pupils. The laughable, in their case, seemed to reside in the discourse about the categories. Pupils showed keen awareness of the disadvantaged position they occupied because of their migration backgrounds, and they seemed to address this through humor.

Example 4.4 shows a typical interaction of jocular mockery with ethnic categories in class 3/4b. Two pupils, Khadija and Amira (both categorized themselves as Marokkaan) engaged in a short exchange of mock insults and retorts during a Care and Well-being class. Amira was practicing hairdressing on a mannequin and at the start of the transcript (in line 1) complains that the mannequin is in a bad state. I was sitting at the same table where Amira had her mannequin installed. During the transcribed interaction, Khadija passed Amira's workplace and commented on what Amira was doing. Amira and Khadija had been classmates for a few years, but I never saw them sit together in classes or breaks and would not characterize them as close friends.

Example 4.4. 19 May 2017. Care and Well-being. Participants: Amira (A), Khadija (K), Researcher (Pomme, $\mathrm{P}$ in the transcript).

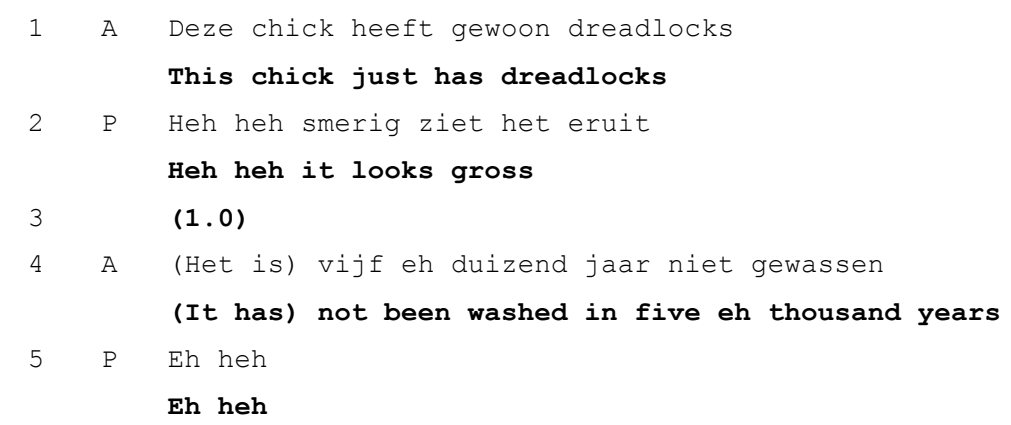




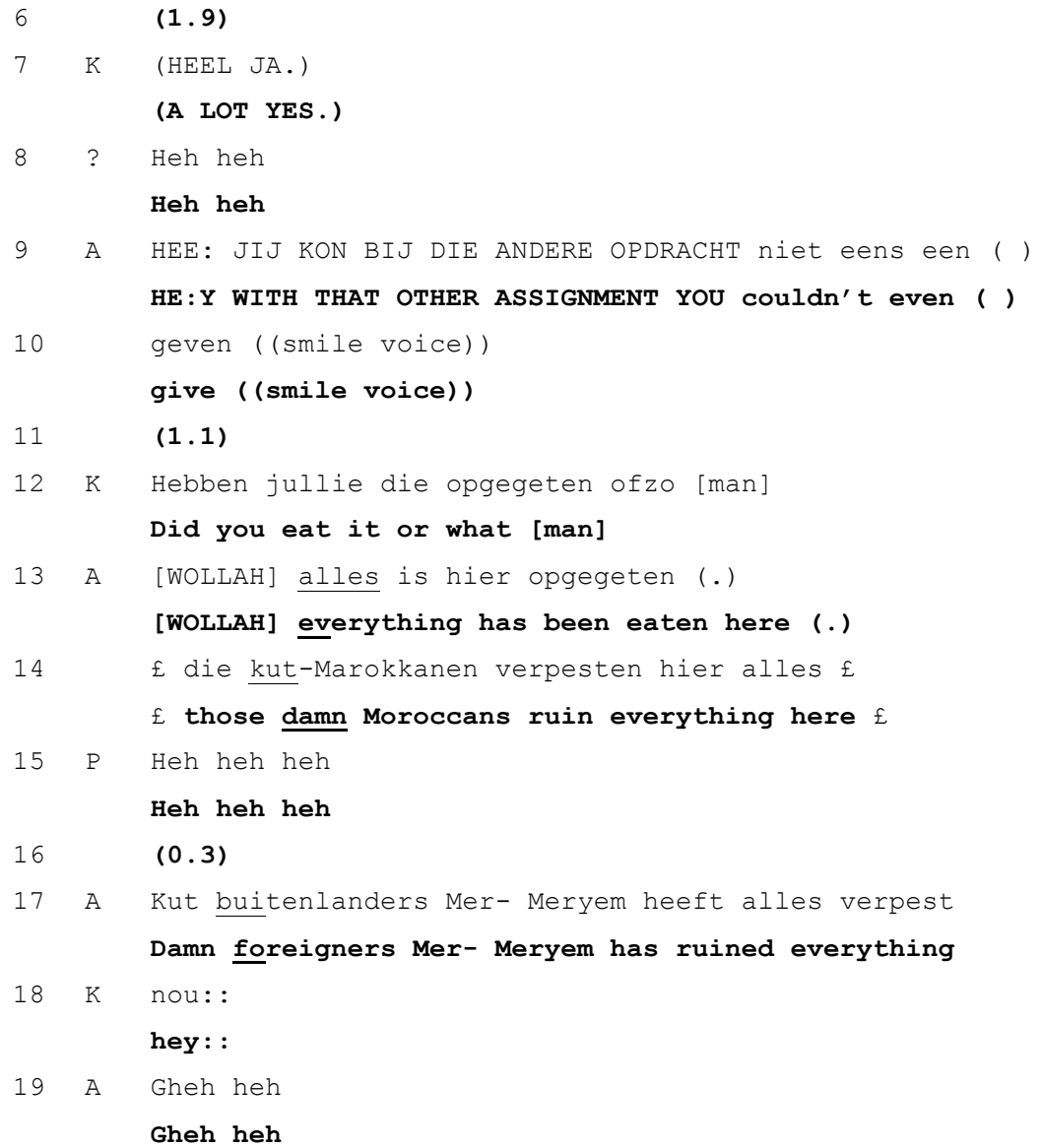

Gheh heh

In line 1, Amira comments on the mannequin she is using to practice hairdressing. As a result of its intensive use, its hair is hard to practice hairdressing on: she refers to the mannequin's hair as 'dreadlocks.' Amira sounds mildly ironic and clearly irritated with the mannequin. Being the only person in her vicinity at that moment, I felt compelled to react to her frustration, and in line $2 \mathrm{I}$ laugh and align with her statement. The rather long gap in line 3 (like the other gaps between turns in lines 6, 11 and 16) stems from Amira's simultaneous engagement in her assignment: she did not seem to be trying to start a conversation but appears to be engaged in self-talk, distracting herself without expecting much reaction. In line 4, Amira makes an extreme case formulation ('it hasn't been washed in five thousand years'), with which she frames her comments as jocular and displays reduced investment in what she is saying (Haugh 2010; Edwards 2000). During the pause in line 6, Khadija passes by Amira's table and makes a loud statement 


\section{Nederlanders and buitenlanders}

(line 7). The exact content of what she says is unclear, but her intonation drops at the end, and it sounds as if she has somehow negatively assessed Amira's work with the mannequin. This can be identified as the first-turn tease that sets off a banter exchange between the girls (Dynel 2009).

In line 8, the first reaction by another classmate is laughter. This laughter sounds affiliative, indicating that this classmate interpreted the comment as jocular. Amira replies in line 9 with a retort, following the conventions of ritual-insult: she quickly responds to Khadija, trying to outtalk or out-perform her (Evaldsson 2005; Goodwin 2002). It is uttered in a smile voice, indicating the playful character of her utterance (Haugh 2010) and inviting laughter (Jefferson 1979). Khadija's lack of immediate response - she does not laugh nor say anything back - leaves room for interpretation, but the researcher's familiarity with this peer-group implies that she would have responded immediately if she had felt insulted.

In line 12, Khadija continues the ritual insulting sequence, asking Amira whether 'she has eaten it.' 'It' appears to refer to the mannequin or its hair, and the rather absurd question continues the jocular frame in which the pupils have entered. Amira responds by aligning with Khadija in another jocular (and absurd) extreme case formulation, stating that 'everything has been eaten here.' This shifts the frame from personal insults directed at each other, to the safer topic of their shared environment: the classroom. By 'here,' she seems to complain about the supposed bad state of 'everything' in the classroom. This upshot does not spark a reaction by Khadija. Until this moment, a jocular frame has been established between the girls, but no references have been made to any labels.

Then, in line 14, Amira magnifies as well as specifies her extreme case formulation of line 13: she now attributes blame to die kut-Marokkanen 'those damn Moroccans' for ruining 'everything.' Amira evokes a very specific discourse in the Netherlands with her use of the term 'kut-Marokkanen,' which, literally, means 'cunt Moroccans.' In 2002, a Dutch politician was (inadvertently) recorded using this term, and it has since become part of a vocabulary that puts forward an image of Moroccan-Dutch youth as troublesome, 'un-integrated' and criminal (De Koning 2016). Amira's use of the term 'kut-Marokkanen' indicates her awareness of, and ridicules, that discourse. Her statement in line 14 follows a pattern of jocular mockery which occasionally occurred in class $3 / 4 \mathrm{~b}$, where any negative situation could arbitrarily be attributed to 'those (damn) Moroccans' or 'those foreigners.' In an instance of vari-directional double-voicing (Bakhtin 1984 in Rampton 2005) or 'say foring' (Jaspers 2005a, 284), Amira uses the voice of someone else whose view is opposed to her own, echoing racist voices in Dutch society that blame Marokkanen for any negative situation. She does this without signs 
of stylization: she does not draw on linguistic markers associated with, for example, the category Nederlander, to make her utterance stand out as a humorous imitation (cf. Da Silva 2015; Halonen and Pietikäinen 2017; Jaspers 2011b). The content is enough for the rest of the interactants to recognize it as such, however. In lines 15 to 19 , the researcher reacts with laughter, Amira continues with some jocular mocking statements about buitenlanders and about her friend Meryem (who was not participating in her activity or the conversation), while Khadija has already moved on to other business. Khadija's lack of reaction suggests that she is not offended, nor surprised at this comment from Amira.

The interaction in Example 4.4 can be characterized as an interactionally cooperative or 'successful' instance of jocular mockery: the girls achieve alignment and orient to the other's expressions in a way that was accepted and expected in this context. Pupils used humor to temporarily interrupt the serious and boring business of doing school assignments (Jaspers 2005a; Willis 1981). Amira and Khadija were not close friends, but they could nevertheless build on a joking culture that was familiar and well-established in this context: humor with ethnic labels. Amira's introduction of the label Marokkaan within a jocular mocking context framed the interaction between herself and Khadija as one between members of the same category making fun, rather than as two classmates criticizing each other. As such, she safeguarded relations between herself and Khadija. These pupils' particular joking culture served more serious functions than just 'having fun,' however. Laughing about stigmatizing, exclusionary, and hurtful discourses also mitigated their effects (Leveen 1996). Crucially, only pupils who labelled themselves as Marokkaan, Turk or buitenlander would regularly engage in jocular mockery with labels. In the next section, I examine what happened on the rare occasions in which pupils labelled Nederlander attempted jocular mockery with ethnic labels.

\subsection{Mockery by a non-member of the mocked category}

In class $3 / 4 b$, jocular mockery that built on the topic of descent, race or ethnicity functioned to have fun, undermine stigmatizing discourses and construct category-based solidarity. Doing such interactional work with this kind of humor was not openly available to anyone in the class, however. As is the case with other actions involving the use of ethnic or racial categories (e.g. Whitehead 2018), 'ethnic' humor runs the risk of being interpreted by interlocutors as transgressive of social conventions, that is, as racist, especially when performed by people who are not categorizable as member of the mocked category. Jocular mockery that targets the self or the 'own group' has been observed to be more easily acceptable than that which targets a racialized 
Other, as it functions on the ideological assumption that "the use of ethnic slurs is acceptable as long as the speaker can claim membership in the specific ethnic group labelled by the slur" (Chun 2004).

In class $3 / 4 \mathrm{~b}$, the social restrictions surrounding who could make use of jocular mockery with ethnic references meant that pupils considered a 'minority' (a buitenlander in their own terms) could joke about members of the category Nederlander, but also about other 'minorities' even if they did not share membership in the exact same category (see also Møller 2017). It was common for pupils labelled Turk to joke about the category Marokkaan or vice versa, especially (but not only) if the joke initiator and the butt of the joke were friends. This suggests that in such instances, the category that was treated as relevant for the purposes of the action at hand was not always the one that was explicitly mentioned. That is, even if pupils mentioned the category 'Moroccan' in their joke, the category that enabled them to make this joke appeared to be the 'foreigner' category.

Jocular mockery about ethnic categories was usually off bounds for members of the category Nederlanders. Whereas pupils labelled as Marokkaan, Turk, or buitenlander were seen as 'imitating' rather than 'doing' stigmatization of members of their own category, since they were also victimized by it (cf. Sacks in Schegloff 2007), pupils categorized as Nederlanders had to be careful not to be perceived as 'doing' that discourse. Pupils' own perceived categories thus restricted the interactional moves that they could accomplish with references to labels. Example 4.5 and 4.6 show a key incident during my time at South High School, during which those restrictions came to the fore due to being breached. The interaction occurred while a group of pupils was sitting at a large table together, working on book assignments. Stefan (16, who categorized himself as Nederlander), Ben (17, who categorized himself as Nederlander), Omer (17, who categorized himself as Turk), and Meryem (16, who categorized herself as Turk) were sitting at one table. I was sitting with them. At the adjacent computer table were Jessica (16), Naomi (16), and Nikki (15), who were all categorized as Nederlanders. On the other side of the classroom was Khadija (16, who categorized herself as Marokkaan). For a few days already, the pupils have been harassing Stefan about his hair: they have been saying it is too long and he needs a haircut. At the beginning of the transcription, Ben starts talking about this again.

I present this interaction in two parts: Example 4.5 and Example 4.6. In Example 4.5, the mockery that occurs is hardly characterizable as jocular. The pupils are making fun of Stefan, and his reaction consists of trying to defend himself instead of laughing or making return insults. Example 4.5 is an important backdrop to what occurs later in the interaction in Example 4.6, in 
which a non-member of the mocked category intents to engage in categorical jocular mockery.

Example 4.5. 6 November 2017. Care and Well-being. Participants: Ben (B), Jessica (J), Omer (O), Meryem (M), Naomi (N), Stefan (S), and the researcher (Pomme, $\mathrm{P}$ in the interaction).

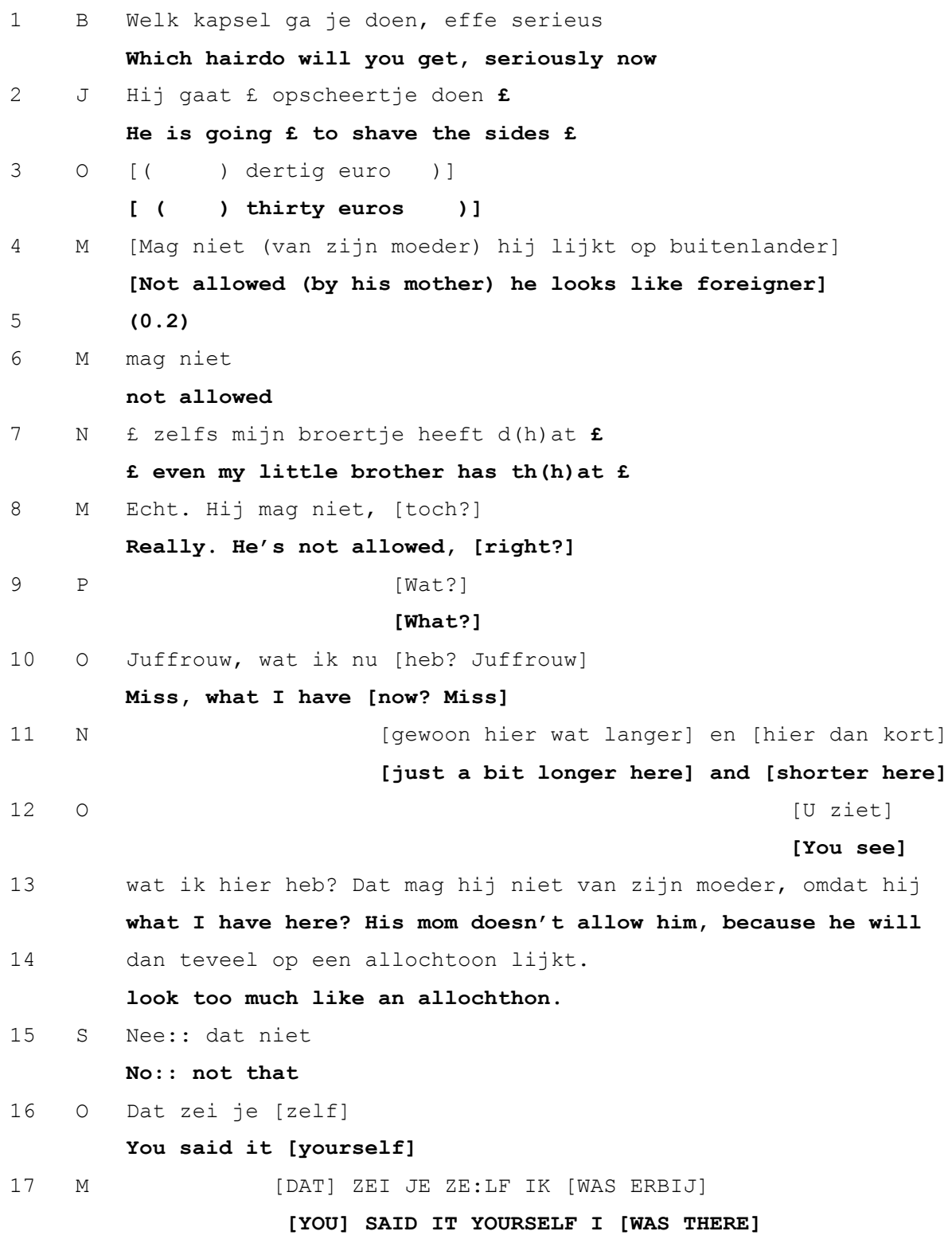


124 Nederlanders and buitenlanders

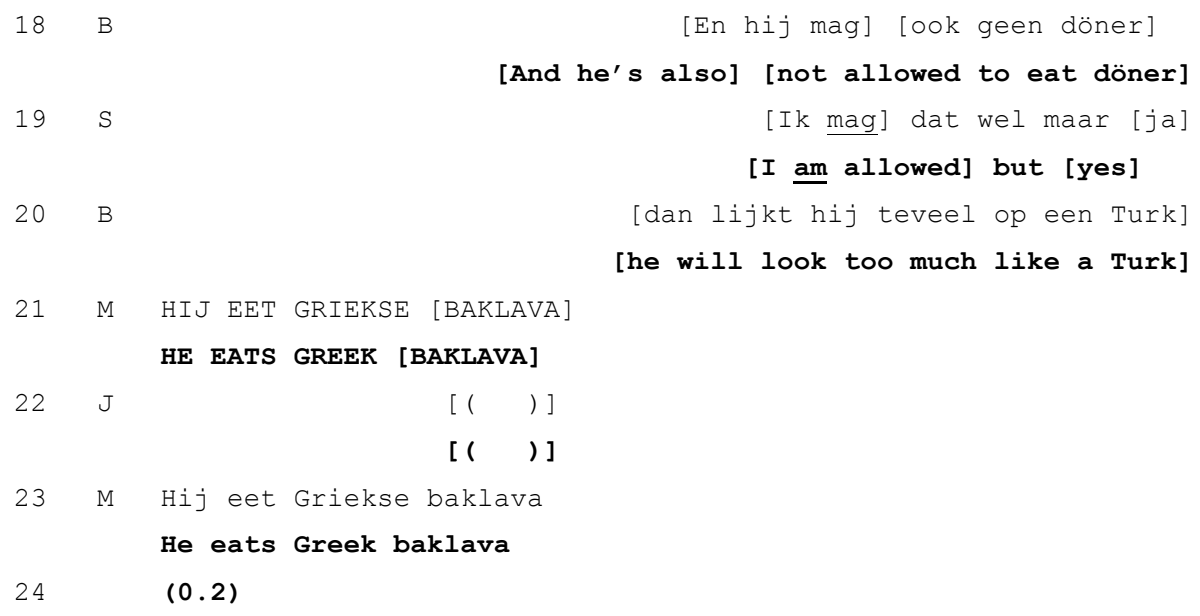

The interaction starts when Ben asks Stefan what hairdo he will get. This keys an interaction in which the rest of the pupils build on prior interactions to pick on Stefan. Stefan occupied a difficult position in class 3/4b: he was not very popular and usually quieter than the rest. Although Stefan is explicitly selected as next speaker, in line 2 Jessica provides the second pair-part to Ben's question (Lerner 2019). Her comment that 'he will shave the sides,' referring to a hairstyle that was popular among young men around this time, is delivered in a smile voice that indicates that this is not a serious answer. It follows Ben's keying to mock Stefan. This sets off a fast-paced and animated exchange among the pupils consisting of much overlapping talk. In lines 4-6, Meryem repeats what Stefan allegedly said in an earlier conversation: that he is not allowed to get that haircut as it would make him 'look like a foreigner.' Naomi states that 'even' her baby brother has that haircut, framing the idea of the relation between a haircut and looking like a 'foreigner' as senseless. This can be understood as alignment with the pupils mocking Stefan by mocking what his mother supposedly said. All this time, Stefan himself does not produce the second part of the question-answer adjacency pair.

Meryem's turn in line 8 is directed at me. Her repetition of Hij mag niet 'He's not allowed,' followed by the tag question toch? 'right?,' appears to be a request to me to confirm what Stefan said. It seemed like I was being recruited in mocking Stefan. However, I had not been present for the earlier conversation, and I did not want to align with the pupils against Stefan. My open-class repair initiator Wat? 'What?' in line 9 was an attempt to comply, though minimally, with her selection of me as the next speaker, without specifying the source of trouble. Omer and Naomi take this up it as a problem of understanding rather than hearing and proceed to fill me in on what 
allegedly happened in lines 10-14. Omer makes Stefan's comment personal by pointing to his own haircut ('You see what I have here?' line 12-13): he actually has the hairdo the pupils are talking about, and during my fieldwork he regularly categorized himself as buitenlander or Turk. In line 14, Omer replaces the earlier-used term buitenlander for allocbtoon ('allochthone'), which used to be the 'official' Dutch term for people with a migration background. This word was abolished from official usage in 2016 as it had become associated with stigmatizing images, and its pejorative use had become widespread (for an analysis of this term, see Yanow and van der Haar 2013). It is still sometimes used in popular discourse, but in class $3 / 4 \mathrm{~b}$, I only heard Omer use it a few times. In this particular instance, he might have used it because he orients it to me - an outsider to the peer-group, and someone who he might think prefers 'formal' terms. Omer says that Stefan's mother does not allow Stefan to get this haircut, implying that (in Stefan's mother's eyes) it would be undesirable to look like an 'allochtoon.'

In line 15, Stefan makes his first contribution to this conversation about him, but Omer and Meryem directly override him in lines 16 and 17. Stefan struggles during this whole exchange: the four times he tries to say something (lines 14 and 18 in this part, and 27-28 and 30 in its continuation in Example 4.7), he reacts seriously to his classmates' teasing, trying to set the record straight (cf. Robles 2019). He does not laugh with them, or try to out-perform them, as commonly happened in successful and affiliative sequences of jocular mockery (Billig 2005; Haugh 2010). Furthermore, he is trying to set the record straight as to whether his mother does or does not allow him to do certain things (line 19, 'I am allowed but yes'), orienting his response to the suggestion that his mother dictates his hairstyle. He does not address the issue of whether she (and he himself) thinks that a hairstyle is indexical of a membership category or whether being/appearing to be member of that category is desirable. Stefan does not achieve interactional alignment with his peers.

In lines 18 to 23, several of the pupils produce overlapping turns, moving the topic of discussion from Stefan's hairstyle to certain dishes. Different interesting observations could be made about these lines, but for the purpose of the argument, I will focus on what happens in the next part of the interaction, transcribed in Example 4.6. In this part, Nikki (who was categorized as Nederlander) makes her attempt at jocular mockery with an ethnic label. 


\section{Example 4.6. Continuation of Example 4.5.}

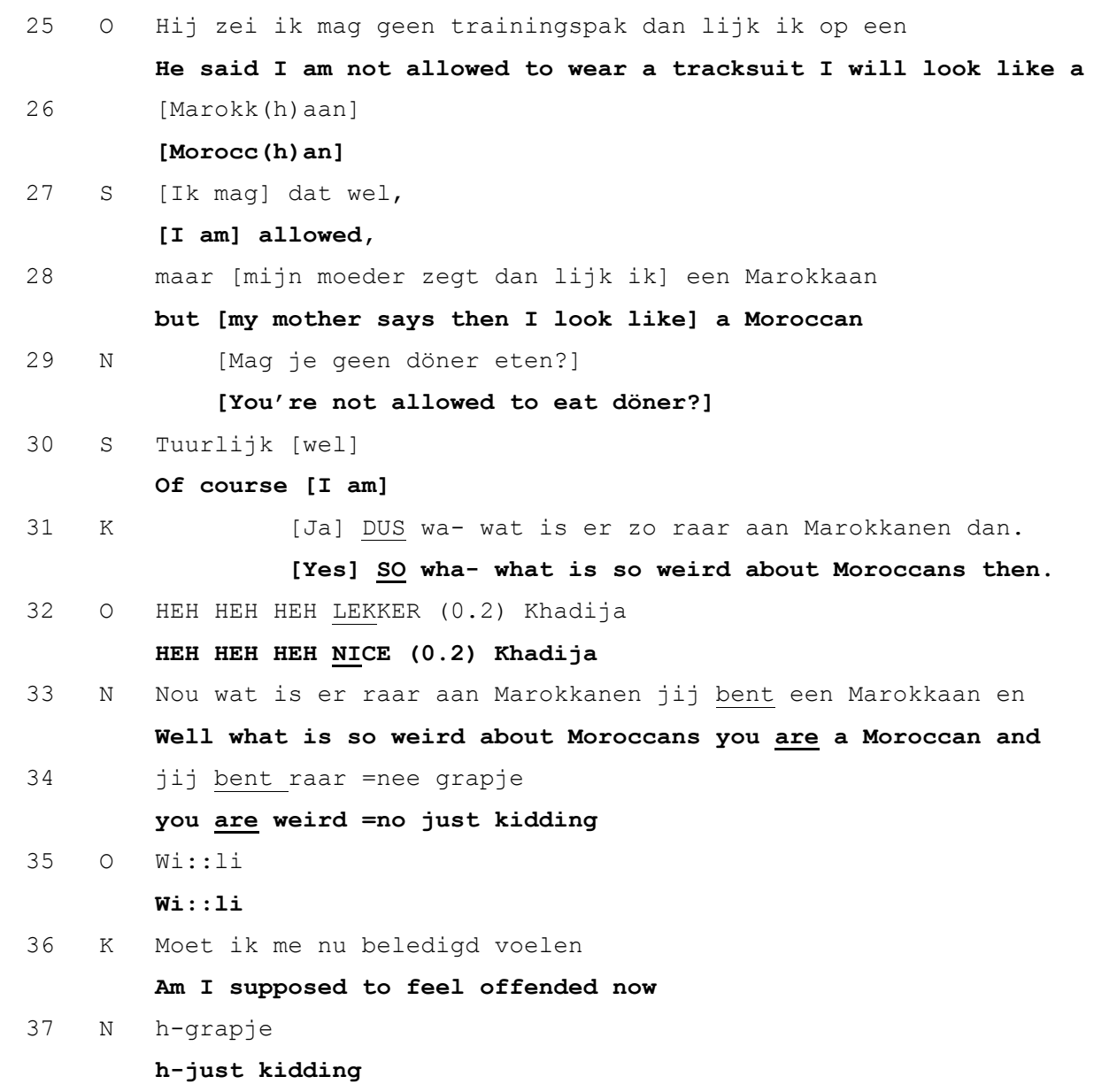

After having used the labels buitenlander (line 4), allochtoon (line 14), and Turk (line 20), Omer introduces the label Marokkaan into the conversation in line 26. This new label does not change the content of the preceding conversation, but it does seem to be what sparks Khadija's entrance into the discussion in line 31. (Lines 29 and 30 are in response to Ben's comment about eating 'döner' (kebab) in line 18 of Example 4.5). During my fieldwork, Khadija regularly categorized herself as Marokkaan. During this interaction, she was sitting alone at the other side of the classroom and had not been participating. It seems that she treats Stefan's mention of the label Marokkaan in line 28 as an attack and responds. Khadija's turn in line 31 is phrased as a question to Stefan to say what is so 'weird' about 'Moroccans,' but its intonation - 
dropping at the end - makes it sound like a challenge (Koshik 2003). The response options for Stefan are limited here. In the face of this challenge, he could either back down (e.g. apologize) or pursue his point (cf. Whitehead, Bowman, and Raymond 2018), but none of these seem particularly tempting in the face of this interaction, and Stefan does not respond.

But the question is still 'dangling,' since a second pair part has not been delivered. In line 33, Nikki provides the answer to the adjacency pair initiated by Khadija. Her comment ('Well what is so weird about Moroccans you are a Moroccan ...') seems to be designed to tap into the resource of jocular mockery with reference to ethnic labels described in the previous paragraph. This would function to release tension by instantiating laughter. A relevant and preferred response to joking is laughter (Sacks in Haugh 2016, 128), usually initiated during the joke turn. Another potential preferred response, if the turn was taken as the initiation of a jocular mocking exchange, would be an immediate return insult (as seen previously in Example 4.4, see also Haugh 2010). Nikki's turn does not spark any of these reactions, however: during and immediately after the turn, the other pupils remain silent, and Khadija does not come with a retort. The expression "just kidding," which Nikki adds right away, "does not simply pre-empt offence, but represents a deeply moral claim through which participants also acknowledge possible offence" (Haugh 2016, 129). In other words, Nikki seems to have noticed that she is not getting the intended reactions and may have realized that she has said something potentially offensive.

The first response to Nikki's attempt at jocular mockery is by Omer, who says 'wi::li' (line 35) - an exclamation associated with Arabic, used to express surprise, shame, or lament - something like 'wow.' He hereby confirms the 'need' for Nikki's disclaimer, but orients to its failure to preempt offense. His choice of an Arabic expression to do so might be interpreted as emphasizing the opposition between buitenlanders and Nederlanders that has been relevant in the preceding conversation. Khadija's reaction in line 36 shows no signs of appreciation of Nikki's joke; 'am I supposed to feel offended' is said with dropping intonation and without signs of laughter or smile voice. Once again, Nikki states that she was only joking, but no one responds, and the pupils fall silent.

The poor reception of this attempt at jocular mockery can be explained by various contextual factors. In the interaction that started in Example 4.5, label references functioned somewhat differently from those in the previous extracts. In previous extracts in this chapter (Example 4.1, 4.2, and 4.4), labels were used to achieve interactional moves: Omer managed to take the upper hand in conversation by using an ethnic label; and Khadija and Amira built on a routine of jocular mockery with ethnic labels. At the start of Example 
4.5 , however, the discussion is about the sociocultural content of the categories themselves, that is, about the pairing of membership categories with categoryincumbent features (Sacks 1995). In that sense, this first part of the interaction is similar to the interactions that I analyzed in Chapter 3, in that it contains pupils' 'meta-categorical' negotiations about which characteristics are indicative of category membership (in this case, the relation between a haircut, clothing style, and food, and being perceived as a category member), and whether being perceived as a member of that category is negative. Nikki, however, seemingly tries to use labels with an interactional purpose, that is, within jocular mockery to interrupt serious business and cause laughter. She fails not only because she breached the restrictions surrounding which pupils could make use of labels as interactional resources (that is, not Nederlanders), but also because she produced her turn within a conversation that was already dealing with sensitive issues.

The last interaction of this chapter supports the proposition that in class $3 / 4 \mathrm{~b}$, the most important factor in determining who could engage in jocular mockery of membership categories of which they themselves were not a member was the joker's own perceived category. In Example 4.7, a pupil labelled Nederlander makes a jocular mocking comment about Turken within a context of lighthearted jocular mockery surrounding ethnic categories. The comment is carefully designed, but its reception is nevertheless ambiguous. This interaction occurred toward the end of a Care and Well-Being class. The teacher, Ms. Willems (who was categorized by the pupils as Nederlander), was sitting behind a desk on the side of the classroom and I was sitting next to her. Ms. Willems was telling me about her plans for the summer holidays. On the other side of the desk were some pupils, including Ben (who categorized himself as Nederlander), Omer (who categorized himself as Turk), and Stefan (who was categorized as Nederlander). The class was nearing its end and the pupils were hanging around, waiting for the bell to mark the end of class. The transcribed interaction starts when Omer overhears Ms. Willems and me talking about her holiday plans.

Example 4.7. 6 November 2017. Care and Well-being. Participants: Omer (O), Ms. Willems (W), Stefan (S), Ben (B), and the researcher (Pomme, P in the transcript).

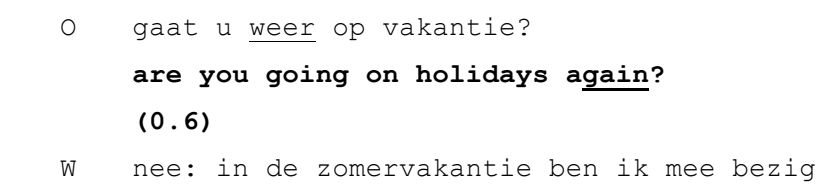


no: in the summer holidays I am busy with

(0.7)

nu al

now already

=nu $[\mathrm{al}]$

=now [already]

[ja] ja:

[yes] ye:s

waar gaat $u$ heen juffrouw

where are you going miss

Turkije denk ik

Turkey I think

10 o ma: : echt joh altijd die Hollanders naar Tur[kije]

ma: : really always those Hollanders to Tur[key]

[heh] heh heh [heh]

[heh] heh heh [heh]

ben er al heel lang niet meer geweest, (.) tien jaar denk ik? haven't been there for a very long time, (.) ten years I think?

14 Ik ben wel [zes keer] geweest

I have been [six times] already

[juffrouw u]

[teacher you]

16 S juffrouw u heeft wel geld hè, Turkije, Spanje,

teacher you do have lot of money huh, Turkey, Spain,

17 W Kreta,

Crete,

180 Turkije [weet je hoe facking $g^{\prime}$ dkoop Turkije is]

Turkey [you know how fucking cheap Turkey is]

19 B [het hele jaar ziet u Turken hier]

[all year long you see Turks here]

20 o je gaat met duizend euro [je hebt daar vierduizend]

you go with a thousand euros and [you have four thousand there]

21 B [ (Omer) (.) dan gaat u nog naar Turkije]

[(Omer) (.) and then still you go to Turkey] 


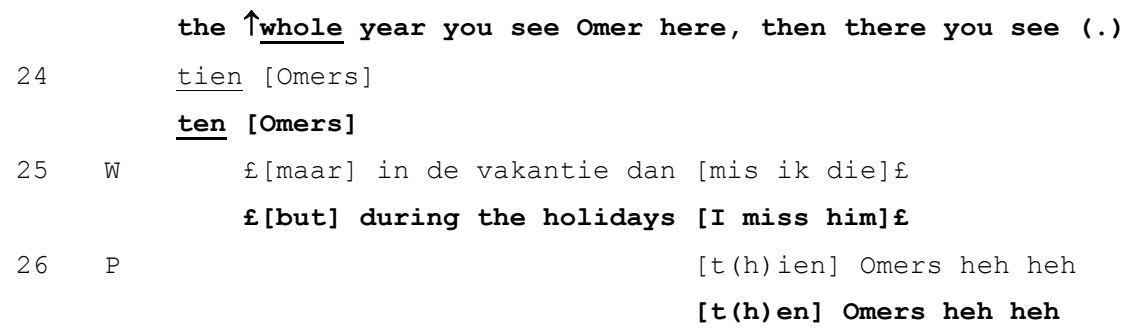

In line 1, Omer joins the conversation that I was having with Ms. Willems, followed by Stefan in line 6. Their comments on Ms. Willems' early holiday planning - a topic unusual for teacher-pupil interactions - mark an entrance into an intimate conversational sphere. Ms. Willems engages with their questions in lines 3, 7, and 9, showing acceptance of this. Omer's turn in line 10 ("ma:: really always those Hollanders to Turkey") further marks this sphere of intimacy and informality. It is also this turn that makes ethnic membership categories relevant in this interaction.

Omer's turn starts with "ma::," which can be heard as the start to a complaint. It takes Ms. Willems' proposed activity (going on holidays to Turkey) as typical for her (assigned) category Hollander. The extreme case formulation ("always to Turkey") makes it a "legitimate complainable" (Pomerantz 1986, 227), and this exaggeration makes the turn sound like mockery rather than a serious complaint. It can thus be interpreted as a case of jocular mockery by a non-member of the mocked category. Since it is furthermore directed at the teacher, Omer's comment could be risky. It might come across as improper or rude, which can be seen as another display that Omer sees this interaction as "one in which he may produce such talk; i.e. [that it] is informal/intimate" (Jefferson, Sacks, and Schegloff 1987, 160). It also illustrates the unidirectionality of restrictions against mockery by nonmembers of the mocked category: it was not treated as a heavy transgression for pupils categorized as buitenlander to mock people categorized as Nederlander.

My laughter in line 11 indicates surprise (Billig 2005) and furthermore suggests that I recognize the potential transgressive nature of this comment, but interpret it as 'mild' enough to warrant laughter. Ms. Willems disattends the potential transgression: she does not attend to the complaint in Omer's turn, but reacts to the content of Omer's comment lightheartedly in lines 1214, again signaling acceptance of the more informal, intimate sphere of conversation. Stefan then also engages in a potential transgression in raising the topic of Ms. Willems' financial situation. Again, Ms. Willems does not 
object, but instead cooperates with Stefan's turn in line 16, casually boasting about the places she has recently visited.

In line 19, Ben enters into the conversation with what later turns out to be the run-up to a joke. "The whole year you see Turks here" is an extreme case formulation similar to Omer's complaint in line 10 about Hollanders "always" going to Turkey. In that turn, Omer suggested that it is annoying that Hollanders go to Turkey; now Ben suggests that it is annoying that Ms. Willems sees Turken all year. The joke, which further develops in line 21, is disguised as a comment directed at Ms. Willems, while it can be recognized to actually target Omer through the category 'Turken.' Omer is speaking at the same time, however, and does not acknowledge reception of the joke. Ms. Willems also does not react, and I laugh in line 22. When, in line 23, Ben has the floor to himself, he delivers the joke again in a slightly adapted and more fluent version. The first part of the joke, which previously was 'the whole year you see Turks here' has now become 'the whole year you see Omer here.' It now ends with a new punchline in which Ben has made Omer into a madeup category of 'Omers': "there you see ten Omers." It suggests that 'Omer' is an interchangeable member of a category of 'Omers' that Ms. Willems would not voluntarily be surrounded with. The joke is thus now phrased as an individualized attack to his friend Omer (instead of one against the entire category 'Turks'), though disguised as a comment to Ms. Willems (who is indicated by the 'you' in the turn).

All Ben's turns are delivered without laughter particles (Jefferson 1979). Nevertheless, the delay in delivery, the repetition, the exaggerated stress of certain words (e.g. "the whole year"), and absurdity ('Omers' as a membership category) make it recognizable as a so-called 'deadpan' delivery of humor, that is, humor or irony delivered without overt markers of humorous intent (Attardo et al. 2003) and more specifically, jocular mockery (Holmes and Marra 2002). It can furthermore be interpreted as Ben's return insult to Omer's earlier mocking comment about tatta's going to Turkey on vacation. Omer, who is the butt of the joke, does not respond - not affiliatively nor disaffiliatively. Ms. Williams acknowledges Ben's joke with smile voice in line 24, but she does not exactly laugh (Jefferson 2004). Omer has thus probably also heard it (or at least has been able to) but does not react, at least verbally. Ms. Willems' reply, in which she explains that she would 'miss Omer during the holidays' can be heard as ironic (Clift 1999). In line 25, I am laughing and repeating part of Ben's punch line. Omer himself does not respond, and quickly after this interaction, the topic of conversation turned to the grades of a test.

It seems like this interaction had all the possible elements that would allow for interactionally cooperative (or 'successful') jocular mockery to occur. 


\section{Nederlanders and buitenlanders}

There was a previous atmosphere of lighthearted mockery and joking surrounding ethnic categories and category-incumbent characteristics; Ben and Omer were good friends who often engaged in mutual banter; and Ben's joke about the category Turken is carefully designed (the word Turken is even absent in the second delivery of the joke). Nevertheless, Omer, who is the butt of the joke and the only participant who was present who could ratify the joke about his own category, does not show overt offense, but also does not join in with laughter or make return insults (Billig 2005; Jefferson 1979). This shows that even in optimal circumstances, it was difficult for members of the category Nederlander to engage in successful jocular mockery about other ethnic categories.

The examples in this section have demonstrated that jocular mockery with ethnic categories in class $3 / 4 \mathrm{~b}$ was restricted by the category of the 'joker.' Pupils categorized as Marokkaan, Turk or buitenlander often jokingly mocked their own or other people's categories, including the category Nederlander. This was much more difficult, if not impossible, to do for pupils categorized as Nederlander. Example 4.6 has shown how it can go wrong, and Example 4.7 showed that even in apparently optimal interactional circumstances, such jocular mockery may not receive the same affiliative reactions as jocular mockery by members of the mocked category.

\subsection{Conclusion}

As seen in the previous and the current chapter, pupils of class $3 / 4 \mathrm{~b}$ frequently referred to the categories Marokkaan, Turk, buitenlander, and Nederlander. In Chapter 3, I explored how pupils endowed those categories with local social meanings and how they negotiated those meanings and associations. In this chapter, I have attempted to work toward a possible answer to the question of why these pupils referred to categories so often. I have argued that references to these categories enabled pupils to make interactional moves. The categories had a wide array of associations (e.g. 'members of different categories have different customs'; 'the category Marokkaan is discriminated against,' etc.), and by mentioning a category, pupils evoked those associations and in so doing could make interactional moves. If, through category mentions, interactants can achieve certain moves in interaction, then a speaker can also mobilize them strategically, when aiming to achieve such a move. For instance, if mentioning the category Marokkaan in a certain context tends to cause an interactant to step down from a dominant 'expert' position and be open to receive rather than impart knowledge, then mentioning the category Marokkaan can become a strategy, aimed at achieving that shift of power positions. 
Omer in Example 4.1, for instance, categorized himself as buitenlander and thereby evoked the idea that members of different categories have different customs, and that therefore, I (as a Nederlander) was not in the position to judge him. In Example 4.2, Amine categorized himself as Marokkaan and evoked the association of discrimination when he was being punished by a teacher, potentially in a bid to recruit his peers to defend him by positioning himself as the victim of racism.

In Section 4.4, I discussed the role of humor with and about ethnic categories in class 3/4b, which the pupils in Example 4.3 framed as the main marker of difference between the locally established categories tatta's (i.e. Nederlanders) and buitenlanders. Example 4.4 demonstrated such an instance of jocular mockery with ethnic labels. Through vari-directional double voicing, pupils with a migration background insulted the category of which they themselves, or their conversational partner, were seen to be a member. Such jocular use of labels seemed to function to mitigate the effects of potentially hurtful, stigmatizing voices in Dutch society and allowed pupils to comment on structures of inequality and marginalization.

The interactions in Section 4.5 showed how pupils' own perceived category membership restricted the interactional moves they could accomplish with ethnic labels. Jocular mockery through vari-directional double voicing was interactionally complex to achieve for pupils labelled as Nederlander: they could easily be understood to 'do,' rather than 'imitate,' stigmatizing discourse. The interaction in Example 4.5 and 4.6 illustrated those restrictions. Example 4.7 demonstrated that, even in optimal circumstances, it was difficult for a pupil labelled Nederlander to achieve jocular mockery with an ethnic label reference. The practice of referring to ethnic labels thus also marked different groups of pupils: those who could engage in interactional work with ethnic labels without effort (i.e. pupils labelled as Marokkaan, Turk or buitenlander) and those who could not (i.e. pupils labelled as Nederlander).

In sum, references to the categories Marokkaan, Turk, buitenlander, and Nederlander helped speakers to "achieve the interactional outcomes that they are designing their actions to achieve" (Whitehead 2012, 1261), or in other words: they were useful. One consequence of this practice is that it constitutes a mechanism by which ethnic or descent categories are constantly reproduced through ordinary interactions. Their usefulness provides speakers with motivation to keep using them; and the continued use reifies the existence of those distinct categories of people (Whitehead 2012). This can be recognized in the interactions analyzed in the next chapter, which analyzes categorizations in interactions involving teachers. 
134 Nederlanders and buitenlanders 


\section{Categorization in interactions involving teachers}

\section{Field notes, 8 May 2017}

The pupils are entering the Biology classroom. I go in with them, greet the teacher, and find a spot at the back of the classroom. Ms. Bos starts the class, which is about the digestion system. Seemingly out of nowhere, Meryem interrupts Ms. Bos and asks: "How come foreign grandparents are always fat, and Dutch grandparents skinny?" Ms. Bos smiles and answers: "Well, Turkish and Moroccan people visit each other very often, and when they do, there tends to be a lot of food. Dutch people, on the other hand, have dinner at five and if you're not there at that precise moment, well, that's too bad for you." The pupils laugh. "It's true, teacher! Dutch families consist of four people, and they cook exactly enough just for those four."

\subsection{Introduction}

In the previous chapters of this dissertation, I paid attention to how pupils like Meryem categorized themselves and each other using the terms Marokkaan, Turk, buitenlander and Nederlander in interaction. I analyzed how the pupils negotiated the meanings of those categories (Chapter 3) and I reviewed some functions of categories in pupils' interactions (Chapter 4). I emphasized that ethnic category terms are complex and locally contingent, and subject to negotiation and change, and that pupils used them to engage in the management of everyday social relations and to construct local social hierarchies. Pupils were not the only important actors at school, however. As the vignette at the start of this chapter illustrates, teachers at South High School were also involved in categorization processes, and they also formed part of the interactional processes by which categories were endowed with meanings and functions at South High School. In order to highlight this, this chapter focuses on teachers' engagement with the categories Marokkaan, Turk, buitenlander and Nederlander. I ask what role ethnic categorizations played in interactions involving teachers, and to what extent, in these interactions, we can see reflected some of the meaning negotiations and interactional functions as analyzed among the pupils in Chapters 3 and 4.

\footnotetext{
* Parts of this chapter have previously appeared in: van de Weerd, Pomme. 2020. "Categorization in the classroom: a comparison of teachers' and students' use of ethnic categories." Journal of Multicultural Discourses. Advance online publication. doi: 10.1080/17447143.2020.1780243
} 


\section{Nederlanders and buitenlanders}

Of course, just as pupils cannot be regarded as one homogeneous entity with regard to their interpretation of categories, so must all teachers that I describe in the chapter be considered individuals. Their behavior cannot be analyzed as symptomatic of a single entity with a unified understanding of pupils. What this chapter aims to illustrate, however, is that despite individual differences between teachers, there were certain tendencies recognizable. I will argue that while pupils shifted between localized uses of categories (as described in Chapters 3 and 4) and more conventional uses that are widespread in Dutch society (namely that they are indicative of a lack of belonging, or that they are stigmatized categories with negative associations), teachers mostly appeared to interpret self-categorizations as Turk, Marokkaan and foreigner as evoking those latter, more conventional associations. Furthermore, I will demonstrate that while some teachers problematized their pupils' selfcategorizations as 'not Dutch,' they also ethnicized pupils in other moments. Finally, the chapter illustrates that, like the pupils, teachers could also use categorizations in interaction strategically, to achieve moves in interaction.

The chapter is organized as follows. First, I shortly review previous literature on categorization by pupils and teachers and identify the gaps that I aim to address in this chapter. Then, in Section 5.3, I discuss how teachers problematized pupils' self-categorizations. Section 5.4 focuses on moments in which teachers categorized pupils, either among themselves, in interaction with me, or with the pupils. I end by summing up some of the implications of these findings.

\subsection{Teacher and pupil categorizations in previous literature}

In Chapter 3 I established, in line with previous research, that ethnic category terms are complex and locally contingent, and that they are subject to negotiation and change. Adolescents and children, often observed in the context of formal schooling, have been reported to engage in reconfigurations of ethnic (and other) categories and the terminology surrounding them. They may use an ethnic label with new meanings (Lee 2009), or they may alternate between different ethnic or racial categories in different contexts (e.g. Nørreby and Møller 2015). As Lee (2009, 40) argues, "[r]ather than using predetermined demographic categories, youth adopt their own categories to label ethnicity." Many of these descriptions of reconfigured use of ethnic labels do not include descriptions of teachers' engagement with pupils' categorizations, however.

Several other studies have reported on how teachers ethnically categorized their pupils, and often, how pupils disaligned with this. In a Dutch primary school classroom, for instance, Koole and Hanson (2002) showed 
how the teacher oriented to certain pupils as 'Moroccan,' and attached preconceived ideas about 'Moroccan' or 'Muslim' culture to this category (such as maintaining strict separation between boys and girls). The pupils themselves did not use the category label 'Moroccan' for themselves on their own initiative, and disaligned with the characteristics that the teacher associated with 'their' category. Martín Rojo (2008) also demonstrates how pupils disaligned with being ethnically categorized by their teacher. In a Spanish multicultural school, the teacher categorized her pupils as "locals' and 'immigrants,' 'Españoles' and 'Marroquies' or 'Latinoamericanos"' (Martín Rojo 2008, 33, italics in original). The pupils themselves resisted those categorizations, for instance by joking or being silent in reaction to teacher's claims about what she framed as 'their' categories. Duff (2002) and Talmy (2009) have highlighted the mechanisms by which categorizations found their way into the classroom through teacher-led activities. In these studies, teachers seemingly aimed to create inclusive and respectful classroom environments, for example by calling upon pupils of non-local origin to explain 'their' culture to their peers, resulting in effectively othering these pupils. Teachers have thus been observed to categorize their pupils, and in response, pupils challenged those categorizations.

Thus, many studies have focused either on pupils' categorizations of themselves, or on pupils' resistance to their categorization by teachers. This chapter, in contrast, focuses on how teachers of class $3 / 4 \mathrm{~b}$ related to, and engaged with, categorizations that were used primarily by pupils. In the following, I first analyze how teachers of class $3 / 4 \mathrm{~b}$ problematized their pupils' categorizations in interaction with me and with the pupils themselves. Thereafter, I describe how teachers ethnically categorized pupils, in interaction with me as well as with each other and with the pupils.

\subsection{Teachers' problematizations of categories}

I start this section with a partial repetition of an interaction that I analyzed previously as Example 3.9 in Chapter 3. This serves as an example by which to reiterate three characteristics of pupils' categorization that are key to the interpretation of, and comparison with, teachers' discourses surrounding categories in the following paragraphs. The extract illustrates, first, that pupils' discourse about the categories Marokkaan, Turk, buitenlander and Nederlander often revolved around the establishment and negotiation of local social hierarchies, in which Marokkaan, Turk and buitenlander were more prestigious than Nederlander, second, that pupils framed these categories as embedded in the Netherlands rather than in (for instance) Morocco or 


\section{Nederlanders and buitenlanders}

Turkey; and, finally, that pupils' discourses about those categories nevertheless often echoed wider societal discourses.

Example 5.1. 16 June 2017. Group conversation with Farida (F), Yildiz (Y), Meryem (M), Amira (A), Dounia (D), and the researcher (P).

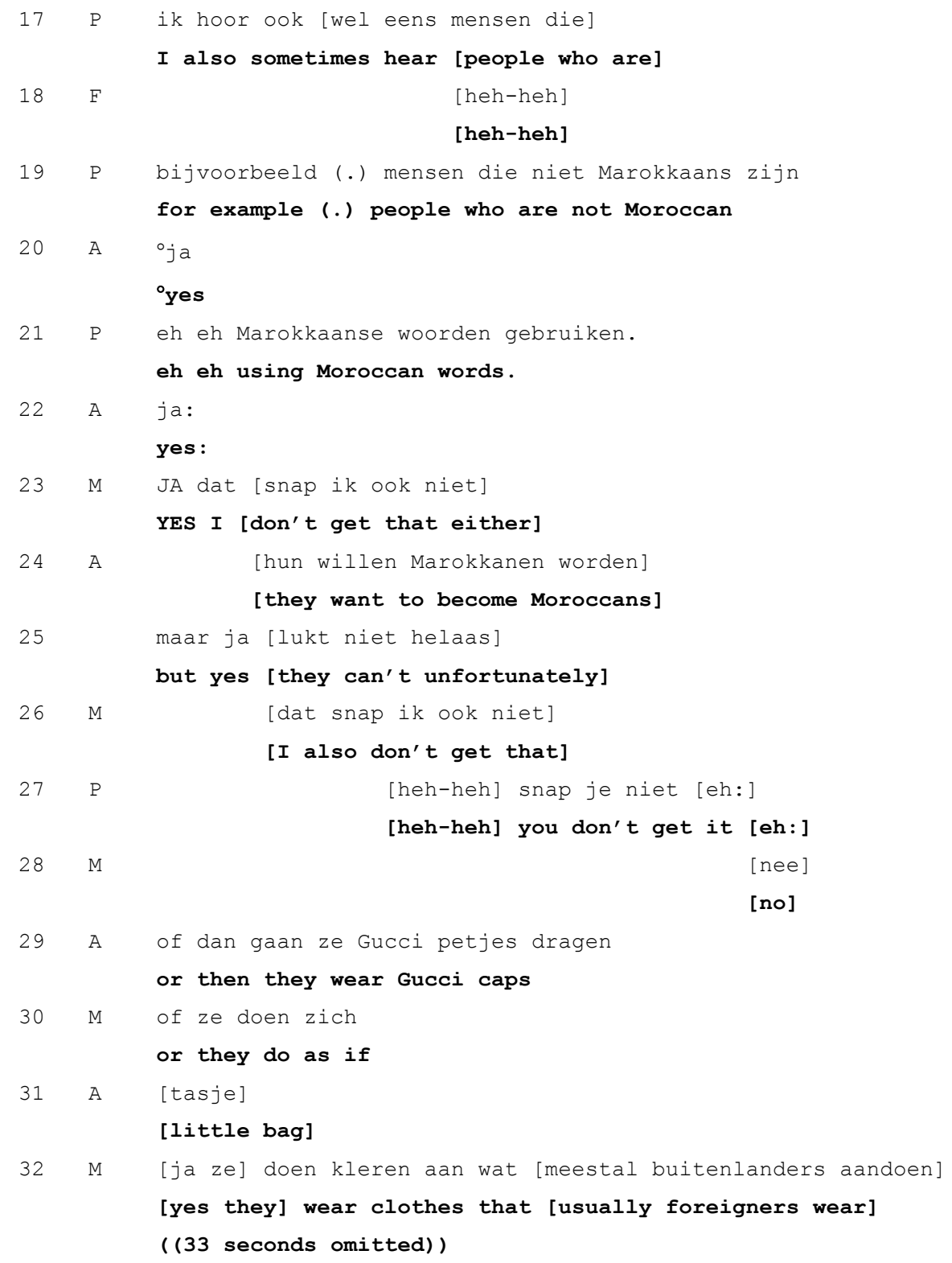




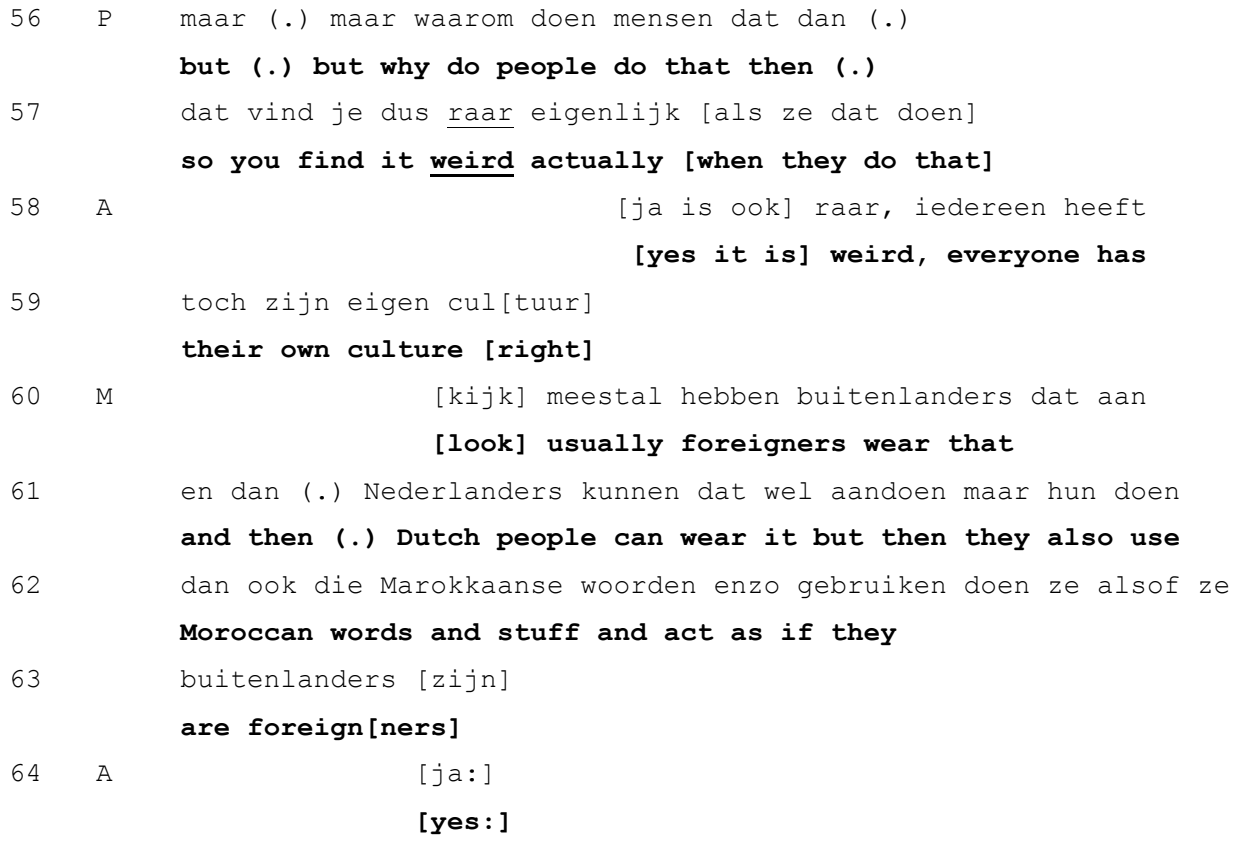

As illustrated by the interaction in Example 5.1, for these pupils, being a member of the categories 'Moroccan,' 'Turk' or 'foreigner' was prestigious, and was thus worthy of imitation (see lines 44-47), while being 'Dutch' was unprestigious. This is a reversal of the stigma that the categories 'Moroccan,' 'Turk' or 'foreigner' carry in Dutch society more generally (e.g. Bouabid 2016), and that these pupils also dealt with in their daily lives. In South High School, where many peers had similar migration backgrounds, that background constituted social capital. Many references to ethnic categories in pupil interaction alluded to those local hierarchies, and categorization had an important social function in the management of local social relations. Example 5.1 also illustrates how pupils framed the categories 'Moroccan,' 'Turk' and 'foreigner' as embedded in the Netherlands. When Amira says 'Yes, it is weird, everyone has their own culture, right' (lines 42-43), she does not talk about the culture of people in Morocco. The elements that the pupils mentioned in the lines before this transcript starts (see Example 3.9 on page 89) as being authentic for 'foreigners,' such as the use of certain vocabulary and clothing, are characteristic of a social category that pupils in this context constructed of people with Moroccan or Turkish migration backgrounds in the Netherlands. These were, one could say, localized categorical associations. Third, the extract illustrates that even though the culture that the pupils in this extract referred to as 'the culture of Moroccans' was different from the image of 
'Moroccan culture' as it is put forward in much popular or political discourse (that is, as connected to certain religious and cultural practices, see Slootman 2016), the way pupils talked about that culture had striking similarities to prominent culturalist discourse in the Netherlands (Ghorashi 2017). In line 42, where Amira posits that "everyone has their own culture,' she echoes such culturalist discourse in portraying culture as something that one "carries with them' - as "static, a-historical and essentialist ... with fixed boundaries" (Anthias 2013, 324). Pupils thus did not always or only use categories in localized ways that were radically different from wider societal discourse. The more 'localized' meanings co-existed with more 'conventional' meanings of categories in pupils' discourse.

In sum, pupils had endowed the categories Marokkaan, Turk, buitenlander, and Nederlander with local meanings: they had local social functions in negotiating social positions at school and were used to describe social positions that were embedded in the Netherlands (and thus did not necessarily indicate a lack of belonging to the Netherlands or feelings of belonging to another country). At the same time, their discourse about categories showed striking similarities to broader societal discourses surrounding those categories, for instance in portraying 'their' culture as different from 'Dutch' culture. In some contexts, pupils' use of categories evoked more locally-specific meanings, and at other times, they evoked the more conventional meanings of categories that echoed wider societal discourse.

With their shifts between localized and conventional uses of categories, and their playful manipulation of those distinctions, pupils demonstrated awareness that a Marokkaan, Turk, or a 'foreigner' could have different connotations to them than they do to many other people, for whom these labels may be charged with negative associations. The following example illustrates how a pupil used the label buitenlander to conjure up some of those more conventional associations in interaction with a teacher. The interaction occurred during a Care and Well-being class with Ms. Smit. Ms. Smit was, generally, stricter and more punitive than most other teachers I observed, and pupils often expressed to me that they did not like her. During the interaction, Hatice, Amira, and Dounia were seated at a table, supposedly working on an assignment but mostly chatting together. I was sitting with them, not participating much in their conversation, but mostly listening in.

Example 5.2. 19 December 2017. Field notes, Care and Well-being class. I'm sitting with Hatice, Amira and Dounia who are talking and laughing. Ms. Smit comes by and scolds them, telling them to concentrate on their work. This exchange follows: 
Hatice: "Always those foreigners, isn't it..." ("Altijd die buitenlanders, hè"). Ms. Smit, rather forceful: "You're not foreigners, you're Dutch!" (Jullie zijn geen buitenlanders, jullie zijn Nederlanders").

This example illustrates that pupils did not only draw on their own, localized meaning constructions of labels such as buitenlander ('foreigner'), but that they could also evoke other discourses surrounding this category. In this case, Hatice's use of the category 'foreigner' does not evoke the prestigious and positive localized associations that I described in reference to Example 5.1 and in Chapters 3 and 4. Rather, she has taken on the voice of a racist Other in an instance of vari-directional double voicing (Bakhtin 1981), echoing a societal discourse that stigmatizes people with migration background, and in which buitenlander has negative connotations: 'It is always those foreigners that misbehave.' She can be seen to engage in 'say foring'(Goffman 1981, 150; Jaspers 2005a), that is, she 'speaks for' Ms. Smit, offering a categorical explanation for her and her friends' behavior (since she, Amira and Dounia categorized themselves as 'foreigner'). She thus builds on what could be regarded a 'conventional' use of this category in order to frame Ms. Smit's supposed thoughts as potentially racist. She does this in a somewhat playful way, eliciting laughter from her friends.

Ms. Smit sounds corrective and irritated in her reaction to this categorization. This can be understood in multiple ways. Potentially, Ms. Smit orients to Hatice's comment as a challenge to her authority (similar to how, in Example 4.2 on page 109, Amine challenged Ms. Bos by accusing her of singling him out 'because he is Moroccan'). Her correction of Hatice's selfcategorization can then be understood as a pedagogical move and a reinforcement of that authority. That is, her comment 'You're not foreigners, you're Dutch!' might be a way for her to say that Hatice's categorization is irrelevant (and that they must just do their schoolwork). Another possibility is that Ms. Smit reacts to the Hatice's categorization, as she corrects it by saying that the pupils are 'Dutch.' This suggests that she follows Hatice's 'conventional' usage of the category foreigner - one that carries associations of othering and stigmatization - and therefore denies that she would categorize her pupils that way. In other words, Ms. Smit may have understood the comment to be a disguised accusation of racism, and her correction might have been her way of denying racism and ending the conversation. In sum, it is not entirely clear from this example whether Ms. Smit is primarily involved in a struggle for authority with a pupil that challenges her, or that she disagrees with the categorization itself. In any case, Ms. Smit expresses in the interaction that, for these pupils, a self-categorization as 'Dutch' is preferable over one as 'foreigner.' 


\section{Nederlanders and buitenlanders}

The teacher in the next example, Ms. Jansen, was 3/4b's head teacher at the time. In the example, which comes from a short informal interview I had with her, Ms. Jansen also (and more unequivocally) expressed that she would like her pupils to self-categorize as 'Dutch.' The transcript starts just after I expressed my surprise at pupils' categorizations to her.

Example 5.3. 16 June 2017. Conversation between Ms. Jansen (J) and the researcher (Pomme, $\mathrm{P}$ in the transcript).

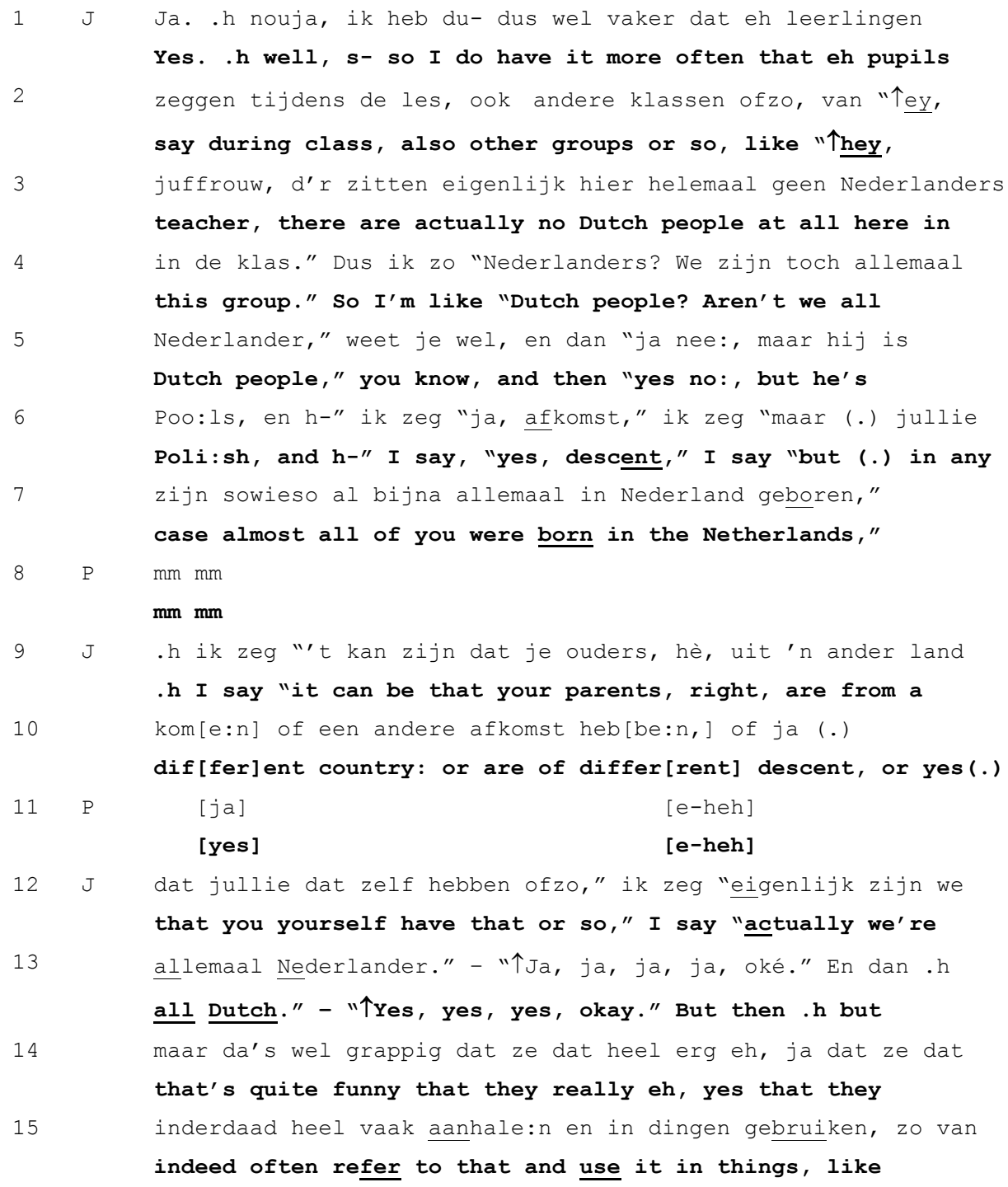




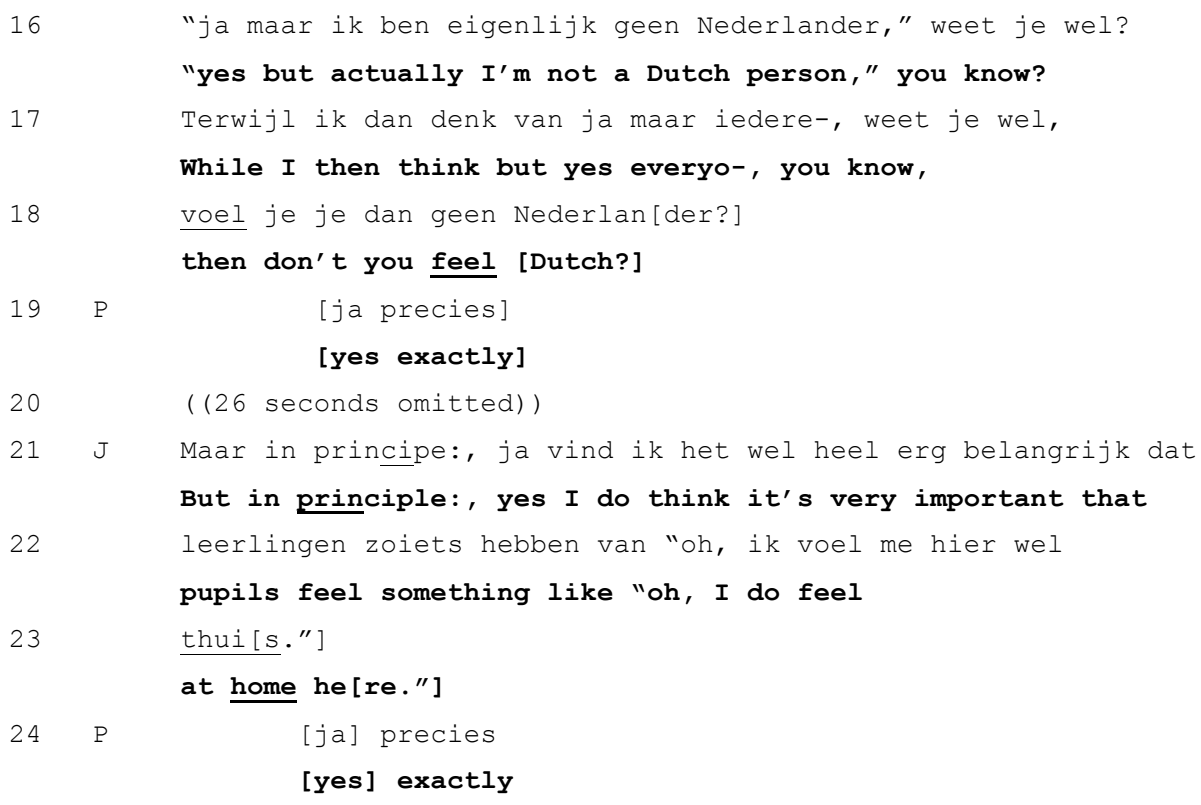

16

17

18

$19 \mathrm{P}$

( (26 seconds omitted))

$21 \mathrm{~J}$ Maar in principe:, ja vind ik het wel heel erg belangrijk dat

But in principle:, yes I do think it's very important that

22

23

$24 P$

"ja mar ik ben eigenlijk geen Nederlander," weet je wel?

"yes but actually I'm not a Dutch person," you know?

Terwijl ik dan denk van ja maar iedere-, weet je wel,

While I then think but yes everyo-, you know,

voel je je dan geen Nederlan[der?]

then don't you feel [Dutch?]

[ja precies]

[yes exactly]

leerlingen zoiets hebben van "oh, ik voel me hier wel

pupils feel something like "oh, I do feel

thui $[s \cdot "]$

at home he [re."]

[ja] precies

[yes] exactly

In this extract, Ms. Jansen recounts an example of a (largely hypothetical) conversation between herself and a pupil about category terms. By saying, 'Aren't we all Dutch' (lines 4-5), Ms. Jansen includes her pupils in the category of 'Dutch' with the pronoun 'we' (cf. Lerner and Kitzinger 2007). The (hypothetical) pupil, however, rejects this by categorizing a third person as 'Polish' (line 6). The selection of this category is interesting, as I rarely heard this label used by teachers or pupils during my time at South High School. At least among the pupils of class $3 / 4 \mathrm{~b}$, it did not seem to be a tokenized membership category in the same way 'Moroccan' or 'Turk' were, Possibly, Ms. Jansen chose this as an example to avoid talking about the more charged categories 'Turk' and 'Moroccan,' i.e., to avoid being heard (by me) to engage in, or referring to, potentially racist or stereotyping discourse by merely mentioning the label (Pollock 2004).

In lines 6-13, Ms. Jansen constructs the category 'Dutch' as based on something other than descent, but instead, possibly, about a 'feeling' (line 1718). This may serve to display to her pupils (or to me, in the interview) that she disaligns with the widespread use of the term 'Dutch' on the basis of descent, and thus exclusively referring to people without migration backgrounds (Ghorashi 2006; Eijberts and Ghorashi 2017). She appears to say that regardless of potential stigmatization or discrimination, she wants her pupils to feel they are 'Dutch' anyway. This implies that, to her, being able or willing to self-categorize as 'Dutch' is preferable over the use of other labels. 


\section{Nederlanders and buitenlanders}

Ms. Jansen ends with the comment 'But in principle I think it's so important that pupils feel at home here' (lines 27-28). It is not entirely clear what she refers to with the word 'here.' It could be 'here in the Netherlands' (since we have been talking about the category Nederlander), or 'here' at school (as this is the context from which she has been speaking). Anyway, it demonstrates her perception of a direct link between pupils' usage of labels other than 'Dutch' and potentially not 'feeling at home.' This sentiment may have come forth from a wider societal discourse that problematizes migrants' and their descendants' feelings of 'belonging' to the nation, and that interprets the use of labels such as buitenlander, Marokkaan and Turk as expressions of (a lack of) those feelings.

In sum, Example 5.2 and 5.3 illustrate a few characteristics of the dynamics of categorization in teachers' discourse. First, they showed that some teachers, in interaction with pupils and with me, problematized their pupils' self-categorizations as 'foreigners,' 'Moroccans,' or 'Turks.' In Example 5.3, the problematization seemed to come forth from a concern with pupils' feelings of being 'at home' or 'belonging,' showing an interpretation of categories similar to conventional societal discourses that read selfcategorizations as Marokkaan, buitenlander or Turk as indicative of a lack of belonging. Example 5.2 illustrated, first, that pupils did not only evoke localized associations when using categories (such as in Example 5.1 and the examples in Chapters 3 and 4), but that they also used them with their more conventional associations. In that example, Hatice played with the existence of such conventional discourses surrounding categories by framing a teacher as potentially racist by portraying her as categorizing them in the context of a negative appraisal. The teacher corrects the categorization as 'foreigners' into one as 'Dutch.' Both examples suggest that while pupils shifted between localized and conventional uses of categories, teachers mainly displayed orientations to categories in reference to conventional associations, which sometimes found expression as worry about self-categorizations (Example 5.2), and other times as corrections of self-categorizations (Example 5.3).

\subsection{Teachers categorizing pupils}

The examples in the previous section showed how some teachers, such as Ms. Smit and Ms. Jansen, problematized their pupils' self-categorizations as 'foreigners,' 'Moroccans,' or 'Turks' in interaction. The examples in the current section show that, at the same time, many teachers categorized their pupils as 'not Dutch.' Although teachers did not often initiate categorizations of their pupils in direct interaction with them, they regularly displayed understandings of their pupils with migration background as different from those without 
such a background. Most frequently, this happened in interactions between teachers. For instance, once during a break in the teachers' lounge, a teacher told me that she found it difficult to teach groups with many 'allochthones. ${ }^{25}$ When I asked her why, she responded: "Well, the Islamic culture just deals with women very differently. Their mothers are holy, but other women are the opposite" (Field notes, 24 January 2017). In Example 5.4, Ms. Smit - the same teacher who in Example 5.2 corrected her pupils' self-categorization as 'foreigner' into a categorization as 'Dutch' - orients a similar ethnicizing interpretation of pupils' behavior to me.

Example 5.4. 21 March 2017. Field notes, Care and Well-being class. After cooking class, the pupils are doing dishes. I am sitting at a table nearby, writing notes, when Ms. Smit sits down next to me. Near us, Dounia is filling the sink. I think she's waiting for the water to get hot (as per Ms. Smit's instructions of a few weeks ago) because she has had the tap on for a while now. Ms. Smit scolds her: 'Turn off the tap!' Then, in a softer voice, she tells me: YYou know, water hardly costs anything in Arabic countries, and that's what you see reflected here.'

Here, Ms. Smit seems to want to convey supposed knowledge of her pupils and their customs to me, as a researcher in this class. She culturalizes Dounia by explaining her behavior as emanating from her migration background in an 'Arabic country' (i.e. Morocco), demonstrating an understanding of Dounia's migration background as relevant and consequential in this moment. The interaction also shows Ms. Smit's categorization of me as not 'Arabic' and thus benefitted by an explanation of how things work in 'Arabic countries.' Her lowered tone of voice implies that she did not want her pupils to hear her comment.

Although teachers' categorization of pupils most often occurred when they interacted with other teachers (or with me, being recruited as an accomplice in the previous example), I also witnessed a few moments where teachers categorized pupils in interaction with the pupils themselves. In the following two examples, Ms. Smit (Example 5.5) and Mr. Maassen (Example 5.6) build on categories of religion to group pupils with and without a migration background into different categories. Example 5.5 occurred during

\footnotetext{
${ }^{25}$ The term 'allochthone' was originally used to refer to all people with a migration background in the Netherlands. It has become increasingly used to refer to people with 'non-Western' migration backgrounds, in particular those who are positioned as problematic (Roggeband and van der Haar 2018). The word is not used anymore in media, political discourse, and research, but is still sometimes used outside those more formal contexts. See Chapter 1.
} 


\section{Nederlanders and buitenlanders}

a Care and Well-being class with Ms. Smit. The pupils came into the classroom in the morning, to find Ms. Smit sending them to a particular area of the classroom, the 'restaurant,' to take a vocabulary test. I was standing near the entrance of the classroom together with Ms. Smit.

Example 5.5. 18 April 2017. Care and Well-being. Participants: Ms. Smit (S), Meryem (M), Cindy (C), Jennifer (J).

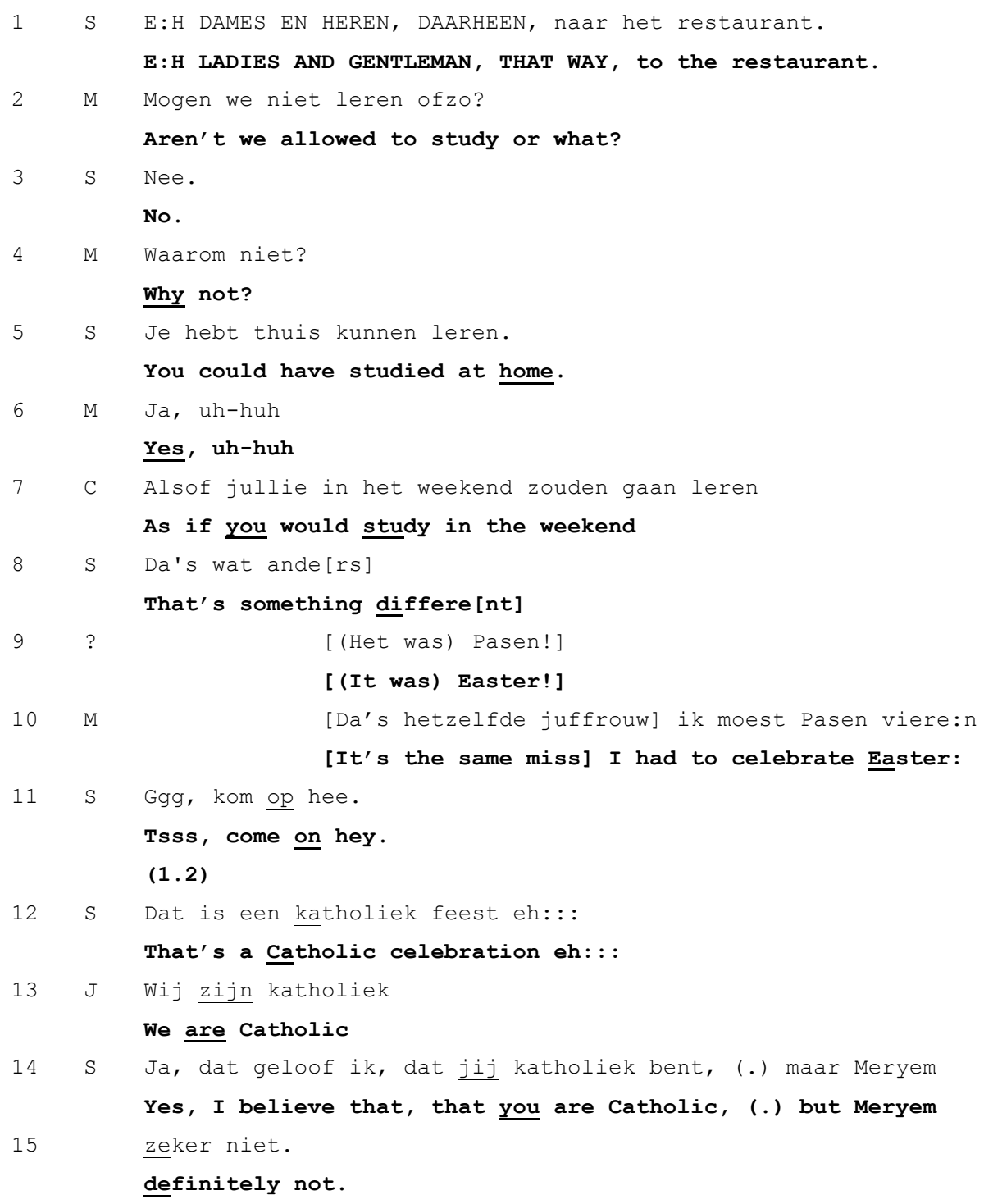


The pupils in Example 5.5 are complaining because, unlike usually, they were not allowed to go over their study materials before taking this test. At the start of the interaction, Meryem and the others engage in locally typical behavior for the category 'pupils' by standing up against the teacher. In their bid for extra study-time, they come up with reasons why they were not able to study: it was weekend (line 7), and furthermore, it was Easter (line 9). From line 11 onwards, the discussion about extra study-time becomes intertwined with a discussion about which pupil can lay claim to which kind of excuse. Meryem, in line 10, repeats the reason offered by the (unidentified) pupil in line 9 , saying that she "had to celebrate Easter." Ms. Smit's turn in line 12 ("That's a Catholic celebration") is designed as a disqualification of Meryem's excuse by categorizing Easter as a Catholic celebration, implying that Meryem is not Catholic, and that only Catholics would celebrate Easter. In other words, according to Ms. Smit, only Catholic pupils can legitimately claim Easter as an excuse for not having been able to study.

Jennifer opposes Ms. Smit in line 13, with an emphasis on zijn 'are' that shows her understanding of Ms. Smit's comment as suggesting they are not Catholic. The referent of 'we' in Jennifer's turn is ambiguous: it may refer to herself and, for instance, her family, but in the light of the preceding interaction it seems more likely that it refers to herself and the other pupils (including Meryem), thus subsuming herself and Meryem under one category. Ms. Smit's reaction shows that she interprets Jennifer's turn of as such, since she rejects it by making a distinction between both pupils. In lines 14 , with an emphasis on 'you' ("I believe that you are Catholic"), she states she believes Jennifer is Catholic, suggesting a differentiation between 'you' (Jennifer) and someone else (Meryem). In line 15, Ms. Smit makes this more explicit by stating 'Meryem definitely not' (line 15).

This example may be understood in a sense similar to that of Example 5.2 , in that in both, Ms. Smit's authority is challenged by a pupil, and in that struggle, the matter of pupils' categories enter into the interaction. In Example 5.2, Hatice's self-categorized as 'foreigner,' and Ms. Smit reacted to it by saying she was 'Dutch'; thus, Ms. Smit rejected Hatice's disguised racism accusation by rejecting Hatice's self-categorization. In Example 5.5, though, it is unclear whether Meryem actually intended to self-categorize as 'Catholic' by saying that she had to celebrate Easter (as Easter is celebrated by many people who do not self-categorize as 'Catholic' in the Netherlands) and thus whether Ms. Smit's reacts to what she has interpreted as such a selfcategorization by Meryem, or alternatively, whether Ms. Smit is the one who initiates a categorical distinction between these pupils (in line 12) where the pupils themselves did not make that same distinction in this particular interaction. Even if the motivation behind Ms. Smit's categorization is 
pedagogical - in the sense that it may have been designed to stop the pupils complaining and challenging her, and to urge them to just take the test - this example illustrates that while in one moment this led this teacher to problematize pupils' self-categorizations as 'foreigner,' 'Turk,' or 'Moroccan,' in another moment it led her to categorize pupils with a migration background as different from others without such a background. Her use of 'believe' in line 14 furthermore suggests that she did not know her pupils' religious background for a fact.

The following fieldnote recounts another instance in which a teacher displayed an orientation to his pupils as categorically different. This time, however, the categorization is part of plenary class instruction (as opposed to the more informal interaction in Example 5.5). The interaction occurred during a Societal Studies class with Mr. Maassen, a middle-aged man, who the students referred to as 'strange' (Field notes, 16 June 2017). The pupils had only two hours of Societal Studies class per week, and as a result, $\mathrm{Mr}$. Maassen did not know the pupils of class 3/4b very well (in fact, to the pupils' frustration, he regularly mispronounced their names). Nevertheless, as I observed it, the atmosphere in Mr. Maassen's class was usually alright. The class was not considered difficult, and Mr. Maassen was not very strict with the pupils. A large part of his classes took the format of plenary lectures and discussions. This was also the case during the class described in the fieldnote in Example 5.6. The topic of this class was 'the power of advertising,' and the pupils had completed an assignment from their book, which they were revising in plenary discussion. On the face of it, Mr. Maassen aimed to make this class into a learning moment not only about the power of advertising, but also about cultural or religious practices.

\title{
Example 5.6. 10 February 2017. Field notes, Societal Studies class.
}

Mr. Maassen is discussing an assignment from the textbook with the class. The question that they are discussing now asks them to state the feelings that commercials for certain products intend to evoke. One example is about rookworst (smoked pork sausage). The following interaction ensues:

\author{
Mr. Maassen: 'Meryem, do you ever eat rookworst?' \\ Meryem: $\quad$ 'No, we're not allowed.' \\ Mr. Maassen: 'Justin, why doesn't Meryem eat this?' \\ Justin: 'Haram.' \\ Mr. Maassen: 'That's right.'
}

Later, Mr. Maassen says that the feeling evoked by rookworst (i.e. the answer to the assignment question) is 'gezelligheid' (meaning something like 'conviviality'), but- 
as he says - Meryem doesn't know this, "because she hasn't experienced eating this particular sausage." He then asks the class who does eat rookworst, and some pupils, including Ali, raise their hands.

\author{
Mr. Maassen: 'Ali, do you eat rookworst?' \\ Ali: $\quad$ Yes, halal rookworst.' \\ Mr. Maassen: 'So where do you buy that?' \\ Ali: $\quad$ 'At the Turkish shop.' \\ Mr. Maassen: 'Oh, I didn't know that, I didn't know that existed.'
}

A short discussion follows between several pupils and Mr. Maassen; about whether the sausages that Ali refers to are the 'real' rookworst that Mr. Maassen was talking about, or another kind of sausage.

It seems that in this example, Mr. Maassen's aim by selecting different pupils to speak, is to acknowledge, show acceptance of, and create dialogue about differences between pupils in his classroom. His selection of speakers, and reactions to their contributions, suggests an orientation to pupils with migration backgrounds as different from those without migration background, in this case in terms of their food consumption. His selection of Meryem as first speaker does not seem coincidental, since he does not display any signs of her answer being surprising or dispreferred (Pomerantz and Heritage 2013). His immediate reaction to Meryem's rather cryptical answer that 'we are not allowed' is not, for instance, to ask who the 'we' is that she is referring to, or to ask why they are not allowed to eat rookworst. Instead, Mr. Maassen addresses the next question to Justin - who had no (known) migration background and was therefore categorized as Nederlander among the pupils - and asks him to explain Meryem's answer. Justin's succinct answer that rookworst is 'haram' (i.e. not allowed in Islam) is a categorical explanation, namely one that builds on religion. Rather than checking Justin's answer with Meryem, Mr. Maassen ratifies it as correct himself.

All these interactional details suggest that the teacher envisioned this interaction and intended it to spark a conversation about the cultural and religious aspects of certain foods. After stating the purported answer to the assignment's question - namely that a commercial for rookworst supposedly evokes a feeling of gezelligheid 'conviviality' - he 'excuses' Meryem for not knowing. The teacher then turns to the class and asks who does eat rookworst. Many pupils raise their hands, and the teacher selects Ali - who had a Moroccan migration background - to explain himself. Mr. Maassen's question to Ali sounds different from when he called upon Meryem before. It sounds genuinely interested, as well as surprised, rather than aimed at using an 
expected answer as a teaching moment. Indeed, Ali's answer that he buys 'halal rookworst at the Turkish shop surprises the teacher and a short discussion ensues about the existence of halal rookworst.

Example 5.5 and Example 5.6 show that not only pupils engaged in categorizations of themselves as 'not Dutch,' and that teachers did not just reject those self-categorizations, but that teachers also sometimes categorized them as different from pupils without a migration background. In Example 5.5, Ms. Smit challenged a pupil with a Turkish migration background in her claim that she celebrated Easter by categorizing her as not Catholic (as opposed to another pupil who she did 'believe' to be Catholic); and in Example 5.6, Mr. Maassen displayed an expectation for certain pupils to behave according to the rules of Islam, which is often portrayed in stark opposition to 'Dutch culture and identity' (Ghorashi 2017; Jennissen et al. 2018).

The next example, Example 5.7, is similar to Example 5.6 in that it shows how some teachers categorized pupils as a result of their migration background. Example 5.7 is also unusual, as it contains more explicit othering than what I observed in most teacher-pupil interactions, and the teacher in the interaction was not one of class $3 / 4 b$ 's regular teachers, but a guest teacher. The interaction occurred during a presentation by Ms. $\mathrm{O}$, a guest speaker from an organ donation organization. Ms. O visited different schools to discuss organ donation, emphasizing the importance of registering one's preference to be a donor or a non-donor. Her presentation was scheduled to last about an hour, during a Care and Well-being class. It did not relate to any of the topics discussed previously in Care and Well-being classes but appeared to be part of an ongoing national campaign to increase awareness of the possibility of organ donation. ${ }^{26}$ The pupils were seated in a half circle around the guest speaker, and the teachers and I were nearby, listening as well. Ms. O began her presentation with a personal story about the death of a close relative who donated his organs. In quite some detail, she discussed why her relative had decided to be an organ donor, and recounted the exact steps surrounding this person's death and organ donation. After this story, she started speaking about organ donation and the importance of registering one's preference to be a donor or not, as well as about the practical realities of organ donation. This part of the presentation was open to questions from, and discussion with, the

\footnotetext{
${ }^{26}$ In the Netherlands, when you turn eighteen, you receive a letter from the State with the request to register as donor or non-donor, or to register as leaving the choice to surviving relatives. In the absence of any registration, the choice is automatically left to the relatives. As of 1 July 2020, in the absence of registration the individual will automatically be registered as a donor.
} 
pupils. The transcript in Example 5.7 begins when Ms. O discusses the steps surrounding a donor's death and funeral.

Example 5.7. 6 June 2017. Care and Well-being class. The pupils are sitting around Ms. $\mathrm{O}(\mathrm{O}$ in the transcript). Yildiz $(\mathrm{Y})$, Hatice $(\mathrm{H})$ and Amira $(\mathrm{A})$ participate in the interaction.

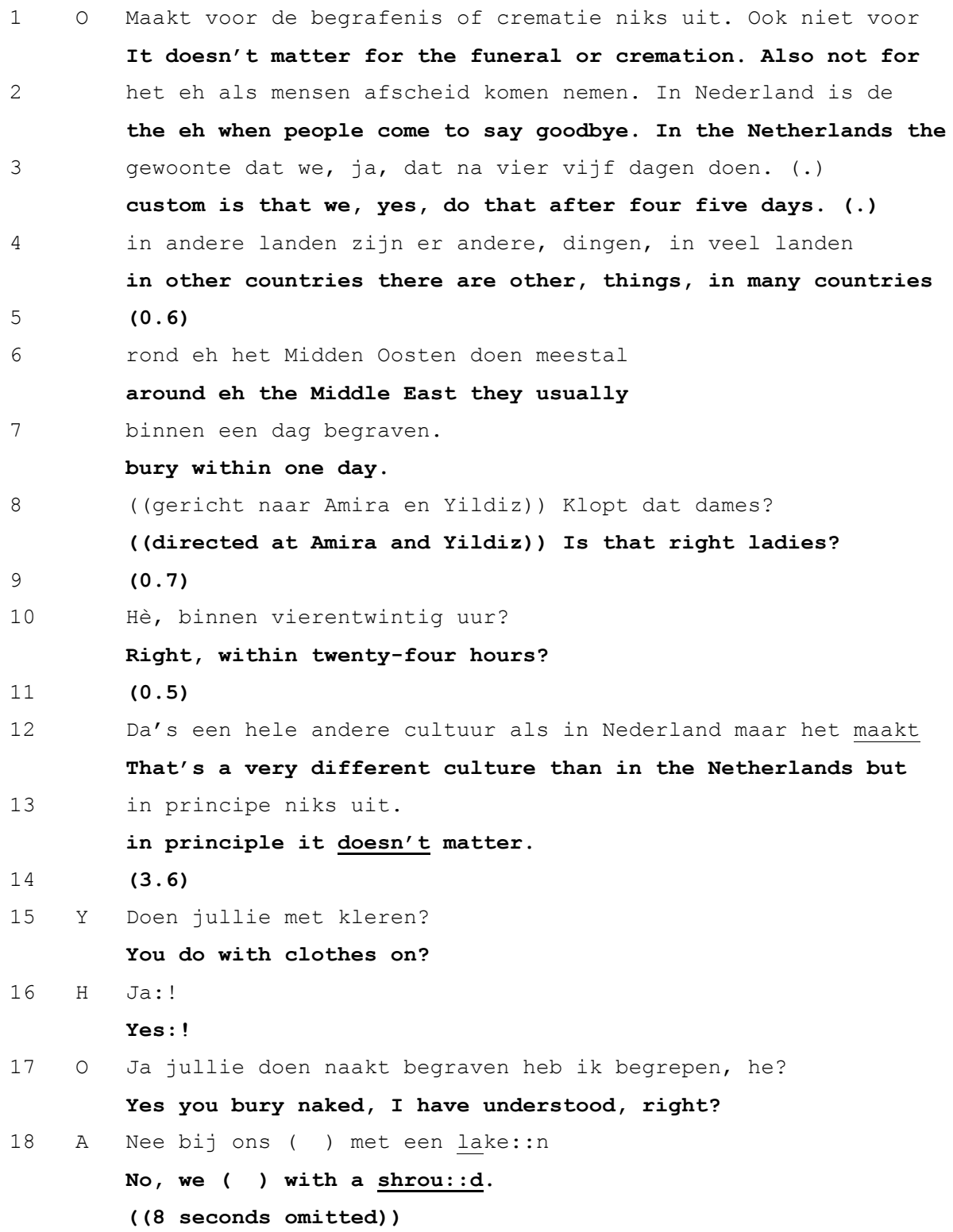




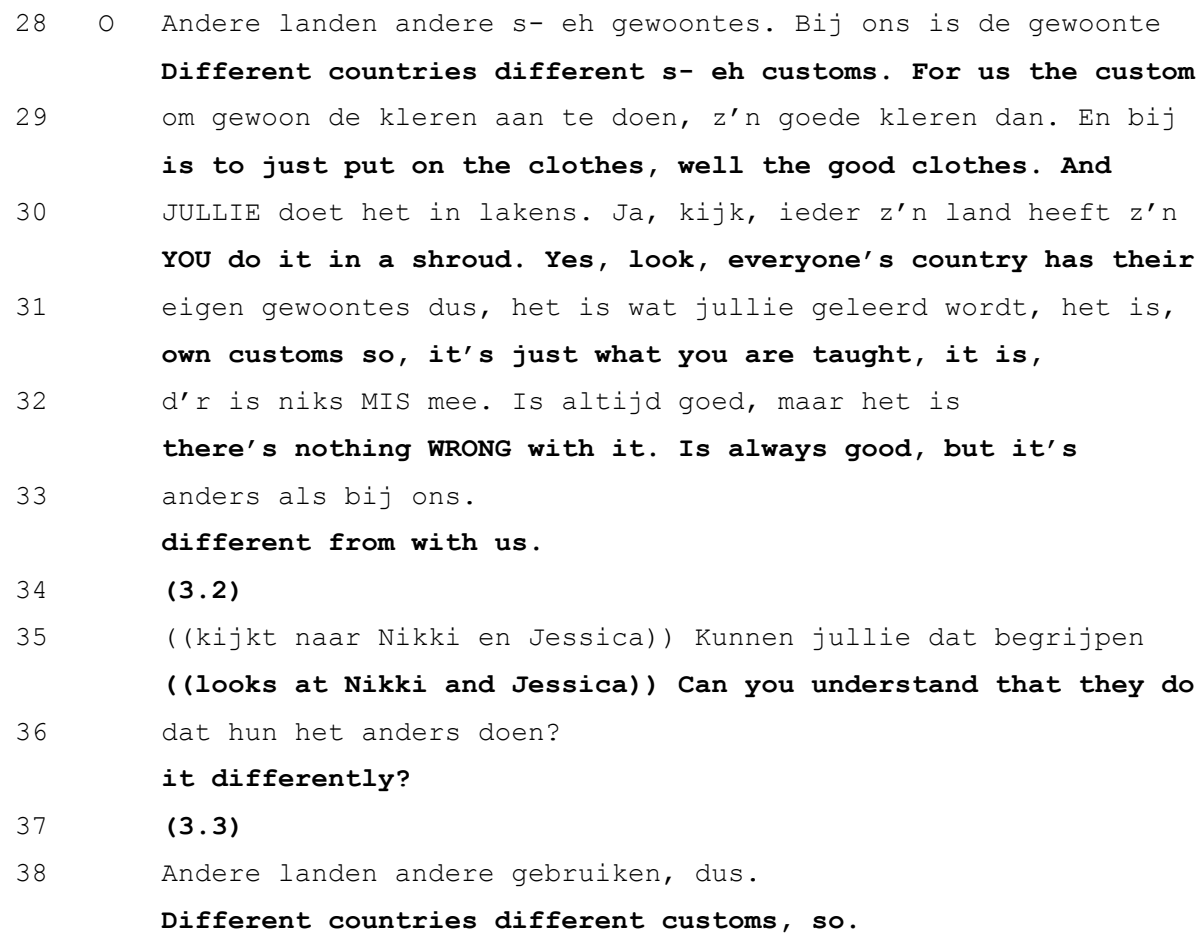

Similar to Mr. Maassen in the previous example, Ms. O's aim throughout her presentation appears to be to make the topic appeal to all pupils in the classroom - more specifically, those with and without migration backgrounds. At the same time, she produces two different groups through personal pronouns: 'we' as people in the Netherlands (lines 2-3), who are portrayed as having certain customs. In line 4-7, this becomes part of a comparison with people 'in other countries,' more specifically, the 'Middle East.' In line 8, she asks Yildiz and Amira for confirmation of her statement about the Middle East: she specifically addressed these two girls by means of body position and gaze, seemingly assuming that these girls had knowledge about funerary customs in the Middle East. Recall that Ms. O was a guest teacher and did not know any of the pupils. Directing this question at these pupils seemed to be informed by their physical appearance: Amira had black curls and dark eyes, and Yildiz wore a hijab. Both girls were born and raised in the Netherlands, though, and as was noted previously, their self-categorization as Turk and Marokkaan was rooted in the Dutch context. Neither provide the second pairpart to Ms. O's question, and Ms. O pursues it in line 10. The repeated absence of second pair-parts is salient, as Amira and Yildiz have been explicitly selected as next speakers (Hester and Francis 2004). This suggests that they 
are unwilling or uncertain about how to respond to a question about customs in the Middle East.

Ms. O then states that those (unconfirmed) funerary customs belong to a 'very different culture than in the Netherlands,' showing orientation to the notion of culture rooted in specific places (Malkki 1992). After another long silence in line 14, Yildiz asks about something Ms. O mentioned earlier, regarding whether people are buried in clothing or not. She uses the same binary categorization through pronouns as Ms. O. did earlier ('You bury in clothes?'). It seems that only from line 18, when Amira also follows the 'we/you' opposition introduced by the teacher, the other pupils with migration backgrounds present appear to understand that they are the 'jullie' ('you' plural) to whom Ms. O has been referring. A discussion between some of the pupils who referred to themselves as 'Turks' and 'Moroccans' about their customs erupts in lines 19-27. These lines have been omitted: several pupils were talking at the same time and the content of the conversation was unintelligible. After calling order, Ms. O. takes the floor again in line 28 and, by her repetition of 'different countries different customs,' reinforces an idea of culture as rooted in places, and implies that pupils with migration backgrounds not only have different customs, but that they are furthermore rooted in different places (cf. Malkki 1992). Discourses such as these reflect "widely held commonsense assumptions linking people to place, nation to territory" (Malkki 1992, 27). Throughout the interaction, none of the pupils ever characterizes their 'we' as 'Middle Eastern,' however, even if they participated in talking about 'we' and 'you' as opposing categories.

In lines 35-36, Ms. O directs herself at Nikki and Jessica, who both have light features and blond hair. She positions these girls as the 'we' that represent the supposed norm in the Netherlands. Her question whether they understand that 'they do it differently,' referring to those pupils that have been discursively set apart during the entire scene, is again followed by a long silence (line 37), in which someone chuckles softly, suggesting some discomfort. Nikki and Jessica do not answer. Finally, Ms. O repeats her statement that different countries have different customs and continues her presentation.

In this interaction, the pupils addressed as jullie ('you' plural) are endowed with a different culture (line 12) and different customs (line 28) from the people included under 'we in the Netherlands.' This reflects and reproduces a dominant idea in Dutch society of essential differences between people with, and without, migration backgrounds. It portrays pupils as representatives of their parents' birth country, leaving no space for their individual experiences that may refute what teachers think they know (cf. Duff 2002; Koole and Hanson 2002; Martín Rojo 2008). While none of the pupils overtly objected to being positioned as having a different culture, there were 
some interactional problems. The pupils did not immediately react when Ms. $\mathrm{O}$ asked them about different countries (line 8) or prompted them to assess supposedly other customs (lines 35-36). Pupils also often spoke about 'we' versus 'they,' and their 'we' was also often premised on the idea of having a 'different culture' or 'being different,' but pupils generally did not place that 'culture' (or themselves) as rooted in a different country. Aside from these few interactional issues, however, nothing pointed to any of the teachers or pupils experiencing this presentation as particularly problematic, and no one commented on it after the lesson was over.

This example, and the other examples presented in this section, illustrate how some teachers categorized their pupils with a migration background as different - culturally, religiously, or both - from their pupils without migration background, both in interaction with each other and in interaction with pupils. At the same time, as illustrated in Section 5.3, some of them problematized pupils' self-categorization as 'Moroccan,' 'Turk,' or 'foreigner.' Teachers could thus be observed to struggle with the apparent contradiction that pupils with migration background were, according to certain definitions, categorizable as 'Dutch,' but that they were also different - and positioned themselves as different - from what is conventionally understood as 'Dutch' (see also van Reekum 2012). It is important to note, however, that not all teachers always problematized their pupils' self-categorizations, or ethnicized pupils or portrayed them as 'Other.' The final example of this chapter is one of the few moments that I observed in which a teacher made explicit reference to an ethnic category in a way that closely resembled the ways in which pupils themselves used categories with localized associations in interaction. Example 5.8 occurred during a mathematics class where I overheard Mr. de Jong in conversation with Meryem and Amira. Mr. de Jong was a relatively young teacher, in his thirties, who showed much interest in my research when I first came to South High School. In the beginning phases of my research, he told me, "If you want to understand the words the pupils use, you should listen to some of the artists they listen to. I always ask them to show me their music. Pietju Bell, for instance, you should look him up on YouTube." The interaction in Example 5.8 occurred while the pupils were working on individual assignments while the teacher made rounds to see who needed help. Sometimes, like in the example below, Mr. de Jong sat down with pupils and engaged in informal chats with them.

Example 5.8. 22 May 2017. Field notes, Math class. I overhear Amira and Meryem asking Mr. de Jong, the math teacher, whether he's married - he isn't. Then Amira asks him about marrying 'just on paper.' 'You mean only for the law?" asks the teacher. Meryem: "Yeah, that's free of charge, isn't it?" 
The teacher laughs and says he's not sure. Then he jokes that Meryem "bas become a tatta" because "she wants things for free."

Mr. de Jong seemed to be more attuned than most teachers to the ways in which pupils used categories. In the fieldnote in Example 5.8, this interest in, and attunement to pupils' interactional practices, shows from his use of the word tatta, a word that originally stems from Sranan Tongo, but which is now commonly associated with youth language and is used to refer to white Dutch people without migration backgrounds. Pupils of class 3/4b often used it the way he does here, in jocular mocking contexts. Furthermore, this category's relation to being stingy was also one that pupils often invoked. The teacher's categorization of Meryem as tatta functions as a joke as it is an intentional misidentification' (Sacks 1995, I, II:417), because Meryem usually referred to herself as a 'Turk.' Pupils regularly made similar jokes in which they intentionally misidentified each other in order to mock the category or their interlocutor (see, for instance, Example 3.11 on page 95). The pupils laughed in response to the joke and joined in with the teacher's friendly banter of Meryem. Mr. de Jong's categorical joke functioned well as it adhered to an important convention of categorization among these pupils (described in Chapter 4), namely that people categorized as Nederlander did not usually mock other categories. Mr. de Jong's joke is categorically self-deprecatory, as he would be categorizable as a tatta himself, and he thus does not insult anyone else's category. In sum, in this example, the teacher can be seen to recruit pupils' localized meaning constructions to engage in jocular mockery.

\subsection{Conclusion}

In this chapter, I analyzed ethnic categorizations in interactions between pupils and teachers. I showed that many teachers problematized pupils' selfcategorizations as 'foreigners,' 'Moroccans,' or 'Turks' in interaction with me and with pupils themselves. This sometimes appeared to stem from their interpretation of those labels as indicating a lack of belonging, and at other times, from the labels' potential associations with negative characteristics, such as being a nuisance. As such, teachers' categorizations and their reactions to pupils' self-categorizations evoked conventional meanings of Marokkaan, buitenlander and Turk that echo societal discourses surrounding these categories, for instance that they indicate a lack of belonging to the Netherlands, or that they are used to stigmatize others. Pupils themselves shifted between, on the one hand, playful uses of category labels with those (negative) associations that are more conventional in wider societal discourse, 
and on the other hand, categories with their own, localized associations (such as being 'cool,' or being relaxed about rules, as analyzed in Example 5.1 and as seen in Chapters 3 and 4). In some interactions, such as in Example 5.2 and 5.5 , teachers' disagreement with pupils' self-categorization could also be understood as having a function in interaction, for instance to reinforce authority or discipline a pupil.

Teachers seemed to struggle with the apparent contradiction that they preferred their pupils with a migration background to self-categorize as 'Dutch,' but at the same time, they knew that there were differences between pupils with and without migration background which were furthermore emphasized by the pupils themselves. Teachers could also be seen to engage in discourses that positioned their pupils with and without migration backgrounds as categorically different. At least a part of the process of othering that some teachers engaged in by categorizing their pupils may be attributed to the diverging associations that teachers and pupils seemed to have with the labels 'Turk,' 'Moroccan,' 'foreigner' and 'Dutch.' Where pupils shifted between, and sometimes merged, their localized meaning constructions of those categories on the one hand, and more conventional, wider-spread uses of those categories on the other, teachers showed understandings of categories in terms of their conventional, wider-spread associations.

This chapter does not aim to suggest that it is per definition bad or irresponsible for teachers to use the labels their pupils use. The attunement to pupils' labelling practices as demonstrated by the teacher in Example 5.8, for instance, may have positive effects on mutual relations. What I argue, instead, is that it seems unlikely that pupils will feel (more) included when their teachers problematize the labels 'Moroccan, 'Turk,' or 'foreigner,' or urge their pupils to label themselves 'Dutch,' while they - and society at large - also position them as are categorically different from people without migration backgrounds. When teachers reject labels that pupils can hardly escape from, or that they have reappropriated, they might be seen to imply that being 'Dutch' is better. Stimulating a sense of inclusion for all pupils might be more effective when using categories that are not already subject to local renegotiation. 


\section{The use of linguistic resources in relation to categorization practices}

\section{Field notes, 2 March 2018}

"Say wollah?" Dounia sounds surprised when I tell her that this is my last week of fieldwork. "Wollah," I reply. She laughs, even though - or maybe because - this is exactly how pupils swore the truth of their statements to one another. "That's too bad, it was nice having you around."

This was the first time that someone said 'say wollah' to me - and I knew how I was supposed to answer! It feels as a kind of initiation ritual into a new level of intimacy. Unfortunately, it's one of my last days at school and somehow, I feel sadder about saying goodbye to the pupils. I remember how, during the first month that I spent at South High School, the pupils and I had trouble understanding each other. They found it strange to have an adult in class who did not act as an authoritative figure, who was there voluntarily ("So you can leave whenever you want? Why are you still here!?"), and just sat with them during their classes to learn things about 'their language.' We also struggled to understand each other in the most literal sense of the word: We spoke differently. Even when we all spoke Dutch, our Dutch had such different sounds to it that it took our ears some time to adjust to one another. And then there was dialect, and Turkish, and lots of expressions and words that were unfamiliar to me: skeer, mashallah, safi, sies, hayek...

\subsection{Introduction}

The previous chapters have all dealt with aspects of practices of ethnic categorization in class 3/4b: Pupils' negotiations of the meanings of ethnic categories (Chapter 3), the interactional functions of references to ethnic categories (Chapter 4), and teachers' role in categorization practices (Chapter 5). In the current chapter (Chapter 6), I examine the relations between categorization practices and the use (and policing) of different linguistic resources in South High School. I analyze multilingual behavior among the pupils of class $3 / 4 \mathrm{~b}$, the frictions this caused with the monolingual language policy, and the ways in which teachers as well as pupils negotiated those frictions.

As the opening vignette illustrates, the pupils of class $3 / 4 \mathrm{~b}$ engaged in linguistic practices that I was not familiar with before I met them. They had a wide range of linguistic backgrounds and competencies, which aside from 
standard Dutch ${ }^{27}$ included dialects spoken in Limburg (particularly Venloos and Tegels), Turkish, Moroccan Arabic, and Berber (and some other languages). Most pupils also had some knowledge of English. Pupils' multilingual repertoires found their way into the classroom in the form of full conversations, insertion of sentences, or words, or they were hinted at in certain patterns of pronunciation. Their linguistic practices existed in friction with South High School's language policy, however, which prescribed the use of standard Dutch only.

South High School's Dutch-only policy was not presented explicitly. There were no signs or posters telling pupils what language (not) to speak (although there was a poster against swearing), and there were no explicit instructions or policy briefs for teachers about language rules. It was taken for granted that everyone at school knew that the only official language of instruction and communication was standard Dutch. All pupils were able to communicate in standard Dutch without any problems (although many teachers frequently corrected what they considered pupils' 'ungrammatical' standard Dutch utterances ${ }^{28}$ ) and this was the language that they used most often. Several other languages also found their way into the daily school context, however. This chapter focuses on Limburgish, Turkish, Arabic and Berber. Other languages, like English, also had a prominent position in the pupils' linguistic repertoire. English occurred in the form of music lyrics sung

\footnotetext{
${ }^{27}$ The term 'standard Dutch' is often used in reference to a variety that is perceived as 'neutral' or 'without accent' (Smakman 2006). Dutch as it is spoken by most Limburgish speakers, including the pupils of class $3 / 4 \mathrm{~b}$, is usually viewed as 'accented,' and might thus not be perceived as 'standard' by speakers from other parts of the Netherlands. Throughout the chapter, I nonetheless use the term 'standard Dutch' to refer to the variety spoken by the pupils and teachers because the speakers thought of it as such, and because it clarifies the distinction between uses of (what they perceived as) 'standard Dutch' and 'dialect.'

${ }^{28}$ For instance, many pupils used bun - the third person plural pronoun for object position (similar to English 'them') - in the subject position instead of the standard subject-form $z i j$ or ze. The use of bun in the subject position is widespread, though it also "provokes strong feelings of abhorrence among the gatekeepers of a correct standard Dutch" (van Hout 1999). Teachers often corrected the pupils when they used it, and other teachers did so themselves. Another common linguistic practice that teachers corrected was the use of the definite determiner de where the definite determiner het is required, or non-standard die instead of dat. Young people have been reported to use these 'incorrect' forms on purpose, however (e.g. Cornips 2008; Nortier and Dorleijn 2008).
} 
in class, or common expressions inserted into Dutch utterances (for instance "that's it" or "fuck you"). Whereas Limburgish, Turkish, Arabic and Berber were distributed among pupils in particular ways, however, English was used by all pupils and did not show relations to pupils' categorizations. As this chapter focuses on the relation between the use of linguistic resources and ethnic categorizations at school, the use of English will thus not be part of the analyses in this chapter.

The examples in this chapter will show that, in general, the teachers of class $3 / 4 \mathrm{~b}$ reacted much more strongly when pupils spoke Turkish, Moroccan Arabic, or Berber, than when pupils used dialect. At the foundation of this were pervasive language ideologies, such as the idea that languages belong to people, who belong to places (Karrebæk 2013; Quist 2010). This results in the idea that languages, and their speakers, can be seen to be 'in' or 'out of place' (Thissen 2018). This symbolic organization of languages and their speakers in this classroom reflects and reproduces wider patterns of social stratification as they have been analyzed in the previous chapters (Collins 2017; Nørreby and Madsen 2018).

Identifying and naming languages and dialects - as I do in this chapter suggests a view that has been widely challenged in sociolinguistics, namely one in which each 'language' is portrayed as a separable entity (Jørgensen et al. 2011; Makoni and Pennycook 2005). Languages as bounded 'units' may not describe "any real state of affairs in the world" (Makoni and Pennycook 2005, 147), but as is apparent from participants' practices of identifying and naming languages, they are a social and psychological reality for speakers (Cornips et al. 2016). Because I analyze such ideologies in this chapter, I refer to languages the way pupils and teachers did, that is, as if they were separate and bounded entities.

The chapter is organized as follows. Section 6.2 provides some context to the position of Limburgish and Turkish, Moroccan Arabic and Berber in the Netherlands, and particularly in education. Section 6.3 contains the data analysis, divided into three parts. The first of those parts gives an overview of which languages the pupils used, and the context and manner in which they did, including their local indexicalities. The second part (6.3.2) analyzes teachers' policing of those languages, and the third (6.3.3) shows how teachers and pupils creatively dealt with, challenged, and negotiated the language policy in practice. The chapter finishes with a discussion of how ethnic categories and uses of linguistic resources at South High School were part of an interrelated system of categorization, by which pupils and teachers produced, reproduced, challenged, and negotiated local and wider societal social hierarchies. 


\subsection{Context: Languages and their positions in the Netherlands}

In order to understand the distribution and use of utterances associated with languages other than standard Dutch among the pupils and teachers of class $3 / 4 \mathrm{~b}$, and the reactions these languages evoked, it is first necessary to know more about perceptions of these languages in and outside educational institutions in the wider societal context of the Netherlands. To that end, this section first discusses Limburgish, and thereafter, Turkish, Moroccan Arabic, and Berber.

\subsubsection{Limburgish}

In educational institutions, Limburgish has always been relegated to the fringes. The use of one 'national' language in a standardized education system has been deemed pivotal in processes of nation forming since the Enlightenment (Kroon and Vallen 2004). In the Netherlands, this has meant that during the rise of a unified schooling system, standard Dutch was implemented as the language of schooling, and anything else was considered 'uncivilized' - a position which is reflected until today in the term $A B N$ or Algemeen Beschaafd Nederlands 'General Civilized Dutch,' often used (by nonlinguists) to refer to standard Dutch (an alternative preferred, for instance, by linguists, is Algemeen Nederlands 'General Dutch' or Standaard Nederlands 'Standard Dutch'). The notion of languages being civilized (i.e. standard Dutch) versus uncivilized (i.e. dialects) was dominant in education until the 1970s (Kroon and Vallen 2004). Aside from being perceived as uncivilized, dialects were seen to imply educational disadvantage. In Limburg, a shift in this perception occurred when the well-known 'Kerkradeproject' of 1973 found that there was no relation between children's school performance and speaking a Limburgian dialect. The project showed that differences between dialect-speaking children and non-dialect-speaking children could be attributed to teachers assessing dialect-speaking children more negatively than children who spoke standard Dutch (Kroon and Vallen 2004). The findings of this project were disseminated in schools, and since then, schools and educators have tended to have somewhat more positive (or at least less negative) attitudes toward Limburgish.

Limburgish never acquired an official position in education, however. Although it has been officially recognized as a regional language since 1997, this does not come with any obligations on the part of the Dutch state or the Province of Limburg to provide financial support or to develop policies regarding its position in education (Camps 2018). The medium of instruction is standard Dutch in all levels of education, from pre-school to secondary education (with the exception of some Dutch-English or Dutch-German 
tracks). In Example 6.1, two pupils of class 3/4b who attended primary school in Tegelen - a town near Venlo - confirmed the status of dialect as relegated to the home context. However, in their experience, that division between home and school language policy had not been strictly reinforced. In my question in line 1, 'there' refers to the primary school that these pupils had attended.

Example 6.1. 30 May 2017. Care and Well-being class. Participants: Eva(E), Sanne (S), and the researcher (Pomme, $P$ in the transcript).

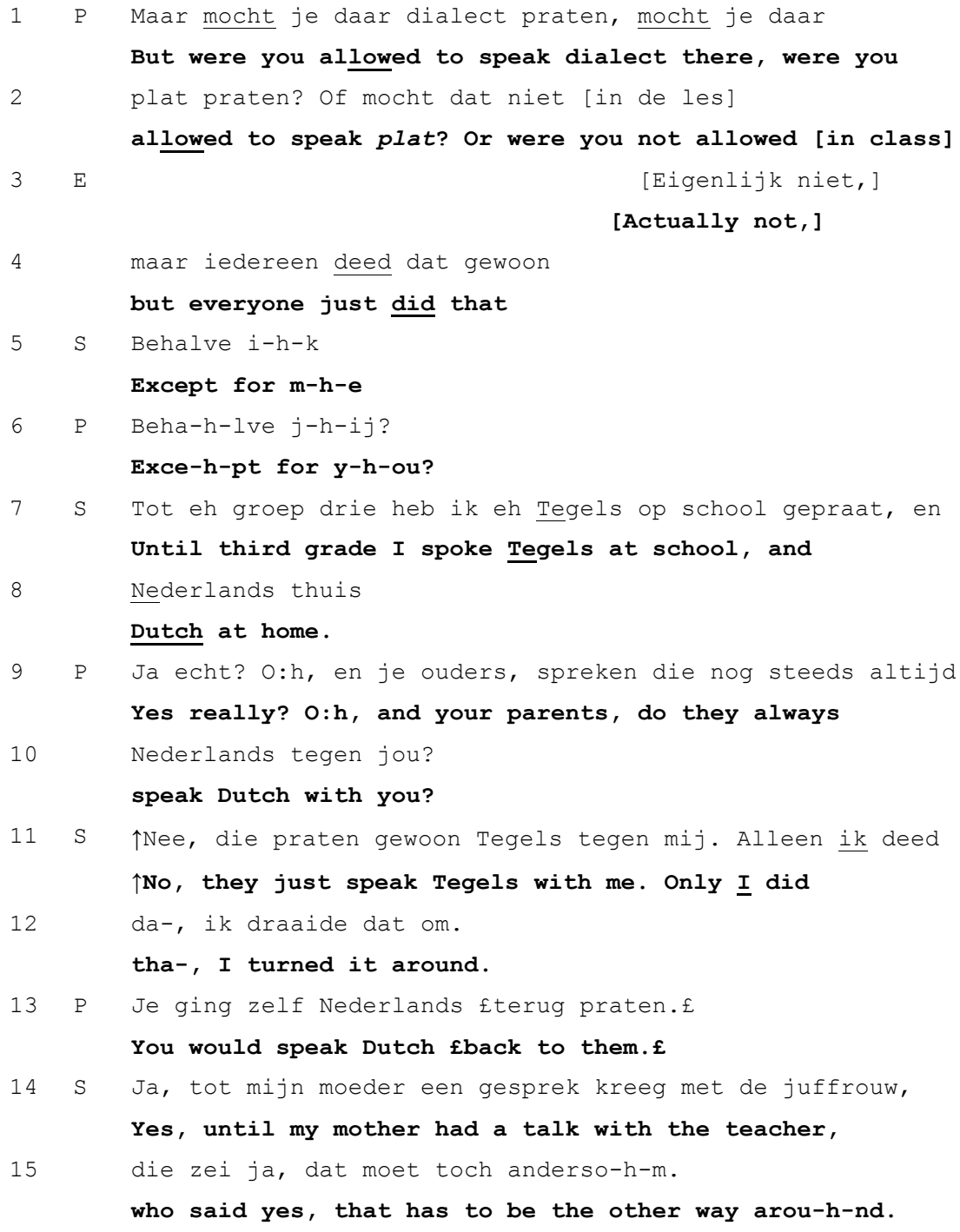


In this short conversation, Sanne and Eva construct the home as the logical context for speaking plat (line 2) or, using another term, Tegels (line 7), and school as the context where (standard) Dutch should be spoken - even if this rule was not reinforced very strictly in their case. With our laughter and smile voice in lines 5-6 and 13-15, Sanne and I construct her use of Dutch at home, and Tegels at school, as a somewhat strange or notable reversal of what is expected. Indeed, such language practices are notable as it has been reported that many children stop speaking dialect altogether from the moment they enter pre-school and are confronted with a Dutch monolingual language policy (Cornips, 2020b).

Sanne and Eva's experience of not being supposed to speak Tegels at school, without this being strictly enforced, also confirms observations, however, that dialect is not explicitly forbidden but that it finds a place in informal, intimate, non-plenary moments in the classroom - especially in educational institutes for younger children (Morillo Morales 2017). The negative perceptions of dialect as related to educational or societal disadvantage do not appear to be very prominent anymore. Even if dialects suffer from negative stereotyping by speakers from other parts of the Netherlands (Cornips and de Rooij 2018a), it is seen much more positively in Limburg than it is in many other places (Kroon and Vallen 2004). The societal concern around 'civilization' in relation to language seems to have waned, and with it the attention to which languages do (not) index civilization (see also Duchêne and Heller 2012). Instead, debates surrounding 'identity' and 'belonging,' and languages as indexical of those concerns, have gained prominence in particular in the context of increasing immigration into the Netherlands, the associated linguistic, cultural, religious, ethnic diversities, and related concerns about 'nationhood' and the meaning of 'citizenship' (Duyvendak 2011). The next section illustrates the role of languages in those debates.

\subsubsection{Turkish, Moroccan Arabic, Berber}

In the 1960s, when post-war economic growth caused a need for semi- and unskilled labor, large scale Turkish and Moroccan migration to the Netherlands started (Bouras 2013). The incoming 'guest workers' were expected to leave after a while, but in the 1970s it became clear that the migrants were settling with their families. During these initial years of Moroccan and Turkish presence in the Netherlands the government stimulated migrants to maintain ties with the 'home country,' initially because it was supposed to make their return easier, and when the government 
acknowledged that the migrants had settled, because it was thought to enhance their social and cultural emancipation (Bouras 2013).

Part of the stimulation to maintain ties occurred through language education: From 1974 to 2004, different government programs existed that funded classes in migrant languages (Extra and Yağmur 2012). Under the acronym OETC (Onderwijs in Eigen Taal en Cultuur, 'Instruction in one's own language and culture'), such classes formed part of the regular curriculum for children of migrant backgrounds. Their purpose was to "teach pupils the culture and the official, national standard language of the country of origin" (Bezemer and Kroon 2006, 16). This usually excluded teaching non-official 'own' languages such as Berber, but also national varieties such as Moroccan Arabic (instead of standard Arabic). In the first years, these classes were meant to be of use to children when they would return to their country of origin; later, from 1979, the idea was that it would help them with their 'integration' in the Netherlands (Bouras 2012, 89). There were several difficulties with the implementation of OETC, however. Among other things, there were not enough teachers or materials. In 1998, migrant language education was taken out of the regular curriculum and continued as OALT (Onderwijs in Allochtone Levende Talen, 'Instruction in non-indigenous, living languages'). This meant that migrant language teaching became an extracurricular provision which was aimed at teaching children's supposed 'mother tongue' (again, not if that happened to be Berber, Sranan, Papiamentu, or another 'unofficial' language), but it could also be used as a means to support pupils in learning the regular school curriculum or learning Dutch (Bezemer and Kroon 2006). In practice, the majority of OALT funding was used for 'language support' - which could imply using the 'mother tongue' to support teaching the regular school curriculum, to help teach Dutch, or to teach the 'home language.' Because schools were uncertain about the goals and guidelines of this program, different municipalities and schools implemented it differently (Bezemer and Kroon 2006).

Around the turn of the century support for these programs had dropped significantly. One of the major underlying motivations for OALT was that if children learned 'their own' language, it was thought to help them acquire Dutch. A report by the Education Council (Onderwijsraad) in 2001 did not find such effects, however, and one of the main motivations behind the program was thereby proven untenable. Furthermore, the general sociopolitical tide had turned toward an increasingly negative stance toward immigration and people with migration backgrounds. Languages other than Dutch came to be seen as a "major underlying barrier to educational success" (Extra and Yaĝmur 2006, 53) and as a barrier to integration, and in 2004 the 
government ended all funding for teaching migrant languages. Today, secondary schools may offer Turkish and Arabic as school subjects, but not many schools do because of this lack of funding. In 2011, for example, only 0.13 percent of all pupils took their exams in either Turkish or Arabic (Extra and Yağmur 2012).

A number of studies have demonstrated that the idea that other home languages are a barrier to educational success remains prominent among many teachers. Spotti and Kroon $(2009,179)$, for instance, found that "the identities of immigrant minority pupils are constructed, in the class teacher's discourse, on the basis of language attributions that find their pivotal point in ideologies of language disadvantage provoked by the lack of Dutch language skills on the part of these pupils' parents." Similar findings are described by Bezemer (2003, 2007) and Spotti (Spotti 2014b) in Dutch primary schools. In Belgium, with its similar history of immigration, Agirdag $(2010,307)$ describes how a secondary school imposed Dutch monolingualism, while the Turkish of Turkish-Dutch bilinguals in secondary school was mostly perceived as "a barrier to educational and occupational success." In many cases, strict language policies that prescribe the use of the national standard language are justified by the idea that they will 'improve pupils' language,' and that this is the necessary pathway to educational and occupational success (e.g. Jaspers 2017; Karrebæk 2013).

Pupils are not passive recipients of such doctrines, or mere victims of linguistic discrimination, however, as Jaspers (2005a, 2011b, 2011a, 2014) has shown extensively, and neither are teachers mere 'soldiers of the system' who implement strict language policies without fail (Jaspers 2019). Pupils as well as teachers negotiate language policies in practice, for example by playfully breaking them, protesting, reproducing, ignoring, or making fun of them. As will be illustrated in Section 6.3.3, this was also the case for the pupils of class 3/4b.

These short backgrounds of Limburgish as compared to Turkish, Moroccan Arabic and Berber explain much of the ideologies that emerge from the data presented in the following paragraphs. Although, strictly spoken, none of those languages had an officially ratified place in the linguistic environment of South High School, they all found a way into daily life at school. As described in the following paragraphs, the differences in reactions to those languages by teachers was striking, however.

\subsection{Language use and ideologies in class $3 / 4 b$}

In this section, I first give an overview of how and when pupils used languages other than Dutch in the classroom, and what use of those languages indexed. 
Thereafter, I focus on the reactions that pupils received when speaking those languages, mostly from teachers, but also from other pupils. In both sections, I first address Limburgish, and thereafter, Turkish, Arabic and Berber ${ }^{29} \mathrm{I}$ treat those latter three together because teachers did not distinguish between them in their policing. In the final data analysis section (6.3.3), I describe ways in which teachers and pupils creatively dealt with these linguistic restrictions.

\subsubsection{Which languages were spoken, and in what contexts?}

Limburgish did not have a prominent place in daily school life as I observed it in class 3/4b. Many pupils (an estimated 13 out of 37) seemed to speak it, and on occasions, I heard them use Limburgish with each other. This usually occurred when they engaged in private conversation. I observed some instances where pupils spoke Limburgish with teachers, but this happened very rarely and usually in short, informal exchanges. More often, they could be observed using standard Dutch in class. Some pupils told me that their parents wanted them to speak dialect at home, but that they did not do so. As I wrote in a field note, "They seem to find dialect ugly or stupid" (Field notes, 8 February 2017). Not all pupils had such a distinctly negative attitude toward Limburgish, and for many it seemed very normal to speak it together. It was not a variety that had distinct social prestige among the pupils, however.

I only heard pupils with a Dutch background, that is, without a migration background, use dialect in interaction among each other. When I asked pupils with migration backgrounds whether they spoke dialect, they responded with laughter. In the interaction in Example 6.2, which occurred during an informal conversation in class, I asked Yildiz and Dounia, who had a Turkish and Moroccan migration background, respectively, whether they spoke dialect.

Example 6.2. 15 May 2017. Participants: Yildiz (Y), Dounia (D), the researcher (Pomme, $\mathrm{P}$ in the transcript). Care and Well-being.

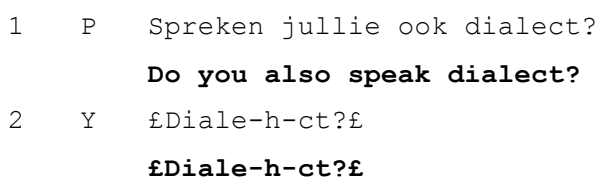

\footnotetext{
${ }^{29}$ The majority of Moroccan migrants to the Netherlands came from the Rif and Sous areas, where several varieties of Berber are spoken (Tarifit, Tashilhit, Tamazight) (Bouras 2012). Migrants from other parts of Morocco mostly speak Moroccan Arabic (Darija).
} 


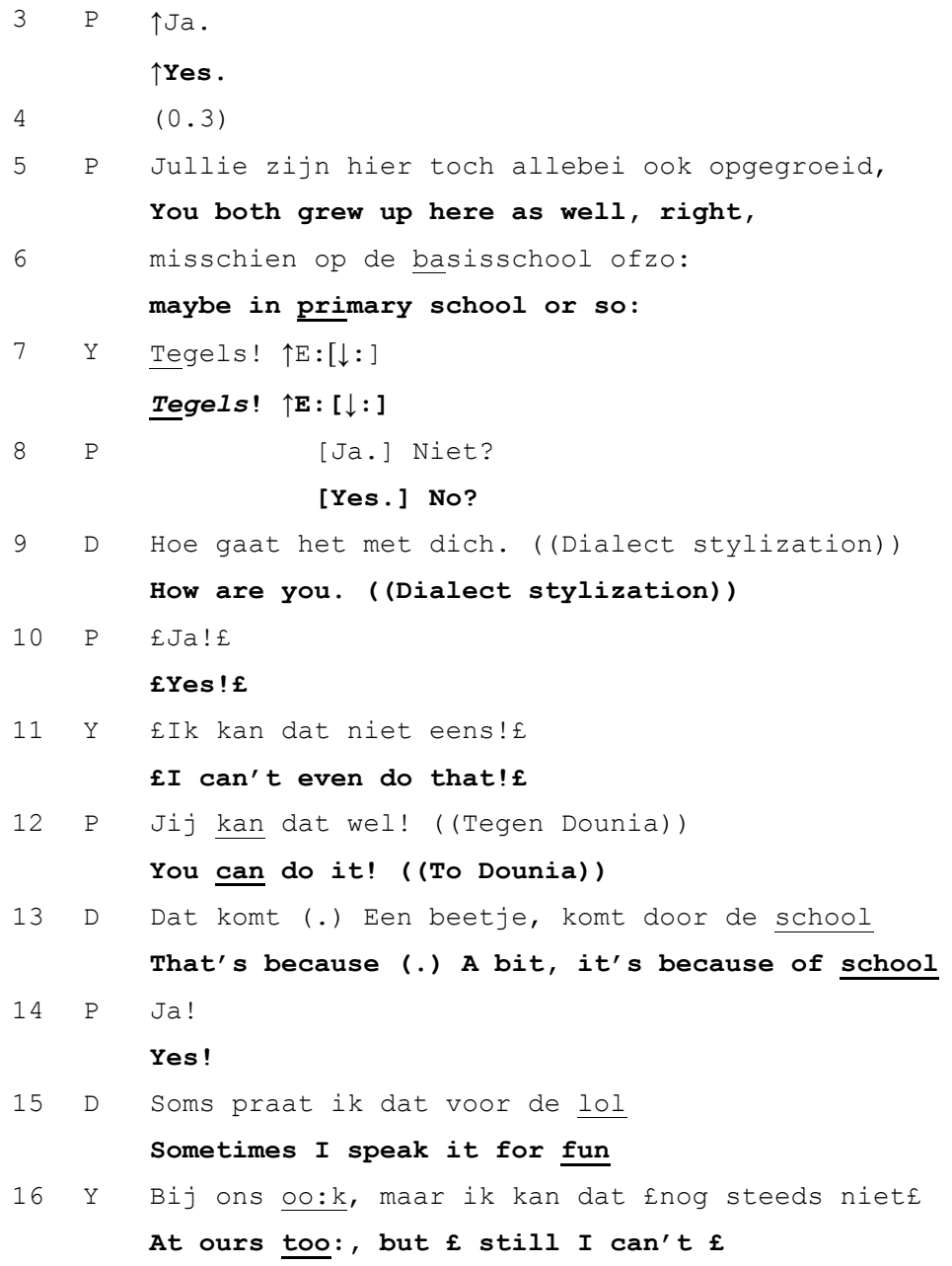

Even though my question in line 1 does not contain elements that invite a laughing response (Jefferson 1979), e.g. I am not smiling and the turn does not contain laughter particles, Yildiz's laughing response in line 2 "acts as an implicit commentary on the question, undercutting its legitimacy as a question to be taken seriously" (Romaniuk 2013, 205). Her repetition of the last word of my question points to the laughable element in that question, that is, the suggestion of her speaking dialect. I do not join in with her laughter, but after a short silence, I expand on my question in lines 5-6, offering a justification, but Yildiz merely utters the name of the dialect that I might be suggesting she speaks, i.e. Tegels. This is, again, produced in a way that suggests that the idea is preposterous. In line 8, I insist on the yes/no interrogative first posed in line 1 , but Yildiz actually never produces a type-conforming response by 
confirming or denying (Raymond 2003), even though her laughter implicates a clear "no" (Romaniuk 2013).

In line 9, Dounia responds to the preceding exchange by producing a dialect stylization, which most prominently consists of the dialectal form dich instead of the standard Dutch jou or je and a marked intonation pattern. Again, this is not a type-conforming response (Raymond 2003), although I respond to it as an implicit 'yes' in line 12. Dounia explains that she speaks some dialect because of her (primary) school, and that sometimes, she speaks it 'for fun' (line 15). Like Yildiz, Dounia implies that for her it is not a serious possibility to speak Limburgish. Although these pupils had been born in Limburg and had grown up around speakers of Limburgish, pupils seemed to associate Limburgish with the people they referred to as Nederlanders or 'Dutch people.' ${ }^{30}$ The few times that I heard pupils with a migration background use dialect, it was in a stylization (e.g. Field notes, 24 January 2017; 8 May 2017). This reinforced the image of Limburgish as a variety that is inauthentic when spoken by people with migration backgrounds (see Cornips 2020a) because stylizations project "strategic inauthenticity in selfprojection" (Coupland 2011, 155). These pupils' ideology of Limburgish being reserved for people without migration background is probably not only the result of their self-exclusion from this language. In Limburg, people who are perceived to 'not look Dutch,' who in other words are racialized, are rarely addressed in dialect, even if they do speak it (Cornips 2020a; Thissen 2018). Socialization into the idea that only people without migration backgrounds speak Limburgish occurs from a very early age: It has been observed that already in pre-schools toddlers with migration backgrounds are addressed in standard Dutch rather than dialect, while toddlers of Limburgian families are individually addressed in dialect (Morillo Morales 2017).

Although 3/4b's pupils with migration backgrounds did not use Limburgish, their Dutch sounded markedly 'local' to me in some other ways. Like pupils without migration background, they sometimes used expressions associated with Limburg such as "sies" ('honey') or "ocherm" ("poor thing') and they had the 'melodious pronunciation' and so-called 'soft g' ([y]) that are commonly associated with how standard Dutch is spoken in the South of the Netherlands (e.g. Thissen 2013). They were aware that those elements marked them as 'Southern' in the eyes of speakers from the center of the Netherlands. Khadija, for example, who had a Moroccan migration

30 As mentioned in Chapter 3, the pupils who most frequently engaged in categorization referred to people from Limburg as Nederlander, without distinguishing them from Nederlanders from other parts of the Netherlands. Dialect was thus constructed as for Nederlanders - not for 'Limburgers.' 
background, remarked: "You know what I hate? I hate people from The Hague. When they hear a soft g, they always start to imitate some sort of accent" (Field notes, 19 May 2017).

Most teachers at South High School spoke Limburgish (aside from standard Dutch) and often used it during breaks in the teachers' lounge. Teachers and others present in the break room who did not speak a dialect like me - were in the minority and were addressed in standard Dutch. As one teacher explained the language policy to me, teachers were allowed to speak dialect with each other during breaks but were not supposed to do so when they went to see each other in class (Field notes, 9 January 2017). Teachers and pupils were also supposed to speak standard Dutch together, but in my observation did not always adhere to this rule: sometimes they had short informal exchanges in dialect (as one pupil mentions in Example 6.6 later in this chapter). The one time that I witnessed Limburgish being used in a pseudo-official, plenary context was when an announcement was made through the school intercom in which all pupils were invited to celebrate the Carnival event De Boerebroélof ('The farmers' wedding'), in a partially dialectal, partially standard Dutch utterance (Field notes, 13 February 2017). Carnival is often associated with role reversals and temporary suspensions of the social order, and in Limburg, this popular festivity is closely associated with the use of Limburgish, reproducing a view of the dialect as indexical of 'localness' (Cornips, de Rooij, and Stengs 2012; Cornips and de Rooij 2015). These conditions made the use of Limburgish appropriate in this specific plenary context, through the intercom.

In some ways, Turkish, (Moroccan) Arabic and Berber had a stronger presence among the pupils of South High School than Limburgish. The pupils with a Turkish migration background of class $3 / 4 \mathrm{~b}$ all had conversational proficiency in Turkish, and frequently spoke Turkish together outside of class, and in class when they (thought they) were outside of the teachers' earshot. Sometimes, Turkish was used to say something that was designed to not be understood by a teacher, as in Example 6.3.

\section{Example 6.3. Field notes, 15 December 2017.}

Care and well-being class. Half of the class is sitting at the table, eating the dish they prepared as an assignment. I am standing nearby. The rule is that the pupils who have cooked sit down together afterwards at a long table to eat what they have prepared. Those who have not cooked are not allowed to eat (or even taste) what their peers have made. Meryem, who has cooked and is eating, tells her friend Yildiz (in Dutch) to come eat as well. It appears that Meryem hasn't noticed that the teacher is sitting with them at the same long table, and Yildiz has not cooked a dish so she's not allowed. Yildiz replies to Meryem in Turkish. From context, I deduce 
that she is telling Meryem that the teacher is sitting right there. A short exchange (in Turkish) follows. Right after, Meryem catches me watching them and smiles somewhat guiltily. The teacher doesn't comment on the exchange (which occurred while a lot of other pupils were speaking simultaneously).

In this example, Meryem and Yildiz used Turkish to communicate something that they did not want the teacher to hear. Indeed, the teacher did not react. The example shows that pupils used Turkish rather fluently and, sometimes, in class, even though they knew they were not supposed to.

I rarely observed pupils using Moroccan Arabic or Berber with each other in any way that exceeded a couple of words or a single phrase. Several pupils with a Moroccan migration background mentioned to me that they did not speak 'Moroccan' well (it was not always clear to me what this label, which they often used, referred to - Berber or Moroccan Arabic). This confirms findings of other studies that youth with a Turkish migration background use Turkish much more often than youth with a Moroccan migration background use Moroccan Arabic or Berber (Extra and Yaĝmur 2010). ${ }^{31}$

Daily speech of all pupils - with and without migration backgrounds included many elements that are associated with Turkish, Moroccan Arabic, Berber, and sometimes other Dutch immigrant languages like Papiamentu. Dutch as spoken by youth with migration backgrounds has been described extensively (e.g. Cornips 2002; Mourigh 2019; Nortier and Dorleijn 2008) and it has been observed that this style is not restricted to youth with migration backgrounds (Cornips, Jaspers, and de Rooij 2014; Jaspers 2008). At South High School, it was common for pupils to use elements from languages that were not associated with their own (lack of) migration background (i.e. to engage in "crossing," see Rampton 2005). Pupils with - and also some without - migration backgrounds often used words such as wollah ('I swear'), wollah meh ('I swear' in a negation), 'kifesh' ('how' or 'why'), wahed ('a' or 'one') or yalla ('let's go'). They also frequently deleted articles, and produced [sx] as [šx] (Nortier and Dorleijn 2008). I regularly heard pupils teach each other words and expressions in 'their' language. This occurred especially with Turkish, Berber and Moroccan Arabic (but not with Limburgish). This did not mean

${ }^{31}$ This may be explained (partly) by the low prestige of these languages among their speakers, as well as the fact that Arabic and Berber languages are mutually unintelligible. As Nortier and Dorleijn $(2008,139)$ explain: "The sociolinguistic position of Moroccan languages is totally different compared to Turkish. Both Berber and Moroccan Arabic have a low prestige among their speakers (with the exception of an increasig [sic] group of Berber language activists). Berber and Moroccan Arabic are not mutually intelligible, neither of the two has a written tradition or a specific standard variety." 


\section{Nederlanders and buitenlanders}

that linguistic forms associated with those languages were a 'free-for-all,' however (cf. Blommaert and Rampton 2011). In the interaction below, a number of pupils with migration backgrounds told me that it bothered them when pupils without migration backgrounds used 'their' language. This exchange occurred just after the exchange transcribed in Examples 3.9 and 3.10 (Chapter 3, pages 89 and 93).

Example 6.4. 16 June 2017. Participants: Meryem (M), Yildiz (Y), Amira (A), and the researcher (Pomme, $\mathrm{P}$ in the transcript).

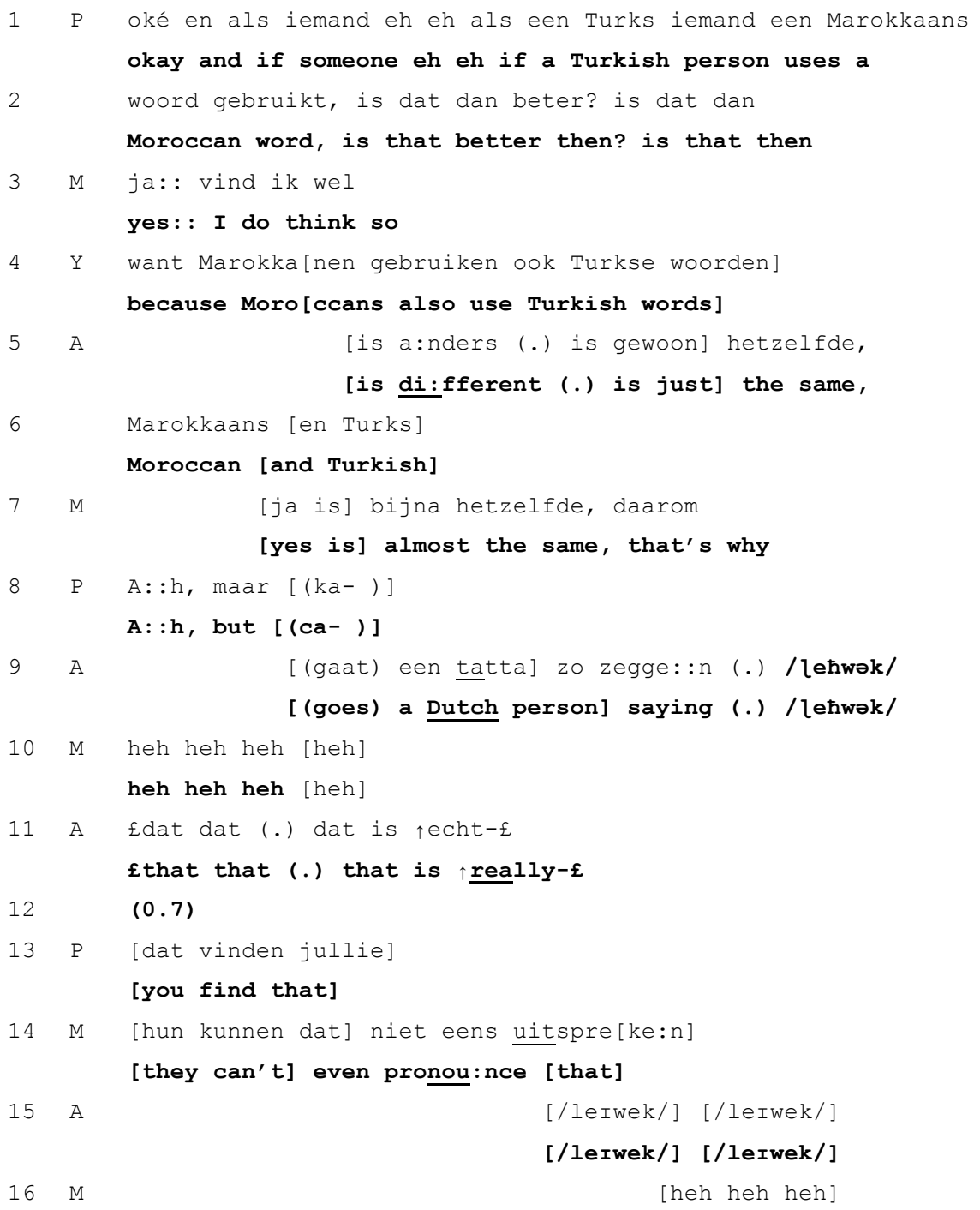




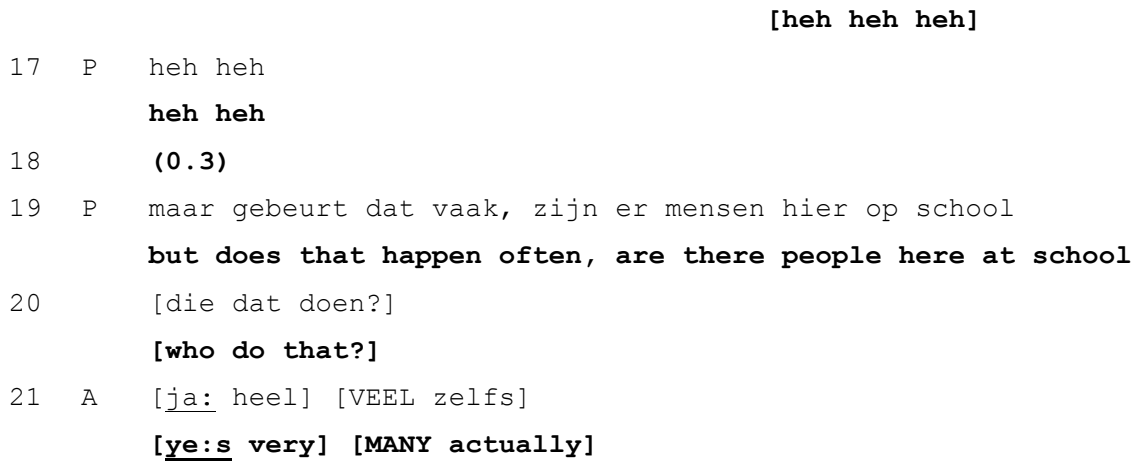

In Example 6.4, Meryem, Yildiz and Amira make fun of people they refer to as tatta's (another word for people perceived as not having a migration background, see Chapter 3 for a background of this term) who use words associated with Turkish or 'Moroccan.' In lines 9-16, they laugh about mispronunciations. ${ }^{32}$ "They ruin your language," Amira said a little while later during the interview. It is less of a problem, in their eyes, if 'Turkish' people use 'Moroccan' words, or vice versa, however. As they argue in lines 5-7, 'Turkish' and 'Moroccan' languages are similar enough. Considering that Turkish, Berber, and (Moroccan) Arabic are linguistically different and mutually unintelligible, it seems likely that this perception does not stem from a comparison of the features of those languages, but instead, that it has to do with how pupils treated ethnic categories. They endowed the categories Marokkaan and Turk with many of the same associated characteristics and grouped these two categories under the umbrella term buitenlander. They often emphasized the opposition between buitenlanders and Nederlanders. In Example 6.4, it appears that that opposition is transported to perceptions of differences and similarities between languages.

As remarked by Amira in line 21, many pupils without migration backgrounds in class 3/4b used words associated with Berber, Moroccan

${ }^{32}$ The word that these pupils are laughing about in lines 9 and 15 can be written as 'li7wek' in Latin alphabet. Around the time of this extract, it seemed to be a favorite word of these pupils. They explained it to me as part of a joke, where one pupil would ask: "Do you know him?" And then the other pupil says, "who?", and the first pupil replies "li7wek." One pupil (Amine) told me it is like saying "April fool's"; another pupil (Dounia) said it was just a random name. My colleague, who speaks Moroccan Arabic, recognized it as a rather vulgar joke (though the pupils' use of it was incomplete), and hypothesized that the pupils might have been embarrassed to tell me the word's actual meaning (something like "fuck"). 
Arabic, or Turkish. These were the same words that pupils with migration backgrounds used most as well, for example wollah, tfoe, safi, mashallah, or salam aleykum. This linguistic practice carries associations with national and international urban youth culture and popular Dutch (often hip-hop and rap) music (see also Cornips and de Rooij 2013; Dorleijn, Mous, and Nortier 2015) and in this context seemed to index a 'cool,' young persona. Even though the pupils in Example 6.4 complain about pupils without migration backgrounds using 'their' language, I never witnessed any explicit policing of it happening. As a matter of fact, if there were any reactions at all, they were often rather positive. On one occasion, Nikki came into class and greeted Amira, Dounia and Meryem with a loud 'Salam aleikum!' (Arabic greeting). The girls smiled: 'Aleikum salam!' (Field notes, 24 March 2017).

\subsubsection{The enforcement of language policies in practice}

As mentioned, South High School had a rather inexplicit, taken-for-granted language policy that dictated the use of (what teachers considered to be) standard Dutch in class. During my time at the school there was no formal document detailing this language policy, and when I asked the school principal about it, he merely mentioned that pupils were supposed to speak standard Dutch. In practice, reinforcement of the standard Dutch language policy clearly distinguished between the different languages discussed previously.

Teachers hardly ever policed uses of Limburgish. Sometimes, they made a meta-linguistic comment, like in Example 6.5 below.

\section{Example 6.5. Field notes, 27 January 2017.}

Physics class, the teacher is explaining what phosphor is. "Phosphor is often used in...?" the teacher asks. "Lucifers ['matches']!" answers one pupil. "Correct, but especially in?" It's silent for a bit. "Zjwaegelkes ['matches' in dialect]?" tries a second pupil. "Well, that's the same thing, but then in... Tegelen?" - "Steyl!"

In this example, the first pupil answers the teacher's question in standard Dutch. The answer is correct, but it was not what the teacher had in mind and he poses the question again. A second pupil attempts to answer the teacher's question, but actually provides the same answer as the first pupil, but in dialect. The teacher then attempts to link this dialect word (zjwaegelkes) to a geographical origin, guessing that the term is from Limburgish as spoken in Tegelen. The pupil, however, is not from Tegelen but another nearby town called Steyl. This interaction illustrates the "ongoing process of enregisterment resulting in dialects that have become distinctive and characteristic for specific localities on a micro-level within the province" 
(Cornips et al. 2016, 191) and the high dialect awareness that accompanies it. It also shows how teachers commonly dealt with dialectal utterances in class. Here, the utterance is identified, acknowledged, and geographically located, and the class continues. On many occasions, teachers did not react at all to dialect use by pupils, or they responded to it (usually in standard Dutch) if it was addressed to them, or ignored it if it was spoken among pupils.

As a result, some pupils seemed uncertain about the do's and don'ts regarding the use of dialect. In Example 6.6, I asked Sanne and Eva (who also featured in Example 6.1) about the language policy at South High School. In this interaction, we refer to Limburgish as 'plat.'

Example 6.6. 30 May 2017. Participants: Sanne (S), Eva (E), the researcher (Pomme, $\mathrm{P}$ in the transcript). Care and Well-being class.

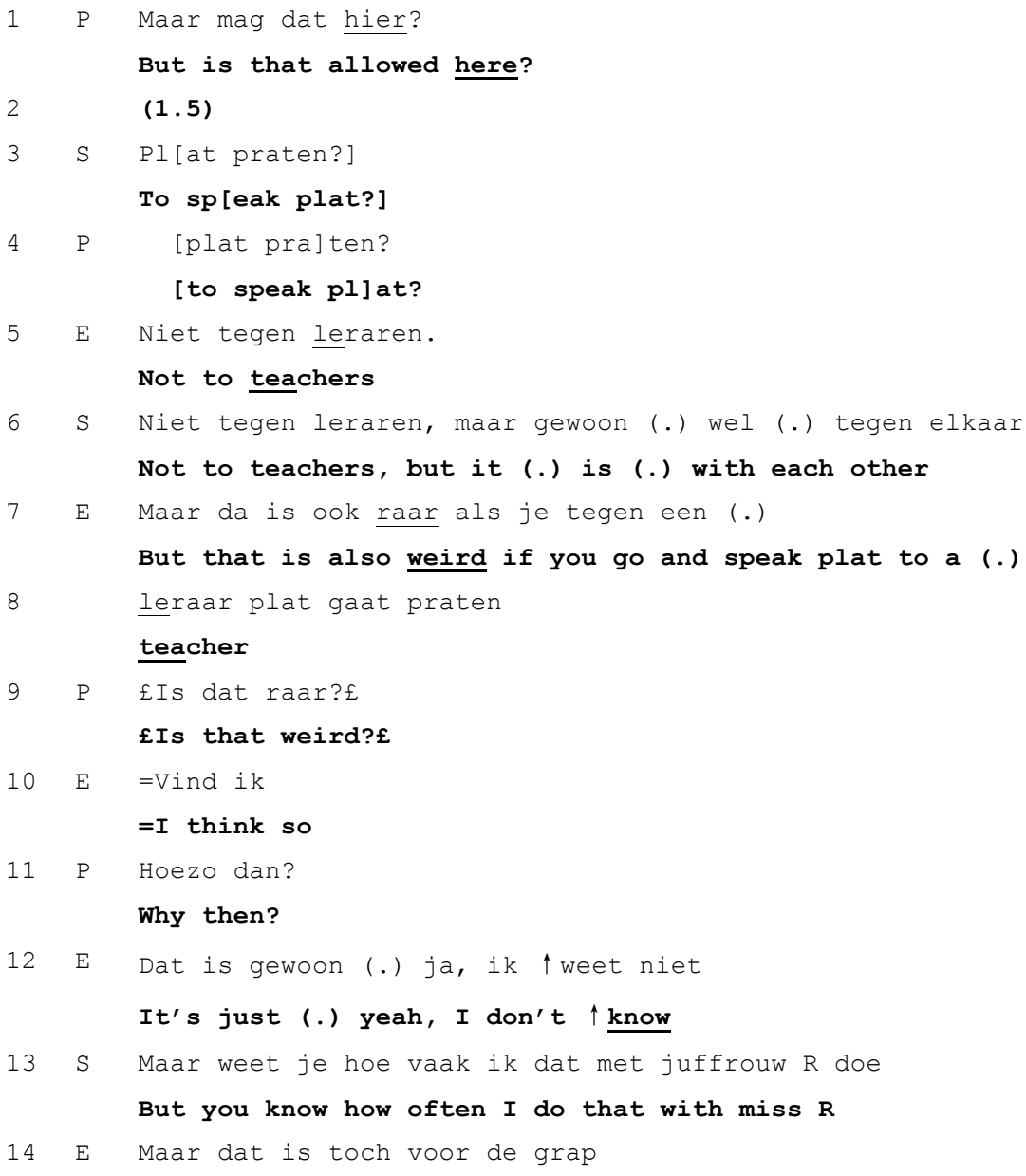




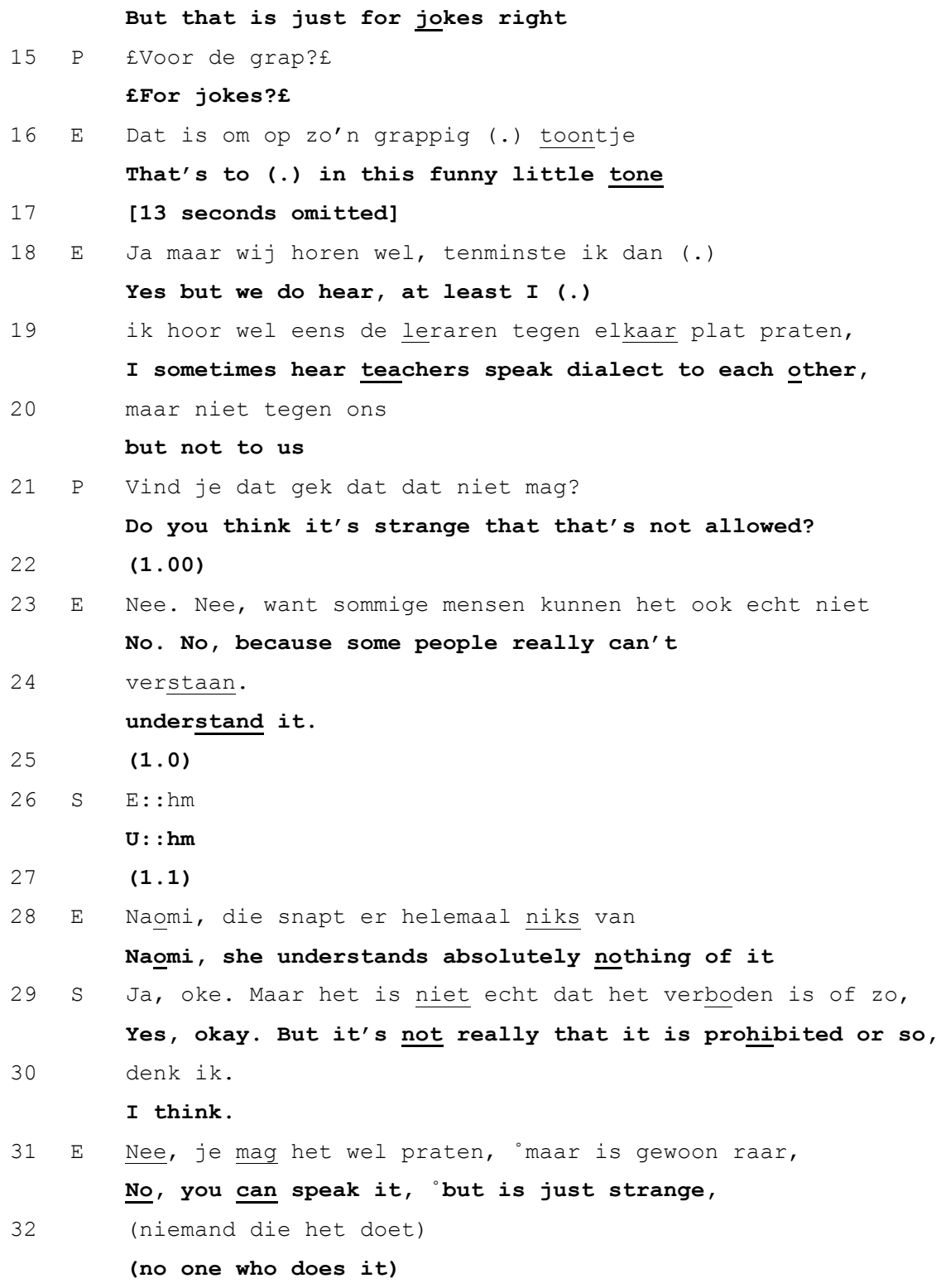

It is clear from this interaction that Sanne and Eva are not entirely certain of what is allowed when it comes to speaking dialect. At first, they agree that they cannot use it with teachers (lines 5-6), but they can with each other (line 6). Eva - who generally, throughout my fieldwork, seemed to feel more strongly than her friend that some contexts were inappropriate to speak dialect - mentions it would be 'weird' to speak Limburgish to a teacher. Her friend 
Sanne - who appeared less bothered by ideas about contextual appropriateness of Limburgish - notes that she has used it with one specific teacher. Considering the reactions of teachers to dialect use in class, which were not straightforwardly accepting but not condemning either (as in Example 6.5), it is not surprising that these pupils are not entirely certain of the policy.

After some discussion, Sanne and Eva more or less come to a consensus in lines 29-32 that speaking Limburgish at school is not prohibited, but not really appropriate (or raar 'weird'), especially with teachers. They have thus internalized the idea that Limburgish is the variety for informal, intimate interactions in the diglossic context of Limburg, and that it therefore logically does not occupy a place in formal school business or in hierarchical relations such as that between pupil and teacher, even if there may be exceptions (line 13). They relate this prohibition to an argument of understanding: they cannot speak dialect at school because "some people cannot understand it" (lines 2324). Applying the same reasoning a few minutes later, when I asked them about the prohibition against Turkish, they agreed that it was more logical that that was not allowed because "at least dialect is more understandable, at least it's Dutch."

This last comment reveals an ideology that was also recognizable in teachers' discourse about the position of different languages in the school. In this ideology, languages were hierarchically organized according to their degree of appropriateness in school. Standard Dutch was at the top of the hierarchy as the official medium of instruction and communication and was appropriate in all contexts. Limburgish followed: it was not really allowed, but not really prohibited either, as Sanne and Eva discuss in Example 6.6. At the bottom of the hierarchy were the languages that have their origins outside the Netherlands. Sanne and Eva build this hierarchy on the idea of comprehension: everyone understands Dutch, most people understand dialect (notice how, in line 26, Sanne seems to display disagreement with Eva's comment on some people not being able to understand dialect), and fewest people understand Turkish, Moroccan Arabic or Berber. The same hierarchy emerged from a conversation I had with a teacher and the school principal, described in Example 6.7. The principal builds his argument on something other than the argument of comprehension, however.

Example 6.7. Field notes, 20 March 2017.

During the break in the teachers' lounge, I ask the school principal and a teacher whether there is a policy on speaking dialect in class, and they immediately reply that it is not allowed. On the other side of me, there's another teacher who joins in the conversation and says that it does strike him that pupils often speak dialect among each other. 


\section{Nederlanders and buitenlanders}

The first teacher then starts talking about other languages that are not allowed. He tells us how he recently made a pupil (Hatice) leave the classroom because she spoke Turkish three times, and that that really is not allowed. The principal says something about how there is a more 'positive' way to stop pupils from speaking Turkish, for example by saying "if you want me to help you, you have to speak Dutch." Then he says: "But yes, that is definitely not allowed. And I know I am on thin ice here, but we're in the Netherlands here, so it really is different to speak Turkish than it is to speak dialect in class."

Like Sanne and Eva in Example 6.6, the principal and teacher in Example 6.7 have an immediate answer to my question about dialect, namely that it is not allowed. Interestingly, whereas Sanne and Eva are under the impression that they are allowed to speak dialect among each other, the teachers state that they are not. At the same time, another teacher notes that pupils often do so - it is thus clear, also from their side, that this rule is not reinforced very strictly. This reflects the position of Limburgish as appropriate in informal, intimate contexts.

The linguistic hierarchy outlined previously, in which Dutch is always appropriate, and then Limburgish, and lastly, Turkish, Berber and Moroccan Arabic, also emerges from this example. Rather than building on the argument of comprehension, however, here it is predicated on an ideology of appropriateness which builds on normalized links between language and place (Auer 2013; Quist 2010), according to which some languages can appropriately be spoken in some places, and other languages cannot. The principal's comment 'we are in the Netherlands here' constructs dialects as more acceptable than for example Turkish, because despite their official inappropriateness in the smaller context of the school, dialects are still appropriate in the larger place in which they are spoken, that is, in the principal's words, in the Netherlands. Turkish is not Dutch and is therefore deemed inappropriate in school, but furthermore, by being constructed as not 'from here,' it is implied to be inappropriate in the larger Dutch context. It is thus constructed as 'doubly inappropriate.' This evokes concerns about language choice as indexical of national belonging or loyalty. The phrase "I know I am on thin ice here" is rhetorically potent as a supporting strategy for his argument: it suggests that he has considered all sides of the dilemma and has come to the only logical conclusion (Liddicoat et al. 1994). It also points to the principal's awareness that there may be controversy around this issue. At the same time, he acknowledges that bluntly prohibiting the language would not be conducive to a positive relationship with the pupil, and suggests a somewhat 'softened' tone to convey the same message (Jaspers and Rosiers 2019). 
The logic of language appropriateness constructed around an ideology of language and place was often put in practice when pupils spoke Turkish in class. This happens in Example 6.8. I analyzed a shorter version of this fieldnote in Example 5.2 on page 140.

Example 6.8. Field notes, 19 December 2017.

Care and Well-being class. I'm sitting with Hatice, Amira and Dounia who are talking and laughing. Ms. Smit comes by and scolds them, telling them to concentrate on their work. This exchange follows:

Hatice: "Always those foreigners, isn't it..." ("Altijd die buitenlanders, hè").

Ms. Smit, rather forceful: "You're not foreigners, you're Dutch!" (Jullie zijn geen buitenlanders, jullie zijn Nederlanders!").

Hatice again: "But my culture is Turkish, Turkey number one!" Then she says something in Turkish and Ms. Smit tells her to speak Dutch. Hatice and the other girls protest and say "we live in a free country!"

Ms. Smit: "Yes, in the Netherlands, so speak Dutch, or you can go to another school."

In this field note the teacher scolds a number of pupils who are having a conversation in Turkish. Hatice's first reaction ('always those foreigners') is ironic. Hatice and the other girls around her commonly referred to themselves as 'foreigners' (buitenlanders), and here Hatice pretends to voice Ms. Smit's thoughts, negatively assessing buitenlanders in a turn of phrase that is recognizably reminiscent of Dutch racist discourse. When Hatice and the others repeatedly challenge what Ms. Smit says, and posit that they 'live in a free country,' Ms. Smit implies, in rather straightforward terms, that Dutch is the only language appropriately spoken in the Netherlands. As such, she constructs and reinforces the "deep-rooted conviction that languages, speakers and places constitute a unity" (Quist 2010, 632).

Policing did not only occur when pupils engaged in full conversations in, for example, Turkish or Arabic. As mentioned in the previous section, many pupils with and without migration background used elements of languages other than Dutch in their daily speech. They generally did this in conversations with each other, and not with teachers, and (like dialect) it was thus associated with informality and intimacy. However, I once witnessed Hatice (the same pupil as in the previous example) use an expression that pupils often used with each other in interaction with Ms. Smit (who also appeared in the previous example). The word she uses, "yallah," is associated with Arabic and can be translated as "come on" or "hurry up." The interaction occurred while Ms. Smit was practicing an exercise with Hatice, where Hatice had to name the steps to take when someone else is choking. Generally, the interaction was lighthearted and friendly, but Ms. Smit had also already 
corrected Hatice for her tone, which she said was 'too loud and agitated. ${ }^{33}$ She had thus already been monitoring how Hatice speaks, on top of checking whether she knew the steps of the exercise.

\section{Example 6.9. 16 May 2017. Participants: Hatice (H), Ms. Smit (S). Care and Well-being. Expression associated with Arabic is in italics.}

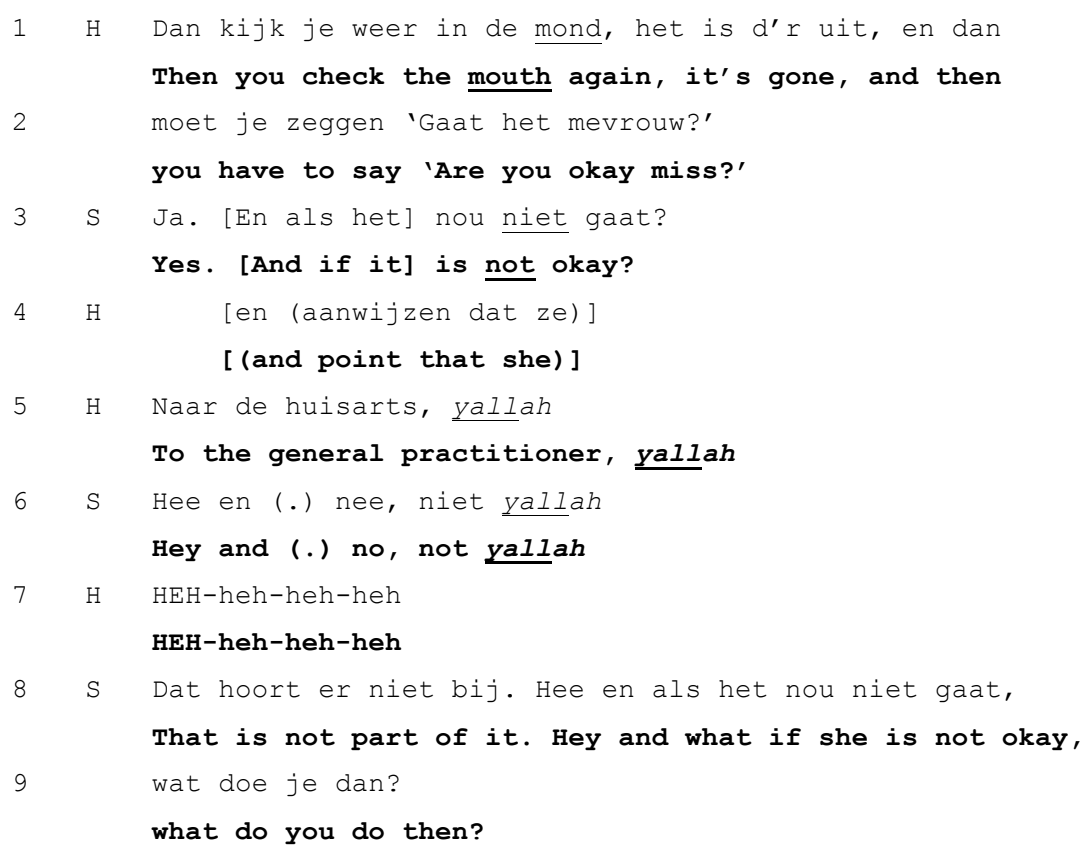

Ms. Smit's policing of Hatice's expression 'yallah' may have been motivated by her perception that this is colloquial language use and is unsuitable in the context they are discussing, that is, when performing a first aid intervention in an emergency situation. On the other hand, considering Ms. Smit's attitude toward pupils speaking Turkish (as seen, for instance, in Example 6.8), there is a fair possibility that she is reacting to this pupil's use of an expression associated with Arabic, and more so, to the fact that Hatice does this in direct conversation with her (that is, without masking it). Ms. Smit does not accept or reject the content of the answer Hatice has given but makes a point of telling Hatice that 'yallah' is not part of the sequence and repeats her question. Hereby, she shows that these kinds of exchanges are to be held in what she perceives as 'pure' standard Dutch.

${ }^{33}$ The perception of youth with migration background 'shouting' instead of speaking is also based on a language ideology, see Cornips, Jaspers, and de Rooij (2014). 
The examples in this section have illustrated the language policy as it was perceived by pupils (Example 6.6) and as it was put in practice by teachers (examples 6.5, 6.7, 6.8, and 6.9). Although the two examples of policing-inaction (Example 6.8 and 6.9) come from the same teacher, I also observed instances of policing by other teachers. For example, a teacher once told me that she punished a pupil who kept speaking Arabic despite being told not to. The teacher told the pupil that "if she wanted to speak Arabic so badly, she can go and translate the whole chapter into Arabic for me." The pupil had protested and said that she would not be able to, but in the end, she had produced a translation - which, by the way, the teacher herself could not correct (Field notes, 10 October 2017).

Language policing constructed and reinforced an ideology in which some languages - i.e. Limburgish - were officially not allowed in class but were perceived as appropriate to the larger geographical area in which they were spoken, and the prohibition was therefore not strictly reinforced and even unclear to some pupils. Limburgish was tolerated when spoken between pupils and sometimes between teachers and pupils. Other languages - that is Moroccan Arabic, Berber, Turkish - were strictly forbidden. They were policed when spoken in full conversation, but also as mere words inserted into utterances, between pupils and teachers as well as among only pupils. This prohibition was constructed as obvious, either based on an argument of comprehension ('other people do not understand') or appropriateness ('we're in the Netherlands'). ${ }^{34}$

Regarding the position of Limburgish, it could be noted that the acceptance of the use of dialect in this school illustrates a positive

\footnotetext{
${ }^{34}$ Interestingly, I never heard the argument of having to improve their proficiency in standard Dutch brought up as a reason, even though teachers did not seem to think highly of their pupils' standard Dutch. The absence of this argumentation is salient with a view to studies that demonstrate the dominance of views that relate pupils' migration backgrounds to (perceived) lower Dutch language proficiency (e.g. Bezemer 2007), relate lower language proficiency to lower educational attainment (Herweijer 2008; Huijnk and Andriessen 2016), in turn resulting in education policy that "fosters mainstream (language) education as the means for their social integration and emancipation (Bezemer 2003)" (Spotti and Kroon 2009, 179; also cf. Jaspers 2015). In the Netherlands, the link between migration background, language proficiency, and educational attainment seems to be made particularly for children in primary education, however. In secondary education the dominant view seems to be that socioeconomic position and parental education levels are more relevant than ethnic background for determining language proficiency and educational attainment (Bezcioglu-Göktolga and Yagmur 2018; Herweijer 2008; van Der Slik, Driessen, and de Bot 2006).
} 
development. Ideologies of dialects being associated with educational disadvantage seem to have lost ground, at least at this school, and I did not observe instances of strict prohibition. On the other hand, in South High School dialects were still associated only with informal side-activities and intimate conversations rather than more prestigious contexts of use, and furthermore, they were treated as exclusive to people whose (grand)parents had been born and raised in Limburg, whereas pupils with migration backgrounds did not use them even if they might have had competence (cf. Cornips 2020a). Furthermore, the acceptance of dialects seemed to exist especially when contrasted with languages like Turkish or Arabic. The idea that languages belong to speakers, who belong to places (Quist 2010) made dialects acceptable since they were "indexical of local belonging" (Cornips and de Rooij 2015, 91); whereas Turkish, Moroccan Arabic or Berber often seemed to be perceived as indexical of local unbelonging (Thissen 2018). This made the former languages into a normal and acceptable occurrence, and the latter into a problem.

However, the pupils of class $3 / 4 \mathrm{~b}$ were not merely passive recipients of language policies and policing (cf. Jaspers 2005a, 2011b), nor were teachers their passive enforcers (cf. Jaspers 2014). As I describe in the following section, I regularly heard pupils challenging, disobeying, but also imitating language policing. Teachers also playfully engaged with or broke the policy.

\subsubsection{Dealing with policing: Challenges, mockery, and imitation}

Pupils whose languages were the subject of policing noticed the discrepancies and contradictions in the reinforcement of the language policy. They found it unfair that they were not allowed to speak Turkish, for example, while others were not disciplined when using Limburgish. They also regularly expressed this to teachers, as in the example below.

\section{Example 6.10. 18 April 2017. Participants: Ms. Smit (S), Meryem (M).}

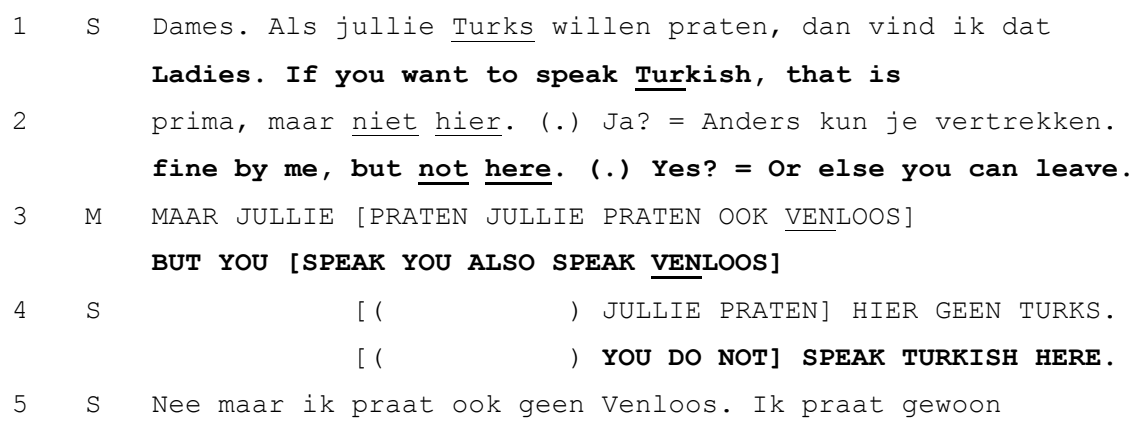




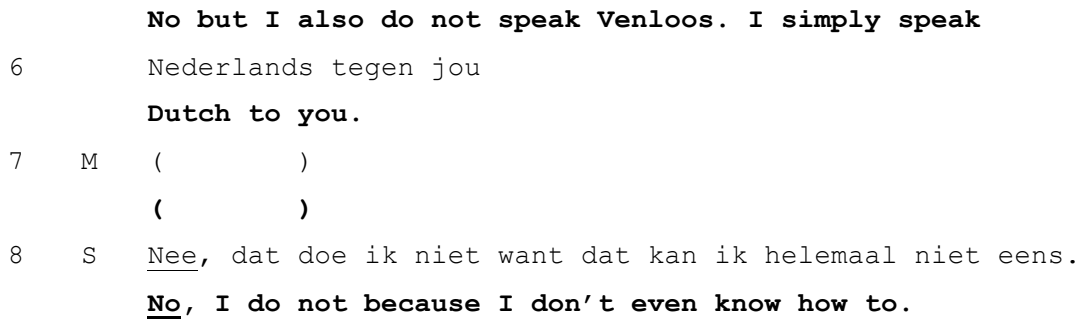

Right before this exchange, Meryem was speaking Turkish with Yildiz. When Ms. Smit reprimands them, Meryem retorts by saying that 'you' (plural) also speak Venloos (line 3). This 'you' can be heard to refer to teachers or to dialectspeakers. In a way, Meryem is right: Many teachers often spoke dialect together, and at times even with pupils, and the use of dialect also usually went unpunished. Ms. Smit did not speak dialect, however. As she says in line 6, she "doesn't even know how to." Hereby, she denies her own individual use of dialect, but does not deny it of the group of which Meryem made her a part. Such responses to language prohibitions were commonplace among these pupils (for example, I also heard it occur between Ms. Bos, Meryem and Yildiz, Field notes, 24 March 2017). They often pointed to the unfairness of not being allowed to speak Turkish, but pupils also imitated the linguistic policing they were subjected to by teachers with each other. Take for instance Meryem, who previously in Example 6.10 was upset about not being allowed to speak Turkish.

Example 6.11. Field notes, 15 May 2017.

Math class. Ali is repeatedly yelling a word; I can't really hear what it is. Seeming slightly irritated, Meryem asks Amira next to her what he is saying. "He is just saying look'," replies Amira. "That's Berber." - "We are in the Netherlands!" ["We zijn in Nederland!"] Meryem says, in a loud, but subtly smiley voice. She has probably heard this plenty of times herself, since she is often corrected for speaking Turkish.

The way in which Meryem comments on Ali's statements in Berber is very similar to how teachers policed utterances in languages other than Dutch. She does not say "you may not speak Berber"; instead, the mere mention of the geographical area in which the utterance takes place makes it recognizable as an admonition of the kind teachers often produced. It constructs the use of Berber as inappropriate and thus builds on the language ideology illustrated previously. It shows how Meryem has internalized the policy and the reasoning behind it (appropriateness of languages in certain places), and how 
she instrumentalizes it when she does not understand, or wants to silence or provoke another pupil.

In Example 6.11, Meryem imitated teachers' prohibitions by using a tone that suggested irony, but pupils also sometimes ridiculed prohibitions in a more obvious jocular way, for example by telling each other 'Je mag geen buitenlands praten!' ('You are not allowed to speak foreignish!'; field notes 15 May 2017) or 'Niet moslims praten, alsjeblieft' ('Don't speak Muslimish, please'; field notes 23 January 2018). These examples - which the pupils said they had heard in real life - attest to their awareness of stigmatization of a conflation of languages, associated speakers, and religion ('Muslimish'), that are constructed as 'not belonging' in the Netherlands (Duyvendak 2011), and an accompanying disinterest for the specific language that is actually being spoken ('foreignish').

Although (or maybe because) teachers did not usually prohibit the use of Limburgish, pupils who were themselves regular subjects of linguistic policing also policed other pupils' utterances in Limburgish. This usually occurred in a playful fashion, but it nonetheless suggested that they felt that teachers' enforcement of the language policy was not entirely equal.

\section{Example 6.12. Field notes, 14 February 2017.}

I am sitting at a table where Amira, Meryem, Emma and Nikki are doing assignments. A couple times, I hear Emma and Nikki speak Limburgish together. Amira is quick to 'correct' them (though playfully): "We don't speak Tegels here!"

In this example, we again see the association between place and language, in which place ('here') acts as a justification for prohibiting a language. These examples of pupils policing each other not only show that the pupils are aware of (and play with) the restrictive language policy, but also that they are willing to use it with each other in "acts of social policing" (Møller 2019, 40; see also Evaldsson and Cekaite 2010).

Pupils without migration background did not usually engage in linguistic policing - not of Limburgish, nor of Turkish, Moroccan Arabic or Berber. The example below, in which Nikki asks a teacher intern for a translation of an utterance in Berber, was a rare exception.

\section{Example 6.13. Field notes, 29 March 2017}

Care and Well-being class. I overhear Mounira - a teacher intern - say something to Dounia in what I am guessing is Berber. Nikki, who is standing nearby, says: "Hey, I wanna understand that too!" Mounira: "I said 'Just go ask the other teacher that, dear." Nikki sounds playful: "If you say something in Moroccan, you should translate it, then I can join the conversation." Mounira then asks Nikki if she is from 
Tegelen. When Nikki replies she is from Venlo, Mounira casually repeats the sentence she has just translated from Berber into (what sounds to me as) Venloos.

Mounira, the intern, had a Moroccan background, had grown up in Venlo, and was the only person with a migration background that taught class $3 / 4 \mathrm{~b}$ in the time that I spent with them (as an assistant teacher, for a few months). The scene in Example 6.13 deviates from what was common at South High School in a number of ways. As mentioned, pupils without a migration background - like Nikki - would not usually comment on uses of languages other than Dutch or dialect. In this instance, Nikki does do so. She does not just ask for a translation, but comments on the practice of using another language, invoking the argument for linguistic policing that states that 'everyone should be able to understand.' Mounira translates what she has just said, and Nikki makes another more generalized comment about using 'Moroccan,' suggesting that her intervention was not merely about this particular utterance, but about the practice of using 'other' languages. Although she provides an immediate and 'innocent' reason for it (wanting to “join the conversation"), Nikki's request to translate a 'Moroccan' utterance can be understood as a reprimand to Mounira. Strictly speaking, Mounira was disobeying the school's language policy by speaking Berber with Dounia. In reply to Nikki's comment, she again casually infringes on the policy by using the same approach with Nikki as she did with Dounia - that is, by speaking to her in what she seems to assume is Nikki's 'own' language (Venloos). Hereby, she shows her linguistic repertoire to span wider than Dutch and Berber only. To the extent that Nikki's reprimand could be interpreted as a form of 'othering,' she has countered it by using a variety that indexes being 'from here.'

It happened more often that Mounira broke the standard Dutch language policy. I once heard her switch from standard Dutch into dialect mid-sentence when privately giving Sanne and Eva instructions during class (Field notes, 21 June 2017). Although such private use of Limburgish is common and often considered appropriate (e.g. Morillo Morales 2017), these pupils appeared confused by it and replied in standard Dutch, after which Mounira also continued in standard Dutch. In marked contrast to the pupils with migration backgrounds, who associated Limburgish exclusively with people without migration backgrounds, or with stylizations, Mounira thus seemed to have no reservations to display competence in dialect without a humorous or sarcastic undertone - even if that use was not always ratified by her interlocutors.

These examples show that many teachers can be seen to play with language policies, or implement it only partially or sometimes, even if they 
might agree with that policy in theory (Jaspers 2015). I also observed this attitude with Mr. de Jong, a young math teacher. Mr. de Jong showed aboveaverage interest in his pupils in several ways, for example by engaging in conversation about religion with them during class, or by asking them about the music they liked. Mr. de Jong also used language to connect with his pupils, employing words or phrases associated with Arabic or Turkish. As he explained to me: "I do that on purpose, to confuse them, or attract attention, or to break expectations" (Field notes, 19 June 2017). In Example 6.14, it certainly attracts the pupils' attention. This interaction occurred during math class, while Khadija was correcting her assignments with a corrections booklet. Mr. de Jong came by her desk to check how she was doing. I was sitting behind them and did not participate in the interaction.

Example 6.14. 15 May 2017. Participants: Mr. de Jong (J), Khadija (K), Amira (A). math class.

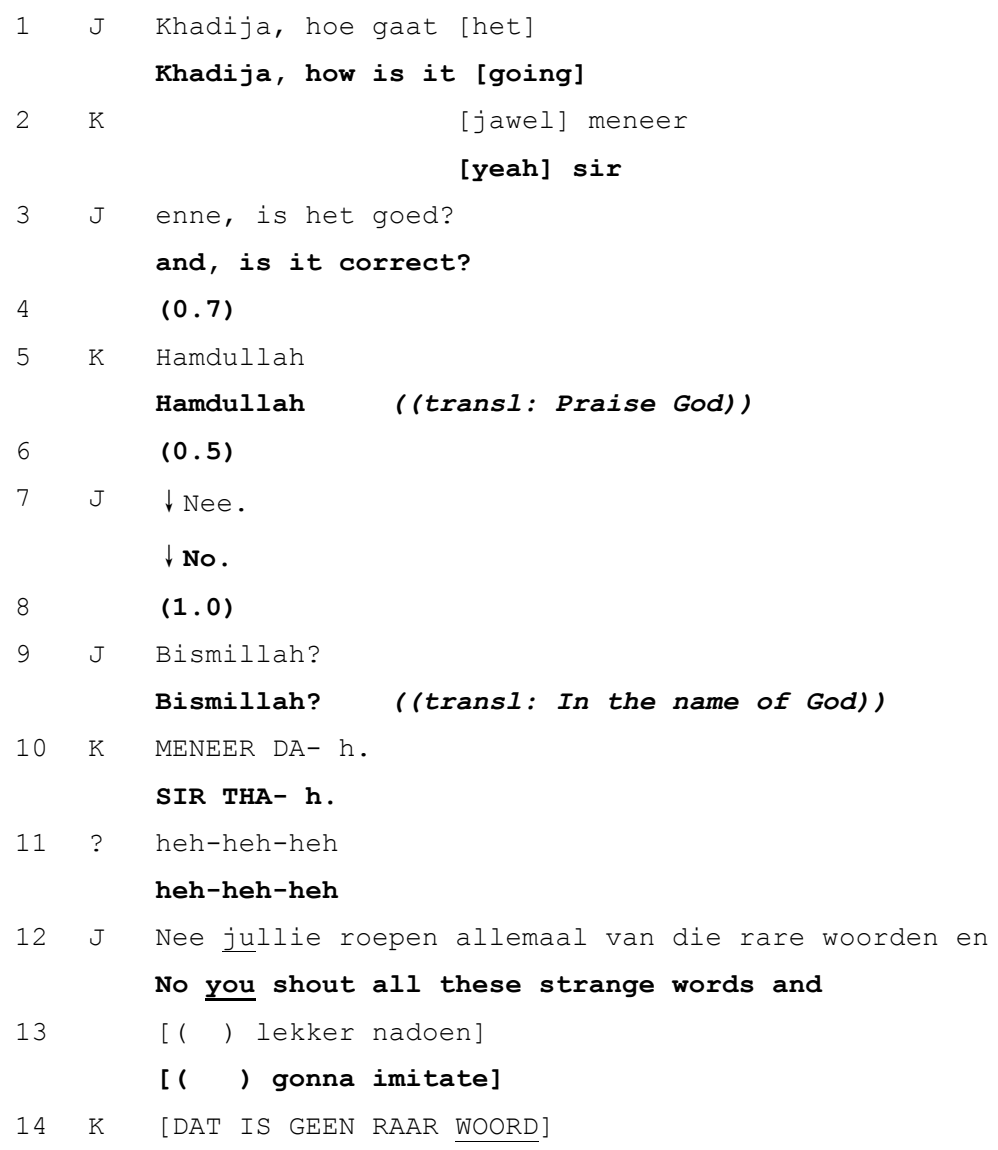


[THAT'S NOT A STRANGE WORD]

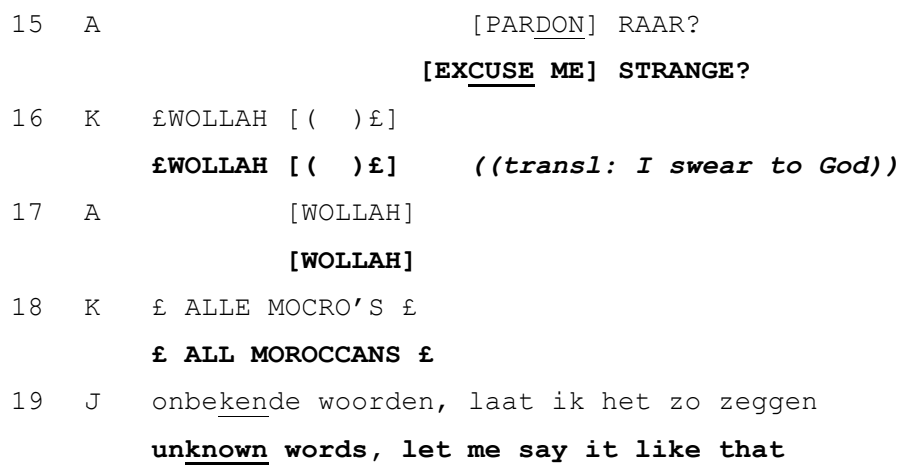

In line 5, Khadija replies to Mr. de Jong's question using an expression associated with Arabic: Hamdullah (translatable as 'Praise God'). This could be interpreted as a cheeky way to answer, even if it was produced in a lighthearted tone. Khadija addresses Mr. de Jong in a code that she knows he does not share: in the pupils' categorization scheme, Mr. de Jong was a Nederlander ('Dutch person'), without migration background, who was not Muslim and could not be expected to know Arabic (even if 'hamdullah' is a rather common expression). Saying "hamdullah" directly to him could be seen as willfully positioning him as an outsider, since he might not understand exactly what she is saying to him. But primarily, Khadija infringes on the language policy while addressing the teacher.

Indeed, the short gap in line 6 indicates some interactional trouble, and in line 7, Mr. de Jong produces a short disaffiliative response. "No" in line 7 indicates trouble in reaction to the previous utterance "hamdullah," but the teacher does not specify why this is trouble. It seems likely that he rejects it because it is Arabic, since the meaning of it ('praise God') - if he knows that - hardly seems ground to reject it. However, in the pause in line 8, he seems to have changed his mind because he then utters an expression ("bismillah") that pupils also sometimes used and that is similar to Khadija's turn in that it is associated with Arabic and it calls on God. With his "Bismillah," he appears to step down from his initial rejection of Khadija's utterance. Khadija's reaction in line 10 is loud and suggests rejection of Mr. de Jong's utterance maybe because it is not 'his' code, or maybe she does not approve of the way he uses it. These reactions become stronger when he says that "they shout strange words" (line 12), to which Amira and some other pupils join the interaction in challenging this negative assessment. Despite these apparently disaffiliative turns, the interaction maintains its lighthearted, humorous undertone, judging from Khadija and Amira's smile voices in lines 16-18. Mr. 
de Jong seems to have sufficient rapport with the pupils to get away with what pupils might have perceived as unforgivable transgressions in some other teachers.

Mr. de Jong was more linguistically creative than most teachers of class $3 / 4 \mathrm{~b}$. Like the teacher that Jaspers $(2014,387)$ describes, he regularly "insert[ed] indexically charged linguistic materials that reversed linguistic expectations." At the same time, he could be observed to invest in pupils' 'proper' use of Standard Dutch, correcting them if they uttered non-standard (but common) phrases such as “beter als jou” ('better than you,' Field notes 19 June 2017) or "welke papier" ('which paper,' Field notes 8 May 2017). Rather than seeing this as a contradiction, Jaspers (2015) argues that such behavior can be understood as a logical consequence of teachers' need to reconcile various goals, that is, to implement the language policy while also maintaining a positive classroom climate. This seems to have been the case here.

\subsection{Conclusion}

This chapter has analyzed multilingual behavior at South High School; the frictions this caused with the monolingual language policy; how teachers policed the use of different languages differently and thereby constructed and reinforced a linguistic and social hierarchy; and how teachers as well as pupils negotiated language policies in practice. As such, the aim was to examine the relations between categorization practices and the use (and policing) of different linguistic resources in South High School.

South High School did not have an explicit language policy, but it was assumed and taken for granted that everyone at school knew that they should speak standard Dutch. Pupils used a variety of other linguistic resources in their daily interactions at school, however, including Limburgish, Moroccan Arabic, Berber, and Turkish. Use of those languages was distributed in a particular way: only (some) pupils without migration backgrounds spoke Limburgish. Pupils with migration backgrounds very rarely spoke Limburgish and only in stylizations, even if they had (passive or active) knowledge of Limburgish. Pupils with Turkish migration backgrounds sometimes spoke Turkish with each other. All pupils - mostly those with migration backgrounds, but also some without - often used words or phrases from Moroccan Arabic, Berber, and sometimes Turkish in their otherwise Dutch utterances.

The indexicalities of those linguistic resources differed. The use of expressions associated with Moroccan Arabic, Turkish and Berber seemed to enable pupils to claim a particular position in the local social hierarchy: it bestowed a form of social prestige on the speaker. Limburgish did not have 
this same effect. Although many pupils used it in their interactions, it was not associated with a 'cool' persona the way Arabic expressions, for example, seemed to be in the eyes of the pupils. The symbolic organization of languages among pupils thus reflected patterns of local social stratification as explored in the previous chapters (cf. Nørreby and Madsen 2018), whereby to the pupils, the categories buitenlander, Turk and Marokkaan were associated with local prestige, and the category Nederlander was treated as less prestigious.

The reactions of teachers to those languages showed quite an opposite organization. Teachers applied the monolingual Dutch language policy differently: They enforced the standard Dutch language policy at school particularly when pupils spoke Moroccan Arabic, Berber and Turkish among themselves, and not when they spoke Limburgish. They displayed a "deeprooted conviction" (Quist 2010, 632) that links languages and their speakers to places. Several teachers as well as the school principal built on ideologies according to which certain languages were appropriate because of the place in which they were spoken, positing that because pupils were in the Netherlands, they had to speak Dutch (Example 6.7 and 6.8). As such, speaking Turkish, for instance, was framed as not related to the Netherlands and therefore inappropriate. When compared to other accounts of language policing in Dutch classrooms (e.g. Bezemer 2003; Bezemer and Kroon 2006; Spotti and Kroon 2009), the reinforcement was remarkably rarely justified by a supposed need for pupils to improve their standard Dutch - even though many teachers were quite vocal about their view of pupils' Dutch as less than perfect. Instead, the argument was usually that of geographical appropriateness or the need for mutual understanding.

Teachers' marked insistence on standard Dutch with pupils who used Turkish, Arabic or Berber, and the lack of reinforcement with dialect-users, invokes shifted and shifting concerns surrounding languages and their associated speakers in Dutch society. The use of dialects by school-going children used to be a major source of concern for educators until around the 1970s, as they were associated with 'uncivilized' speakers and educational disadvantage. This has gradually shifted: even though dialects remain relegated to the informal sphere and generally lack a ratified position in schools, they are no longer as stigmatized as they once were. Concerns about language as indicative of 'civilization' have shifted to concerns about language and belonging. In Chapter 5, this could be recognized in teachers' reactions to pupils' self-categorizations, and in the current chapter, in their policing of languages. It furthermore demonstrates how the symbolic organization of languages reflects and reproduces ever-changing societal concerns and patterns of social stratification (cf. Nørreby and Madsen 2018). 
National languages can thus come to "stand for concerns as different as national unity, effective communication, social mobility, and civic duty" (Jaspers 2017, 706). As Mosher $(2015,250)$ argues, "[s]peaking Dutch in the public sphere is understood by many 'native' Dutch as an expression of commitment to Dutch society on the part of non-Western Dutch citizens and newcomers." In South High School, speaking Dutch seemed to be enforced not necessarily out of a concern for pupils' language skills, but rather as a matter of principle: The 'good pupil' uses standard Dutch. The pupil who uses Limburgish may not be that same kind of good pupil, but this language choice at least indexes 'belonging' in Limburg (and thus the Netherlands) rather than in another country. Conversely, speaking Turkish, Arabic or Berber can come to be seen as an expression of a lack of such commitment. This suggests that language policing was a site for discussing ideologies of belonging. 


\section{Conclusion}

The aim of this dissertation has been to analyze how pupils of a secondary school in Venlo, the Netherlands, negotiated local as well as wider societal social hierarchies through ethnic categorization and the use of different linguistic resources. The research question introduced in Chapter 1 was: What do ethnic categories and linguistic resources mean, and do, for the pupils and teachers of South High School? I have examined this on the basis of data gathered through nine months of ethnographic fieldwork with pupils of a secondary school class ('class 3/4b' of 'South High School') in Venlo, the Netherlands, and by analyzing interactions between pupils, teachers, and myself, mainly with tools from Membership Categorization Analysis (MCA) and Conversation Analysis (CA).

About half of the pupils of class $3 / 4 b$ had a migration background, and although they were born in the Netherlands, they regularly categorized themselves and each other with the labels buitenlander 'foreigner,' Marokkaan 'Moroccan,' and Turk 'Turk,' and referred to others without a migration background as Nederlander 'Dutch.' In much public discourse in the Netherlands, as well as in some academic research, such categorizations are interpreted as offering an insight into the 'integration' or 'feelings of belonging' of immigrants and their descendants. I have argued that such interpretations offer, at best, a partial explanation of the phenomenon of ethnic categorization, as categories may not mean the same for everyone and in every context. Furthermore, categorizations can be understood not only as providing information about the categorizer's sense of self, but they are also actions in interaction. To understand what ethnic categories mean, and what people might gain from using them, I took a sociolinguistic-ethnographic approach. I went to school with the pupils of class $3 / 4 \mathrm{~b}$ for nine months, conducting participant observation, taking field notes and making audiorecordings of interactions at school. On the basis of close analyses of interactions on the local level, I demonstrated i) that widespread national/ethnic categories had various local meanings, ii) that categorizations and the use of linguistic resources contributed to the emergence, maintenance, and negotiation, of local social hierarchies, iii) that categorizations were in addition tied to interactional necessities, and iv) that pupils and teachers not only negotiated local social hierarchies, but that they also engaged with widerspread definitions and hierarchizations of categories and linguistic resources. 
In this conclusion, I will first summarize the main findings, to then discuss the theoretical relevance and implications of those findings. Then, in Section 7.3, I will comment this study's representativity, and I make suggestions for further research.

\subsection{Summary of main findings}

In Chapter 3, I analyzed the complex meaning potential of the categories Marokkaan, Turk, Nederlander and buitenlander among the pupils of class 3/4b. These categories carry a history of meanings with them, which was negotiated, contested, reinforced and adapted in this context of use. In class $3 / 4 \mathrm{~b}$ of South High School, the idea of descent was the main determinant of a pupil's categorization, and it was treated as common-sense knowledge that everyone was member of one of those categories. The implications of membership of a category such as Turk or Marokkaan were constantly negotiated, however. Pupils treated factors such as religion, physical appearance, style, or behavior as somehow related to category membership, but the ways in which those relations found expression and how essential or central they were to category membership were often negotiated. Furthermore, pupils often recognized exceptions to what they presented as set characteristics of categories: the appearance of some people did not conform to what their assigned category was said to look like, or some people did not behave in the ways described by the pupils as indicative of their category. This did not change their 'knowledge' about that category, however. Based on these findings, I argued that the use of the labels Marokkaan, Turk or buitenlander does not necessarily indicate a lack of belonging or emotional attachment to the Netherlands, nor the presence of such attachment to another country, as has been implied previously (e.g. Azghari, Hooghiemstra, and van de Vijver 2015; Verkuyten and Yildiz 2007). In this chapter, I furthermore showed how the categories that are often stigmatized in Dutch society carried prestige among the pupils of South High School. It was 'cool' to be Marokkaan, Turk or buitenlander, and the category Nederlander was mainly associated with behavior that was locally unprestigious, such as being obedient. I have argued that this reversal of the wider societal hierarchy of prestige of these categories (in which Nederlander is more prestigious) can be seen as a strategy for these pupils to deal with, and challenge, their marginalized position in Dutch society. Their migration background, as well as their position in the lower echelons of the Dutch educational system, meant that they grappled with membership in at least two stigmatized categories. Having changed the indexicalities of one of the categories by which they are defined by the outside world, they were able to enjoy a power and prestige among their peers at school that they were not 
often accorded outside that context (and which, in fact, was not shared by everyone at the school, as seen in Chapter 5 about the role of teachers).

In Chapter 4, I applied conversation analytic tools to examine how references to labels functioned in the organization of interaction and what pupils achieved by referring to categories. By mentioning categories, the pupils evoked a wide array of associations (e.g. 'members of different categories have different customs'; 'the category Marokkaan is discriminated against,' etc.). Analyzing interactions with references to categories on a turn-by-turn basis, I demonstrated that by mentioning categories, pupils evoked those associations, which in turn enabled them to make interactional moves. In that sense, the labels were "[useful] in getting things done" (Whitehead 2012, 1261): they enabled pupils to achieve moves such as shifting power positions in interaction and engaging in jocular mockery. Humor with categories was a particularly prominent interactional practice among the pupils of $3 / 4 \mathrm{~b}$. Pupils with a migration background often insulted the category of which they themselves, or their conversational partner, were seen to be a member, in ways that resembled societal discourses of xenophobia. Such moves of vari-directional double voicing often appeared to mitigate the effects of stigmatizing and hurtful discourses and allowed pupils to comment on structures of inequality and marginalization that disadvantaged them. Not just any pupil could make those moves by using the categories Turk, Marokkaan or buitenlander, however. It was difficult for pupils labelled Nederlander to engage in jocular mockery with a reference to one of those categories: they could be charged with racism since they were less easily understood as engaging in jocular mockery when engaging in banter of categories that were not considered 'theirs.' In those moments, wider societal associations with these categories surfaced, where categorization can imply othering and stigmatization. As such, the practice of categorizing others as Turk, Marokkaan or buitenlander was not a 'free for all.' Pupils who self-categorized as Turk, Marokkaan or buitenlander could engage in interactional work by referring to those categories in interaction, but pupils labelled as Nederlander could not do so without being policed. Finally, in this chapter I noted that one consequence of this frequent categorization is that it constitutes a mechanism by which ethnic categories are constantly reproduced. Their usefulness motivated pupils to keep using them; but this continued use at the same time reified the existence of those distinct categories of people (Whitehead 2012).

Chapter 5 reviewed the role of categorization practices in interactions involving teachers. This chapter showed that pupils were not the only 'categorizers' at South High School; teachers also formed part of the interactional processes by which categories were negotiated and endowed with meanings and functions. I demonstrated that teachers sometimes 
problematized pupils' self-categorizations as buitenlander, Turk, or Marokkaan in interaction with me and with pupils themselves. This appeared to stem from conventional interpretations of those labels that are wide-spread in Dutch society, such as that self-categorization as 'not-Dutch' indicates a lack of belonging in the Netherlands, or that using the categories buitenlander, Turk, or Marokkaan is stigmatizing. In some interactions, disagreeing with a pupils' self-categorization could also be understood as having a function in interaction, for instance to reinforce authority or discipline a pupil. While pupils shifted between, on the one hand, uses of category labels with more conventional associations, and on the other hand, they used categories with locally constructed associations (such as being 'cool,' or being relaxed about rules, as primarily analyzed in Chapters 3 and 4), teachers showed interpretations of those categories mostly in reference to their conventional associations. Aside from problematizing pupils' self-categorizations as buitenlander, Turk, or Marokkaan, however, teachers could also be seen to engage in discourses that positioned their pupils with and without migration backgrounds as categorically different. Teachers seemed to struggle with the apparent contradiction that they preferred their pupils with a migration background to self-categorize as 'Dutch,' but at the same time, they knew that there were differences between pupils with and without migration background which were furthermore emphasized by the pupils themselves. I ended the chapter by emphasizing it is not per definition bad or irresponsible for teachers to use the labels their pupils use. What I argued, instead, is that it seems unlikely that pupils will feel (more) included when their teachers problematize the labels 'Moroccan,' 'Turk,' or 'foreigner,' or urge their pupils to label themselves 'Dutch,' while they - and society at large - also position them as are categorically different from people without migration backgrounds. When teachers reject labels that pupils can hardly escape from, or that they have reappropriated, they might be seen to imply that being 'Dutch' is better. Stimulating a sense of inclusion for all pupils might be more effective when using categories that are not already subject to local renegotiation.

If the symbolic organization of categories as described in Chapters 3 to 5 was one of the ways in which pupils engaged in the negotiation of local and wider societal social hierarchies, Chapter 6 about multilingual behaviour at South High School described how the symbolic organization of linguistic resources was another one. Although the school did not have an explicit language policy, teachers took for granted that pupils knew that they had to speak standard Dutch in class. This caused frictions, as pupils had a variety of 
linguistic resources at their disposal, which aside from standard Dutch ${ }^{35}$ (which they used most frequently to communicate at school) also included Limburgish, Moroccan Arabic, Berber, and Turkish, and they used all those resources in their daily interactions at school. The indexicalities of those linguistic resources differed: among the pupils and in the context of school, the use of expressions associated with Moroccan Arabic, Turkish and Berber bestowed a form of social prestige on the speaker, and Limburgish did not have this same effect. The symbolic organization of languages among pupils thus reflected patterns of local social stratification as explored in Chapters 3 to 5 . The hierarchization of those languages by teachers, as suggested by their policing of languages other than Dutch, followed a reverse pattern. They enforced the standard Dutch language policy particularly when pupils spoke Moroccan Arabic, Berber and Turkish, and not when they spoke Limburgish, and justified this by building on ideologies of languages and their speakers as tied to certain places. Limburgish was acceptable due to the school's location in Limburg, while Turkish, for instance, was framed as not related to the Netherlands and therefore inappropriate. In the chapter, I suggested that teachers' marked insistence on standard Dutch with pupils who used Turkish, Arabic or Berber, and the lack of reinforcement with dialect-users, invokes shifted and shifting concerns surrounding languages and their associated speakers in Dutch society more generally. Whereas the use of dialects by school-going children used to be associated with 'uncivilized' speakers and educational disadvantage, today dialects did not appear to be as stigmatized as they once were in this context (even though they remain relegated to the informal sphere). Concerns about language as indicative of 'civilization' have shifted to concerns about language and belonging. This concern with belonging could be recognized in teachers' reactions to pupils' selfcategorizations, as well as in their policing of languages. This demonstrates how the symbolic organization of languages reflects and reproduces everchanging societal concerns and patterns of social stratification (cf. Nørreby and Madsen 2018).

\subsection{Contributions}

Here, I wish to underline four main contributions of this dissertation. By means of these contributions, I highlight and summarize how, throughout the dissertation, I have aimed to enter into dialogue with different bodies of

\footnotetext{
${ }^{35}$ In this context, 'standard Dutch' should be understood as Dutch as it is spoken in this particular area. This may not be perceived as 'standard' by speakers from other regions of the Netherlands.
} 
literature, including social scientific literature on ethnic identification, immigrant integration, identity, and belonging, and literature in the disciplines of sociolinguistics and anthropology generally.

\section{The meanings and functions of ethnic category labels cannot be taken for granted but} must be studied in context

This dissertation contributes to a deeper understanding of ethnic labelling practices by examining their situated meanings and interactional functions, while also taking into account their relations to larger scale processes and discourses. Migrants' descendants have been observed to label themselves in reference to their (family's) country of descent (rather than the country of residence) in countries with histories of immigration comparable to the Netherlands, such as Germany (Bozay 2012), France (Simon 2012), and Belgium (Jaspers 2011a). Although much sociolinguistic and anthropological work has emphasized that ethnic identities are context-specific, intersubjectively and discursively constructed, and negotiated in interaction (e.g. Androutsopoulos and Georgakopoulou 2003; Jaspers 2005b; Jørgensen 2005; Rampton 2005), this practice of self-labelling in terms of the country of descent is still often interpreted as signaling a lack of identification with, or orientation to, the country of residence (e.g. Agirdag, van Houtte, and van Avermaet 2011; Ersanilli and Koopmans 2010; Huijnk and Dagevos 2012; Phalet and Swyngedouw 2002; Verkuyten and Yildiz 2007). Research that asks people for the ethnic label they identify with insufficiently takes into account that the use of a label merely is an expression of identification with a label, which does not necessarily go hand in hand with, for instance, specific sociocultural orientations (Slootman 2016). Aside from conventional and wide-spread meanings, labels such as Turk, Marokkaan, buitenlander and Nederlander can take on different or additional meanings among individuals in local contexts. A key contribution of this dissertation is therefore to show that in order to understand what ethnic labels mean and what people might gain from using them, it is necessary to focus on interactional data in a context well known to the researcher, in other words, to take a sociolinguisticethnographic approach that takes into account local contingencies as well as relations to larger scale processes and discourses.

This approach has enabled me to demonstrate that the use of the labels Marokkaan, Turk or buitenlander does not necessarily indicate a lack of belonging or emotional attachment to the Netherlands, or the presence of such an attachment to another country. Participants of this study used these labels to construct local social personae and negotiate social hierarchies. Relatedly, this dissertation has underlined the importance of looking further 
than the concept of 'identity' when analyzing the practice of self- and othercategorization in ethnic terms. Even when 'ethnic' terms are used, 'ethnicity' or 'identity' may not be the main issue on participants' minds. Research participants in this dissertation could also be seen to pursue interactional moves when using references to ethnic labels, such as (dis)aligning with others, joking, or negotiating institutional power relations. By highlighting those interactional functions, I hope to have countered what has been referred to as a "fetishization of ethnicity" (Rampton 2005, 7), that is, the tendency to exoticize the Other and read 'ethnic identity' into ordinary actions such as joking or negotiating interactional power.

Combining ethnographic methods for data collection, and ethnomethodological approaches for data analysis, helps to avoid clichéd constructivism as well as groupism in studying ethnic identification

A key problem that has been identified in many studies on ethnic identity is that, on the one hand, researchers routinely claim an adherence to the idea that ethnicity is 'fluid' and 'constructed,' but all the while, often use groupist language in analyses, thereby portraying the world as consisting of bounded and self-explanatory 'ethnic groups' (Brubaker 2004). In this dissertation, I have aimed to avoid such groupism by taking ethnic categories as the object rather than the means of analysis to explain the persistent idea of ethnicity in participants' lives (Brubaker 2002). I have elucidated the merits of combining ethnography and the ethnomethodological approaches of Conversation Analysis (CA) and Membership Categorization Analysis (MCA) to do so. As such, the dissertation has contributed to the study of ethnic identification by using a particular combination of ethnographic methods to collect data, and Conversation Analysis and Membership Categorization Analysis to analyze that data.

One of the main goals of ethnography, as well as of CA and MCA, is to focus on the 'insider's perspective,' that is, to prioritize research participants' orientations to, and views of, a topic, rather than departing from the researcher's own goals or ideas. There are many ways in which these approaches differ, though, and in this dissertation, I hope to have shown how they can complement one another. On the one hand, the ethnographic foundations of this dissertation have strengthened the analyses of micro-scale interactions in many ways, the most important of which are, first, that most of the interactions I analyze in the dissertation have been selected as examples of common interactional patterns among the participants, and I analyze them to elucidate aspects of the social context. Without the long-term commitment of ethnographic fieldwork, I would not have been able to tell common and 
uncommon interactions apart and would not have been able to argue which interactional functions of referring to ethnic category labels were common among the research participants. Secondly, ethnography has helped me explore the situatedness of the interactions that I analyze, by acknowledging that all talk is embedded in historical, social, and cultural contexts (Moerman 1988). That is, I have analyzed micro-interactional patterns in light of those larger contexts, to see how those different scales interact with, and influence, one another. Finally, the emphasis on reflexivity in ethnography has helped me acknowledge and investigate my own role in shaping the context and the interactions that I analyze. On the other hand, the use of tools from CA and MCA has strengthened my ethnography in enabling me to explore the phenomenon of ethnic categorization in a systematic and thorough manner. By using the detailed transcription system of CA and MCA, I was able to examine the data that I collected and the arguments that I made with other researchers, and it was (and will be) easier for them to re-examine those data and contest my analyses than it is with field notes of one-time occurrences (Rampton et al. 2004). Principally, however, the merit of CA and MCA has been that they helped me to prioritize a focus on participants' concerns in interactions, enabling me to look beyond the 'ethnic' aspect of ethnic labels. In sum, it is the combination of these approaches that led, in this dissertation, to important insights into the functions and meanings of references to ethnic categories in conversation.

Manifestations and negotiations of 'diversity' as a result of globalization should (also) be studied in areas perceived as peripheral

Scholarship on the effects of increased flows of people, ideas, technologies, and goods associated with globalization (Appadurai 1996) has commonly concentrated on areas where the effects of this are abundant and easily visible, i.e., "the huge contemporary metropolis with its explosive and conspicuous diversity in people and languages, its hyper-mobility and constant flux" (Wang et al. 2014, 23). As Cornips and de Rooij (2018b) observe, however, it is unfounded how little attention has gone out to studying the effects of globalization in areas perceived as peripheral, where globalization has also altered daily life. This dissertation has contributed in addressing this gap by studying the discursive manifestation of globalization, in the form of ethnic categorization, in the relatively small and (from a national perspective) peripheralized city of Venlo.

Indeed, 28.4 percent of Venlo's inhabitants have a migration background compared to 23.6 percent in the Netherlands as a whole (Centraal Bureau voor de Statistiek 2019a). Venlo is furthermore Limburg's municipality with 
second most inhabitants with a so-called 'non-Western migration background.' In class $3 / 4 \mathrm{~b}$, about half of the pupils had a migration background and, as will have become evident by now, they regularly referred to this background in their daily interactional practices. Their diversity in terms of ethnic backgrounds was also recognizable in their language practices, as the linguistic resources of many pupils included languages other than Dutch. As such, the effects of globalization in terms of diversity could be observed among these pupils.

The expression this found in their practice of self-categorization certainly seemed to be affected by its embeddedness in this particular city and region. That is, whereas it has been found that in the larger Dutch cities, such as Amsterdam and Rotterdam, many people with migration background selfcategorize in terms of the city (thus referring to themselves as Amsterdammer or Rotterdammer) instead of as Nederlander - which seems to have become associated exclusively with people who are perceived as having no migration background (Slootman and Duyvendak 2015; Omlo 2011; van der Welle 2011; Özpamuk 2018) - I have not observed the use of a category such as Venlonaar or Limburger with that same 'mediating' effect (Slootman and Duyvendak 2015) among the pupils of class 3/4b. This may be explained by some specific characteristics of Venlo and Limburg. For instance, being located in the province of Limburg, which is often associated with a strong sense of local identity and particular sociocultural and linguistic practices, the membership category 'Limburger' or 'Venlonaar' may be less "open to diversity" (Slootman and Duyvendak 2015, 160) than, for instance, the category Amsterdammer - at least for this context and for these participants. This complements findings of previous sociolinguistic work in Limburg that has shown that although many people with a migration background in Limburg acquire local dialects, many are not comfortable using dialect because their authenticity and legitimacy as dialect-speakers is often contested (Cornips 2020a), and that people who are not perceived to look 'typically Limburgian' (e.g. because of their skin color) use dialect in conversation, they are often answered in standard Dutch (Thissen 2018).

Comparing the use and social positions of local dialects as well as immigrant languages' in one study can reveal how language policing becomes a site for discussing ideologies of belonging

The final contribution that merits being highlighted is that, in contrast to many studies in as well as outside the Netherlands that have focused either on the use of local dialects or on the use of 'immigrant languages' in the classroom, I have compared the use and indexicalities of both in this 
dissertation. This has enabled me to analyze the symbolic organization of languages and their speakers in class $3 / 4 \mathrm{~b}$, and to show how this symbolic organization reflects and reproduces wider patterns of social stratification and ever-changing societal concerns (cf. Nørreby and Madsen 2018).

A comparison of the position of dialects and 'immigrant languages' showed that, even though Limburgish dialects remain relegated to the informal sphere and generally lack a ratified position in schools, they no longer appear to be as stigmatized as they once were. Concerns about language as indicative of 'civilization,' which were at the root of the stigmatized position of dialects, have shifted to concerns about language and belonging. Today, in a sociopolitical climate wrought with discourse that problematizes people with migration backgrounds (Roggeband and van der Haar 2018), it is the languages associated with those people that receive the concern and rejection similar to that which once befell dialects. The patterns of linguistic policing that I observed in South High School - where, in general, teachers reinforced the standard Dutch language policy much more strictly when they heard pupils speak Turkish, Moroccan Arabic, or Berber in the classroom, than when pupils used dialect - reflect current Dutch society's concern with 'belonging,' and especially the question expressed in much political and popular discourse whether or not (descendants of) immigrants feel 'at home' in the Netherlands (Duyvendak, Reinders, and Wekker 2016). At the foundation of this were pervasive language ideologies, such as the idea that languages belong to people, who belong to places (Karrebæk 2013; Quist 2010), resulting in the idea that languages, and their speakers, can be seen to be 'in' or 'out of place' (Thissen 2018).

\subsection{Representativity and suggestions for further research}

This study has focused on the pupils of one particular school class, in one particular educational track, in one city in the Netherlands. This brings up the question of representativity and to what extent the findings in this dissertation are informative of other contexts. On the one hand, I would not claim that my case study of class 3/4b of South High School is representative of, for instance, 'vmbo pupils in Venlo' or 'people with migration backgrounds in the Netherlands.' On the contrary: I have emphasized throughout the dissertation that the meanings and functions of category labels cannot be taken for granted, but that they are locally defined and negotiated between individuals in a specific group composition, in a specific time and place. This study is thus unrepresentative from a strictly quantitative point of view, in that I have described meaning negotiations and functions of category labels and use of linguistic resources among the pupils and teachers of class 3/4b of South High 
School in Venlo in 2017-18, and these do not necessarily correspond to the meaning negotiations and functions of the same category labels, or language use, among current pupils in the same school, or among the same individuals by the time this dissertation is being read. This serves to underline that categorizations are negotiated between individuals and are specific to those individuals in their specific composition, at that time and in that place, and that their meanings can thus never be taken for granted but must be studied in context.

On the other hand, it would be unproductive to see this study merely as an unrepresentative case study of a number of pupils in one school class. It is not coincidental that I encountered these particular individuals in this classroom: pupils with migration backgrounds are overrepresented in vocational tracks in the Netherlands (Inspectie van het Onderwijs 2018), and this was also the case in class 3/4b at South High School. Furthermore, it is not coincidental that these pupils played with these particular categories. People with migration backgrounds, such as the pupils in this study and their families, are the subjects of much national political and societal debate about immigration and belonging. This discourse is reflected in the daily interactional practices of the pupils in the classroom that I observed. Additionally, the interactional practice of ethnic categorization does not appear to be limited to these pupils, as similar self-categorizations have also been reported among people of other age-groups and in other Dutch cities (e.g. Omlo 2011; Slootman 2018; van der Welle 2011). As the particular setting in which I studied ethnic categorization nevertheless undoubtedly influenced my findings, this study highlights interesting opportunities for further research, in particular by adapting certain elements of the research design to learn more about the dynamics and social meanings of ethnic categorization. I will offer five suggestions.

First, class $3 / 4 \mathrm{~b}$ included many pupils with migration backgrounds. It is possible that the quantitative dominance of these pupils in this peer-group, and in the vocational tracks of South High School in general, raised the prestige of assuming membership of the categories Marokkaan, Turk, or buitenlander (cf. Agirdag, van Houtte, and van Avermaet 2011). Future studies could assess whether the same or similar categorization practices occur in school classes with a smaller proportion of pupils with a family history of immigration.

Second, I conducted this study among pupils of the vocational tracks (vmbo basis and kader). In Chapter 3, I suggested that being enrolled in the undervalued vocational tracks and having a migration background meant that some pupils grappled with membership in two categories that are stigmatized in the Netherlands, and that their appropriation of the categories Marokkaan, 
Turk and buitenlander may be seen as a way for them to alleviate the stigmatizing load of at least one of the categories by which the outside world defined them. To investigate that proposition, a fruitful avenue for further research would be to conduct a similar study among pupils of the more prestigious pre-university educational tracks (vwo), to see whether those same category labels play a role among those pupils, or, if other kinds of categorizations are deployed, whether those also engage with the social positioning of these pupils.

Third, the pupils of class $3 / 4 \mathrm{~b}$ were between the ages $14-17$, which may limit the generalizability of the results to the larger population of Dutch people with a family history of migration. Adolescence is often seen as a period in which peer group affiliation becomes a central concern (e.g. Brown, Eicher, and Petrie 1986), and possibly, the strong tendency of the pupils in this study to categorize themselves in terms of ethnicity has to do with age-related variables (Sabatier 2008). There are indications that the phenomenon of selfcategorization as 'Moroccan' or 'Turk' itself is not limited to this age-group, however (Omlo 2011; Slootman 2018; Spotti 2014a; van der Welle 2011). What remains unclear, to my knowledge, is whether the interactional functions of references to these labels that I found among the pupils of class $3 / 4 \mathrm{~b}$ (such as jocular mockery or negotiation of power positions in interaction) correspond to those of people in other periods of their life. This could also be a fruitful avenue for further research.

Fourth, I suggested previously in this concluding chapter that the specific context of Venlo may have influenced the categorization practices of the pupils of class 3/4b. It would therefore be informative for further research to be conducted among a similar group of pupils in a dominant urban center that is associated more commonly with diversity in terms of descent, to see whether similar category labels play a role in these contexts, and if so, which meanings and interactional functions they have there.

Finally, this study has confined itself to an analysis of categorization and language practices in the context of formal schooling. Further research could take into account other important contexts in the lives of adolescents, such as sport clubs, neighborhood centers, or other places where youth hang out together. Other options would be to investigate categorization in their homes, or as part of their online life worlds, including social media.

\subsection{A final field note}

In keeping with promises, I would like to end this dissertation with a final transcript of interaction with a message from some participants of this study in which, incidentally, Hatice reminds us to pay attention to what ('secretly') 
goes on beyond first appearances, as I have aimed to do with my analyses of ethnic categories in this dissertation.

Pomme: $\quad$ Maar jongens! Ik heb nog één vraag

Amira: Ja? But guys! I have one more question

Pomme: $\quad$ Oké, ik ga dus mijn onderzoek opschrijven straks. Wat vinden jullie belangrijk dat daar wel of juist niet instaat?

Alright, so I am going to write up my research later. What do you find important for me to include, or to exclude?

Nouria: $\quad$ Wij zijn... geweldig

We are... amazing

Pomme: $\quad £$ Ja. Dat vind ik ook, echt waar. $£$

$£$ Yes. I agree, truly. $£$

Hatice: $\quad$ Misschien dat we fel reageren maar stiekem ${ }^{\circ}$ wel lief zijn ${ }^{\circ}$.

Maybe that we are blunt in our reactions, but that secretly, 'we are sweet. ${ }^{\circ}$

(Field notes, 13 March 2018, conversation with the group on my final day of fieldwork at South High School) 
202 Nederlanders and buitenlanders 


\section{References}

Agirdag, Orhan. 2010. "Exploring Bilingualism in a Monolingual School System: Insights from Turkish and Native Students from Belgian Schools." British Journal of Sociology of Education 31 (3): 307-21. https://doi.org/10.1080/01425691003700540.

Agirdag, Orhan, Mieke van Houtte, and Piet van Avermaet. 2011. "Ethnic School Context and the National and Sub-National Identifications of Pupils." Ethnic and Racial Studies 34 (2): 357-78. https://doi.org/10.1080/01419870.2010.510198.

American Anthropological Association. 2009. "Code of Ethics of the American Anthropological Association.”

American Anthropological Association. 2012. "Ethics Statement." 2012. http://ethics.americananthro.org/category/statement/.

Androutsopoulos, Jannis, and Alexandra Georgakopoulou. 2003. Discourse Constructions of Youth Identities. Amsterdam: John Benjamins.

Antaki, Charles, and Susan Widdicombe. 1998. "Identity as an Achievement and as a Tool." In Identities in Talk, edited by Charles Antaki and Susan Widdicombe, 1-14. London: Sage Publications.

Anthias, Floya. 2013. "Moving beyond the Janus Face of Integration and Diversity Discourses: Towards an Intersectional Framing." Sociological Review 61 (2): 323-43. https://doi.org/10.1111/1467-954X.12001.

Appadurai, Arjun. 1996. Modernity at Large: Cultural Dimensions of Globalization. Minneapolis: University of Minnesota Press.

Attardo, Salvatore, Jodi Eisterhold, Jennifer Hay, and Isabella Poggi. 2003. "Multimodal Markers of Irony and Sarcasm." Humor 16 (2): 243-60. https://doi.org/10.1515/humr.2003.012.

Auer, Peter. 2013. "The Geography of Language: Steps toward a New Approach." Freiberger Arbeitspapiere Zur Germanistischen Lingustik (FRAGL), no. 16: 139. http://portal.uni-freiburg.de/sdd/frag1/2013.16.

Augoustinos, Martha, and Danielle Every. 2010. "Accusations and Denials of Racism: Managing Moral Accountability in Public Discourse." Discourse and Society 21 (3): 251-56. https://doi.org/10.1177/0957926509360650.

Aukrust, Vibeke Grøver, and Veslemøy Rydland. 2009. “Does It Matter?’ Talking about Ethnic Diversity in Preschool and First Grade Classrooms." Journal of Pragmatics 41 (8): 1538-56. https://doi.org/10.1016/j.pragma.2007.03.009.

Azghari, Youssef, Erna Hooghiemstra, and Fons J. R. van de Vijver. 2015. "Young Moroccan-Dutch: Thinking in Dutch, Feeling Moroccan." Journal of Muslim Minority Affairs 35 (2): 280-95. https://doi.org/10.1080/13602004.2015.1052666.

Baker, Carolyn. 1997. "Ticketing Rules: Categorization and Moral Ordering in a School Staff Meeting." In Culture in Action: Studies in Membership 
Categorization Analysis, edited by Stephen Hester and Peter Eglin, 77-98. Washington, DC: International Institute for Ethnomethodology and Conversation Analysis \& University Press of America.

Bakhtin, Mikael. 1981. The Dialogic Imagination. Austin: University of Texas Press. Barth, Fredrik. 1969. "Introduction." In Ethnic Groups and Boundaries. The Social Organization of Culture Difference, 9-38. https://doi.org/10.2307/588416.

Baumann, Gerd, and Andre Gingrich. 2004. Grammars of Identity/Alterity. A Structural Approach. New York: Berghahn Books.

Benwell, Bethan, and Elizabeth Stokoe. 2006. Discourse and Identity. Edinburgh: Edinburgh University Press. https://doi.org/10.1080/13670050802149457.

Bergh, L. van den, E. Denessen, L. Hornstra, M. Voeten, and R. W. Holland. 2010. "The Implicit Prejudiced Attitudes of Teachers: Relations to Teacher Expectations and the Ethnic Achievement Gap." American Educational Research Journal 47 (2): 497-527. https://doi.org/10.3102/0002831209353594.

Berreman, Gerald D. 2012. "Ethics versus 'Realism' in Anthropology.” In Ethnographic Fieldwork: An Anthropological Reader, edited by Antonius C.G.M. Robben and Jeffrey A. Sluka, Second edi, 331-52. Malden, MA: Wiley-Blackwell.

Bezcioglu-Göktolga, Irem, and Kutlay Yagmur. 2018. "The Impact of Dutch Teachers on Family Language Policy of Turkish Immigrant Parents.” Language, Culture and Curriculum 31 (3): 220-34. https://doi.org/10.1080/07908318.2018.1504392.

Bezemer, Jeff. 2003. Dealing with Multilingualism in Education: A Case Study of a Dutch Primary School Classroom. Amsterdam: Aksant.

Bezemer, Jeff. 2007. “They Don't Have That Feeling'. The Attribution of Linguistic Resources to Multilingual Students in a Primary School." Linguistics and Education 18 (1): 65-78. https://doi.org/10.1016/j.linged.2007.03.001.

Bezemer, Jeff, and Sjaak Kroon. 2006. "'You Don't Need to Know the Turkish Word': Immigrant Minority Language Teaching Policies and Practice in the Netherlands." L1 - Educational Studies in Language and Literature 6 (1): 13-29.

Billig, Michael. 1996. Arguing and Thinking: A Rhetorical Approach to Social Psychology. Second edi. Cambridge: Cambridge University Press.

Billig, Michael. 1999. "Whose Terms? Whose Ordinariness? Rhetoric and Ideology in Conversation Analysis." Discourse E Society 10 (4): 543-82.

Billig, Michael. 2005. Laughter and Ridicule. Towards a Social Critique of Humour. London: Sage.

Blommaert, Jan, and Ben Rampton. 2011. "Language and Superdiversity." Diversities 13 (2): 1-22.

Blumer, Herbert. 1969. Symbolic Interactionism. New York: Prentice Hall.

Borneman, John, and Abdellah Hammoudi. 2009. "The Fieldwork Encounter, Experience, and the Making of Truth: An Introduction." In Being There: The Fieldwork Encounter and the Making of Truth, edited by John Borneman and Abdellah Hammoudi, 1-24. Berkeley and Los Angeles: University of California Press. 
Bouabid, Abdessamad. 2016. "De Marokkanenpaniek: De Sociale Constructie van 'Marokkanen' Als Folk Devils.” Tijdschrift over Cultuur \& Criminaliteit 6 (1): 82-99. https://doi.org/10.5553/TCC/221195072016006001006.

Bouras, Nadia. 2012. Het Land van Herkomst: Perspectieven Op Verbondenheid Met Marokko, 1960-2010. PhD diss., University of Leiden. https://openaccess.leidenuniv.n1/handle/1887/20180

—. 2013. "Shifting Perspectives on Transnationalism: Analysing Dutch Political Discourse on Moroccan Migrants' Transnational Ties, 1960-2010.” Ethnic and Racial Studies 36 (7): 1219-31. https://doi.org/10.1080/01419870.2013.785580.

Bovens, Mark, Meike Bokhorst, Roel Jennissen, and Godfried Engbersen. 2016. "Migratie En Classificatie: Naar Een Meervoudig Migratie-Idioom." Den Haag: Wetenschappelijke Raad voor het Regeringsbeleid. http://www.wrr.nl/fileadmin/nl/publicaties/PDFverkenningen/V034_Migratie_en_classificatieDEF.pdf.

Bozay, Kemal. 2012. "Probleme Und Ursachen Der Re-Ethnisierung Und Selbstethnisierung Im Klassenzimmer." In Das Interkulturelle Lebrerzimmer. Perspektiven Neuer Deutscher Lehrkräfte Auf Den Bildungs- Und Integrationsdiskurs., edited by Karim Fereidooni, 117-24. Dorsten, Deutschland: Springer.

Briggs, Charles L. 1986. Learning How to Ask: A Sociolinguistic Appraisal of the Role of the Interview in Social Science Research. Cambridge: Cambridge University Press.

Brown, B. Bradford, Sue Ann Eicher, and Sandra Petrie. 1986. "The Importance of Peer Group ('crowd') Affiliation in Adolescence." Journal of Adolescence 9 (1): 73-96. https://doi.org/10.1016/S0140-1971(86)80029-X.

Brubaker, Rogers. 2002. "Ethnicity without Groups." European Journal of Sociology 43 (2): 163-89. https://doi.org/10.1017/S0003975602001066.

Brubaker, Rogers. 2004. Ethnicity without Groups. Cambridge, Massachusetts: Harvard University Press.

Brubaker, Rogers. 2009. "Ethnicity, Race, and Nationalism." Annual Review of Sociology 35 (1): 21-42. https://doi.org/10.1146/annurev-soc-070308-115916.

Brubaker, Rogers, and Frederick Cooper. 2000. "Beyond 'Identity." Theory and Society 29 (1): 1-47. https://doi.org/10.1023/A:1007068714468.

Brubaker, Rogers, Mara Loveman, and Peter Stamatov. 2004. "Ethnicity as Cognition." Theory and Society 33 (1): 31-64. https://doi.org/10.1023/B:RYSO.0000021405.18890.63.

Bucholtz, Mary. 2011. White Kids. Language, Race, and Styles of Youth Identity. New York: Cambridge University Press.

Bucholtz, Mary, and Kira Hall. 2004. "Language and Identity." In A Companion to Linguistic Antbropology, edited by Alessandro Duranti, 369-94. Malden, MA: Blackwell Publishing. https://doi.org/10.1002/9780470996522.ch16.

Bucholtz, Mary, and Kira Hall. 2005. "Identity and Interaction: A Sociocultural Linguistic Approach." Discourse Studies 7 (4-5): 585-614. https://doi.org/10.1177/1461445605054407. 


\section{Nederlanders and buitenlanders}

Bucholtz, Mary, and Kira Hall. 2008. "All of the above: New Coalitions in Sociocultural Linguistics." Journal of Sociolinguistics 12 (4): 401-31.

Bucholtz, Mary, and Elena Skapoulli. 2009. "Youth Language at the Intersection: From Migration to Globalization." Pragmatics 19 (1): 1-16. https://doi.org/10.1075/prag.19.1.01buc.

Buitelaar, Marjo, and Femke Stock. 2010. "Making Homes in Turbulent Times: Moroccan-Dutch Muslims Contesting Dominant Discourses of Belonging." In Muslim Diaspora in the West. Negotiating Gender, Home and Belonging, edited by Haideh Moghissi and Halleh Ghorashi, 165-79. Abingdon, Oxon: Routledge.

Bulk, Lenie van den. 2011. "Later Kan Ik Altijd Nog Worden Wat Ik Wil: Statusbeleving, Eigenwaarde En Toekomstbeeld van Leerlingen in Het Voortgezet Onderwijs, Met de Nadruk Op de Relatieve Positie van VmboLeerlingen."

Butter, Ewoud. 2019. "Nederlanders, Buitenlanders, 'Allochtonen'. De Cijfers.” Republiekallochtonie.Nl, 2019. http://www.republiekallochtonie.nl/blog/feiten/nederlanders-buitenlandersallochtonen-de-cijfers.

Camps, Diana M.J. 2017. "Legitimating Limburgish: The Reproduction of Heritage.” In Standardizing Minority Languages: Competing Ideologies of Authority and Authenticity in the Global Periphery, 66-83. Routledge.

Camps, Diana M.J. 2018. "Legitimating Limburgish: The Discursive Construction of a Regional Language in the Netherlands." PhD diss., University of Oslo.

CBS Statline. 2018. "Jongeren (0 Tot 25 Jaar); Geslacht, Leeftijd, Migratieachtergrond, Regio's." Centraal Bureau voor de Statistiek. https://jeugdstatline.cbs.n1/Jeugdmonitor/publication/?DM=SLNL\&PA=710 $09 \mathrm{NED} \& \mathrm{D} 1=0 \& \mathrm{D} 2=0 \& \mathrm{D} 3=0 \& \mathrm{D} 4=\mathrm{a} \& \mathrm{D} 5=0 \& \mathrm{D} 6=0,5,10-$ $1 \& H D R=T, G 4, G 1, G 2, G 5 \& S T B=G 3 \& C H A R T T Y P E=1 \& V W=T$.

Centraal Bureau voor de Statistiek. 2019a. “CBS Statline.” 2019. https://opendata.cbs.nl/statline/\#/CBS/nl/dataset/70072ned/table?ts=1574414 450549 .

Centraal Bureau voor de Statistiek. 2019b. "Hoeveel Mensen Met Een Migratieachtergrond Wonen in Nederland?" 2019. https://www.cbs.nl/nln1/dossier/dossier-asiel-migratie-en-integratie/hoeveel-mensen-met-eenmigratieachtergrond-wonen-in-nederland-.

Chun, Elaine. 2004. “Ideologies of Legitimate Mockery: Margaret Cho's Revoicings of Mock Asian." Pragmatics 14 (2-3): 263-89. https://doi.org/10.1075/prag.14.2-3.10chu.

Chun, Elaine. 2011. "Reading Race beyond Black and White." Discourse E Society 22 (4): 403-21. https://doi.org/10.1177/0957926510395833.

Clemente, Ignasi. 2013. "Conversation Analysis and Anthropology." In The Handbook of Conversation Analysis, edited by Jack Sidnell and Tanya Stivers, 688-700. Malden, MA: Wiley-Blackwell.

Clift, Rebecca. 1999. "Irony in Conversation." Language in Society 28 (4): 523-53. https://doi.org/10.1017/s0047404599004029. 
Collins, James. 2017. "Dilemmas of Race, Register, and Inequality in South Africa." Language in Society 46 (1): 39-56. https://doi.org/10.1017/s004740451600083x.

Copland, Fiona, and Angela Creese. 2015. "Linguistic Ethnography.” In Linguistic Ethnography: Collecting, Analysing and Presenting Data, edited by Fiona Copland and Angela Creese, 12-27. London: Sage.

Cornips, Leonie. 2002. "Etnisch Nederlands in Lombok." In Een Buurt in Beweging. Talen En Culturen in Het Utrechtse Lombok En Transvaal, edited by Hans Bennis, Guus Extra, Pieter Muysken, and Jacomine Nortier, 285-301. Amsterdam: Stichting Beheer IISG.

Cornips, Leonie. 2008. "Loosing Grammatical Gender in Dutch: The Result of Bilingual Acquisition and/or an Act of Identity?" International Journal of Bilingualism 12 (1-2): 105-24. https://doi.org/10.1177/13670069080120010701.

Cornips, Leonie. 2012. Eigen En Vreemd: Meertaligheid in Nederland. Amsterdam: Amsterdam University Press.

Cornips, Leonie. 2020a. "Dialect Acquisition by 'New Speakers' of Dutch and Their Linguistic Othering." Journal of Multilingual and Multicultural Development: 1-17. https://doi.org/10.1080/01434632.2020.1730384.

Cornips, Leonie. 2020b. “The Impact of Preschool Attendance on Child's Bidialectism in the Netherlands. Why Toddlers May Stop Speaking a Regional Language (Limburgish) at Home.” Language in Society 49 (3): 333355. https://doi.org/10.1017/S0047404520000275.

Cornips, Leonie, Jürgen Jaspers, and Vincent de Rooij. 2014. "The Politics of Labeling Youth Vernaculars in the Netherlands and Belgium." In Language, Youth and Identity in the 21st Century. Linguistic Practices across Urban Spaces, 45-70. Cambridge University Press. https://doi.org/10.1017/CBO9781139061896.004.

Cornips, Leonie, and Ad Knotter. 2016. "De Uitvinding van Limburg: De Territorialisering van Geschiedenis, Taal en Identiteit." In Studies over de Sociaal-Economische Geschiedenis van Limburg. Jaarboek Sociaal Historisch Centrum Voor Limburg, Volume LXI: 136-163., edited by Ad Knotter and W. Rutten, 136-63. Maastricht: Sociaal Historisch Centrum voor Limburg.

Cornips, Leonie, and Vincent de Rooij. 2013. "Selfing and Othering through Categories of Race, Place, and Language among Minority Youths in Rotterdam, The Netherlands." In Multilingualism and Language Diversity in Urban Areas. Acquisition, Identities, Space, Education, edited by Peter Siemund, Ingrid Gogolin, Monika Edith Schulz, and Julia Davydova, 129-64. John Benjamins Publishing Company.

Cornips, Leonie, and Vincent de Rooij. 2015. "Belonging through Languagecultural Practices in the Periphery: The Politics of Carnival in the Dutch Province of Limburg." Anthropological Journal of European Cultures 24 (1): 83-101. https://doi.org/10.3167/ajec.2015.240106.

Cornips, Leonie, and Vincent de Rooij. 2018a. "Introduction: Belonging through Linguistic Place-Making in Center-Periphery Constellations.” In The 
Sociolinguistics of Place and Belonging. Perspectives from the Margins, edited by Leonie Cornips and Vincent de Rooij, 1-16. John Benjamins Publishing Company.

Cornips, Leonie, and Vincent de Rooij. 2018b. The Sociolinguistics of Place and Belonging. Perspectives from the Margins. Amsterdam: John Benjamins Publishing Company.

Cornips, Leonie, Vincent de Rooij, and Irene Stengs. 2012. "Carnavalesk Taalgebruik En de Constructie van Lokale Identiteiten: Een Pleidooi Voor Taalcultuur Als Onderzoeksveld." Dutch Journal of Applied Linguistics 1 (1): 15-40. https://doi.org/10.1075/dujal.1.1.04cor.

Cornips, Leonie, Vincent de Rooij, Irene Stengs, and Lotte Thissen. 2016. "Dialect and Local Media. Reproducing the Multi-Dialectal Space in Limburg (the Netherlands)." In Style, Media and Language Ideologies, SLICE Series Norwegian Novus Style, edited by Jacob Thøgersen, Nicolas Coupland, and Janus Mortensen, 189-216. Novus AS.

Coupland, Nikolas. 2007. Style: Language Variation and Identity. Cambridge: Cambridge University Press.

Coupland, Nikolas. 2011. "The Sociolinguistics of Style." In The Cambridge Handbook of Sociolinguistics, edited by Rajend Mesthrie, 138-56. Cambridge: Cambridge University Press.

Cranssen, Tanja. 2003. "Marokkaanse Mijnwerkers in Limburg." In Jaarboek van Het Sociaal Historisch Centrum Voor Limburg, Deel XLVIII, edited by Ad Knotter and Willibrord Rutten, 121-48. Maastricht: Sociaal Historisch Centrum voor Limburg.

Creese, Angela. 2008. "Linguistic Ethnography." In Encyclopedia of Language and Education, edited by K.A. King and N.H. Hornberger, 2nd ed., 10:229-41. Springer.

Creese, Angela, and Fiona Copland. 2017. "Linguistic Ethnography." In Research Methods in Language and Education, edited by Kendall A. King, Yi-Ju Lai, and Stephen May, Third edit, 339-51. Cham, Switzerland: Springer. https://doi.org/10.1007/978-3-319-02249-9.

D’Amato, John. 1987. “The Belly of the Beast: On Cultural Differences, Castelike Status, and the Politics of School." Anthropology \& Education Quarterly 18 (4): $357-60$.

Daalen, Rineke van. 2010. Het Vmbo Als Stigma. Lessen, Leerlingen En Gestrande Idealen. Amsterdam: Uitgeverij Augustus.

Dagevos, Jaco, and Willem Huijnk. 2014. "Segmentatie Langs Etnische Grenzen." In Verschil in Nederland: Sociaal En Cultureel Rapport 2014, edited by C. Vrooman, M. Gijsberts, and J. Boelhouwer, 253-80. Den Haag: Sociaal en Cultureel Planbureau.

Deppermann, Arnulf, and Axel Schmidt. 2000. "Disrespecting: A Conversational Practice for the Negotiation of Status in Juvenile Peer-Groups." Pragmatics in 2000: Selected Papers from the 7th International Pragmatics Conference, 156-64.

Derbali, Naïm. 2018. "Hoe Het Woord 'Mocro' Zijn Onschuld Verloor.” Trouw, April 13, 2018. https://www.trouw.nl/samenleving/hoe-het-woord-mocro- 
zijn-onschuld-verloor a827fe4e/.

Dietze, Gabriele, Elahe Haschemi Yekani, and Beatrice Michaelis. 2019. "Modes of Being vs. Categories." In Beyond Gender: An Advanced Introduction to Futures of Feminist and Sexuality Studies, edited by Greta Olson, Daniel Hartley, Mirjam Horn-Schott, and Leonie Schmidt, 117-33. London and New York: Routledge.

Dijk, Teun Van. 1993. “Denying Racism: Elite Discourse and Racism.” In Racism and Migration in Western Europe, edited by John Solomos and John Wrench, 179-93. Oxford: Berg. https://doi.org/10.1016/0147-1767(94)90010-8.

Dirks, Bart. 2018. "Bewoners Woonwagenkampen Willen Plekken Voor Hun Kinderen: 'Ze Willen Ons in Stenen Gevangenissen Douwen, Maar Ik Zit Nog Liever in Een Echte Cel.” De Volkskrant, 2018.

https://www.volkskrant.nl/nieuws-achtergrond/bewonerswoonwagenkampen-willen-plekken-voor-hun-kinderen-ze-willen-ons-instenen-gevangenissen-douwen-maar-ik-zit-nog-liever-in-een-echtecel b9f3168b/.

Dorleijn, Margreet, Maarten Mous, and Jacomine Nortier. 2015. "Urban Youth Speech Styles in Kenya and the Netherlands.” In Language, Youth and Identity in the 21st Century. Linguistic Practices across Urban Spaces, edited by Jacomine Nortier and Bente A. Svendsen, 271-89. Cambridge: Cambridge University Press.

Drew, Paul. 1987. "Po-Faced Receipts of Teases.” Linguistics 25 (1): 219-53. https://doi.org/10.1515/ling.1987.25.1.219.

Driessen, Geert. 2009. "Houdoe? Afscheid van Streektalen En Dialecten?” In Dialect, van Schoot Tot School?, edited by Roeland Van Hout, Veronique De Tier, and Jos Swanenberg. Groesbeek: Stichting Nederlandse dialecten.

Duchêne, Alexandre, and Monica Heller. 2012. Language in Late Capitalism: Pride and Profit. Routledge. https://doi.org/10.4324/9780203155868.

Duff, Patricia A. 2002. "The Discursive Co-Construction of Knowledge, Identity, and Difference: An Ethnography of Communication in the High School Mainstream." Applied Linguistics 23 (3): 289-322. https://doi.org/10.1093/applin/23.3.289.

Duranti, Alessandro. 1997. Linguistic Antbropology. Cambridge: Cambridge University Press.

Duranti, Alessandro. 2003. "Language as Culture in U.S. Anthropology: Three Paradigms." Current Antbropology 3 (3): 323-47. https://doi.org/10.1075/babel.10.1.08god.

Duranti, Alessandro. 2011. "Linguistic Anthropology: The Study of Language as a Non-Neutral Medium." In The Cambridge Handbook of Sociolinguistics, edited by Rajend Mesthrie, 28-46. Cambridge: Cambridge University Press.

Duyvendak, Jan Willem. 2011. The Politics of Home. Belonging and Nostalgia in Western Europe and the United States. Palgrave Macmillan. https://doi.org/10.1057/9780230305076.

Duyvendak, Jan Willem, Leeke Reinders, and Fenneke Wekker. 2016. "Homing the Dutch. An Introduction.” Home Cultures 13 (2): 87-100. 
https://doi.org/10.1080/17406315.2016.1190584.

Dynel, Marta. 2009. "Beyond a Joke: Types of Conversational Humour." Linguistics and Language Compass 3 (5): 1284-99. https://doi.org/10.1111/j.1749818X.2009.00152.x.

Eckert, Penelope. 1989. Jocks and Burnouts: Social Categories and Identity in the High School. New York: Teachers College Press.

Eckert, Penelope. 2012. "Three Waves of Variation Study: The Emergence of Meaning in the Study of Sociolinguistic Variation." Annual Review of Anthropology 41 (June): 87-100. https://doi.org/10.1146/annurev-anthro092611-145828.

Edwards, Derek. 1991. "Categories Are for Talking: On the Cognitive and Discursive Bases of Categorization." Theory \& Psychology 1 (4): 515-42.

Edwards, Derek. 1998. "The Relevant Thing about Her: Social Identity Categories in Use." In Identities in Talk, edited by Sue Widdicombe and Charles Antaki, 15-33. London: Sage Publications.

Eijberts, Melanie, and Halleh Ghorashi. 2017. "Biographies and the Doubleness of Inclusion and Exclusion." Social Identities 23 (2): 163-78. https://doi.org/10.1080/13504630.2016.1244766.

Enfield, N. J., Tanya Stivers, Penelope Brown, Christina Englert, Katariina Harjunpää, Makoto Hayashi, Trine Heinemann, et al. 2019. "Polar Answers." Journal of Linguistics 55 (2): 277-304. https://doi.org/10.1017/s0022226718000336.

Englert, Christina. 2010. "Questions and Responses in Dutch Conversations." Journal of Pragmatics 42 (10): 2666-84. https://doi.org/10.1016/j.pragma.2010.04.005.

Ersanilli, Evelyn, and Ruud Koopmans. 2010. "Rewarding Integration? Citizenship Regulations and the Socio-Cultural Integration of Immigrants in the Netherlands, France and Germany." Journal of Ethnic and Migration Studies 36 (5): 773-91. https://doi.org/10.1080/13691831003764318.

Evaldsson, Ann-Carita. 2005. "Staging Insults and Mobilizing Categorizations in a Multiethnic Peer Group.” Discourse \& Society 16 (6): 763-86. https://doi.org/10.1177/0957926505056663.

Evaldsson, Ann-Carita, and Asta Cekaite. 2010. “'Schwedis He Can't Even Say Swedish'-Subverting and Reproducing Institutionalized Norms for Language Use in Multilingual Peer Groups." Pragmatics 20 (4): 587-604. https://doi.org/10.1075/prag.20.4.05eva.

Extra, Guus, and Kutlay Yağmur. 2012. Language Rich Europe: Trends in Policies and Practices for Multilingualism in Europe. Cambridge University Press.

Extra, Guus, and Kutlay Yaĝmur. 2006. "Immigrant Minority Languages at Home and at School: A Case Study of the Netherlands." European Education 38 (2): 50-63. https://doi.org/10.2753/eue1056-4934380204.

Extra, Guus, and Kutlay Yaĝmur. 2010. "Language Proficiency and Socio-Cultural Orientation of Turkish and Moroccan Youngsters in the Netherlands."

Language and Education 24 (2): 117-32. https://doi.org/10.1080/09500780903096561. 
Fine, Gary Alan, and Michaela D E Soucey. 2005. "Joking Cultures: Humor Themes as Social Regulation in Group Life." Humor 18 (1): 1-22.

Ford, Cecilia E., and Barbara A. Fox. 2011. "Multiple Practices for Constructing Laughables." In Prosody in Interaction, edited by Dagmar Barth-Weingarten, Elisabeth Reber, and Margret Selting, 339-68. Amsterdam: John Benjamins Publishing Company. https://doi.org/10.1075/sidag.23.27for.

Francis, David, and Stephen Hester. 2004. An Invitation to Ethnomethodology: Language, Society and Social Interaction. London: Sage Publications.

Geschiere, Peter. 2009. "Autochthony in Europe: The Dutch Turn." In The Perils of Belonging, 130-68. Chicago \& London: University of Chicago Press. https://doi.org/10.7208/chicago/9780226289663.003.0005.

Ghorashi, Halleh. 2006. "When Is My Dutch Good Enough? Experiences of Refugee Women with Dutch Labour Organizations." Journal of International Migration and Integration 7 (1): 51-70. https://doi.org/10.1007/s12134-0061002-4.

Ghorashi, Halleh. 2017. "Negotiating Belonging beyond Rootedness: Unsettling the Sedentary Bias in the Dutch Culturalist Discourse." Ethnic and Racial Studies 40 (14): 2426-43. https://doi.org/10.1080/01419870.2016.1248462.

Goffman, Erving. 1981. Forms of Talk. Philadelphia: University of Pennsylvania Press.

Goodwin, Marjorie Harness. 1990. He-Said-She-Said: Talk as Social Organization Among Black Children. Bloomington and Indianapolis: Indiana University Press.

Goodwin, Marjorie Harness. 2002. “Building Power Asymmetries in Girls' Interaction.” Discourse E Society 13 (6): 715-30. https://doi.org/10.1177/0957926502013006752.

Goodwin, Marjorie Harness. 2006. The Hidden Life of Girls: Games of Stance, Status, and Exclusion. Malden, MA: Blackwell Publishing.

Grondelaers, Stefan, Roeland van Hout, and Mieke Steegs. 2010. "Evaluating Regional Accent Variation in Standard Dutch.” Journal of Language and Social Psychology 29 (1): 101-16. https://doi.org/10.1177/0261927X09351681.

Gumperz, John J. 1982. Discourse Strategies. Cambridge: Cambridge University Press.

Günthner, Susanne. 2011. "The Dynamics of Communicative Practices in Transmigrational Contexts: 'Insulting Remarks' and 'Stylized Category Animations' in Everyday Interactions among Male Youth in Germany." Text and Talk 31 (4): 447-73. https://doi.org/10.1515/TEXT.2011.022.

Haar, Marleen van der, and Dvora Yanow. 2011. "Allochtoon Als Metafoor En Categorie: Over de Handelingsimplicaties van Beleidstaal.” Beleid En Maatschappij 38 (2): 160-78.

Halonen, Mia, and Sari Pietikäinen. 2017. "Mocking Fakeness: Performance, Phonetic Aspiration and Ethnic Humour." Pragmatics 27 (4): 507-28. https://doi.org/10.1075/prag.27.4.02hal.

Hamidi, Lotfi El. 2018. "Nederlander, Een Besmeurd Begrip.” NRC, 2018. https://www.nrc.nl/nieuws/2018/10/28/nederlander-een-besmeurd-begrip- 


\section{Nederlanders and buitenlanders}

a2753079.

Hansen, Alan D. 2005. "A Practical Task: Ethnicity as a Resource in Social Interaction." Research on Language E Social Interaction 38 (1): 63-104. https://doi.org/10.1207/s15327973rlsi3801_3.

Hanushek, Eric A., and Ludger Wößmann. 2006. "Does Early Tracking Affect Educational Inequality and Performance? Differences-in-Differences Evidence across Countries." Economic Journal 116 (115): C63-76. https://doi.org/10.1111/j.1468-0297.2006.01076.x.

Haugh, Michael. 2010. "Jocular Mockery, (Dis)Affiliation, and Face." Journal of Pragmatics 42 (8): 2106-19. https://doi.org/10.1016/j.pragma.2009.12.018.

Haugh, Michael. 2016. "Just Kidding': Teasing and Claims to Non-Serious Intent." Journal of Pragmatics 95: 120-36. https://doi.org/10.1016/j.pragma.2015.12.004.

Herweijer, Lex. 2008. "Gestruikeld Voor de Start: De School Verlaten Zonder Startkwalificatie." Den Haag.

Hester, Stephen, and Peter Eglin. 1997. "Membership Categorization Analysis: An Introduction." In Culture in Action. Studies in Membership Categorization Analysis, edited by Stephen Hester and Peter Eglin, 1-24. Washington, DC: International Institute for Ethnomethodology and Conversation Analysis \& University Press of America.

Hester, Stephen, and David Francis. 2004. An Invitation to Ethnomethodology: Language, Society and Interaction.

Holmes, Janet, and Meredith Marra. 2002. "Over the Edge? Subversive Humor between Colleagues and Friends.” Humor 15 (1): 65-87. https://doi.org/10.1515/humr.2002.006.

Hout, Roeland van. 1999. 'Waarom Veroveren 'hun' Onze Taal? Sociale En Taalkundige Verklaringen Voor de Opkomst van Een Subjectspronomen.” Toegepaste Taalwetenschap in Artikelen 62 (1): 73-86.

Houtte, Mieke Van, and Peter A.J. Stevens. 2010. "The Culture of Futility and Its Impact on Study Culture in Technical/Vocational Schools in Belgium." Oxford Review of Education 36 (1): 23-43. https://doi.org/10.1080/03054980903481564.

Huijnk, Willem, and Iris Andriessen. 2016. "Integratie in Zicht? De Integratie van Migranten in Nederland Op Acht Terreinen Nader Bekeken." Den Haag.

Huijnk, Willem, and Jaco Dagevos. 2012. "Dichter Bij Elkaar? De SociaalCulturele Positie van Niet-Westerse Migranten in Nederland.” Den Haag. https://doi.org/10.1007/s12479-014-00113-4.

Huuki, Tuija, Sari Manninen, and Vappu Sunnari. 2010. "Humour as a Resource and Strategy for Boys to Gain Status in the Field of Informal School." Gender and Education 22 (4): 369-83. https://doi.org/10.1080/09540250903352317.

Inspectie van het Onderwijs. 2018. "Onderwijsverslag: De Staat van Het Onderwijs 2016/2017.” Ministerie van Onderwijs, Cultuur En Wetenschap. https://www.onderwijsinspectie.n1/documenten/rapporten/2018/04/11/rappor t-de-staat-van-het-onderwijs.

Jaspers, Jürgen. 2005a. "Linguistic Sabotage in a Context of Monolingualism and 
Standardization." Language and Communication 25: 279-97.

https://doi.org/10.1016/j.langcom.2005.03.007.

Jaspers, Jürgen. 2005b. Tegenwerken, belachelijk doen. Talige sabotage van Marokkaanse jongens op een Antwerpse middelbare school. Een sociolinguïstische etnografie. Brussels: VUB press.

Jaspers, Jürgen. 2008. "Problematizing Ethnolects: Naming Linguistic Practices in an Antwerp Secondary School." International Journal of Bilingualism 12 (1/2): 85-103. https://doi.org/10.1177/13670069080120010601.

Jaspers, Jürgen. 2011a. "Strange Bedfellows: Appropriations of a Tainted Urban Dialect." Journal of Sociolinguistics 15 (4): 493-524. https://doi.org/10.1111/j.1467-9841.2011.00502.x.

Jaspers, Jürgen. 2011b. "Talking like a 'Zerolingual': Ambiguous Linguistic Caricatures at an Urban Secondary School." Journal of Pragmatics 43 (5): 1264-78. https://doi.org/10.1016/j.pragma.2010.05.012.

Jaspers, Jürgen. 2014. "Stylisations as Teacher Practice." Language in Society 43: 371-93. https://doi.org/10.1017/S0047404514000360.

Jaspers, Jürgen. 2015. "Tussen Meervoudige Vuren: De Implementatie van Eentalig Taalbeleid in Een Context van Taaldiversiteit." Pedagogische Studiën 92: 34460.

Jaspers, Jürgen. 2017. "Language Education Policy and Sociolinguistics: Toward a New Critical Engagement." In The Oxford Handbook on Language Policy and Planning, edited by M. Pérez-Milans and J. Tollefson. Oxford: Oxford University Press.

Jaspers, Jürgen. 2019. "The Deliberative Teacher: Wavering between Linguistic Uniformity and Diversity." In Critical Perspectives on Linguistic Fixity and Fluidity: Languagised Lives, 217-40. New York: Routledge.

Jaspers, Jürgen, and Sarah Van Hoof. 2019. “Style and Stylisation.” In The Routledge Handbook of Linguistic Ethnography, edited by Karin Tusting. London and New York: Routledge.

Jaspers, Jürgen, and Kirsten Rosiers. 2019. "Soft Power: Teachers' Friendly Implementation of a Severe Monolingual Policy." Journal of Multilingual and Multicultural Development 0 (0): 1-14. https://doi.org/10.1080/01434632.2019.1585864.

Jefferson, Gail. 1979. "A Technique for Inviting Laughter and Its Subsequent Acceptance/Declination.” In Everyday Language: Studies in Ethnomethodology, edited by George Psathas, 79-95. New York: Irvington.

Jefferson, Gail. 1984. "Notes on Some Orderlinesses of Overlap Onset." In Discourse Analysis and Natural Rhetorics, 11-38. Padova, Italy: CLEUP Editore. http://www.liso.ucsb.edu/Jefferson/overlap_onset.pdf.

Jefferson, Gail. 2004. "A Note on Laughter in 'Male-Female' Interaction." Discourse Studies 6 (1): 117-33. https://doi.org/10.1177/1461445604039445.

Jefferson, Gail, Harvey Sacks, and Emanuel A. Schegloff. 1987. "Notes on Laughter in the Pursuit of Intimacy." In Talk and Social Organization, edited by G. Button and J.R.E. Lee, 152-205. Clevedon, UK: Multilingual Matters.

Jenkins, Richard. 2008. Rethinking Ethnicity: Arguments and Explorations. Second 
ed. London: Sage Publications. https://doi.org/10.4135/9781446214855.

Jennissen, Roel, Godfried Engbersen, Meike Bokhorst, and Mark Bovens. 2018.

"De Nieuwe Verscheidenheid. Toenemende Diversiteit Naar Herkomst in

Nederland.” Den Haag: Wetenschappelijke Raad voor het Regeringsbeleid.

Jonsson, Rickard. 2018. "Swedes Can't Swear: Making Fun at a Multiethnic

Secondary School." Journal of Language, Identity \& Education 17 (5): 320-35. https://doi.org/10.1080/15348458.2018.1469412.

Jørgensen, Jens Normann. 2005. "Plurilingual Conversations among Bilingual

Adolescents." Journal of Pragmatics 37 (3): 391-402.

https://doi.org/10.1016/j.pragma.2004.10.009.

Jørgensen, Jens Normann, Martha Karrebæk, Lian Malai Madsen, and Janus

Spindler Møller. 2011. "Polylanguaging in Superdiversity.” Diversities 13 (2): 23-37. https://doi.org/10.4324/9781315730240.

Jurva, Katrina, and Inga Jasinskaja-Lahti. 2015. “Accounting for a Planned Migration through Ethnic Identity Talk.” Culture and Psychology 21 (2): 27689. https://doi.org/10.1177/1354067X15570486.

Karrebæk, Martha Sif. 2013. "Don't Speak like That to Her!': Linguistic Minority Children's Socialization into an Ideology of Monolingualism." Journal of Sociolinguistics 17 (3): 355-75. https://doi.org/10.1111/josl.12035.

Keltner, Dacher, Lisa Capps, Ann M. Kring, Randall C. Young, and Erin A. Heerey. 2001. "Just Teasing: A Conceptual Analysis and Empirical Review." Psychological Bulletin 127 (2): 229-48. https://doi.org/10.1037/00332909.127.2.229.

Ketner, Susan L., Marjo W. Buitelaar, and Harke A. Bosma. 2004. "Identity Strategies Among Adolescent Girls of Moroccan Descent in the Netherlands." Identity: An International Journal of Theory and Research 4 (2): 145-69. https://doi.org/10.1207/s1532706xid0402.

Keulen, Ronny, Ton van de Wijngaard, Herman Crompvoets, and Frans Walraven. 2007. Riek van Klank: Inleiding in de Limburgse Dialecten. Sittard: Veldeke Limburg.

King, Cecil. 1977. "Here Come the Anthros." In Indians and Anthropologists: Vine Deloria, Jr, and the Critique of Anthropology, edited by Thomas Biolsi and Larry J. Zimmerman, 115-19. Tucson: University of Arizona Press.

Kitzinger, Celia. 2005. "Heteronormativity in Action: Reproducing the Heterosexual Nuclear Family in After-Hours Medical Calls." Social Problems 52 (4): 477-98. https://doi.org/10.1525/sp.2005.52.4.477.

Koole, Tom, and Mylène Hanson. 2002. 'The Category 'Moroccan' in a MultiEthnic Class." In Language, Interaction and National Identity: Studies in the Social Organisation of National Identity in Talk-in-Interaction, 211-32. Ashgate Publishing.

Koshik, Irene. 2003. "Wh-Questions Used as Challenges." Discourse Studies 5 (1): $51=77$.

Kroon, Sjaak, and Ton Vallen. 2004. Dialect En School in Limburg. Studies in Meertaligheid. Amsterdam: Aksant.

Lamont, Michele, and Virag Molnar. 2002. "The Study of Boundaries in the Social 
Sciences." Annual Review of Sociology 28: 167-95.

Lee, Jung-eun Janie. 2009. “She's Hungarious So She's Mexican But She's Most Likely Indian': Negotiating Ethnic Labels in a California Junior High School." Pragmatics 19 (1):39-63. https://doi.org/10.1075/prag.19.1.03jan.

Lepper, Georgia. 2000. "Analysing Context." In Categories in Text and Talk, 50-61. London: Sage Publications.

Lerner, Gene H. 2019. "When Someone Other than the Addressed Recipient Speaks Next: Three Kinds of Intervening Action After the Selection of Next Speaker." Research on Language and Social Interaction 52 (3). https://doi.org/10.1080/08351813.2019.1657280.

Lerner, Gene H., and Celia Kitzinger. 2007. "Extraction and Aggregation in the Repair of Individual and Collective Self-Reference." Discourse Studies 9 (4): $526-57$.

Lesko, Nancy. 2012. Act Your Age! A Cultural Construction of Adolescence. New York: Routledge.

Leveen, Lois. 1996. "Only When I Laugh: Textual Dynamics of Ethnic Humor." MELUS 21 (4): 29-55.

Liddicoat, Anthony, Susanne Döpke, Kristina Love, and Anne Brown. 1994. "Presenting a Point of View: Caller's Contributions to Talkback Radio in Australia." Journal of Pragmatics 22 (2): 139-56. https://doi.org/10.1016/03782166(94)90064-7.

Makkinga-Clijsen, Jolien. 2017. "Belonging to the Old and Unsuccessfully Aged: Language Practices in a Nursing Home in Maastricht, The Netherlands." Journal of the Anthropological Society of Oxford IX (1): 83-101.

Makoni, Sinfree, and Alastair Pennycook. 2005. "Disinventing and (Re)Constituting Languages." Critical Inquiry in Language Studies: An International Journal 2 (3): 137-56. https://doi.org/10.1207/s15427595cils0203.

Malkki, Liisa. 1992. "National Geographic: The Rooting of Peoples and the Territorialization of National Identity Among Scholars and Refugees." Cultural Anthropology 7 (1): 24-44. https://doi.org/10.1525/can.1992.7.1.02a00030.

Martín Rojo, Luisa. 2008. "Imposing and Resisting Ethnic Categorization in Multicultural Classrooms." In Analysing Identities in Discourse, 31-56. John Benjamins Publishing Company.

Maynard, Douglas W. 2003. Bad Nerws, Good Nerws. Conversational Order in Everyday Talk and Clinical Setting. Chicago \& London: The University of Chicago Press.

Mehan, Hugh, Hubbard Lea, Angela Lintz, and Irene Villanueva. 1994. "Tracking Untracking: The Consequences of Placing Low Track Students in High Track Classes." https://doi.org/10.11436/mssj.15.250.

Mesthrie, Rajend. 2011. "Introduction: The Sociolinguistic Enterprise." In The Cambridge Handbook of Sociolinguistics, edited by Rajend Mesthrie, 1-14. Cambridge: Cambridge University Press.

Moerman, Michael. 1988. Talking Culture: Ethnography and Conversation Analysis. 


\section{Nederlanders and buitenlanders}

Philadelphia: University of Pennsylvania Press.

Møller, Janus Spindler. 2017. "You Black Black': Polycentric Norms for the Use of Terms Associated with Ethnicity.” In Engaging Superdiversity: Recombining Spaces, Times and Language Practices, edited by Karel Arnaut, Martha Sif Karrebæk, Massimiliano Spotti, and Jan Blommaert, 123-46. Bristol: Multilingual Matters.

Møller, Janus Spindler. 2019. "Recognizing Languages, Practicing Languaging.” In Critical Perspectives on Linguistic Fixity and Fluidity: Languagised Lives, edited by Jürgen Jaspers and Lian Malai Madsen, 29-52. New York: Routledge.

Morillo Morales, Gino. 2017. "Es Ich Groter Bin Dan Mag Ik Naar de Basisschool! Analysing the Multingual Landscape Op Eijsden-Margraten's Pre-School Playgrounds.” Master's thesis, Radbout Universiteit Nijmegen, Tilburg University.

Mosher, Rhiannon Michelle. 2015. "Speaking Together: Exploring Discourses of 'Dutchness' in Language Learning, Voluntarism, and Active Citizenship.” $\mathrm{PhD}$ diss., York University. https://yorkspace.library.yorku.ca/xmlui/handle/10315/32084.

Mourabit, Nizar. 2014. "Mensen Die Nederlander Zijn Moeten We Niet Langer Marokkaan Noemen." Het Parool, September 15, 2014. https://www.parool.nl/columns-opinie/mensen-die-nederlander-zijn-moetenwe-niet-langer-marokkaan-noemen be368cef/.

Mourigh, Khalid. 2019. "A Dutch Multiethnolect? Metalinguistic Commentary from Gouda." Applied Linguistics Review 10 (3). https://doi.org/https://doi.org/10.1515/applirev-2017-0053.

Muller, Nick. 2014. "Achter de Schermen Bij Nieuwste Facebook-Hit 'Tattas Be Like." HP/De Tijd, 2014. http://www.hpdetijd.nl/2014-01-08/achter-deschermen-bij-facebook-hit-tattas-like/.

Nørreby, Thomas Rørbeck. 2016. "Ethnic Identifications in Late Modern Copenhagen." In Everyday Languaging: Collaborative Research on the Language Use of Children, edited by Lian Malai Madsen, Martha Sif Karrebæk, and Janus Spindler Møller, 199-218. Berlin/Boston: Walter de Gruyter.

Nørreby, Thomas Rørbeck. 2019. "Ethnic Identities Are Low Social Status Identities." Journal of Pragmatics 140: 127-39. https://doi.org/10.1016/j.pragma.2018.11.018.

Nørreby, Thomas Rørbeck, and Lian Malai Madsen. 2018. "The Symbolic Organization of Languages in a High-Prestige School.” In Critical Perspectives on Linguistic Fixity and Fluidity: Languagised Lives, edited by Jürgen Jaspers and Lian Malai Madsen, 146-64. New York: Routledge.

Nørreby, Thomas Rørbeck, and Janus Spindler Møller. 2015. "Ethnicity and Social Categorization in On- and Offline Interaction among Copenhagen Adolescents." Discourse, Context and Media 8: 46-54. https://doi.org/10.1016/j.dcm.2015.05.006.

Norrick, Neal, and Janine Klein. 2008. "Class Clowns: Talking out of Turn with an Orientation Toward Humor." Lodz Papers in Pragmatics 4 (1): 83-107. https://doi.org/10.2478/v10016-008-0002-6. 
Nortier, Jacomine. 2009. Nederland Meertalenland. Feiten, Perspectieven En Meningen over Meertaligheid. Amsterdam: Aksant.

Nortier, Jacomine, and Margreet Dorleijn. 2008. "A Moroccan Accent in Dutch: A Sociocultural Style Restricted to the Moroccan Community?” International Journal of Bilingualism 12 (1-2): 125-42. https://doi.org/10.1177/13670069080120010801.

O’Reilly, Karen. 2012. Ethnographic Methods. Second edi. Abingdon, Oxon: Routledge.

Omlo, Jurriaan Johannes. 2011. Integratie Én Uit de Gratie? Perspectieven van Marokkaans-Nederlandse Jongrolwassenen. Delft: Uitgeverij Eburon.

Özpamuk, Halil. 2018. "Mijn Stad Is Mijn Hart.” Tegenlicht, NPR. https:/www.vpro.nl/programmas/tegenlicht/kijk/afleveringen/20182019/mijn-stad-is-mijn-hart.html

Papaikonomou, Zoë. 2019. "Tanja Traag van CBS: 'Begrippen "Westers" En "NietWesters” Staan Ter Discussie.” Nieuw Wij, 2019.

https://www.nieuwwij.nl/interview/tanja-traag-cbs-begrippen-westers-enniet-westers-staan-ter-discussie/.

Peräkylä, Anssi. 2007. “Conversation Analysis.” In Qualitative Research Practice, edited by Clive Seale, Giampietro Gobo, Jaber Gubrium, and David Silverman, Paperback, 153-67. London: Sage Publications.

Phalet, Karen, and Marc Swyngedouw. 2002. "National Identities and Representations of Citizenship." Ethnicities 2 (1): 5-30.

Phillips, Anne. 2010. "What's Wrong with Essentialism?” Distinktion 11 (1): 47-60. https://doi.org/10.1080/1600910X.2010.9672755.

Plester, Barbara A., and Janet Sayers. 2007. “Taking the Piss': Functions of Banter in the IT Industry." Humor 20 (2): 157-87. https://doi.org/10.1515/HUMOR.2007.008.

Pollock, Mica. 2004. Colormute: Race Talk Dilemmas in an American School. Princeton and Oxford: Princeton University Press.

Pomerantz, Anita. 1986. "Extreme Case Formulations: A New Way of Legitimating Claims.” Human Studies 9 (2): 219-30.

Pomerantz, Anita, and John Heritage. 2013. "Preference." In The Handbook of Conversation Analysis, edited by Jack Sidnell and Tanya Stivers, 210-28. Malden, MA: Wiley-Blackwell.

Quist, Pia. 2010. "Untying the Language - Body - Place Connection: A Study on Linguistic Variation and Social Style in a Copenhagen Community of Practice." In Language and Space. An International Handbook Of_Linguistic Variation. Volume 1: Theories and Methods, edited by Peter Auer and Jurgen Erich Schmidt, 632-48. Berlin, New York: De Gruyter Mouton.

Rampton, Ben. 2005. Crossing: Language and Ethnicity among Adolescents. Manchester, UK: St. Jerome Publishing.

Rampton, Ben, Karin Tusting, Janet Maybin, Richard Barwell, Angela Creese, and Vally Lytra. 2004. "UK Linguistic Ethnography: A Discussion Paper.” UK Linguistic Ethnography Forum, no. December: 1-24. http://www.uklef.net/publications.html. 


\section{Nederlanders and buitenlanders}

Raymond, Geoffrey. 2003. "Grammar and Social Organization: Yes/No Interrogatives and the Structure of Responding." American Sociological Review 68 (6): 939-67.

Raymond, Geoffrey, and John Heritage. 2006. "The Epistemics of Social Relations: Owning Grandchildren.” Language in Society 35: 677-705. https://doi.org/10.1017/S0047404506060325.

Reekum, Rogier van. 2012. "As Nation, People and Public Collide: Enacting Dutchness in Public Discourse." Nations and Nationalism 18 (4): 583-602. https://doi.org/10.1111/j.1469-8129.2012.00554.x.

Reyes, Angela. 2011. "Racist!': Metapragmatic Regimentation of Racist Discourse by Asian American Youth." Discourse and Society 22 (4): 458-73. https://doi.org/10.1177/0957926510395836.

Rijkschroeff, Rally, Geert Ten Dam, Jan Willem Duyvendak, Marjan de Gruijter, and Trees Pels. 2005. "Educational Policies on Migrants and Minorities in the Netherlands: Success or Failure?” Journal of Education Policy 20 (4): 417-35. https://doi.org/10.1080/02680930500132148.

Robben, Antonius C.G.M. 2012. "Introduction.” In Ethnographic Fieldwork: An Anthropological Reader, edited by Antonius C.G.M. Robben and Jeffrey A. Sluka, Second edi, 513-19. Malden, MA: Wiley-Blackwell.

Robben, Antonius C.G.M., and Jeffrey A. Sluka. 2012. Ethnographic Fieldwork. An Anthropological Reader. Second edi. Malden, MA: Wiley-Blackwell.

Robles, Jessica S. 2019. “Building Up by Tearing Down.” Journal of Language and Social Psychology 38 (1): 85-105. https://doi.org/10.1177/0261927X17744244.

Roggeband, Conny, and Marleen van der Haar. 2018. "Moroccan Youngsters': Category Politics in the Netherlands." International Migration 56 (4): 79-95. https://doi.org/10.1111/imig.12419.

Romaniuk, Tanya. 2013. "Interviewee Laughter and Disaffiliation in Broadcast News Interviews." In Studies of Laughter in Interaction, edited by Phillip Glenn and Elizabeth Holt, 201-20. London: Bloomsbury.

http://www.bloomsbury.com/uk/studies-of-laughter-in-interaction9781441164797/.

Rubin, Beth C. 2006. "Tracking and Detracking: Debates, Evidence, and Best Practices for a Heterogeneous World." Theory into Practice 45 (1): 4-14. https://doi.org/10.1207/s15430421tip4501.

Russell, Lisa. 2005. "It's a Question of Trust: Balancing the Relationship between Students and Teachers in Ethnographic Fieldwork." Qualitative Research 5 (2): 181-95. https://doi.org/10.1177/1468794108099320.

Sabatier, Colette. 2008. "Ethnic and National Identity among Second-Generation Immigrant Adolescents in France: The Role of Social Context and Family." Journal of Adolescence 31 (2): 185-205. https://doi.org/10.1016/j.adolescence.2007.08.001.

Sacks, Harvey. 1995. Lectures on Conversation, Volumes I \& II. Edited by Gail Jefferson. Vol. I, II. Malden, MA: Blackwell Publishing. https://doi.org/10.1002/9781444328301.

Sacks, Harvey, Emanuel A. Schegloff, and Gail Jefferson. 1974. "A Simplest 
Systematics for the Organization of Turn-Taking for Conversation." Language 50: 696-735.

Said, Edward. 1978. Orientalism. London: Routledge \& Kegan Paul.

Schegloff, Emanuel A. 1992. "On Talk and Its Institutional Occasions.” In Talk at Work: Interaction in Institutional Settings, edited by Paul Drew and John Heritage, 101-34. Cambridge: Cambridge University Press.

Schegloff, Emanuel A. 1995. "Introduction." In Lectures on Conversation, Volumes I $E^{2}$ II, edited by Harvey Sacks, ix-1. Malden, MA: Blackwell Publishing.

Schegloff, Emanuel A. 1996. "Confirming Allusions: Toward an Empirical Account of Action.” American Journal of Sociology 102 (1): 161-216.

Schegloff, Emanuel A. 2007. "A Tutorial on Membership Categorization.” Journal of Pragmatics 39 (3): 462-82. https://doi.org/10.1016/j.pragma.2006.07.007.

Schravesande, Freek. 2016. "De Opkomst En Ondergang van de Term 'Allochtoon." NRC, November 1, 2016. https://www.nrc.nl/nieuws/2016/11/01/elke-term-raakt-uiteindelijk-beladen5089788-a1529611.

Schutter, G. de, and B.J.H. Hermans. 2013. “The Limburg Dialects: Grammatical Properties." In Language and Space: An International Handbook of Linguistic Variation. Volume 3: Dutch, edited by F. Hinskens and J. Taeldeman, 356-78. Berlin: Walter de Gruyter.

Seeberg, Marie Louise. 2003. "Dealing with Difference: Two Classrooms, Two Countries, A Comparative Study of Norwegian and Dutch Processes of Alterity and Identity, Drawn from Three Points of View.” Norwegian Social Research NOVA report 18/03.

Shenk, Petra Scott. 2007. “'I'm Mexican, Remember?' Constructing Ethnic Identities via Authenticating Discourse.” Journal of Sociolinguistics 11 (2): 194220. https://doi.org/10.1111/j.1467-9841.2007.00318.x.

Sidnell, Jack. 2008. "Alternate and Complementary Perspectives on Language and Social Life: The Organization of Repair in Two Caribbean Communities." Journal of Sociolinguistics 12 (4): 477-503. https://doi.org/10.1111/j.14679841.2008.00377.x.

Sidnell, Jack. 2010. Conversation Analysis: An Introduction. Malden, MA: WileyBlackwell.

Sidnell, Jack. 2013. "Basic Conversation Analytic Methods." In The Handbook of Conversation Analysis, edited by Jack Sidnell and Tanya Stivers, 77-100. Malden, MA: Wiley-Blackwell.

Sidnell, Jack, and Tanya Stivers. 2013. The Handbook of Conversation Analysis. Edited by Jack Sidnell and Tanya Stivers. Malden, MA: Wiley-Blackwell.

Silva, Emanuel Da. 2015. "Humor (Re)Positioning Ethnolinguistic Ideologies: 'You Tink Is Funny?" Language in Society 44 (2): 187-212. https://doi.org/10.1017/S0047404515000044.

Silverstein, Michael. 2003. "Indexical Order and the Dialectics of Sociolinguistic Life." Language and Communication 23 (3-4): 193-229. https://doi.org/10.1016/S0271-5309(03)00013-2.

Simon, Patrick. 2012. "French National Identity and Integration: Who Belongs to 
the National Community?" Migration Policy Institute May. https://www.migrationpolicy.org/pubs/FrenchIdentity.pdf.

Slik, Frans W.P. van Der, Geert W.J.M. Driessen, and Kees L.J. de Bot. 2006.

"Ethnic and Socioeconomic Class Composition and Language Proficiency: A Longitudinal Multilevel Examination in Dutch Elementary Schools.” European Sociological Review 22 (3): 293-308. https://doi.org/10.1093/esr/jci058.

Slootman, Marieke. 2016. "Substantive Signifiers? Ethnic and Religious Identifications among Second-Generation Immigrants in the Netherlands." Identities: Global Studies in Culture and Power 23 (5): 572-90. https://doi.org/10.1080/1070289X.2015.1035722.

Slootman, Marieke. 2018. Ethnic Identity, Social Mobility and the Role of Soulmates. Amsterdam: Springer. http://www.springer.com/series/13502.

Slootman, Marieke, and Jan Willem Duyvendak. 2015. "Feeling Dutch: The Culturalization of Citizenship and Second-Generation Belonging in the Netherlands." In Fear, Anxiety and National Identity, edited by Nancy Foner and Patrick Simon, 147-68. New York: The Russell Sage Foundation.

Smakman, Dick. 2006. "Standard Dutch in the Netherlands. A Sociolinguistic and Phonetic Description." Utrecht: LOT.

Snel, Erik, Godfried Engbersen, and Arjen Leerkes. 2006. "Transnational Involvement and Social Integration." Global Networks 6 (3): 285-308. https://doi.org/10.1111/j.1471-0374.2006.00145.x.

Speer, Susan A., and Elizabeth Stokoe. 2011. Conversation and Gender. Conversation and Gender. https://doi.org/10.1017/CBO9780511781032.

Spotti, Massimiliano. 2011. "Ideologies of Success for Superdiverse Citizens: The Dutch Testing Regime for Integration and the Online Private Sector."

Diversities 13 (2): 39-52.

Spotti, Massimiliano. 2014a. "The Primary Classroom as a Superdiverse HeteroNormative Space." In Linguistic Superdiversity in Urban Areas: Research Approaches, edited by Ingrid Gogolin and Joana Duarte, 161-78. Amsterdam/Philadelphia: John Benjamins Publishing Company. https://doi.org/10.1075/hsld.2.11spo.

Spotti, Massimiliano. 2014b. "Voices in the Classroom: On Being Caught between Pupils' Inventiveness and Ethnographic Naivety." Ethnography and Education 9 (3): 359-72. https://doi.org/10.1080/17457823.2014.919601.

Spotti, Massimiliano, and Sjaak Kroon. 2009. "Ideologies of Disadvantage: Language Attribution in a Dutch Multicultural Primary School Classroom." JoLIE 2: 179-95. https://doi.org/10.1007/s00180-012-0317-1.

Spradley, James. 1979. The Ethnographic Interview. Long Grove, Illinois: Waveland Press.

Stokoe, Elizabeth. 2012. "Moving Forward with Membership Categorization Analysis: Methods for Systematic Analysis." Discourse Studies 14 (3): 277-303. https://doi.org/10.1177/1461445612441534.

Stokoe, Elizabeth, and Derek Edwards. 2009. "Accomplishing Social Action with Identity Categories: Mediating and Policing Neighbour Disputes.” In 
Theorizing Identities and Social Action, edited by M. Wetherell, 95-115.

Palgrave Macmillan.

Stokoe, Elizabeth, and Janet Smithson. 2001. "Making Gender Relevant:

Conversation Analysis and Gender Categories in Interaction." Discourse \& Society 12 (2): 217-44.

Talmy, Steven. 2009. "Forever FOB? Resisting and Reproducing the Other in High School ESL.” In Beyond Yellow English. Toward a Linguistic Anthropology of Asian Pacific America, 347-65. Oxford University Press.

Tannen, Deborah. 1987. "Repetition in Conversation as Spontaneous Formulaicity." Text E Talk 7 (3): 215-43.

Thijs, Jochem, Saskia Westhof, and Helma Koomen. 2012. "Ethnic Incongruence and the Student-Teacher Relationship: The Perspective of Ethnic Majority Teachers." Journal of School Psychology 50 (2): 257-73. https://doi.org/10.1016/j.jsp.2011.09.004.

Thissen, Lotte. 2013. "The Ambiguities of Limburgerness. Language, Place, and Belonging in Limburg, the Netherlands." Etnofoor, The Netherlands Now 25 (2): 119-43.

Thissen, Lotte. 2018. "Talking in and out of Place: Ethnographic Reflections on Language, Place and (Un)Belonging in Limburg, the Netherlands." PhD diss., Maastricht University. https://doi.org/10.26481/dis.20180111lt.

Thornborrow, Joanna. 2002. Power Talk: Language and Interaction in Institutional Discourse. London and New York: Routledge.

Timmermans, Anneke C., Hans Kuyper, and Greetje van der Werf. 2015. "Accurate, Inaccurate, or Biased Teacher Expectations: Do Dutch Teachers Differ in Their Expectations at the End of Primary Education?" British Journal of Educational Psychology 85 (4): 459-78. https://doi.org/10.1111/bjep.12087.

Verkuyten, Maykel. 2003. "Discourses about Ethnic Group (de-)Essentialism: Oppressive and Progressive Aspects." The British Journal of Social Psychology 42: 371-91. https://doi.org/10.1348/014466603322438215.

Verkuyten, Maykel, and Jochem Thijs. 2010. "Ethnic Minority Labeling, Multiculturalism, and the Attitude of Majority Group Members." Journal of Language and Social Psychology 29 (2008): 467-77. https://doi.org/10.1177/0261927X10377992.

Verkuyten, Maykel, and Ali Aslan Yildiz. 2007. "National (Dis)Identification and Ethnic and Religious Identity: A Study Among Turkish-Dutch Muslims." Personality and Social Psychology Bulletin 33 (10): 1448-62. https://doi.org/10.1177/0146167207304276.

Wagner, Lauren. 2017. Becoming Diasporically Moroccan. Linguistic and Embodied Practices for Negotiating Belonging. Bristol, UK: Multilingual Matters.

Wagner, Lauren B. 2018. "Flirting Diasporically: Visits 'Home' Facilitating Diasporic Encounters and Complex Communities." Journal of Ethnic and Migration Studies 44 (2): 321-40. https://doi.org/10.1080/1369183X.2017.1341716.

Wang, Xuan, Massimiliano Spotti, Kasper Juffermans, Leonie Cornips, Sjaak 
Kroon, and Jan Blommaert. 2014. "Globalization in the Margins: Toward a Re-Evaluation of Language and Mobility." Applied Linguistics Review 5 (1): 23-44. https://doi.org/10.1515/applirev-2014-0002.

Weerd, Pomme van de. 2019. “'Those Foreigners Ruin Everything Here': Interactional Functions of Ethnic Labelling among Pupils in the Netherlands." Journal of Sociolinguistics 23 (3): 244-62. https://doi.org/10.1111/josl.12344.

Weerd, Pomme van de. 2020. "Categorization in the classroom: a comparison of teachers' and students' use of ethnic categories." Journal of Multicultural Discourses. Advance online publication. https://doi.org/10.1080/17447143.2020.1780243

Weerd, Pomme van de. Forthcoming. "Ethnic labeling among pupils with migration backgrounds: 'Turks', 'Moroccans', and 'foreigners' in the Netherlands." Dutch Journal of Applied Linguistics.

Weiner, Melissa F. 2016. "Racialized Classroom Practices in a Diverse Amsterdam Primary School: The Silencing, Disparagement, and Discipline of Students of Color." Race Ethnicity and Education 19 (6): 1351-67. https://doi.org/10.1080/13613324.2016.1195352.

Welle, Inge van der. 2011. "Flexibele Burgers? Amsterdamse Jongvolwassenen over Lokale En Nationale Identiteiten.” PhD diss., University of Amsterdam. https://dare.uva.nl/search?identifier=e5175c19-e119-4fa0-8e1088070e16a298

Whitehead, Kevin A. 2012. "Racial Categories as Resources and Constraints in Everyday Interactions: Implications for Racialism and Non-Racialism in PostApartheid South Africa." Ethnic and Racial Studies 35 (7): 1248-65. https://doi.org/10.1080/01419870.2011.591407.

Whitehead, Kevin A. 2018. "Managing the Moral Accountability of Stereotyping." Journal of Language and Social Psychology, $0261927 X 1772367$. https://doi.org/10.1177/0261927x17723679.

Whitehead, Kevin A., Brett Bowman, and Geoffrey Raymond. 2018. "Risk Factors' in Action: The Situated Constitution of 'Risk' in Violent Interactions." Psychology of Violence 8 (3): 329-38. https://doi.org/10.1037/vio0000182.

Whitehead, Kevin A., and Gene H. Lerner. 2009. "When Are Persons 'White'?: On Some Practical Asymmetries of Racial Reference in Talk-in-Interaction." Discourse and Society 20 (5): 613-41. https://doi.org/10.1177/0957926509106413.

Willis, Paul. 1981. Learning to Labor. How Working Class Kids Get Working Class Jobs. New York: Columbia University Press.

Willis, Paul, and Mats Trondman. 2000. "Manifesto for Ethnography." Ethnography 1 (1): 5-16.

Wimmer, Andreas. 2013. Ethnic Boundary Making. Institutions, Power, Networks. Oxford: Oxford University Press.

Winkler Reid, Sarah. 2015. "Making Fun out of Difference: Ethnicity-Race and Humour in a London School." Ethnos 80 (1): 23-44. https://doi.org/10.1080/00141844.2013.801504. 
Wooffit, Robin. 2005. Discourse Analysis and Conversation Analysis. A Comparative and Critical Introduction. London: Sage Publications.

Woolard, Kathryn. 2018. "Playing against Peripheralization: A Commentary." In The Sociolinguistics of Place and Belonging. Perspectives from the Margins, edited by Leonie Cornips and Vincent de Rooij, 115-124. John Benjamins Publishing Company.

Woolard, Kathryn A. 2008. "Why Dat Now?: Linguistic-Anthropological Contributions to the Explanation of Sociolinguistic Icons and Change." Journal of Sociolinguistics 12 (4): 432-52. https://doi.org/10.1111/j.14679841.2008.00375.x.

Yanow, Dvora, and Marleen van der Haar. 2013. "People out of Place: Allochthony and Autochthony in the Netherlands' Identity Discourse-Metaphors and Categories in Action." Journal of International Relations and Development 16 (2): 227-61. https://doi.org/10.1057/jird.2012.13.

Zimmerman, Don H., and Melvin Pollner. 1970. "The Everyday World as a Phenomenon*." In Understanding Everyday Life: Toward the Reconstruction of Sociological Knowledge, edited by Jack D. Douglas, 80-104. London: Routledge \& Kegan Paul. https://doi.org/10.4324/9781351327329-4. 
224 Nederlanders and buitenlanders 


\section{Nederlandse samenvatting}

In Nederlanders en Buitenlanders onderzoek ik hoe leerlingen van een middelbare schoolklas in Venlo, 'klas 3/4b,' zich verhielden tot lokale en maatschappelijke sociale hiërarchieën, en die onderhandelden, door zichzelf en elkaar te categoriseren in etnische termen en door het gebruiken van verschillende talige middelen. De onderzoeksvraag, geïntroduceerd in Hoofdstuk 1, is: Wat zijn de betekenissen en functies van etnische categorieën en talige middelen voor de leerlingen en docenten van klas $3 / 4 \mathrm{~b}$ ? Ik heb dit onderzocht op basis van data verzameld tijdens negen maanden etnografisch veldwerk met de leerlingen, en door het analyseren van interacties tussen leerlingen, docenten, en mijzelf, voornamelijk met Membership Categorization Analysis (MCA) en Conversatie Analyse (CA).

Ongeveer de helft van de leerlingen van klas $3 / 4 b$ had een migratieachtergrond, en hoewel zij in Nederland waren geboren, categoriseerden zij zichzelf en elkaar regelmatig met de labels 'buitenlander,' 'Marokkaan' en 'Turk,' en refereerden zij naar anderen (maar niet zichzelf) als 'Nederlander.' Deze categorisatie maakte deel uit van alledaagse interacties, terwijl ze elkaar plaagden, schoolopdrachten maakten, of roddelden over bekenden. Ook het gebruik van verschillende talige middelen (naast Standaardnederlands gebruikten de leerlingen in hun interacties onder andere Arabisch, Berbers, Turks, Venloos, en Tegels) bleek van belang te zijn in het vormgeven en onderhandelen van deze categorieën.

In veel maatschappelijk, en ook academisch, discours in Nederland wordt zelf-categorisatie met andere labels dan 'Nederlander' geïnterpreteerd als uiting van een gebrek aan 'integratie' of 'jezelf thuis voelen' in Nederland. Daarbij denkt men regelmatig dat wie zichzelf (bijvoorbeeld) 'Marokkaan' noemt een emotionele verbinding heeft met Marokko, of een bepaalde socioculturele of religieuze oriëntatie. In het proefschrift stel ik dat de precieze en vaak meervoudige betekenis van etnische labels daarmee wordt onderschat, en dat het nodig is om in acht te nemen hoe mensen zelf, in hun eigen interacties, die categorieën gebruiken. Het bewijs daarvoor vond ik door negen maanden te spenderen met de leerlingen van klas $3 / 4 b$, een vmbo-basis en kader klas, waar ik data verzamelde door middel van participerende observatie: ik ging meerdere dagen per week met de leerlingen mee naar de lessen, soms hielp ik ze met opdrachten, en ik had veel gesprekken met leerlingen en docenten. Terwijl ik op school was droeg ik een opnameapparaat (enkel audio), waardoor ik interessante of opvallende interacties later terug kon 
beluisteren, in al hun details kon transcriberen, en op die manier analyseren. Met deze sociolinguïstisch-etnografische aanpak heb ik in het proefschrift laten zien dat etnische categorieën niet hetzelfde betekenen voor iedereen, noch in iedere context. Bovendien stel ik dat etnische categorisatie niet slechts gezien moet worden als een manier waarop participanten informatie over zichzelf of hun 'identiteit' verstrekken, maar dat het ook een daad in interactie kan zijn: door zichzelf te categoriseren, konden leerlingen bijvoorbeeld machtsposities in interactie verschuiven, en met humor over categorieën haalden zij de angel uit potentieel gevoelige interacties. Op basis van nauwkeurige analyses van lokale interacties, demonstreer ik in dit proefschrift i) dat leerlingen uit klas 3/4b meerdere betekenissen gaven aan nationale en etnische categorieën zoals 'Marokkaan,' 'Turk,' 'Nederlander' en 'buitenlander,' ii) dat ze die categorieën en bepaalde talige middelen gebruikten om er lokale hiërarchieën mee op te zetten, te handhaven en onderhandelen, iii) dat ze die categorieën bovendien gebruikten om er interactionale behoeften mee op te lossen, en iv) dat leerlingen en docenten niet slechts lokale hiërarchieën onderhandelen, maar ook de confrontatie aangingen met wijdverspreide definities van wat Marokkaan, Turk of Nederlander betekenen, en met hoe die labels gewoonlijk gerangschikt zijn.

Ik begin deze samenvatting met een korte uitleg van de inhoud van ieder empirisch hoofdstuk, waarna ik de bijdragen van het proefschrift zal uitlichten.

\section{Samenvatting van de empirische hoofdstukken}

In Hoofdstuk 3 analyseer ik het complexe spectrum aan betekenissen van de categorieën 'Marokkaan,' 'Turk,' 'Nederlander' en 'buitenlander' onder de leerlingen van klas 3/4b. Deze categorieën dragen een geschiedenis aan betekenissen bij zich, die in deze klas werden onderhandeld, betwist, versterkt, en aangepast. Het was voor de leerlingen vanzelfsprekend dat iedereen tenminste in één van de hiervoor genoemde categorieën viel. De gevolgen van (bijvoorbeeld) het zijn van 'Turk' of 'Nederlander' waren echter minder eenduidig en werden regelmatig onderhandeld. Leerlingen verbonden vaak factoren als religie, uiterlijk, stijl, of gedrag, aan categorieën, hoewel ze niet allemaal hetzelfde dachten over de manieren waarop die relaties werden uitgedrukt, en hoe essentieel ze waren voor een categorie. Echter, in tegenstelling tot de suggestie dat jezelf 'Marokkaan,' 'Turk' of 'buitenlander' noemen een uiting is van een gebrek aan integratie in, of emotionele verbondenheid met, Nederland, hadden leerlingen zelf het zelden over deze onderwerpen wanneer zij spraken over 'Marokkanen,' 'Turken,' of 'buitenlanders.' Ik wijs er in dit hoofdstuk dus op dat zelf-categorisatie als 
'Marokkaan,' 'Turk' of 'buitenlander' niet vanzelfsprekend gezien kan worden als een teken van een gebrek aan 'jezelf thuis voelen' in, of emotionele verbondenheid voelen met Nederland, noch dat het wijst op een verbondenheid met een ander land (zoals in de literatuur en het maatschappelijk debat soms wordt gesuggereerd). Onder de leerlingen van klas 3/4b dienden deze labels vaak om lokale sociale hiërarchieën op te zetten. Opvallend was dat categorieën die in de Nederlandse samenleving vaak gestigmatiseerd zijn - bijvoorbeeld 'Marokkaan' - onder de leerlingen van deze school juist prestigieus waren. Het was 'cool' om 'Marokkaan,' 'buitenlander,' of 'Turk' te zijn, en de categorie 'Nederlander' werd juist verbonden aan lokaal ongewilde eigenschappen zoals 'braaf zijn. Ik suggereer in dit hoofdstuk dat die omkering van de gewoonlijke hiërarchie onder deze categorieën (waarin 'Nederlander' juist vaak als meer prestigieus gezien wordt) gezien kan worden als een strategie waarmee deze leerlingen hun gemarginaliseerde positie in de Nederlandse maatschappij enigszins verzachtten. Door hun migratieachtergrond en hun positie aan de bodem van de hiërarchie van het Nederlandse onderwijssysteem (als leerlingen van vmbobasis en kader) hadden zij te maken met lidmaatschap van ten minste twee gestigmatiseerde categorieën. Door het veranderen van de associaties van één van die categorieën maakten deze leerlingen, in hun lokale context en onder hun medeleerlingen, aanspraak op een aanzien dat ze buiten de school vaak ontbeerden.

In Hoofdstuk 4 stel ik dat categorieën niet slechts geanalyseerd kunnen worden als een indicatie van 'wie iemand is,' maar ook als daden in interactie. Door middel van Conversatie Analyse (CA) en Membership Categorisation Analysis (MCA), analyseer ik wat leerlingen bereikten in interacties door de categorieën 'Marokkaan,' 'Turk,' 'Nederlander' en 'buitenlander' te noemen. Doordat categorieën in deze context verbonden werden aan allerlei eigenschappen en associaties (bijvoorbeeld: 'Nederlanders en buitenlanders hebben verschillende gebruiken'; of 'Marokkanen worden gediscrimineerd'), riep het noemen van een categorie zo'n associatie op. Door het noemen van labels konden leerlingen bijvoorbeeld machtsposities binnen een interactie verschuiven (bijvoorbeeld van 'ondergeschikte leerling' naar 'expert op het gebied van Turkse gebruiken'), of konden ze medeleerlingen plagen en daarmee de angel uit potentieel gevoelige interacties halen. Met name humor rondom categorieën was gebruikelijk onder de leerlingen van klas 3/4b. Leerlingen met een migratieachtergrond beledigden regelmatig de categorie waarvan zij zelf, of hun conversatiepartner, deel uitmaakten, op manieren die sterk leken op een wijdverspreid xenofobisch discours. Op die manier leken leerlingen aan de hand van die beledigingen zich ook het xenofobe maatschappelijk discours eigen te maken, en daarmee minder kwetsend of 
bedreigend. Maar niet elke leerling kon vrij gebruik maken van de categorieën 'Turk,' 'Marokkaan,' of 'buitenlander,' zeker niet als zij als 'Nederlander' gecategoriseerd waren. Als zij bijvoorbeeld de categorieën 'Marokkaan' of 'Turk' gebruikten om anderen te plagen, kon dit gezien worden als een echo van het maatschappelijke discours. Op die momenten bleek dat categorieën niet slechts lokale, vernieuwende betekenissen hadden, maar dat hun stigmatiserende betekenis ook terug naar voren kon komen. In dit hoofdstuk concludeer ik dat, hoewel categorieën lokale betekenissen en functies kunnen hebben, en niet altijd dienen om een 'identiteit' te profileren, het intensieve gebruik ervan ook leidt tot de versterking van het idee dat deze verschillende categorieën een objectieve waarheid vertegenwoordigen.

In Hoofdstuk 5 behandel ik de rol van categorisatie in interacties met docenten. Dit hoofdstuk laat zien dat leerlingen niet de enigen waren die categoriseerden op de school waar ik mijn onderzoek deed: docenten onderhandelden evengoed over de betekenissen en functies van categorieën. Soms problematiseerden docenten de zelf-categorisatie van leerlingen als 'buitenlander,' 'Turk,' of 'Marokkaan': ze warden dan bezorgd of leerlingen zich wel thuis voelden op school, of corrigeerden leerlingen door te zeggen dat ze "geen buitenlanders, maar Nederlanders" waren. Dit leek gemotiveerd te zijn door hoe het gebruik van die labels gewoonlijk geïnterpreteerd wordt, namelijk dat wie zichzelf als niet-Nederlands voorstelt een gebrek aan integratie vertoont of zich niet thuis voelt in Nederland. Terwijl leerlingen schakelden tussen het gebruik van categorieën met deze conventionele associaties, aan de ene kant, en categorieën met hun lokale associaties (zoals voornamelijk geanalyseerd in Hoofdstuk 3) aan de andere kant, leken docenten categorieën dus vooral op een conventionele manier te interpreteren. In sommige interacties konden de reacties van docenten op zelf-categorisaties van leerlingen ook begrepen worden als een daad in interactie, net zoals de categorisaties van leerlingen zelf. In die zin kon het betwisten van een zelfcategorisatie als 'buitenlander' ook dienen om autoriteit te benadrukken, orde te herstellen, of een leerling te disciplineren. Hoewel docenten lieten merken dat ze graag wilden dat hun leerlingen zichzelf als 'Nederlander' categoriseerden, maakten zij op andere momenten een duidelijk onderscheid tussen leerlingen met en zonder migratieachtergrond. Ze leken op die manier te worstelen met het contrast tussen hun wens dat leerlingen zichzelf als 'Nederlander' zagen, en hun overtuiging dat er duidelijke verschillen waren leerlingen met en zonder migratieachtergrond, verschillen die bovendien werden benadrukt door de leerlingen zelf. Ik eindig dit hoofdstuk door te benadrukken dat het niet per definitie slecht of onverantwoordelijk is voor docenten om dezelfde labels te gebruiken als hun leerlingen. Sommige docenten gebruikten labels op een manier die sterk leek op de manier waarop 
leerlingen dat zelf deden, bijvoorbeeld in een plagende interactie, en dit kan juist werken om de relatie tussen leerling en docent te verbeteren. Echter, als docenten in deze context inclusie willen bevorderen, lijkt het onwaarschijnlijk dat dit te realiseren valt door zelf-categorisaties als 'Marokkaan,' 'Turk' of 'buitenlander' te problematiseren, of door leerlingen te stimuleren om zichzelf 'Nederlander' te noemen. Het label 'Nederlander' heeft voor leerlingen immers een onwenselijke betekenis, omdat ze door 'Nederlanders' vaak als niet-Nederlands worden gezien, en omdat deze categorie hier, in deze lokale context, ook met niet-coole, te schoolse, betekenissen wordt geassocieerd. Het bevorderen van een gevoel van inclusie zou dan beter kunnen werken door andere categorieën te gebruiken zonder de verscheidenheid aan complexe lokale en conventionele betekenissen van een label als 'Nederlander.'

Als de symbolische organisatie van categorieën, beschreven in Hoofdstukken 3 tot en met 5 , één van de manieren was waarop leerlingen de confrontatie aangingen met lokale en wijder verspreide sociale hiërarchieën, zo beschreef Hoofdstuk 6 hoe meertaligheid en de symbolische organisatie van talige middelen nog zo'n manier was. Hoewel de school in Venlo geen expliciet taalbeleid had, vonden docenten het vanzelfsprekend dat iedereen Standaardnederlands moest spreken in de les. Dit veroorzaakte fricties, aangezien de leerlingen over een grote variëteit aan talige competenties beschikten: naast Standaardnederlands ${ }^{36}$ (wat docenten en leerlingen het meest gebruikten in hun communicatie op school) spraken sommige leerlingen ook bijvoorbeeld Limburgs, Marokkaans Arabisch, Berbers, of Turks, en gebruikten zij al deze talen in hun dagelijkse interacties op school. De betekenis van het gebruik van die talige middelen verschilde: onder de leerlingen, en op school, was het prestigieus om uitdrukkingen in het Marokkaans Arabisch, Turks, of Berbers te gebruiken, maar niet zozeer om Limburgs te spreken. Op die manier weerspiegelde de symbolische organisatie van talen onder de leerlingen dus de patronen van de hiërarchisering van categorieën onder leerlingen zoals die in Hoofdstukken 3 tot 5 verkend werden. De symbolische organisatie van diezelfde talen volgde in de uitingen van docenten een tegenovergesteld patroon. $\mathrm{Zij}$ handhaafden het Standaardnederlandse taalbeleid met name wanneer leerlingen Arabisch,

${ }^{36}$ Nederlands zoals het gesproken word door inwoners van Limburg, en door de leerlingen in deze studie, wordt niet altijd als 'standaard' gezien door sprekers uit andere regio's. Ik gebruik echter toch de term 'Standaardnederlands' om te refereren naar het Nederlands zoals het gesproken werd door de leerlingen en docenten in deze studie, omdat deze sprekers het zelf op deze manier zagen, en om het verschil te benadrukken tussen het gebruik van (wat gepercipieerd werd als) 'Nederlands' en 'dialect.' 
Berbers, of Turks spraken, en niet wanneer zij Venloos of Tegels dialect spraken, en verantwoordden dit door te bouwen op ideologieën van talen en sprekers als verbonden aan bepaalde plaatsen. Limburgs werd als acceptabel gezien omdat de school per slot van rekening in Limburg lag, terwijl Turks, bijvoorbeeld, werd voorgesteld als een vreemde, en daarom ongepaste taal in deze context. In het hoofdstuk suggereerde ik dat het insisteren van docenten op Standaardnederlands als leerlingen Turks, Arabisch of Berbers spraken, en het ongemoeid laten van leerlingen die Limburgs spraken, wijst op veranderde en veranderende zorgen rondom taalgebruik in Nederland. Waar het gebruik van dialect in Limburg vroeger werd gezien als 'onbeschaafd' en geassocieerd werd met (onderwijs)achterstand, lijkt dit stigma grotendeels te zijn verdwenen, op zijn minst in deze context (hoewel dialecten nog steeds vooral in de informele sfeer voorkomen). Zorgen over taalgebruik als een teken van 'beschaving' zijn verschoven naar zorgen over taal als een embleem van 'thuishoren.' De zorg van docenten over het zich al dan niet 'thuisvoelen' van hun leerlingen was herkenbaar in de reacties van docenten op de zelfcategorisaties van hun leerlingen, net als in hun reacties hun gebruik van andere talen dan Nederlands. Dit demonstreert hoe de symbolische organisatie van talen steeds veranderende maatschappelijke zorgen en patronen van sociale stratificatie reproduceert.

\section{Wetenschappelijke en maatschappelijke bijdrages}

Ik licht hier graag vier bijdragen uit van dit proefschrift aan de wetenschap en de maatschappij.

Ten eerste hoop ik te hebben laten zien dat de betekenissen en functies van etnische categorieën niet voor lief genomen kunnen worden, maar dat ze in hun context moeten worden bestudeerd. Ik heb bijgedragen aan een dieper begrip van etnische categorisatie door de lokale betekenissen en interactionele functies hiervan te analyseren, en daarbij in acht te nemen hoe deze lokale betekenissen en functies in verband staan met discoursen die in Nederland op een bredere, maatschappelijke schaal voorkomen. In verschillende landen met een vergelijkbare immigratiegeschiedenissen is geobserveerd dat migranten en hun nageslacht zichzelf categoriseren aan de hand van het land van afkomst, in plaats van het land van verblijf (bijvoorbeeld in Duitsland, zie Bozay 2012, in Frankrijk, zie Simon 2012, en in België, zie Jaspers 2011a). Hoewel veel sociolinguïstisch en antropologisch werk heeft benadrukt dat etnische identiteiten contextspecifiek, intersubjectief, en discursief vormgegeven zijn, en onderhandeld worden in interactie (zie bijv. Androutsopoulos en Georgakopoulou 2003; Jaspers 2005b; Jørgensen 2005; Rampton 2005), wordt zelf-categorisatie aan de hand van het land van herkomst nog steeds 
vaak geïnterpreteerd als een gebrek aan identificatie met het land van verblijf (zie bijv. Agirdag, van Houtte, en van Avermaet 2011; Ersanilli en Koopmans 2010; Huijnk en Dagevos 2012; Phalet en Swyngedouw 2002; Verkuyten en Yildiz 2007). Een belangrijke boodschap van dit proefschrift is dat, om te weten wat etnische labels betekenen en waarom mensen ze gebruiken, het nodig is om interactionele data te bestuderen in een context die de onderzoeker goed kent; met andere woorden, om een sociolinguïstischetnografische benadering te nemen waarbij zowel lokale omstandigheden als processen op grotere schaal in acht worden genomen. Met deze benadering heb ik laten zien dat zelf-categorisatie met de labels 'Marokkaan,' 'Turk' of 'buitenlander' niet per se een gebrek aan 'thuis voelen' of verbondenheid met Nederland signaleren, of de aanwezigheid van verbondenheid met een ander land. De participanten van deze studie gebruikten categorieën onder andere om lokale sociale hiërarchieën vorm te geven. Bovendien heeft dit proefschrift gedemonstreerd dat er ook verder gekeken kan worden dan het concept 'identiteit' in de analyse van categorisatie in etnische termen. Zelfs als men 'etnische' termen gebruikt, verwijzen sprekers niet eenvoudig naar hun etnische identiteit. Participanten van dit onderzoek deden ook dingen door het gebruik van labels: zij spraken anderen tegen, verschoven institutionele machtsposities, of plaagden elkaar. Door de interactionele functies van etnische categorieën te benadrukken hoop ik een 'fetishizering van etniciteit' (Rampton 2005) tegen te gaan, ofwel de neiging om individuen als 'exotisch' en 'anders' te bestempelen en 'etnische identiteit' te lezen in alledaagse acties.

Ten tweede heb ik laten zien dat de combinatie van etnografische methodes voor het verzamelen van data, en etnomethodologische methodes voor het analyseren van die data, geschikt zijn om zowel een tot platitude gereduceerd constructivisme, alsmede de neiging tot generaliseringen, te vermijden die met name Brubaker $(2002,2004)$ signaleert in de studie van etnische identificatie. Brubaker stelt dat onderzoekers vaak claimen dat hun benadering van etniciteit 'constructief is, maar dat zij in hun analyses tegelijkertijd vaak het bestaan van 'etnische groepen' aannemen. Mijn doel in dit proefschrift was om zulke generaliseringen te vermijden door etnische categorieën als het object van analyse te behandelen in plaats van als instrument van analyse, en daarmee het hardnekkige idee van etniciteit te verklaren in de levens van participanten zonder etniciteit als objectieve waarheid te presenteren. Ik heb dit gedaan door etnografie te combineren met Conversatie Analyse (CA) en Membership Categorization Analysis (MCA). Aan de ene kant versterkt de etnografische basis van het proefschrift de analyses van interacties, onder andere doordat de meeste interacties die ik analyseer gekozen zijn als voorbeelden van gebruikelijke interacties tussen participanten. Zonder de langdurige betrokkenheid van etnografisch veldwerk had ik 
gebruikelijke en ongebruikelijke interacties niet van elkaar kunnen onderscheiden, en zou ik niet hebben kunnen beargumenteren welke interactionele functies categorisaties hadden in deze context. Verder heeft etnografie me geholpen om de gesitueerdheid van de interacties te verkennen, en te erkennen dat elk taalgebruik een historische, sociale, en culturele context heeft. De nadruk op reflexiviteit in etnografie heeft het bovendien mogelijk gemaakt om de invloed te onderzoeken van mijn aanwezigheid op de context en interacties die ik analyseer heeft. Het gebruik van CA en MCA heeft de etnografische kant van dit proefschrift versterkt door de karakteristieke systematische en grondige methodes van analyse die deze methodes vereisen. Met het gedetailleerde transcriptie-systeem van CA en MCA was het mogelijk om mijn data en argumenten te toetsen met andere onderzoekers, en dat maakt het mogelijk voor anderen om deze data te herzien en mijn analyses te betwisten. Bovenal heeft het gebruik van CA en MCA mij gestimuleerd om me te richten op wat mijn participanten doen in interacties, waardoor ik voorbij het 'etnische' van 'etnische' labels kon kijken.

De derde bijdrage van dit proefschrift is dat het heeft laten zien dat manifestaties van 'diversiteit' (met name in etniciteit en taal) als gevolg van globalisering ook bestudeerd moeten worden op plekken die als perifeer worden gezien. Veel onderzoek naar de effecten van globalisering heeft zich geconcentreerd op plekken waar de gevolgen hiervan duidelijk zichtbaar zijn, met name in grote steden met veel diversiteit in mensen en talen (Wang et al. 2014). Cornips en de Rooij (2018b) stellen dat die concentratie onterecht is omdat globalisering zich ook duidelijk voordoet in wat men als perifere gebieden beschouwt. Dit proefschrift draagt bij in het corrigeren van deze tendens door de discursieve manifestatie van globalisering (het gebruik van etnische labels), te onderzoeken in de vanuit een nationaal oogpunt perifere stad Venlo. 28.4 Procent van de inwoners van Venlo heeft een migratieachtergrond, in vergelijking met 23.6 procent in Nederland (Centraal Bureau voor de Statistiek 2019a). In klas 3/4b had ongeveer de helft van de leerlingen een migratieachtergrond, en zij verwezen dagelijks naar deze achtergrond. De tekenen van globalisering waren in deze context dus onmiskenbaar. De manier waarop dit uiting vond in de zelf-categorisatie van leerlingen leek weliswaar beïnvloed te zijn door deze specifieke omgeving. Waar men in steden als Amsterdam en Rotterdam namelijk heeft opgemerkt dat veel mensen met een migratieachtergrond zichzelf 'Amsterdammer' of 'Rotterdammer' noemen in plaats van 'Nederlander' (Omlo 2011; van der Welle 2011; Özpamuk 2018), zag ik de leerlingen van klas 3/4b zich nooit 'Venlonaar' of 'Limburger' noemen. Dit zou te maken kunnen hebben met de specifieke eigenschappen van Venlo en Limburg. Dat men Limburg vaak associeert met een sterke lokale identiteit en specifieke socioculturele en talige 
eigenschappen, kan de labels 'Limburger' of 'Venlonaar' minder toegankelijk maken voor wie men gewoonlijk als 'divers' ziet.

De vierde bijdrage van dit proefschrift is dat, waar veel studies zich óf op het gebruik van zogenaamde 'immigrantentalen,' of op het gebruik van dialect in de klas richten, ik in dit proefschrift in beperkte mate beide met elkaar vergeleken heb. Hiermee heb ik de symbolische organisatie van talen en hun sprekers in klas 3/4b geanalyseerd, en heb ik het aangetoond hoe deze symbolische organisatie bredere patronen van sociale stratificatie reflecteert en reproduceert. De vergelijking van dialecten en 'immigrantentalen' liet zien dat, hoewel men het Limburgs nog steeds vooral in de informele sfeer acceptabel vindt, en het dus geen officiële positie in het onderwijs heeft, er niet meer zo'n sterk stigma op rust als eerst het geval was. Zorgen over taalgebruik als teken van 'beschaving,' die aan de basis stonden van het stigma op Limburgs, zijn verschoven naar zorgen over taal en 'thuishoren.' Aan de basis hiervan staan taalideologieën zoals het idee dat er onlosmakelijke banden bestaan tussen mensen, talen, en plekken, waardoor men van bepaalde talen en hun sprekers vindt dat ze er wel of niet 'bij horen' (Thissen 2018). In een sociopolitiek klimaat waarin mensen met een migratieachtergrond veel geproblematiseerd worden, zijn het de talen die met die mensen worden geassocieerd die nu de zorgen en afkeuring krijgen die de dialecten ooit te beurt vielen. De patronen van handhaving van het Standaardnederlands taalbeleid op school - waar, over het algemeen, docenten het beleid veel strenger handhaafden wanneer leerlingen Turks, Arabisch, of Berbers spraken, dan wanneer ze dialect spraken - reflecteerde huidige maatschappelijke zorgen in Nederland, en met name de vraag die men in veel maatschappelijk en politiek debat stelt over de mate waarin mensen met een migratieachtergrond zich (genoeg) thuis voelen in Nederland (Duyvendak, Reinders, and Wekker 2016). 
234 Nederlanders and buitenlanders 


\section{Résumé français}

Dans Néerlandais et étrangers, j'étudie la façon dont les élèves du secondaire à Venlo, 'classe 3/4b,' se sont référés aux hiérarchies sociales locales et sociétales, et comment ils ont traité ce sujet, en se catégorisant eux-mêmes et les uns les autres en termes ethniques et en utilisant différents moyens linguistiques. La question de recherche, introduite dans le Chapitre 1, est la suivante: Quelles sont les significations et les fonctions respectives des catégories ethniques et des moyens linguistiques utilisés pour les élèves et les enseignants de la classe 3/4b ? J'ai mené cette étude sur la base des données recueillies pendant neuf mois de travail ethnographique sur le terrain avec les élèves, et en analysant les interactions entre les élèves, les enseignants et moi-même, principalement avec l'analyse de la catégorisation des membres (ACM) et l'analyse de la conversation (AC).

À peu près la moitié des élèves de la classe $3 / 4 \mathrm{~b}$ sont d'origine étrangère et, bien qu'ils soient nés aux Pays-Bas, ils se classent régulièrement, euxmêmes et les autres, sous les étiquettes 'étranger', 'Marocain' et 'Turc', et qualifient les autres (mais pas eux-mêmes) de 'Néerlandais'. Cette catégorisation faisait partie des interactions quotidiennes, que ce soit en se taquinant, en faisant ses devoirs ou en racontant des ragots sur des connaissances. L'utilisation de divers moyens linguistiques (en plus du néerlandais standard, les élèves ont utilisé l'arabe, le berbère, le turc, et les dialectes régionaux de Venlo et Tegelen, entre autres, dans leurs interactions) s'est également avérée importante pour élaborer ces catégories et en discuter.

Dans de nombreux discours sociaux mais aussi universitaires aux PaysBas, l'auto-catégorisation avec des étiquettes autres que 'Néerlandais' est interprétée comme étant l'expression d'un manque d'intégration' ou de sentiment d'être 'chez soi' aux Pays-Bas. En outre, les gens pensent souvent que ceux qui se disent (par exemple) 'Marocains' ont un lien émotionnel avec le Maroc, ou une certaine orientation socioculturelle ou religieuse. Dans ma thèse, je défends l'idée que cela sous-estime la signification précise et souvent multiple des étiquettes ethniques, et qu'il est donc nécessaire de considérer comment les gens eux-mêmes, dans leurs propres interactions, utilisent ces catégories. J'en ai trouvé la preuve en passant neuf mois avec les élèves de la classe 3/4b, une classe dans l'enseignement professionnel, pendant lesquels j'ai collecté des données par le biais de l'observation participative : j'ai participé aux cours avec les élèves plusieurs jours par semaine, parfois en les aidant à faire des devoirs, et j'ai eu de nombreuses conversations avec les élèves et les 
enseignants. Lorsque j'étais à l'école, je portais un appareil d'enregistrement (audio uniquement), ce qui me permettait de réécouter par la suite des interactions intéressantes ou frappantes, de les transcrire en détail et ainsi de les analyser. Grâce à cette approche sociolinguistique et ethnographique, j'ai pu démontrer dans ma thèse que les catégories ethniques n'ont pas la même signification pour tous, ni dans tous les contextes. De plus, je soutiens que la catégorisation ethnique ne doit pas être considérée uniquement comme un moyen de fournir des informations sur soi-même ou sur son 'identité, mais qu'elle peut aussi être un acte d'interaction : par exemple, en se catégorisant, les élèves peuvent changer de position de pouvoir dans l'interaction, et en faisant preuve d'humour à propos des catégories, ils arrivent à désamorcer le sujet dans des interactions potentiellement sensibles ou délicates. Sur la base d'analyses précises des interactions locales, je démontre dans cette thèse i) que les élèves de la classe $3 / 4 \mathrm{~b}$ ont donné des significations multiples aux catégories nationales et ethniques telles que 'Marocain', 'Turc', 'Néerlandais' et 'étranger', ii) qu'ils ont utilisé ces catégories et certains moyens linguistiques pour définir et établir avec elles des hiérarchies locales, et d'en discuter, iii) qu'ils ont également utilisé ces catégories pour répondre à des besoins d'interaction, et iv) que les élèves et les enseignants ne se contentent pas de débattre des hiérarchies locales, mais qu'ils sont également confrontés à des définitions très répandues de ce que signifient les termes 'Marocain', 'Turc' ou 'Néerlandais', et à la manière dont ces étiquettes sont généralement classées.

Je commencerai ce résumé par une brève explication du contenu de chaque chapitre empirique, après quoi je soulignerai les éléments apportés par la thèse.

\section{Résumé des chapitres empiriques}

Dans le Chapitre 3, j'analyse le spectre complexe des significations des catégories 'Marocain', 'Turc', 'Néerlandais' et 'étranger' parmi les élèves de la classe $3 / 4 b$. Ces différentes catégories ont un passé de significations qui ont été discutées, contestées, renforcées et adaptées au sein de cette classe. Pour les élèves, il était évident que chacun d'entre eux appartenait à au moins une des catégories mentionnées ci-dessus. Cependant, les conséquences d'être (par exemple) un 'Turc' ou un 'Néerlandais' étaient moins évidentes et faisaient régulièrement l'objet de discussions. Les élèves ont souvent lié des facteurs tels que la religion, l'apparence, le style ou le comportement à des catégories, bien qu'ils ne soient pas tous du même avis sur la manière dont ces relations s'expriment et sur leur importance au sein d'une certaine catégorie. Cependant, contrairement à l'idée que le fait de se dire 'Marocain', 'Turc' ou 'étranger' est l'expression d'un manque d'intégration ou d'attachement 
émotionnel aux Pays-Bas, les élèves eux-mêmes ont rarement évoqué ces questions lorsqu'ils parlaient de 'Marocains', 'Turcs' ou 'étrangers'. Je voudrais donc souligner dans ce chapitre que l'auto-classification comme 'Marocain', 'Turc' ou 'étranger' ne peut pas être considérée comme un signe de manque de 'sentiment d'appartenance' ou de lien émotionnel avec les Pays-Bas, ni comme un signe de lien avec un autre pays (comme le suggèrent parfois la littérature et le débat social). Chez les élèves de la classe $3 / 4 b$, ces étiquettes ont souvent servi à établir des hiérarchies sociales locales. Il était frappant de constater que des catégories fréquemment stigmatisées dans la société néerlandaise - par exemple les 'Marocains' - avaient un certain prestige parmi les élèves de cette école. Il était 'cool' d'être 'Marocain', 'étranger' ou 'Turc', et la catégorie 'Néerlandais' était associée à des caractéristiques localement indésirables telles que le fait d'être 'sage'. Je suggère dans ce chapitre que ce renversement de la hiérarchie habituelle entre ces catégories (dans laquelle le 'Néerlandais' est souvent considéré comme plus prestigieux) peut être perçu comme une stratégie par laquelle ces élèves atténuent quelque peu leur position marginalisée dans la société néerlandaise. En raison de leur origine étrangère ainsi que de leur position en bas de l'échelle du système éducatif néerlandais (en tant qu'élèves de l'enseignement secondaire professionnel), ils se retrouvent dans au moins deux catégories stigmatisées. En changeant les associations d'une de ces catégories, ces élèves, dans leur contexte local et parmi leurs pairs, ont revendiqué un statut dont ils sont souvent privés en dehors de l'école.

Dans le Chapitre 4, je souligne que les catégories peuvent être considérées non seulement comme une indication de 'qui' est la personne, mais aussi comme des actes en interaction. Grâce à l'analyse de la conversation (CA) et à l'analyse de la catégorisation des membres (ACM), j'analyse ce que les élèves accomplissent dans les interactions en faisant appel aux catégories 'Marocain', 'Turc', 'Néerlandais' et 'étranger'. Étant donné que les catégories dans ce contexte sont liées à toutes sortes de caractéristiques et d'associations (par exemple : 'les Néerlandais et les étrangers ont des coutumes différentes'; ou 'les Marocains sont discriminés'), la mention d'une catégorie évoque une telle association. En utilisant des étiquettes, les élèves pourraient, par exemple, changer de position de pouvoir au sein d'une interaction (par exemple, passer du statut d'élève subordonné' à celui d'expert en coutumes turques'), ou encore taquiner leurs camarades et ainsi désamorcer des interactions potentiellement sensibles ou délicates. L'humour autour des catégories était particulièrement présent chez les élèves de la classe 3/4b. Les élèves issus de l'immigration insultent régulièrement la catégorie dont ils font partie (euxmêmes ou leur interlocuteur) d'une manière qui ressemble fortement aux discours xénophobes répandus. Ces insultes semblaient également servir à ce 


\section{Nederlanders and buitenlanders}

que les élèves s'approprient le discours social xénophobe, le rendant ainsi moins blessant ou menaçant. Mais tous les élèves ne peuvent pas utiliser librement les catégories 'Turc', 'Marocain' ou 'étranger', surtout s'ils sont classés dans la catégorie 'Néerlandais'. Si, par exemple, ils utilisaient les catégories 'Marocain' ou 'Turc' pour taquiner les autres, cela pourrait être considéré comme un écho du discours social. À ces moments-là, il s'est avéré que les catégories ne se limitaient plus à des significations locales et novatrices, mais que leur signification stigmatisante pouvait également réapparaître. Je conclus ce chapitre en constatant que si les catégories peuvent avoir des significations et des fonctions locales, et ne servent pas toujours à déterminer une 'identité', leur utilisation intensive renforce également l'idée que ces différentes catégories représentent une vérité objective.

Dans le Chapitre 5, j'aborde le rôle de la catégorisation dans les interactions avec les enseignants. Ce chapitre montre que dans l'école où j'ai fait mes recherches, les élèves n'étaient pas les seuls à établir des catégories : les enseignants se sont également penchés sur la signification et les fonctions des différentes catégories. Parfois, l'auto-classification des élèves comme étant 'étrangers', 'Turcs' ou 'Marocains' pose problème aux enseignants : ils ont peur que les élèves ne se sentent pas chez eux à l'école ou ils corrigent les élèves en disant qu'ils ne sont 'pas étrangers, mais Néerlandais'. Ceci semble être motivé par la façon dont ces étiquettes sont généralement interprétées, c'est-à-dire que ceux qui se disent non-néerlandais révèlent par là un manque d'intégration ou ne se sentent pas chez eux aux Pays-Bas. Alors que les élèves alternaient entre l'utilisation (parfois sarcastique ou moqueuse) de catégories avec, d'une part, ces associations conventionnelles et, d'autre part, des catégories avec leurs associations locales (comme cela a été principalement analysé dans le Chapitre 3), les enseignants semblaient donc interpréter les catégories principalement de manière conventionnelle. Dans certaines interactions, les réponses des enseignants aux auto-catégorisations des élèves pourraient également être perçues comme un acte en interaction, tout comme les catégorisations des élèves eux-mêmes. En ce sens, la contestation d'une auto-classification comme 'étranger' pourrait également servir à souligner l'autorité, à rétablir l'ordre ou à discipliner un élève. Bien que les enseignants se soient montrés favorables à ce que leurs élèves se classent tous dans la catégorie des 'Néerlandais', ils ont, à d'autres occasions, fait une nette distinction entre les élèves issus de l'immigration et ceux qui n'en sont pas. Ils semblent avoir du mal à concilier leur désir de voir leurs élèves se considérer comme 'Néerlandais' et leur conviction qu'il existe de nettes différences entre les élèves avec et sans origine étrangère - des différences qui sont d'ailleurs soulignées par les élèves euxmêmes. Je conclus ce chapitre en soulignant qu'il n'est pas nécessairement mauvais ou irresponsable pour les enseignants d'utiliser les mêmes étiquettes 
que leurs élèves. Certains enseignants ont utilisé des étiquettes d'une manière très similaire à celle dont les élèves les utiliseraient, par exemple en taquinant, et cela peut en fait contribuer à améliorer la relation élève-enseignant. Cependant, si les enseignants souhaitent promouvoir l'inclusion dans ce contexte, il semble peu probable qu'ils puissent y parvenir en contestant les auto-catégorisations telles que 'Marocain', 'Turc' ou 'étranger', ou en encourageant les élèves à s'appeler 'Néerlandais'. Notamment parce que l'étiquette 'Néerlandais' a une signification indésirable pour les élèves ; d'une part, parce qu'ils sont souvent considérés comme non Néerlandais par les 'Néerlandais' et, d'autre part, parce que cette catégorie est également associée ici, dans ce contexte local, à des connotations non cool et 'trop scolaires'. La promotion d'un sentiment d'inclusion pourrait par conséquent être améliorée en utilisant d'autres catégories ne présentant pas cette variété de significations locales et conventionnelles complexes d'une étiquette telle que 'Néerlandais'.

Si l'organisation symbolique des catégories décrite dans les chapitres 3 à 5 est l'un des moyens par lesquels les élèves se confrontent aux hiérarchies sociales locales et plus générales, le Chapitre 6 décrit comment le multilinguisme et l'organisation symbolique des ressources linguistiques en est un autre. Si l'organisation symbolique des catégories décrite dans les chapitres 3 à 5 est l'un des moyens par lesquels les élèves se confrontent aux hiérarchies sociales locales et plus générales, le Chapitre 6 décrit comment le multilinguisme et l'organisation symbolique des ressources linguistiques en est un autre. Bien que l'école de Venlo n'ait pas de politique linguistique explicite, les enseignants considéraient comme évident que tout le monde devait parler le néerlandais standard en classe. Cela a provoqué des tensions car les élèves avaient des compétences linguistiques très variées : outre le néerlandais standard (qui est la langue la plus utilisée par les enseignants et les élèves dans leur communication à l'école), certains élèves parlaient par exemple le limbourgeois, l'arabe marocain, le berbère ou le turc, et se servaient de ces langues dans leurs interactions quotidiennes à l'école. La signification de l'utilisation de ces ressources linguistiques varie : parmi les élèves, et à l'école, le recours à des expressions en arabe marocain, en turc ou en berbère est considéré comme prestigieux, contrairement au fait de parler le limbourgeois. Ainsi, chez les élèves, l'organisation symbolique des langues reflète les schémas de hiérarchisation des catégories chez les élèves tels qu'ils sont explorés dans les Chapitres 3 à 5. L'organisation symbolique des mêmes langues a suivi un schéma opposé en ce qui concerne les expressions des enseignants. Ils ont appliqué la politique de la langue néerlandaise standard, en particulier lorsque les élèves parlaient l'arabe, le berbère ou le turc, et non pas lorsque les élèves parlaient le dialecte de Venlo ou de Tegelen. Ils le justifiaient en se référant aux idéologies des langues et des locuteurs associés à des endroits spécifiques. 
Le limbourgeois a été jugé acceptable parce qu'après tout, l'école était située dans le Limbourg, alors que le turc, par exemple, était considéré comme une langue étrangère et donc inappropriée dans le contexte donné. Dans ce chapitre, j'ai souligné que l'accent mis par les enseignants sur le néerlandais standard lorsque les élèves parlaient turc, arabe ou berbère, et l'absence d'interférence lorsque les élèves parlaient limbourgeois, indiquent que les préoccupations relatives à l'utilisation de la langue aux Pays-Bas ont changé et évoluent. Alors que l'utilisation du dialecte dans le Limbourg était autrefois considérée comme 'non civilisée' et associée à un désavantage (éducatif), cette stigmatisation semble avoir largement disparu, du moins dans ce contexte (bien que les dialectes soient encore principalement utilisés dans la sphère informelle). Les préoccupations concernant la langue en tant que signe de 'civilisation' ont évolué vers des préoccupations concernant la langue en tant que symbole d'appartenance'. Les inquiétudes des enseignants quant au fait que leurs élèves se sentent ou non 'chez eux' ont été reconnues dans leurs réactions aux auto-classifications de leurs élèves, ainsi que dans leurs réactions face à l'utilisation d'autres langues que le néerlandais. Cela montre comment l'organisation symbolique des langues reproduit des préoccupations sociales et des modèles de stratification sociale en constante évolution.

\section{Contributions scientifiques et sociétales}

Je voudrais souligner quatre contributions de cette thèse à la science et à la société. Tout d'abord, j'espère avoir démontré que les significations et les fonctions des catégories ethniques ne peuvent être considérées comme acquises ou évidentes, mais doivent être étudiées dans leur contexte. J'ai contribué à une compréhension plus approfondie de la catégorisation ethnique en analysant ses significations et ses fonctions interactionnelles locales, en tenant compte de la manière dont ces significations et fonctions locales sont en lien avec les discours qui se produisent à plus grande échelle sociale aux Pays-Bas. Dans plusieurs pays ayant une histoire d'immigration similaire, on a observé que les étrangers et leur descendance se catégorisent en fonction de leur pays d'origine plutôt que de leur pays de résidence (par exemple en Allemagne, voir Bozay 2012, en France, voir Simon 2012, et en Belgique, voir Jaspers 2011a). Bien que de nombreux travaux sociolinguistiques et anthropologiques aient insisté sur le fait que les identités ethniques sont spécifiques au contexte, intersubjectives et discursives, et qu'elles se négocient en interaction (voir par exemple Androutsopoulos et Georgakopoulou 2003 ; Jaspers 2005b ; Jørgensen 2005 ; Rampton 2005), l'auto-catégorisation par pays d'origine est encore souvent interprétée comme un manque d'identification avec le pays de résidence (voir par exemple Agirdag, van 
Houtte et van Avermaet 2011 ; Ersanilli et Koopmans 2010 ; Huijnk et Dagevos 2012 ; Phalet et Swyngedouw 2002 ; Verkuyten et Yildiz 2007).

Un message important de cette thèse est que, pour savoir ce que signifient les étiquettes ethniques et les raisons pour lesquelles les gens les utilisent, il est nécessaire d'étudier les données interactionnelles dans un contexte que le chercheur connaît bien ; en d'autres termes, il faut adopter une approche sociolinguistique et ethnographique dans laquelle les conditions locales et les processus à plus grande échelle sont pris en considération. Cette démarche m'a permis de montrer que l'auto-classification avec les étiquettes 'Marocain', 'Turc' ou 'étranger' n'indique pas nécessairement un manque de 'sentiment d'appartenance' ou de lien avec les Pays-Bas, ni la présence d'un attachement à un autre pays. Les participants à cette étude ont utilisé les catégories, entre autres, pour établir des hiérarchies sociales locales. De plus, cette thèse a démontré qu'il est possible de dépasser le concept d'identité' dans l'analyse de la catégorisation en termes ethniques. Même si l'on utilise des termes 'ethniques', les locuteurs ne font pas simplement référence à leur identité ethnique. Les participants à cette recherche se sont aussi servis d'étiquettes comme moyen d'action : en contredisant les autres, en déplaçant les positions de pouvoir des institutions ou en se taquinant les uns les autres. En mettant l'accent sur les fonctions interactionnelles des catégories ethniques, $j$ 'espère lutter contre une 'fétichisation de l'ethnicité' (Rampton 2005), c'est-à-dire la tendance à étiqueter les individus comme 'exotiques' et 'différents' et à percevoir 1'identité ethnique' dans les actions quotidiennes.

En second lieu, j'ai montré que la combinaison de méthodes ethnographiques pour le recueil de données avec des méthodes ethnométhodologiques pour l'analyse de ces données permettait d'éviter à la fois un constructivisme réduit à la platitude et la tendance aux généralisations que Brubaker (2002, 2004) en particulier souligne dans l'étude de l'identification ethnique. Brubaker constate que de nombreux chercheurs déclarent adopter une approche 'constructive' de l'ethnicité, mais qu'en même temps, ils partent souvent du principe de la présence de 'groupes ethniques' au sein de leurs analyses. Mon but dans cette thèse était d'éviter de telles généralisations en traitant les catégories ethniques comme un objet d'analyse plutôt que comme un instrument d'analyse, en expliquant la notion obstinée d'ethnicité dans la vie des participants sans pour autant présenter l'ethnicité comme une vérité objective. J'ai fait cela en combinant l'ethnographie avec l'analyse de la conversation (CA) et l'analyse de la catégorisation des membres (ACM).

D'une part, la base ethnographique de la thèse renforce les analyses des interactions, en partie parce que la plupart des interactions que j'analyse sont retenues comme exemples d'interactions habituelles entre les participants. 
Sans l'implication à long terme du travail ethnographique de terrain, je n'aurais pas été en mesure de distinguer les interactions habituelles et inhabituelles, ni d'argumenter les fonctions interactionnelles que les différentes catégorisations avaient dans ce contexte. D'autre part, l'ethnographie m'a aidé à explorer le lieu des interactions et à reconnaître que chaque langue a son propre contexte historique, social et culturel. L'accent qui est mis sur la réflexivité en ethnographie m'a également incité à explorer l'influence de ma présence sur le contexte et les interactions que j'analyse. L'utilisation de l'AC et de l'AMC a renforcé le côté ethnographique de cette thèse grâce aux méthodes d'analyse caractéristiques, systématiques et approfondies que ces dernières requièrent. La transcription détaillée de CA et MCA a permis d'examiner mes données et mes arguments avec d'autres chercheurs, ce qui permet à d'autres personnes d'examiner ces données et de contester mes analyses. Par-dessus tout, l'utilisation de l'AC et de l'AMC m'a encouragé à me concentrer sur les actes de mes participants dans les interactions, ce qui m'a permis de voir au-delà des étiquettes 'ethniques'.

La troisième contribution de cette thèse réside dans le fait qu'elle a démontré la nécessité d'étudier les manifestations de la 'diversité' (notamment en termes d'ethnicité et de langue) résultant de la mondialisation dans des lieux considérés comme périphériques. De nombreuses recherches sur les effets de la mondialisation se sont concentrées sur les endroits où les conséquences en sont clairement visibles, en particulier dans les grandes villes où la diversité des personnes et des langues est importante (Wang et al. 2014). Cornips et de Rooij (2018b) argumentent qu'un tel focus ne se justifie pas car la mondialisation se manifeste aussi dans ce qui est considéré comme des zones périphériques. Cette thèse contribue à corriger cette tendance en étudiant la manifestation discursive de la mondialisation (l'utilisation d'étiquettes ethniques) dans la ville de Venlo, considérée comme étant une ville périphérique au niveau national. $28,4 \%$ des habitants de Venlo ont une origine étrangère, contre 23,6 \% aux Pays-Bas (Centraal Bureau voor de Statistiek 2019a). Dans la classe 3/4b, environ la moitié des élèves étaient issus de l'immigration et ils faisaient quotidiennement référence à ce contexte. Les signes de la mondialisation étaient donc indéniables dans le cadre de cette étude. La manière dont elle s'est traduite dans l'auto-catégorisation des élèves a néanmoins été influencée par cet environnement spécifique. Alors que dans des villes comme Amsterdam et Rotterdam, il a été constaté que de nombreuses personnes issues de l'immigration s'appellent 'Amsterdammer' ou 'Rotterdammer' au lieu de 'Néerlandais' (Omlo 2011; van der Welle 2011; Özpamuk 2018), je n'ai jamais constaté que les élèves de la classe 3/4b s'appelaient 'Venloaar' ou 'Limburger'. Cela pourrait être dû aux caractéristiques spécifiques de Venlo et du Limbourg. Le fait que les gens 
associent souvent le Limbourg à une forte identité locale et à des caractéristiques socioculturelles et linguistiques spécifiques pourrait rendre les étiquettes 'Limburger' ou 'Venlonaar' moins accessibles à ceux qui sont généralement considérés comme 'divers'.

$\mathrm{La}$ quatrième contribution de cette thèse est que, alors que de nombreuses études se concentrent soit sur l'utilisation des 'langues des immigrants', soit sur l'utilisation du dialecte en classe, j'ai décidé de comparer les deux, dans une certaine mesure, au sein de cette thèse. Ce faisant, j'ai analysé l'organisation symbolique des langues et de leurs locuteurs dans la classe $3 / 4 b$, et démontré comment cette organisation symbolique reflète et reproduit des schémas plus larges de stratification sociale. La comparaison des dialectes et des 'langues des immigrants' a révélé que si le limbourgeois est toujours considéré comme acceptable, principalement dans la sphère informelle, et n'a donc pas de place officielle dans l'enseignement, il n'est plus aussi stigmatisé qu'auparavant. Les préoccupations concernant l'utilisation de la langue comme signe de 'civilisation', qui étaient à la base de la stigmatisation du limbourgeois, se sont transformées en préoccupations concernant la langue et l'appartenance'. À la base de cette approche se trouvent des idéologies linguistiques telles que l'idée qu'il existe des liens inextricables entre les personnes, les langues et les lieux, ce qui fait que certaines langues et leurs locuteurs sont considérés comme 'appartenant' ou non à la communauté (Thissen 2018). Dans un climat sociopolitique où les personnes issues de l'immigration sont confrontées à de nombreux défis, ce sont les langues associées à ces mêmes personnes qui subissent aujourd'hui les appréhensions et les critiques que les dialectes ont connues par le passé. Les tendances en matière d'application de la politique de la langue néerlandaise standard à l'école - où, en général, les enseignants mettaient en œuvre la politique de manière beaucoup plus stricte lorsque les élèves parlaient turc, arabe ou berbère que lorsqu'ils parlaient un dialecte - sont le reflet des préoccupations sociales actuelles aux Pays-Bas, et en particulier de la question posée dans de nombreux débats sociaux et politiques, à savoir dans quelle mesure les personnes issues de l'immigration se sentent (suffisamment) chez elles aux Pays-Bas (Duyvendak, Reinders et Wekker 2016). 
244 Nederlanders and buitenlanders 


\section{Valorization addendum}

'Wanneer ben je écht Nederlander?,' vraagt Olaf Tempelman zich af in de Volkskrant van 7 februari 2020. ${ }^{37}$ In zijn artikel beschrijft hij een man die na twintig jaar in Nederland gewoond te hebben naar Canada is geëmigreerd. "Ik ben hier pas zes maanden en voor al mijn klanten ben ik nu al een Canadees. Voor mijn Nederlandse klanten was ik na twintig jaar nog steeds geen Nederlander, niet eens een Poolse Nederlander, ik bleef een Pool," aldus de man in het artikel van Tempelman. Ook in een artikel in de Limburger van 10 mei $2018^{38}$ wordt aandacht besteed aan wat 'etiketjes' worden genoemd: de geïnterviewde wordt geïntroduceerd als Marokkaan, Nederlander, Marokkaanse Nederlander, Venlonaar, Marokkaanse Venlonaar, Arabische Marokkaan, Limburger, en Europeaan. Beide artikelen illustreren hoezeer het gebruikelijk is in Nederland om elkaar te categoriseren naar afkomst en/of etniciteit, en in het geval van het eerste artikel, hoe uitsluitend het gebruik van zulke labels kan werken. In dit proefschrift heb ik het gebruik van dit soort etnische labels van verschillende kanten belicht. Juist omdat dit type categorisering in de samenleving zo gangbaar is, zijn de resultaten van dit onderzoek niet alleen van belang voor de wetenschap, maar ook voor de samenleving in zijn geheel. Tijdens mijn promotietraject heb ik dan ook getracht om via verschillende wegen de brug van wetenschap naar samenleving te slaan. In dit addendum zet ik ten eerste uiteen welke activiteiten ik daartoe heb ondernomen gedurende het promotie-traject, en vervolgens beschrijf ik verdere kansen voor kennisvalorisatie in de toekomst.

\section{Kennisvalorisatie tijdens het onderzoek}

In juni $2017 \mathrm{kwam} \mathrm{ik}$, naar aanleiding van een presentatie over mijn onderzoek tijdens een conferentie over immigratie in Limburg bij het CBS in Heerlen, ${ }^{39}$ in aanraking met een gemeenteraadslid van de gemeente Venlo. De gemeente was op dat moment bezig met een onderzoek naar de lage representatie op de

${ }^{37} \mathrm{Zie}$ https://www.volkskrant.nl/cs-b631ab63 (laatst bezocht op 14 mei 2020).

${ }^{38} \mathrm{Zie}$ https://www.limburger.n1/cnt/dmf20180509 00061471/de-vele-etiketjesvan-marokkaanse-venlonaar-zakaria-bouders (laatst bezocht op 14 mei 2020).

${ }^{39}$ van de Weerd, Pomme. "Identificatiepraktijken onder VMBO'ers in Limburg" (presented at Small Data, Big Data en Immigranten in Limburg, CBS, Heerlen, June 1, 2017). 
Venlose arbeidsmarkt van mensen met een zogenaamde niet-westerse migratieachtergrond in vergelijking met veel andere Nederlandse steden (gebleken uit Atlas voor gemeenten 2014). Om de oorzaken hiervan te achterhalen organiseerde de gemeenteraad in samenwerking met verschillende organisaties themabijeenkomsten over enkele onderwerpen die hieraan ten grondslag zouden kunnen liggen, en over hoe dit probleem mogelijk aan te pakken zou kunnen zijn. In november 2017 werd ik uitgenodigd om een presentatie te geven over mijn promotieonderzoek tijdens een raadsledenvergadering, in voorbereiding op de themabijeenkomst over ' $\mathrm{T}$ aal en Cultuur.' Mijn doel in de presentatie was om te benadrukken dat taal en cultuur niet onveranderlijke en statische objecten zijn die zich bij iedereen van een bepaalde afkomst op één manier manifesteren. Ik beschreef hoe ik tijdens mijn onderzoek, waarvoor ik op dat op dat moment al een aantal maanden mee had gelopen met een schoolklas in een Venlose middelbare school, had opgemerkt dat alle leerlingen, ongeacht afkomst, zich wel eens in Limburgs uitdrukten; dat ook alle leerlingen, ongeacht afkomst, zich wel eens bedienden van uitdrukkingen in het Arabisch, Berbers, of Turks; en dat leerlingen zichzelf regelmatig labelden als 'Marokkaan' of 'Turk,' maar dat dat niet voor iedere leerling dezelfde betekenis had. Ik benadrukte op basis van die observaties dat zo'n label niet begrepen kan worden als direct verbonden aan het 'hebben' van een cultuur, of het gebruiken van een bepaalde taal. Hiermee trachtte ik de gemeenteraad uit te dagen om verder te denken dan vaste categorieën mensen die, door hun veronderstelde lidmaatschap in een toegewezen categorie als 'iemand met niet-Westerse migratieachtergrond,' bepaalde talen wel of niet (voldoende) spreken of bepaalde culturele karakteristieken bezitten. Ik hoopte te laten zien dat de situatie, zoals ik die geobserveerd had in de context van de middelbare school, anders in elkaar zat en genuanceerdere observaties behoefde.

$\mathrm{Na}$ deze presentatie en het gesprek dat daarop volgde heb ik de daaraan gerelateerde thema-bijeenkomst in de moskee in Venlo bijgewoond, georganiseerd door de gemeenteraad, waarbij verschillende belanghebbenden (ouders, docenten, gemeenteraad) aanwezig waren. Hier werd in een grotere kring gesproken over 'taal' en 'cultuur' als mogelijke factoren in de suboptimale positie van mensen met een migratieachtergrond op de arbeidsmarkt in Venlo. Hier heb ik geprobeerd bij te dragen met huidige visies op, en onderzoeksresultaten over, de ontwikkeling van meertaligheid. Bovendien heb ik meegepraat over het concept 'cultuur' en hoe dat productief te begrijpen is in een toegepaste context en in betrekking tot een concreet probleem (dat van werkloosheid). Tot slot ben ik betrokken geweest bij de eindrapportage over het onderzoek van de gemeente. Hierin heb ik aan aantal bijdragen geleverd, waaronder de aanbeveling om de term 'allochtoon' uit het rapport te vervangen 
voor 'persoon met een migratie-achtergrond' (in navolging van wat inmiddels de standaard was geworden bij bijvoorbeeld de overheid en het Centraal Bureau voor de Statistiek), en heb ik verdere relevante literatuur aangedragen.

Met het doel om ook docenten te bereiken met een aantal van mijn bevindingen over zelf-categorisering van jongeren als 'Marokkaan,' 'buitenlander' of 'Nederlander,' schreef ik in 2018 een artikel voor het vakblad van de Internationale Vereniging voor de Neerlandistiek, VakTaal, met als titel: 'Leerlingen en labels: Wie zijn dan die buitenlanders?' ${ }^{30}$ Dit blad wordt voornamelijk gelezen door docenten Nederlands in het middelbaar onderwijs. In dit artikel schrijf ik over mijn veldwerk op de middelbare school in Venlo. Ik beschrijf hoe ik de leerlingen daar leerde kennen, en dat ik me verbaasde toen ik hoorde dat leerlingen die geboren en getogen waren in Nederland zichzelf stellig 'buitenlander' noemden. Verder leg ik uit hoe ik van mijn initiële verbazing en onbegrip kwam tot het inzicht dat een 'buitenlander' (net als een 'Marokkaan,' een 'Turk,' en een 'Nederlander') voor hen heel andere associaties had dan voor mij. Voor hen zeiden die labels niet per definitie iets over of ze zich wel of niet thuis voelden in Nederland, en hadden ze ook geen negatieve, maar juist positieve connotaties bij deze labels. Tenslotte benadrukte ik dat de connotaties, en de gevolgen van het gebruiken van zo'n label, wel afhankelijk is van wie ze gebruikt, omdat de leerlingen zich duidelijk ook bewust waren van potentiële negatieve toepassingen.

Tijdens een openbare lezing in Maastricht in het kader van de Dag van de Moedertaal op 21 februari 2018 ging ik in op het fenomeen 'jongerentaal. ${ }^{41}$ Vaak wordt er met zorg gekeken (of, beter gezegd, geluisterd) naar de manieren waarop jongeren praten: er wordt gezegd dat ze geen 'correct' Nederlands kunnen spreken, en dat dit hen in de weg zit, bijvoorbeeld bij het zoeken naar een baan. In de presentatie liet ik een fragment muziek horen waarin uitdrukkingen uit allerlei andere talen werden gebruikt in een verder Nederlandse tekst - een kenmerk van zogenaamde 'jongerentaal.' Ik besprak de herkomst van allerlei uitdrukkingen uit dit fragment, en beschreef hoe het proces van indexicalisatie ten grondslag licht aan dit type taalgebruik. Het concept indexicalisatie beschrijft hoe talige uitdrukkingen bepaalde associaties en waardes krijgen, en kan worden gebruikt om uit te leggen hoe en waarom bepaalde taal zo populair wordt onder jongeren. $Z_{o}$ beargumenteerde ik dat

${ }^{40}$ van de Weerd, Pomme. 2018. Leerlingen en labels: Wie zijn dan die buitenlanders? VakTaal 31(3): 20-21.

${ }^{41}$ van de Weerd, Pomme. 2018. "Jongerentaal door de ogen van een taalkundig antropoloog: Verloedering of kapot creatief?" (presented at Dag van de Moedertaal, Maastricht, February 21, 2018). 


\section{Nederlanders and buitenlanders}

dit type taalgebruik niet voortkomt uit gebrekkige kennis van Nederlands, maar dat het juist een heel creatieve talige uiting is waarmee jongeren zich kunnen identificeren met bepaalde sociale groepen.

In 2017, 2018 en 2019 heb ik gastcolleges verzorgd over het onderwerp 'Taal, cultuur, en identiteit' voor studenten van de minor Meertaligheid bij de studie logopedie van Hogeschool Rotterdam. Tijdens deze gastcolleges besprak ik associaties tussen taal en identiteit met studenten, onder andere hoe bepaalde manieren van spreken verbonden worden met stereotypes van personen. Vervolgens besprak ik steeds andere elementen van mijn eigen onderzoek. Vaak gaf ik de studenten een transcript van een interactie onder de leerlingen tijdens mijn veldwerk, en vroeg hen om dit te analyseren. Op die manier leerden zij tijdens deze colleges niet alleen van mij, maar ging de kennisoverdracht beide kanten op: ik leerde ook veel van hun interpretaties van de uitspraken van de leerlingen in Venlo. In 2017 gaf ik een soortgelijk gastcollege voor studenten van de Hogeschool Zuyd in Maastricht.

In het kader van valorisatie alsmede reciprociteit verzorgde ik in december 2018 een presentatie op de school waar ik mijn onderzoek heb uitgevoerd. Tijdens de wekelijkse docentenvergadering vertelde ik wat mijn belangrijkste resultaten waren, me hierbij concentrerend op de uiteenlopende en vaak onverwachte betekenissen voor leerlingen van de labels 'Marokkaan,' 'Turk,' en 'buitenlander.' Een aantal docenten gaf aan hierdoor bevestigd te zijn in wat zij al dachten, maar er waren ook docenten die zeiden dat mijn presentatie voor hen een 'eye-opener' was en dat zij hun leerlingen nu beter dachten te begrijpen.

\section{Verdere kansen voor kennisvalorisatie}

Zoals ik schreef in de opening van dit valorisatie addendum, is het in Nederland (net als in veel andere landen) gebruikelijk om mensen te categoriseren naar aanleiding van hun afkomst. Een aantal jaar geleden woedde de discussie over het woord 'allochtoon,' en besloot de overheid, net als veel kranten en organisaties, dit woord te vervangen door 'persoon met een migratie-achtergrond.' Desondanks wordt er alsnog regelmatig gepraat en geschreven over 'Marokkanen' of 'Turken' wanneer het gaat om mensen wiens ouders of grootouders uit Marokko of Turkije kwamen, en worden mensen met een migratieachtergrond regelmatig gevraagd 'waar ze vandaan komen' (waarbij gedoeld wordt op een plaats buiten Nederland). Tegelijkertijd wordt er vaak met verbazing of onbegrip gereageerd als iemand zichzelf 'Marokkaan' of 'Turk' noemt in plaats van 'Nederlander.' Mensen met een migratieachtergrond zitten daarmee gevangen in een patstelling waarbij zij enerzijds herhaaldelijk buiten een concept van 'Nederlanderschap' geplaatst, 
en tegelijkertijd erop worden aangekeken als zij zichzelf niet als 'Nederlander' definiëren. Ik hoop dat de bevindingen uit mijn proefschrift kunnen helpen om meer bewustzijn te creëren over het gebruik van zulke etnische labels. Ten eerste hoop ik dat ik, bijvoorbeeld door middel van populaire publicaties in media of openbare presentaties, kan benadrukken dat zelf-categorisering in etnische termen begrepen moet worden in de context van gebruik, en dat labels als Marokkaan of Turk dus geen eenduidige betekenissen hebben. Daarbij hoop ik, ten tweede, dat dat niet gepaard gaat met het idee dat iedereen die labels in elke context kan gaan gebruiken. Het gaat in de kwestie van labelen niet slechts om wat er wordt gezegd (ofwel welk label er wordt gebruikt), maar ook hoe, wanneer, en met name wie dat label gebruikt.

Groter bewustzijn hiervan zou van algemeen belang kunnen zijn voor iedereen in Nederland. We zijn allemaal gebaat bij het beter begrijpen van wie wij als de 'ander' zien, of wie zich als 'anders' aan ons presenteert. De meesten van ons komen op enig moment van ons leven, direct of indirect, in contact met mensen die zich identificeren als iets anders, of naast, 'Nederlander.' Aandacht voor, en enige kennis van, de genuanceerde, variërende, en complexe betekenissen van zulke labels - en veel andere labels, bijvoorbeeld rondom regionaliteit (bijv. 'Limburger'), gender, seksualiteit, religie, huidskleur, et cetera - zou kunnen helpen om minder snel oordelen te vellen en open te staan voor de ander. Hoewel het dus in mijn mening voor iedereen verrijkend zou kunnen zijn om kennis te nemen van bepaalde conclusies van mijn onderzoek, zijn er enkele doelgroepen die, in mijn ogen, hier direct baat bij zouden kunnen hebben.

Ten eerste zou het goed zijn om deze bevindingen te circuleren onder professionals in de media. Een belangrijke bron van associaties met labels is kranten die schrijven over 'niet Westerse leerlingen,' of tv-programma's waarin het woord 'buitenlander' wordt gebruikt voor mensen die in Nederland geboren en getogen zijn. Hetzelfde geldt voor het gebruik van labels als 'Limburger.' Deze woorden zijn niet neutraal, maar zijn als een soort container waar allerlei associaties in komen te zitten. Iedere keer dat iemand dit woord dan gebruikt, worden die associaties opgeroepen. Het is daarom belangrijk dat journalisten hier bewust van worden, en stereotyperende categorisering vermijden.

Een andere kans voor kennisvalorisatie zou zijn om workshops te organiseren op middelbare en hogere scholen waarbij een dialoog wordt gestimuleerd tussen docenten en leerlingen over het gebruik en de betekenissen van categorieën als buitenlander. Het doel hiervan zou zijn om de docenten en leerlingen samen te laten reflecteren over wat labels als Marokkaan of Turk voor hen betekenen en te ontdekken dat er daar misschien verschillen tussen bestaan. Verder zouden leerlingen en docenten kunnen 
leren over kwesties als identiteit, cultuur, taal, en categorieën, en zouden ze aan den lijve kunnen ondervinden dat de relaties tussen die zaken niet eenduidig zijn, maar juist flexibel en vaak onverwacht. Dit zou kunnen leiden tot beter begrip tussen docenten en leerlingen, maar ook tussen docenten zelf, en tussen leerlingen zelf. Zo'n workshop zou deel kunnen uitmaken van vakken als maatschappijleer of burgerschap. 


\begin{abstract}
About the author
Pomme van de Weerd was born in Apeldoorn, the Netherlands, on July 28th, 1990. She obtained her bachelor's degree in Liberal Arts and Sciences (Cum Laude) at University College Utrecht, with a major in humanities (linguistics) and a minor in anthropology. In the following two years, she was part of a research team in Santiago de Chile conducting research on contemporary uses of the language Mapudungún, and taught English in local companies. In 2016, she graduated from the Research Master program Cultural Anthropology: Sociocultural Transformation at Utrecht University, having conducted ethnographic fieldwork in Santiago de Chile on the sociocultural impact of upward social mobility. Directly after her Masters, Pomme started her $\mathrm{PhD}$ at Maastricht University on multilingualism and identity among pupils of a vocational track in Limburg. She has published in international peer-reviewed journals such as Journal of Sociolinguistics and has presented her work at international conferences. During her $\mathrm{PhD}$, Pomme worked as a lecturer for the master's program Dutch as a Second Language and Multilingualism at the University of Amsterdam, as well as in the bachelor programs Dutch Studies and Linguistics, and she spent three months as a visiting scholar at University of California, Santa Barbara. Currently, she is employed as a lecturer at the University of Amsterdam and Amsterdam University College.
\end{abstract}

